Aus der Abteilung Kieferorthopädie

(Prof. Dr. med. dent. D. Kubein-Meesenburg)

im Zentrum Zahn-, Mund- und Kieferheilkunde

der Medizinischen Fakultät der Universität Göttingen

\title{
Die biomechanische Funktion der Articulationes zygapophysiales der Brustwirbelsäule
}

\section{Eine 6-D-Analyse der Bewegungsstrukturen des Segments Th2 / Th3}

\author{
Inaugural - Dissertation \\ zur Erlangung des Doktorgrades \\ für Zahnheilkunde der Medizinischen Fakultät \\ der Georg-August-Universität zu Göttingen
}

\author{
vorgelegt von \\ Julia Saptschak \\ aus Aktjubinsk / Kasachstan
}

Göttingen 2009 
Dekan: Prof. Dr. med. C. Frömmel

I. Berichterstatter: Prof. Dr. rer. nat. H. Nägerl

II. Berichterstatter: Priv.- Doz. Dr. med. Dumont

III. Berichterstatter/in:

Tag der mündlichen Prüfung: 24.06.2010 


\section{Inhaltsverzeichnis}

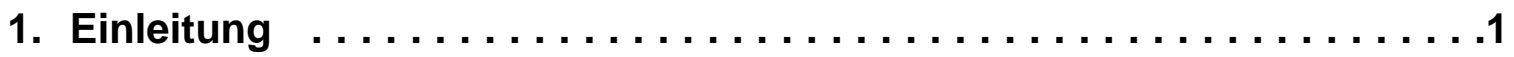

1.1 Einführung $\ldots \ldots \ldots \ldots \ldots \ldots \ldots \ldots \ldots \ldots \ldots \ldots \ldots$

1.2 Anatomie der Wirbelsäule $\ldots \ldots \ldots \ldots \ldots \ldots \ldots \ldots \ldots, 8$

1.3 Mathematische und physikalische Grundlagen ... . . . . . . . 20

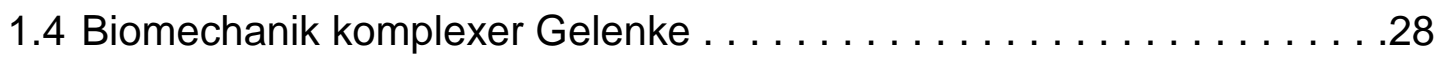

2. Material und Methoden $\ldots \ldots \ldots \ldots \ldots \ldots \ldots \ldots \ldots \ldots \ldots \ldots . .44$

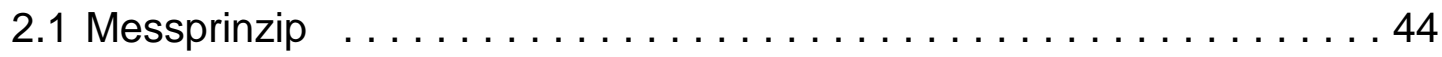

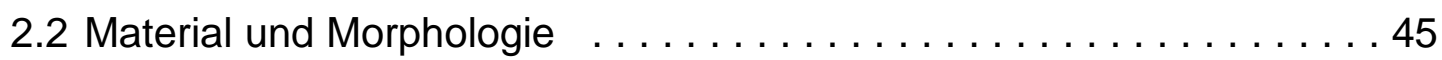

2.3 Messapparatur. ........................... 50

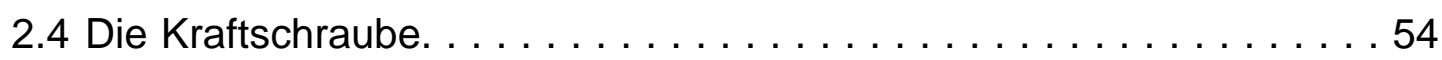

2.5 Die Applikation von Drehmoment. . . . . . . . . . . . . . . 57

2.6 Analyse der Positionsveränderungen $\ldots \ldots \ldots \ldots \ldots \ldots \ldots \ldots . . .62$

2.7 Präsentation der relevanten Verschraubungsparameter . . . . . . . 67

2.8 Ablauf der Messung . . . . . . . . . . . . . . . . . . ... 68

3. Ergebnisse $\ldots \ldots \ldots \ldots \ldots \ldots \ldots \ldots \ldots \ldots \ldots \ldots \ldots \ldots$

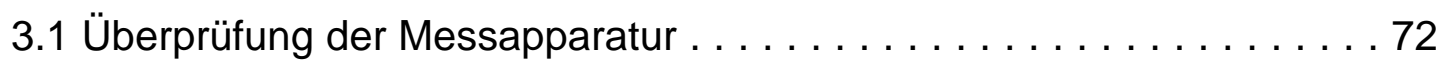

3.2 Axiale Rotation . . . . . . . . . . . . . . . . . . 78

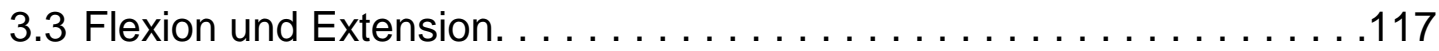

3.4 Lateralflexion . . . . . . . . . . . . . . . . . . . . . . 139

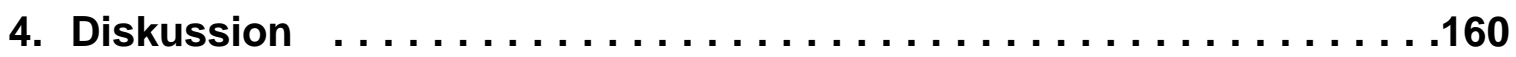

4.1 Die Bewegungsstruktur eines Brustwirbelsegments Th2/3 . . . . . 160

4.2 Methodendiskussion . . . . . . . . . . . . . . . . . . . . . . . 172

4.3 Messsystem . . . . . . . . . . . . . . . . . . 174

4.4 Wahl der Präparate $\ldots \ldots \ldots \ldots \ldots \ldots \ldots \ldots \ldots \ldots$ 
4.5 Ausblick ................................... 176

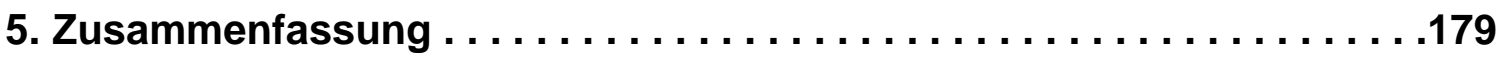

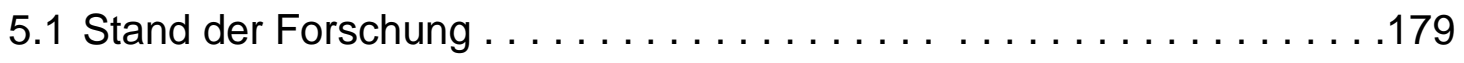

5.2 Experimentelle Methode. . . . . . . . . . . . . . . . . . . . . . 179

5.3 Ergebnisse. . . . . . . . . . . . . . . . . . . . . 181

5.4 Diskussion. . . . . . . . . . . . . . . . . . . . . . . .

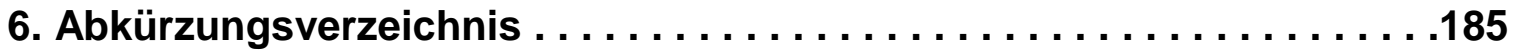

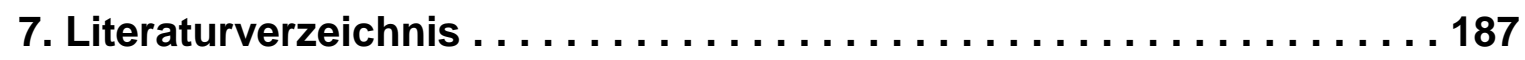




\section{Abbildungs- und Tabellenverzeichnis}

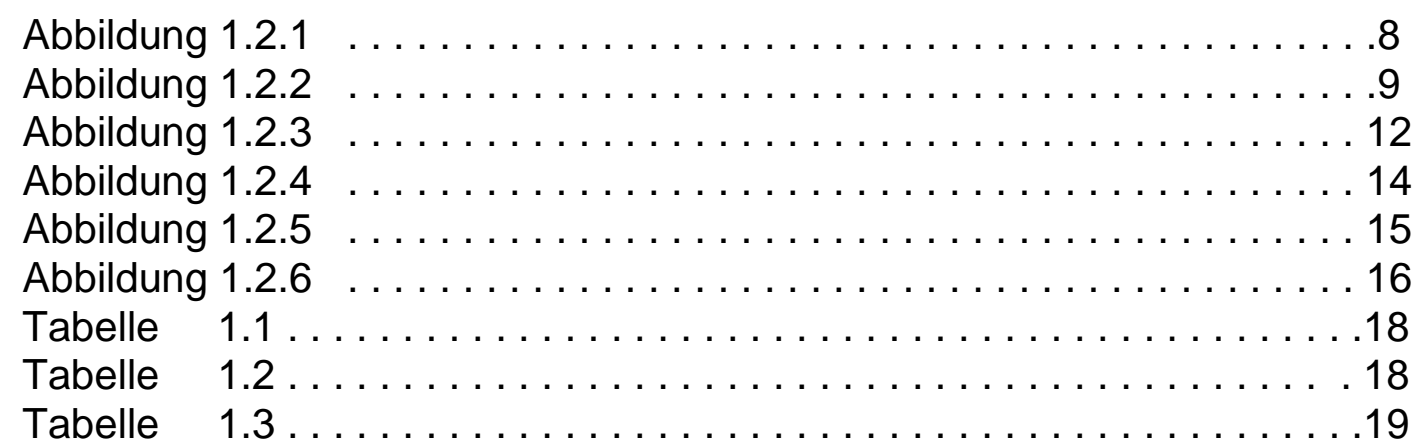

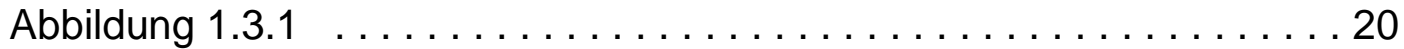

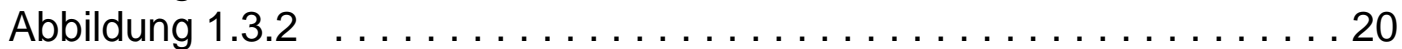

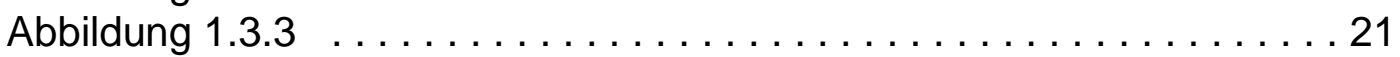

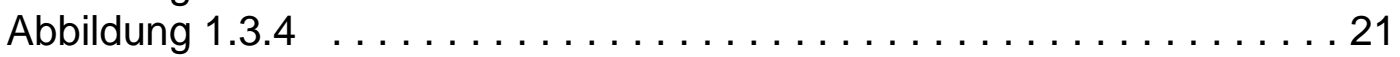

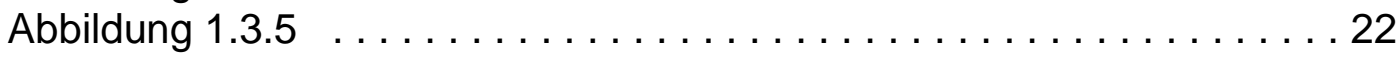

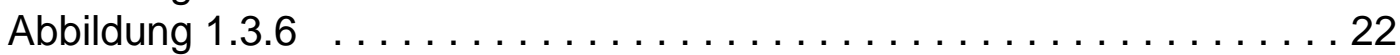

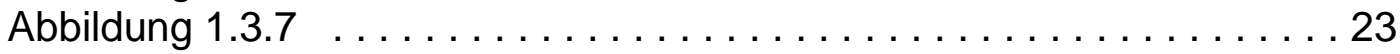

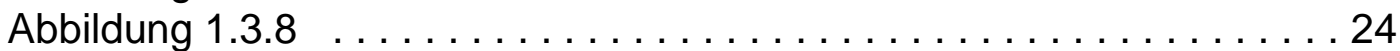

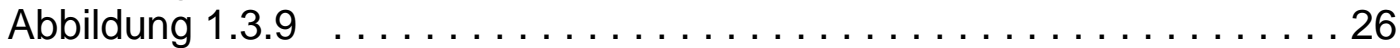

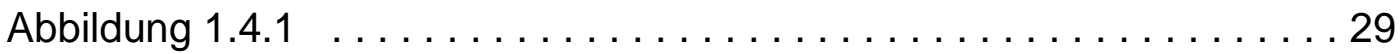

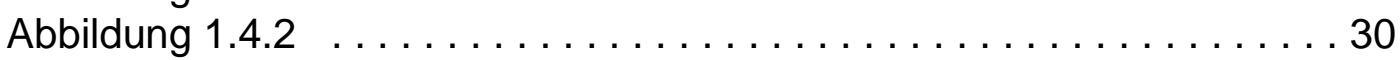

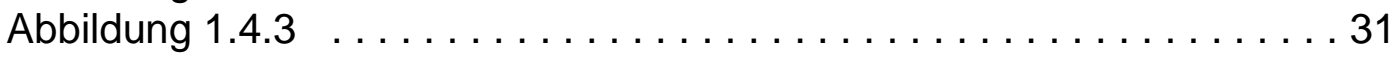

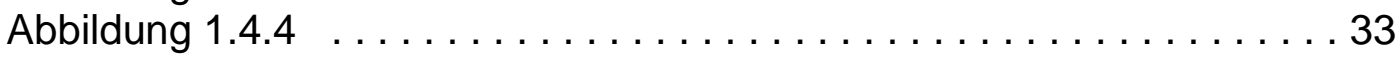

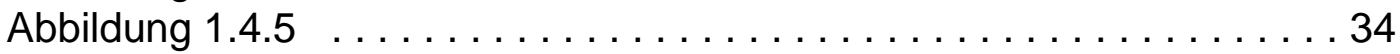

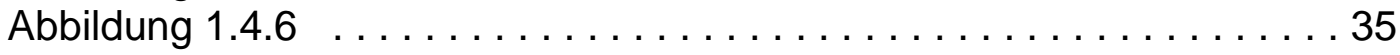

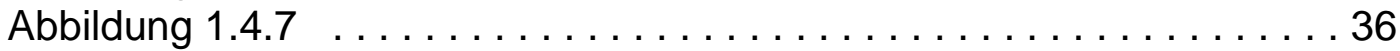

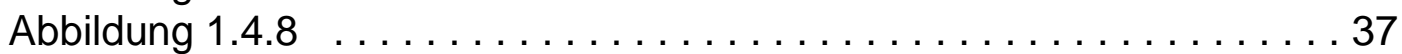

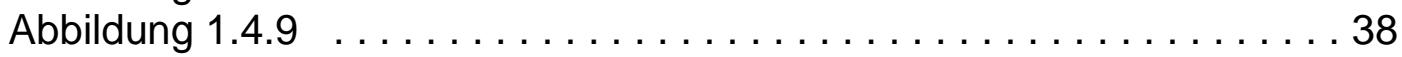

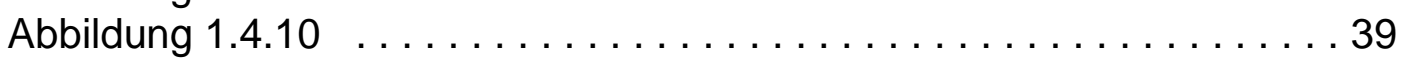

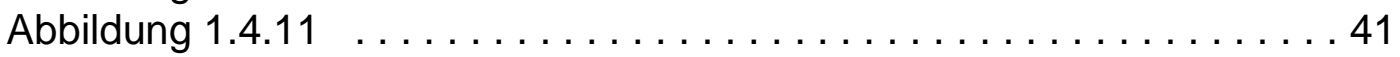

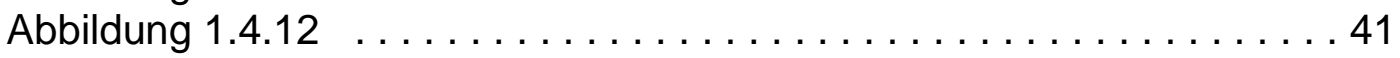

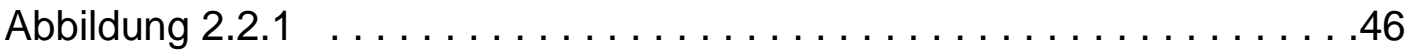

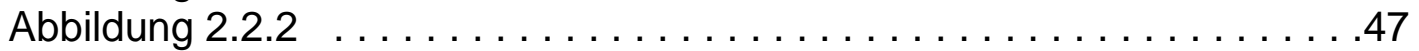




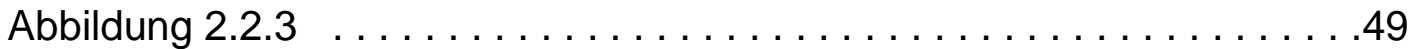

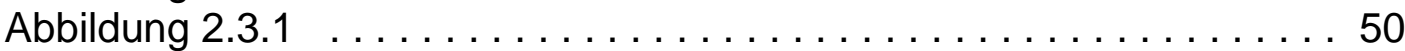

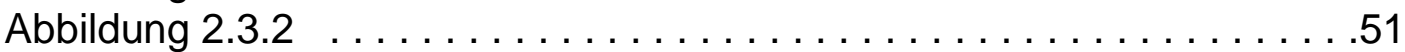

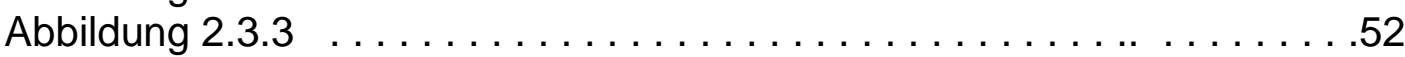

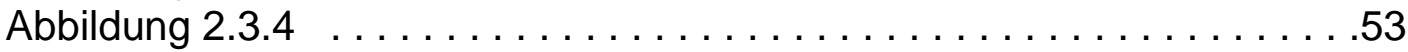

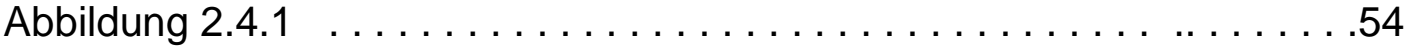

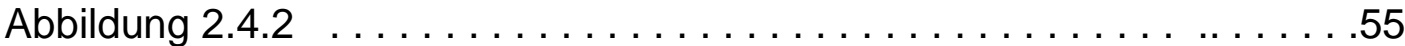

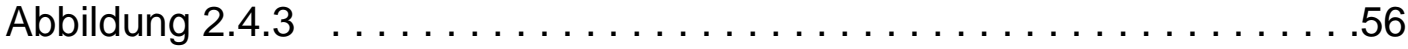

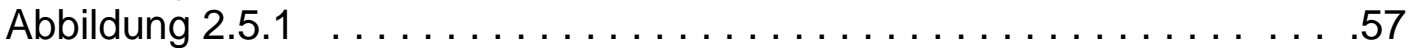

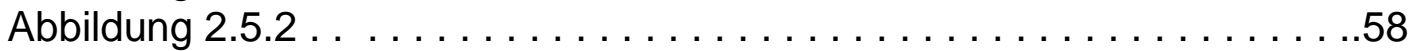

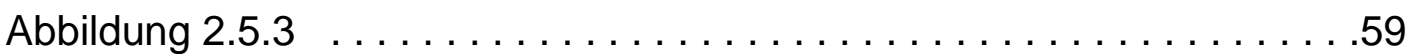

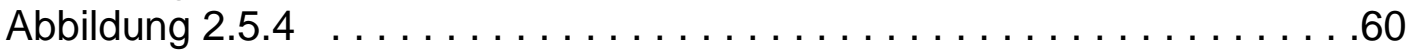

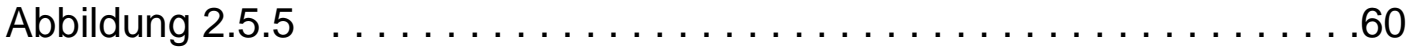

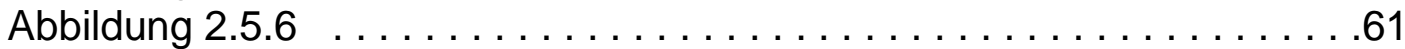

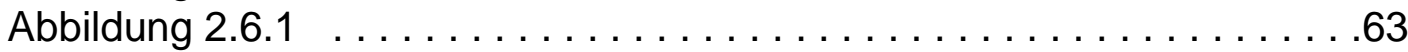

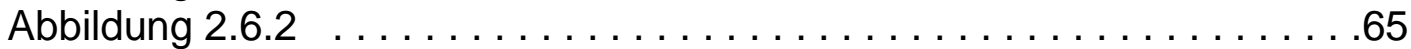

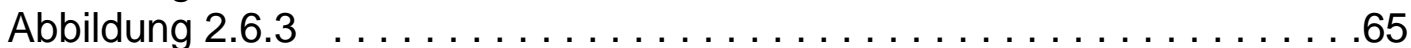

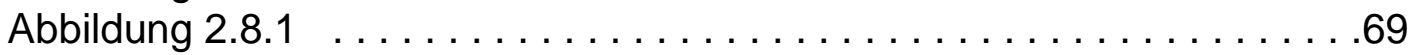

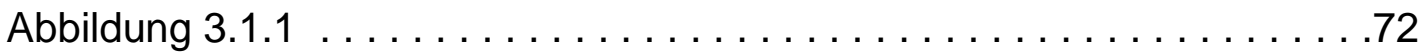

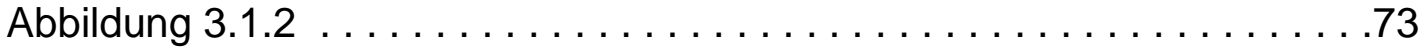

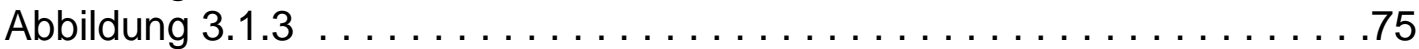

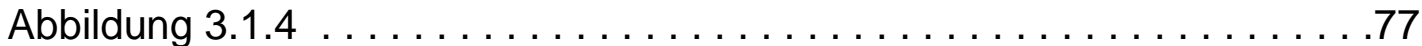

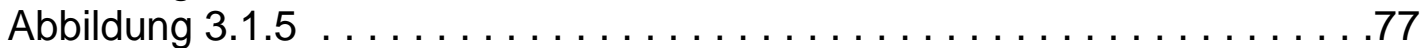

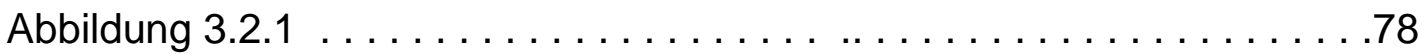

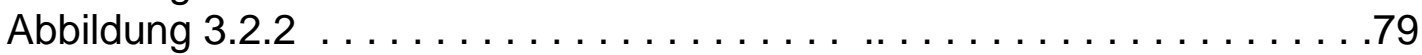

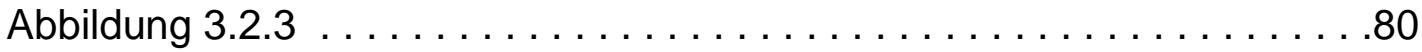

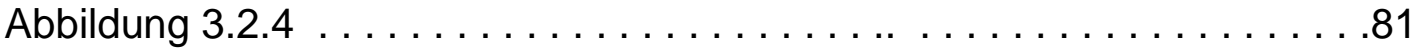

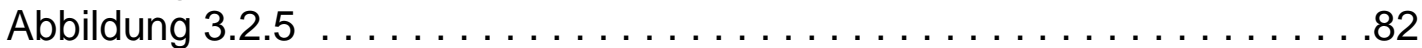

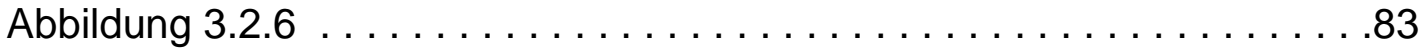

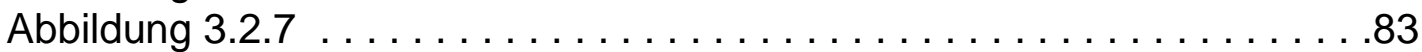

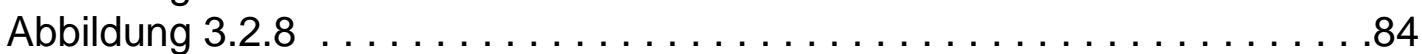

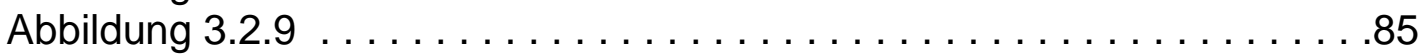

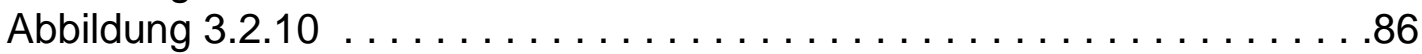

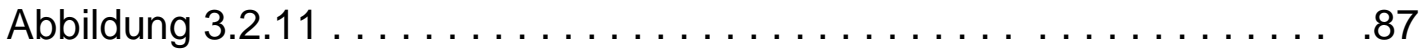

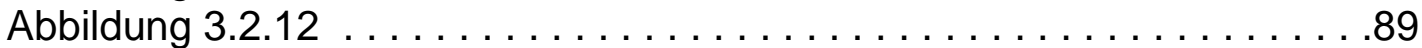

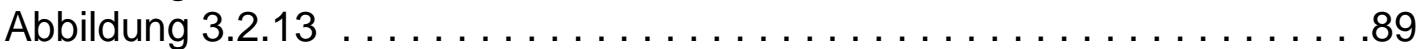

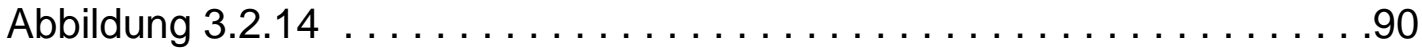

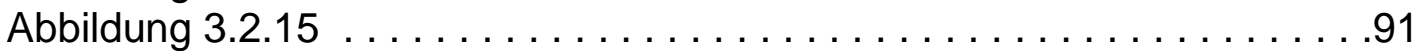

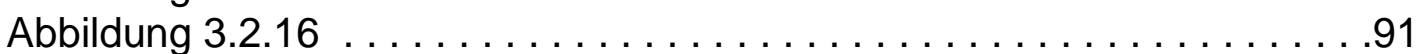

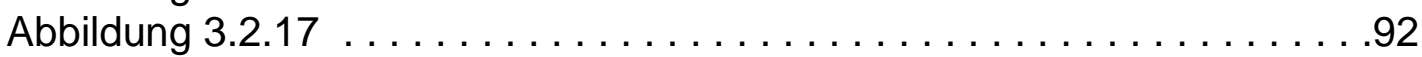

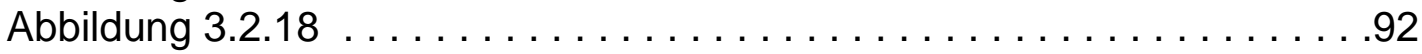

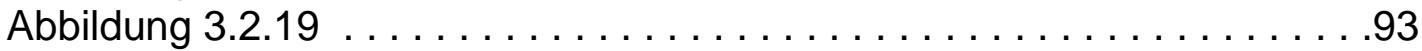

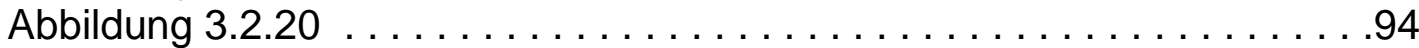

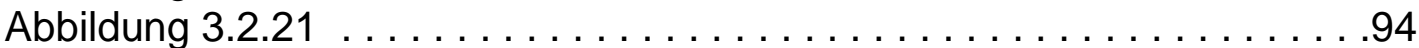




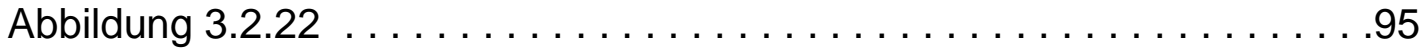

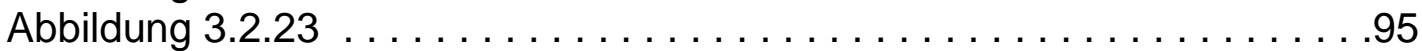

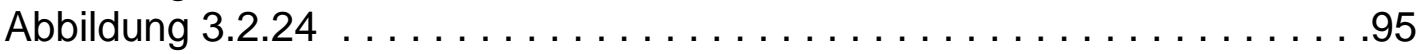

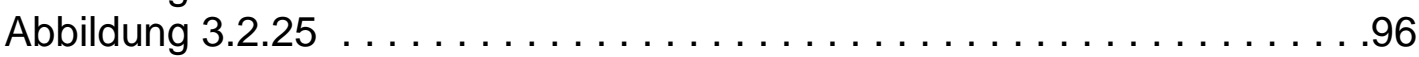

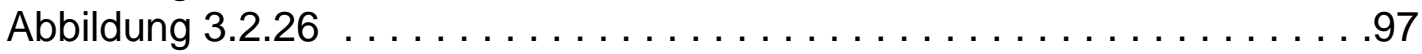

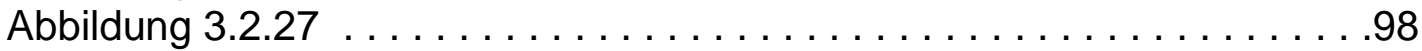

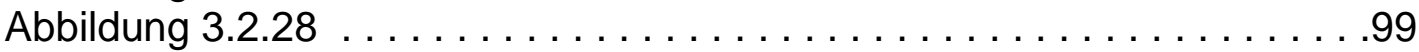

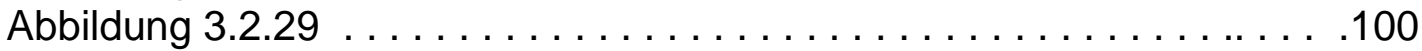

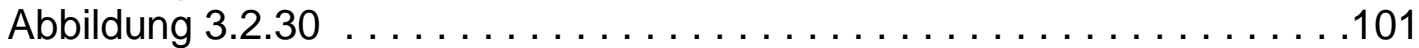

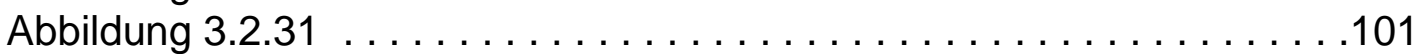

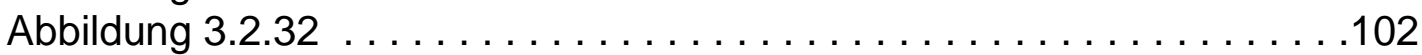

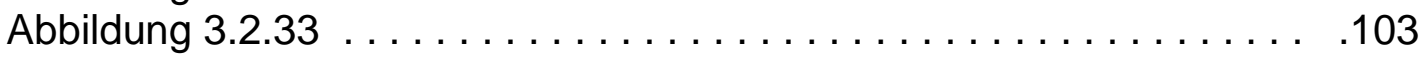

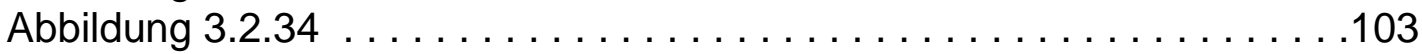

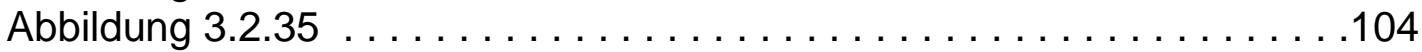

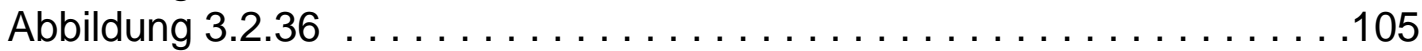

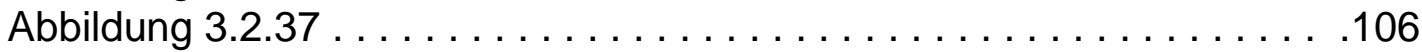

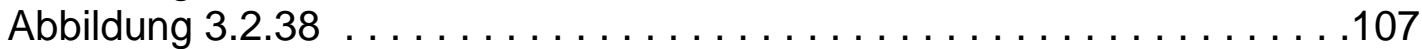

Abbildung $3.2 .39 \ldots \ldots \ldots$. . . . . . . . . . . . . . . . . . . . 108

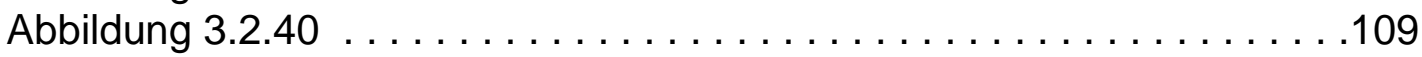

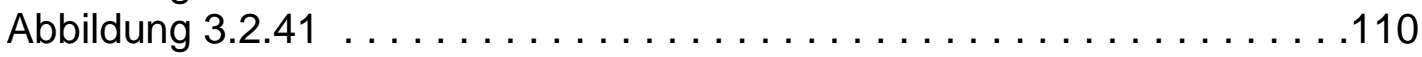

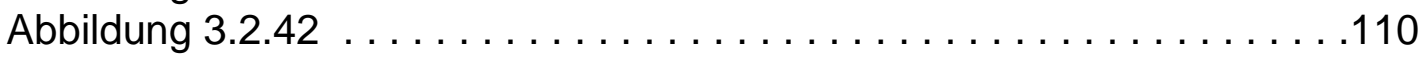

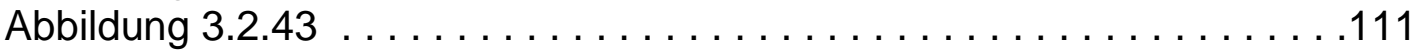

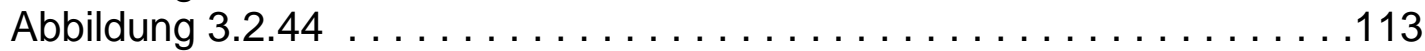

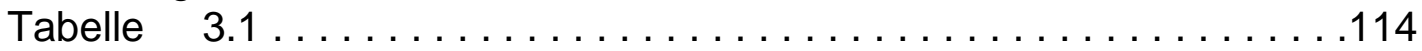

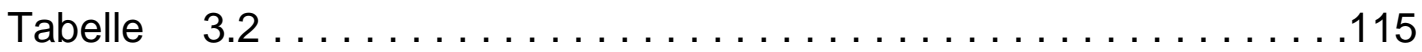

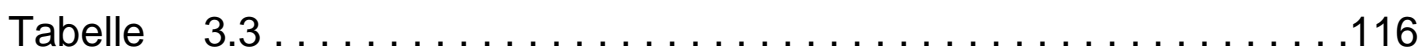

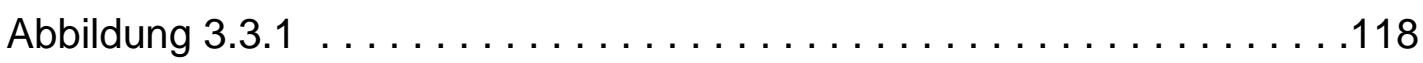

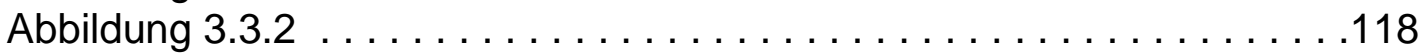

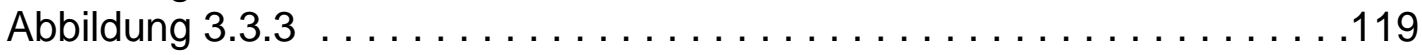

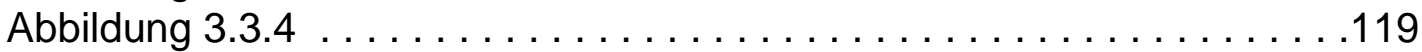

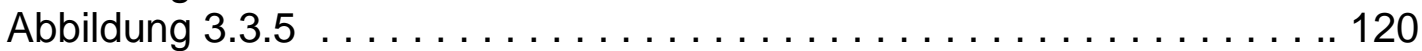

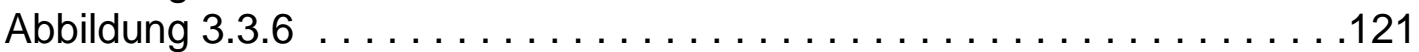

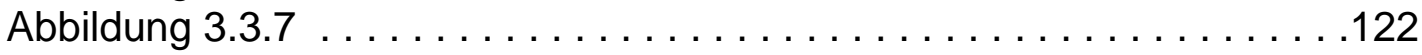

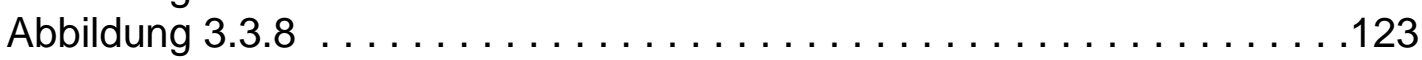

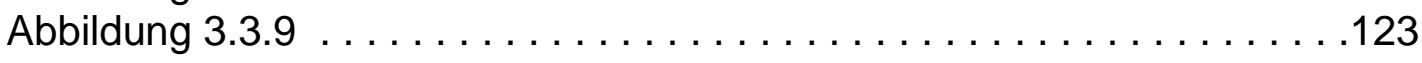

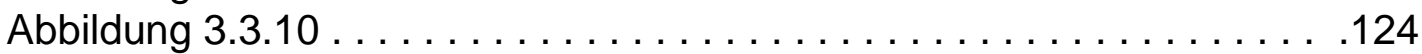

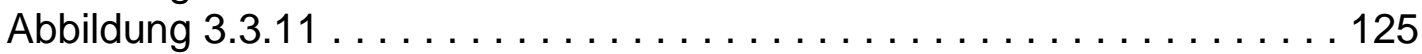

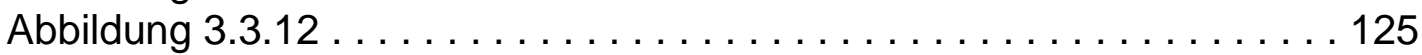

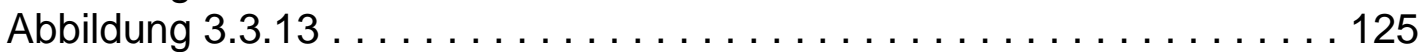

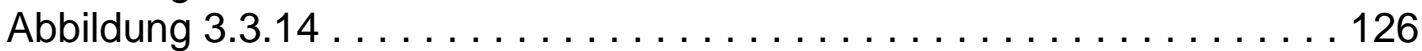

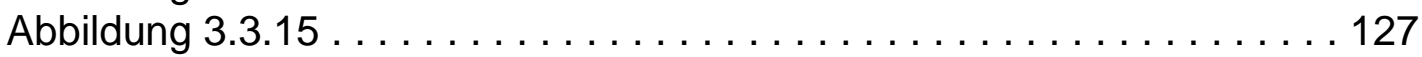

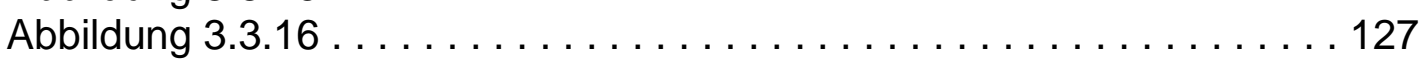

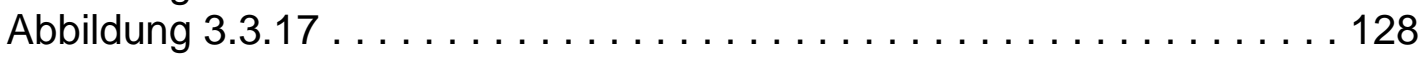

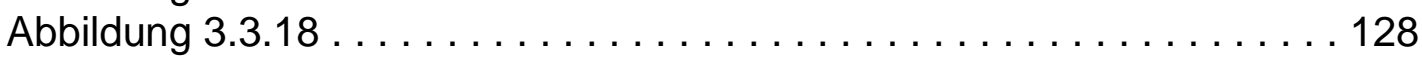

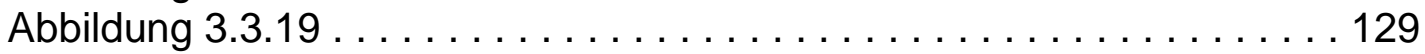

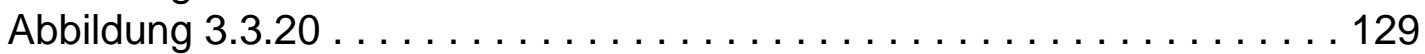




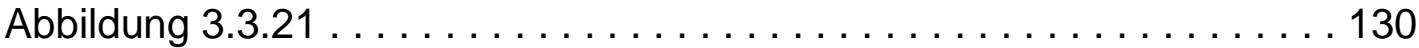

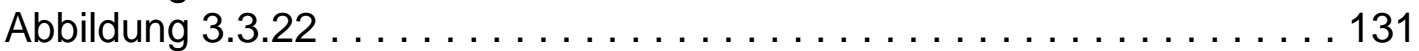

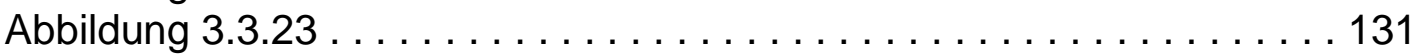

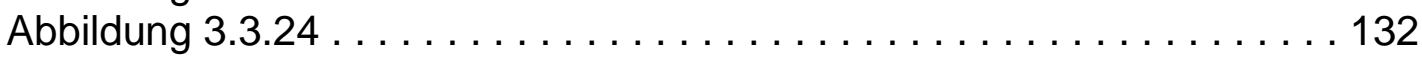

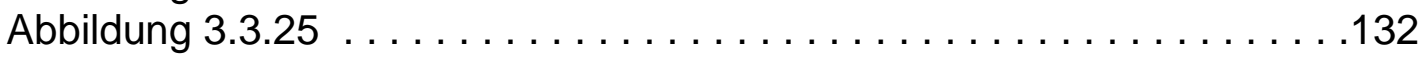

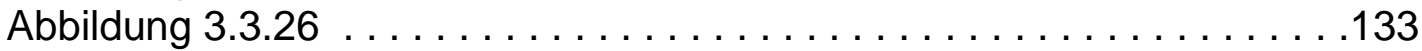

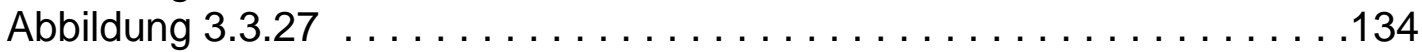

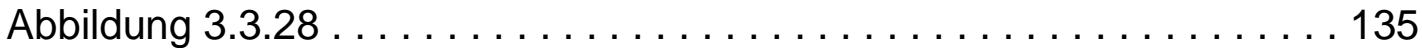

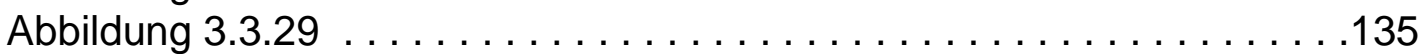

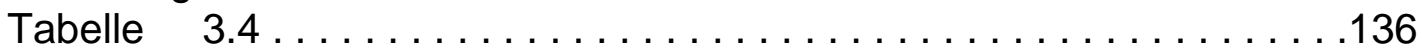

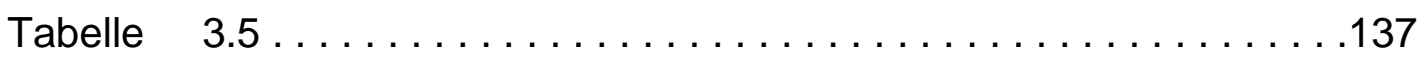

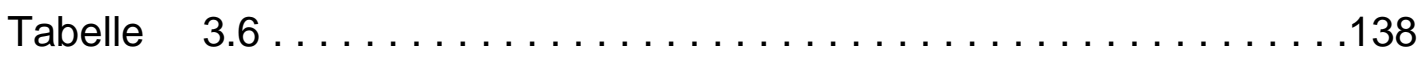

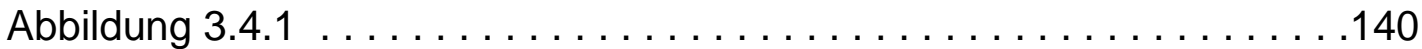

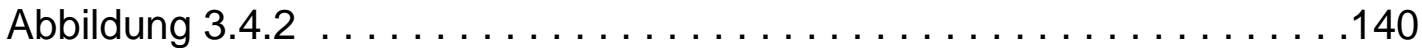

Abbildung $3.4 .3 \ldots \ldots \ldots \ldots \ldots$. . . . . . . . . . . . . . . . . . . . 141

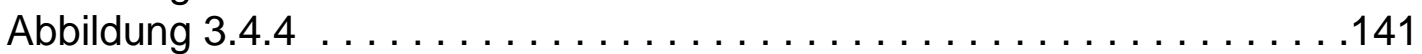

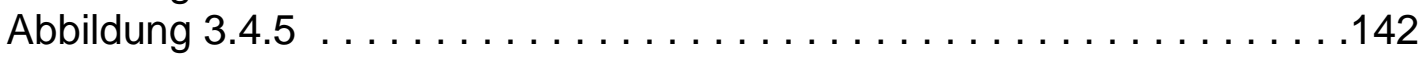

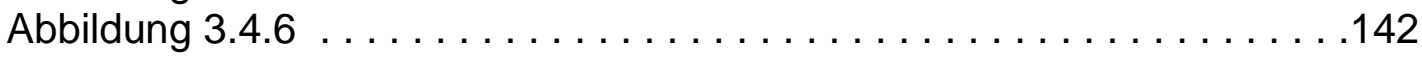

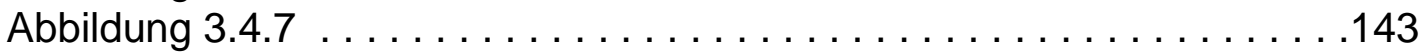

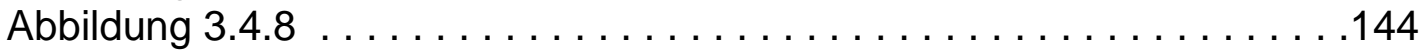

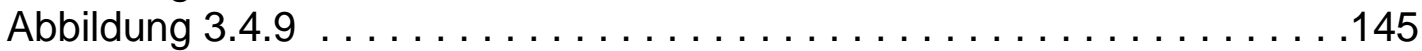

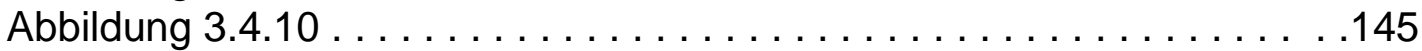

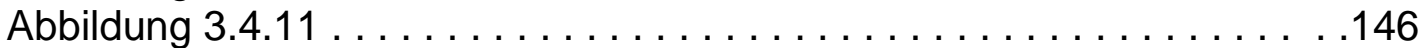

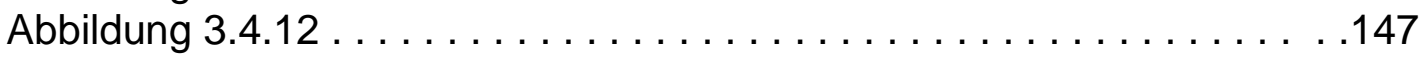

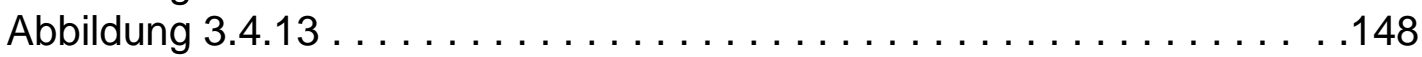

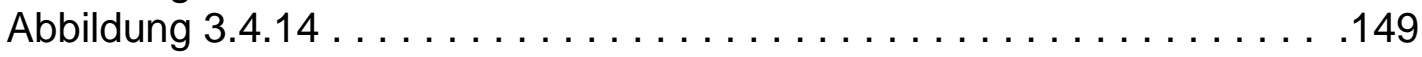

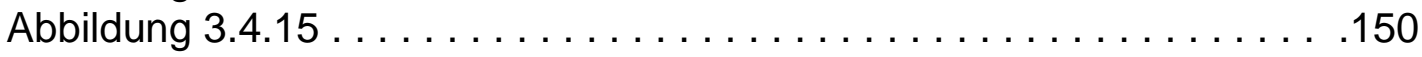

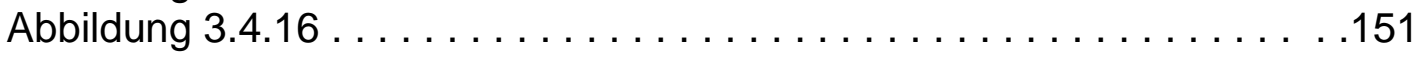

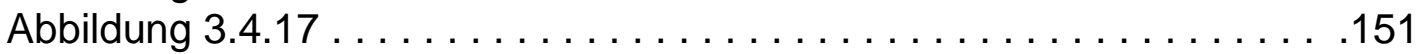

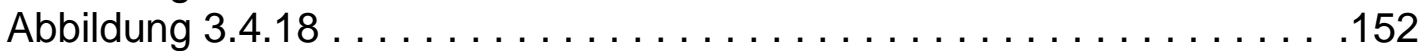

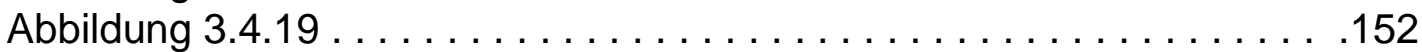

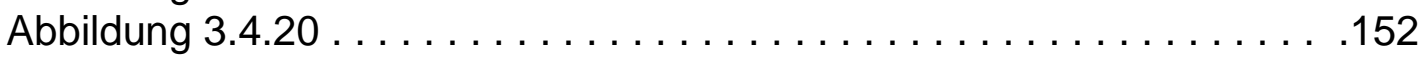

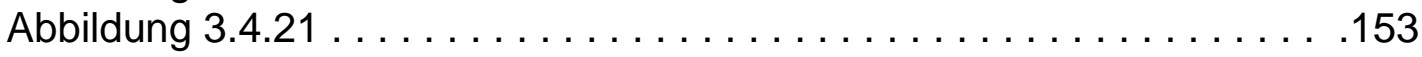

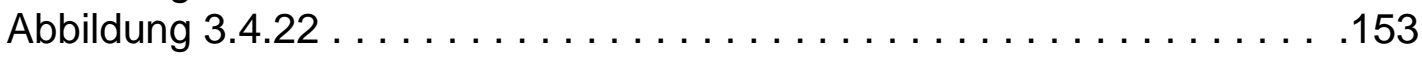

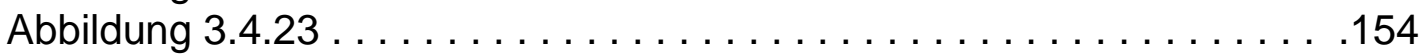

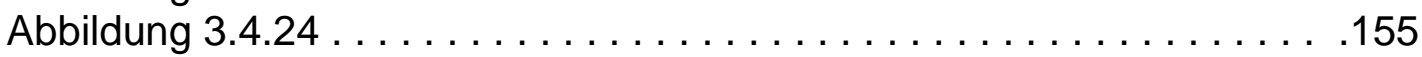

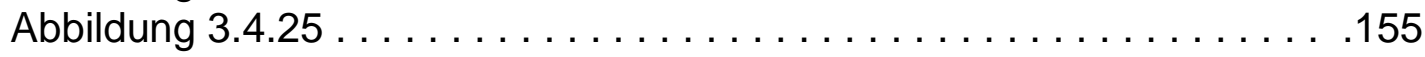

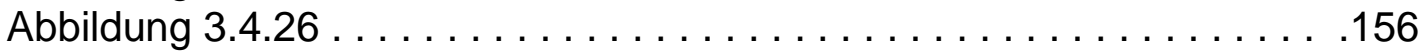

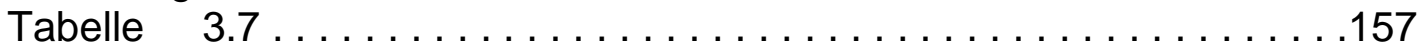

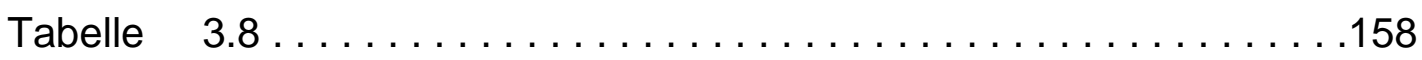

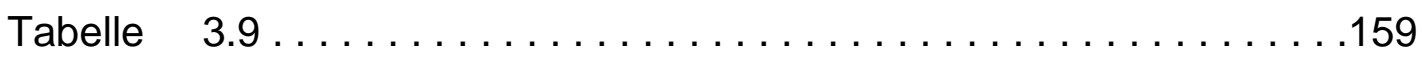




\section{Einleitung}

\subsection{Einführung}

Beschwerden und Erkrankungen der Wirbelsäule zählen zu den häufigsten Ursachen für Arztkonsultation und Arbeitsunfähigkeit. Die Rückenschmerzen gelten als eine aus epidemiologischer und sozialmedizinischer Sicht bedeutsame Gesundheitsstörung in den Industrienationen und verursachen erhebliche Kosten im Gesundheitssystem. So berichten in Deutschland mehr als 80\% der Erwachsenen mindestens einmal in ihrem Leben Rückenschmerzen gehabt zu haben (RASPE und KOHLMANN 1998). Die 30-40\% Befragten sprechen von Schmerzen „heute“ und 60-70\% von Rückenschmerzen „im letzten Jahr“. Im Alter von 20 Jahren haben schon mehr als $90 \%$ der Befragten die Rückenschmerzen erlebt (RASPE 2001). Das breite Spektrum von Ursachen reicht von akuten Ereignissen mit leichtgradigen Beschwerden und ohne merkliche Beeinträchtigung bis hin zu chronischen degenerativen Schäden mit ausgeprägter Funktionseinschränkung und erheblichen psychischen und sozialen Folgen (KOHLMANN ET. AL 1998). Das wurde auch von Seiten der Politik als ein volkswirtschaftliches Problem erkannt. Die Investitionen in die Entwicklung von neuen Präventionsmethoden, Verbesserung von Diagnostikverfahren und neuen Therapieansätzen bei Rückenschmerzen haben in den letzten Jahren enorm zugenommen.

Die Funktionsschäden und Instabilitäten im Bereich der Brustwirbelsäule sind im Vergleich zu den der Hals- und Lendenwirbelsäule wesentlich seltener. Die Bandscheibenschäden der Brustwirbelsäule spielen eher eine untergeordnete Rolle und machen lediglich 2\% der bandscheibenbedingten Erkrankungen der Wirbelsäule aus (KRÄMER UND GRIFKA 2005). Der Grund für so selten anzutreffende Krankheitserscheinungen im Brustwirbelbereich liegt sicher an der speziellen Anatomie und komplexen Biomechanik der thorakalen Segmente (Kapitel 1.2 und 1.4). Die Erkrankungen der Brustwirbelsäule, die relativ häufig 
auftreten und mit Hilfe der modernen Wirbelsäulenchirurgie sehr erfolgreich behandelt werden können, sind zum einen die therapieresistenten Nervenwurzelirritationen (Interkostalneuralgie) und zum anderen ausgedehnte Bandscheibenvorfälle, die das Rückenmark komprimieren und mit Lähmungserscheinungen einhergehen (KRÄMER UND GRIFKA 2007).

Die Fortschritte der Wirbelsäulenchirurgie in den letzten Jahrzehnten sind erheblich. Aufgrund der verbesserten Operationsverfahren und der Entwicklung moderner Implantate können traumatische Wirbelsäulenverletzungen, Bandscheibenschäden, sowie Tumorbehandlung erfolgreich behandelt werden. In letzter Zeit tritt auch die Entwicklung von neuen künstlichen Bandscheibenimplantaten immer mehr in den Vordergrund. Die Annäherung an die anatomischen Gegebenheiten von menschlichen Wirbelbogengelenken und das Nachahmen ihrer physiologischen Eigenschaften ist das Bestreben der Forscher. Für den langfristigen Erfolg einer operativen Versorgung ist jedoch auch die Wiederherstellung der biomechanischen Funktion von Bedeutung. Zurzeit sind die funktionserhaltenden Maßnahmen in der Wirbelsäulenchirurgie aufgrund der komplexen Biomechanik der Wirbelsäule und ihrer funktionellen Bestandteile nur begrenzt möglich.

Ohne die fundierten Kenntnisse in Anatomie, Physiologie und Pathophysiologie, ist eine normale Funktion der Wirbelsäule sowie die Diagnostik, Therapie und Prophylaxe von Wirbelsäulenerkrankungen nicht möglich (FARFAN 1973). Ein tieferes Verständnis von der Komplexität des Bewegungsapparates beinhaltet unter anderem die Betrachtung der biomechanischen Zusammenhänge der einzelnen Bauelemente, wie Knochen, Gelenke, Bänder und Muskeln und ihr funktionelles Zusammenwirken in einer physiologischen Bewegung. Deswegen besitzt die biomechanische Forschung und die Forschung der funktionellen Anatomie der Gelenke einen ausgeprägten interdisziplinären Charakter: Biologie und Medizin ergänzen sich wechselseitig mit der Getriebelehre und der technischen Mechanik. Es können also die Ideen aus der Technik zur Beschreibung der biologischen Funktionen genutzt werden.

Nichtsdestotrotz wird in der täglichen Anwendung in der Chirurgie oder in der Anatomie immer noch die von R. Fick (FICK 1910 a, b) aufgestellte Gelenksyste- 
matik verwendet. R. Fick, der, obwohl er von der anatomischen Inkongruenz der Gelenkflächen Kenntnis besaß, sie beschrieb, ihr aber keine mechanische Bedeutung beigemessen hat, reduzierte in seinem Konzept die biologischen Systeme auf formschlüssige Gelenke, wie sie auch in der Getriebetechnik bekannt sind. Seitdem scheint bei Anatomen das Problem der Kinematik der Gelenkfunktion weitestgehend gelöst zu sein. Diese Funktionsmechanismen wurden in nahezu alle Anatomielehrbücher aufgenommen (RAUBER und KOPSCH 1987, FRICK et. al 1992, WALDEYER und MAYET 2002, BENNINGHOF und DRENKHAHN 2003, LIPPERT 2003). Diese Anschauung ist heutzutage nicht mehr vertretbar. Zum ersten Mal stellten WALMSLEY (1928) und MACCONAILL (1953) die Überlegungen zur funktionellen Bedeutung der inkongruenten Krümmung an, die sie als notwendige Voraussetzung für Schmierung und Ernährung des Knorpels ansahen. Ihre kinematische Bedeutung wurde aber auch hier nicht erkannt. Die Inkongruenz der artikulierenden Flächen wurde als nur ein „Konstruktionsmerkmal“ der Gelenkflächen angesehen und als „Schlottrigkeit der Natur “ (FICK A. 1871 in NÄGERL 1990, S. 2) bezeichnet. Die biologischen Gelenke werden zu technischen Gelenken mit passgenauem Formschluss reduziert und nach der Zahl der Freiheitsgerade eingeteilt (RAUBER und KOPSCH1987, WALDEYER und MAYET 2002).

H. NÄGERL hat sich mit der biomechanischen Bedeutung der Inkongruenz befasst und eine allgemeine Theorie der Gelenke (NÄGERL 1990) zur Systematisierung entwickelt. Die Inkongruenz der Gelenkflächen macht diese Gelenke zu einem Musterbeispiel für die Anwendung der Theorie der dimeren Ketten. Die Theorie wurde auf die Diarthrosen (KUBEIN-MeESENBURG ET AL. 1990, NäGERL ET AL. 1992) und Synarthrosen (NÄGERL ET AL.1990) und deren Zusammenspiel angewendet. Die Kinematik wurde mit Hilfe der klassischen Getriebelehre analysiert (NÄGERL 1990, Kubein-MeEsenbuRg ET AL. 1991a) und unter anderem auf die Mechanik der Wirbelsäule übertragen (NÄGERL 1990, NÄGERL ET AL. 1992).

Für die Untersuchung der komplexen Zusammenhänge der Wirbelsäule definierte JUNGHANNS (1977) zwei benachbarte Wirbelkörper mit der dazwischen liegenden Bandscheibe als eine kleine Funktionseinheit, das Junghannssche Bewegungs- 
segment. Die Gesamtbewegung der Wirbelsäule setzt sich also additiv aus den einzelnen Bewegungen der Junghannsschen Segmente (JUNGHANNS 1977) zusammen. Dementsprechend sollte es genügen, die Kinematik und Statik der einzelnen Bewegungssegmente zu analysieren, um die Gesamtbewegung der Wirbelsäule zu beschreiben (NÄGERL ET AL. 1995).

Bei der Anwendung der von NÄGERL (1990) entwickelten allgemeinen Theorie der Gelenke auf die Bewegungssegmente der Wirbelsäule wird deutlich, dass sich die Bewegungsstruktur zweier fester Körper im Raum nicht durch eine einzige Schraubung, wie von PANJABI ET AL. (1986) vorgeschlagen wurde, beschreiben lässt; PANJABI ET AL. haben die kinematischen Messungen an Lendenwirbelsegmenten erst im Jahr 1981 theoretisch beschrieben und fünf Jahre später experimentell durchgeführt. Dabei wurde lediglich die Gesamtrotation eines Lendenwirbels unter Anlegen eines axialen Drehmoments nach 30 Sekunden festgehalten. Aus End- und Ausgangslage zweier benachbarter Wirbelkörper wurde so die Position der einzigen Schraubachse (Englisch: Instantaneous helical axis; IHA) berechnet. Diese Achse wurde als momentane Schraubachse bezeichnet. PANJABI ET AL. (1986) erfassten zwar richtigerweise dreidimensionale Bewegungen, verglichen aber nur Anfangs- und Endzustände einer Drehung, was keineswegs zur Beschreibung der tatsächlichen Bewegungsstruktur genügt. So ist festzuhalten, dass die Bewegungsstruktur nur als eine Abfolge mehrerer, enganeinander liegender, aber unterschiedlichen Schraubachsen beschrieben werden kann. Um die differentiellen Schraubachsen zu erfassen und die Bewegungsstruktur zu entschlüsseln, sind die dreidimensionalen „Momentaufnahmen" der Bewegung sowie Achsenberechnung mit Hilfe von differentiell kleinen Lageänderungen, d.h. Rotationswinkeln, notwendig.

Betrachtet man die Bewegungsstruktur zweier benachbarter Körper aus mathematischer und physikalischer Sicht, liegt die Mindestanforderung zur Auslösung des Bewegungsablaufes bei einer Auflösung von $0,2^{\circ}$ pro momentane Schraubachse und einem Bewegungsumfang von 1,50 (NÄGERL ET AL. 1995). In vielen Studien werden vor allem die Drehwinkel-Drehmoment-Kennlinien bzw. Last-Deformationskurven (WILKE 1993, MYERS ET AL. 1991, PANJABI ET AL. 1986) betrachtet. Dieser Parameter ist allein nicht aussagekräftig und soll in der 
vorliegenden Arbeit im Zusammenhang mit der Wanderung der momentanen Schraubenachsen zur Analyse der Bewegungsstruktur mit betrachtet werden.

Basierend auf der Theorie zur Biomechanik von Synarthrosen und Diarthrosen von H. NÄGERL (1990) wurde in der Biomechanischen Arbeitsgruppe der Universität Greifswald und Göttingen eine hoch auflösende Messtechnik entwickelt, die die komplexe Bewegungsstruktur eines Junghansschen Segmentes in sehr dichter Folge erfasst. Die Apparatur wurde von SPIERING (2002) entwickelt und es wurden zahlreiche biomechanische Untersuchungen an verschiedenen Wirbelbogensegmenten vorgenommen; an der Lendenwirbelsäule (MANSOUR 2001), an der Halswirbelsäule (KALSCHEUER 2001, BOCKERMANN 2004, WACHOWSKI 2005) und an der Brustwirbelsäule (LEE 2003).

Gestützt auf die oben aufgeführten Bedingungen ist das Ziel dieser Arbeit, den Bewegungsablauf im Bewegungssegment Thorakalwirbel 2 und 3 (Th2/3) zu analysieren und seine biomechanischen Bewegungseigenschaften vollständig, hochaufgelöst und präzise zu bestimmen. Eine Weiterentwicklung der Messapparatur wurde im Vorfeld der Experimente vorgenommen, so dass sie den gestellten methodischen Ansprüchen und den rechnerischen Leistungen heutzutage gerecht wurden. Das Auswertungsprogramm wurde von Spiering im Jahr 2008 graphisch überarbeitet und erweitert.

Es folgten die Messungen zur Überprüfung der Messapparatur. Der Messaufbau wurde weiterentwickelt und die Messkette an die Begebenheiten des Brustwirbelkörpers angepasst. Es wurden Parameter definiert, um die biomechanischen Eigenschaften zweier Brustwirbelsegmente (Th2/3) zu vergleichen und zu interpretieren.

Der Inhalt und strukturelle Aufbau der vorliegenden Arbeit ist so gewählt, dass eine verständliche und überschaubare Einführung in die Biomechanik der Brustwirbelsäule am Beispiel eines oberen thorakalen Bewegungssegments ermöglicht wird. 
Das erste Kapitel 1.2 Anatomie der Wirbelsäule verschafft einen Überblick über Aufbau und Funktion der Wirbelsäule im Ganzen und speziell der Brustwirbelsäule. Ihre anatomischen Komponenten werden erläutert und verschaffen so einen Bezug zu den Ergebnissen der bekannten Morphologie. In dem folgenden Kapitel 1.3 Mathematische und physikalische Grundlagen werden die für diese Arbeit nötigen Begriffe der Mechanik vorgestellt. Es werden die Grundbegriffe der Getriebelehre erläutert, um eine Einführung in das Konzept der differentiellen Schraubachsen und Bewegungen sowie der Kraftschrauben zu ermöglichen. Die Beschreibung der biomechanischen Prinzipien von Synarthrosen und Diarthrosen und ihre Wechselwirkung erfolgen im Kapitel 1.4 Biomechanik komplexer Gelenke. Basierend auf der von Nägerl und Kubein-Meesenburg aufgestellten Theorie der dimeren Ketten wird die Führungsfunktion der Gelenkflächen für die Bewegungsstrukturen unter verschiedenen Lastbedingungen erläutert. Die weiterentwickelte Messapparatur mit dem zugehörigem Messsystem ist im Kapitel 2. Material und Methoden dargestellt. Die Präparation von Brustwirbelkörpern Th 2/3 wird gezeigt und die einzelnen Komponenten des Versuchsaufbaus beschrieben. Im gleichen Kapitel wird das Messprinzip erläutert und die standardisierte Versuchsdurchführung vorgestellt. Das Kapitel 3. Ergebnisse beinhaltet die Erläuterungen initialer Messungen zur Kalibrierung der Messapparatur und beschreibt die experimentellen Ergebnisse der morphologischen, statischen und kinematischen Untersuchung der Brustwirbelsegmente (Th2/3). Bei den statischen Messungen wird die Positionsveränderung nach Aufbringen von unterschiedlichen axialen Vorlasten analysiert, wobei der Kraftangriffspunkt in sagittaler und transversaler Richtung systematisch variierte, sowie drei Rotationsarten um die drei Raumrichtungen ( „Axialrotation“, „Flexion/ Extension“ und „Lateralflexion“) durchgeführt. Die gewonnenen Daten werden aufgezeichnet und graphisch dargestellt. Es folgte dann die sukzessive Resektion beider Artt. zygapophysiales. Die Messungen werden unter gleichen Bedingungen wiederholt. Die dynamischen Messungen an den Bewegungssegmenten und die sukzessive Resektion der Wirbelbogengelenke bei systematischer Variation der Versuchsparameter sollten die biomechanischen Funktionen der Wirbelbogengelenke verdeutlichen. Am Ende werden die kompletten Messergebnisse zusammengefasst. Im Diskussionsteil 
dieser Arbeit, Kapitel 4. Diskussion, wird die angewandte Untersuchungsmethodik diskutiert. Anhand des beschriebenen biomechanischen und dieser Studie zugrundeliegenden physikalischen Modells werden die gefundenen Bewegungsstrukturen interpretiert und einer kritischen Wertung unterzogen. 


\subsection{Anatomie der Wirbelsäule}

\subsubsection{Allgemeine Anatomie}

\subsubsection{Füllgelenke (Synarthrosen)}

Die nicht unterbrochene Verbindung zweier Knochen durch Füll- und Bindegewebe bezeichnet man als Knochenfuge mit einem Füllgelenk (Synarthrose). Nach der Art des die Verbindung herstellenden Füllgewebes werden Synarthrosen in Syndesmosen (Articulatio fibrosa) mit einer Kontinuität aus straffem kollagenem oder elastischem Bindegewebe und in Synchondrosen (Articulatio cartilaginea) mit Knorpelfuge unterteilt. Man spricht von einer Synostose oder Knochennaht, wenn das ursprüngliche Bindegewebe verknöchert.

\subsubsection{Gelenke (Diarthrosen)}

Echte Gelenke bezeichnet man als Diarthrosen (Articulatio synovialis), bei denen die gelenkig verbundenen Skelettanteile durch einen Spalt von einander getrennt sind (Abb.1.2.1). Allgemein bezeichnet man ihn als Gelenkhöhle (Cavitas articularis). Der Gelenkspalt ist mit einer muzinhaltigen Flüssigkeit (Synovia) angefüllt. Die hyalronsäurereiche, visköse Substanz setzt die Reibung an den Gelenkflächen herab und spielt bei der Ernährung des Gelenkknorpels, der die Skelettanteile bedeckt, eine wichtige Rolle. Der hyaline Gelenkknorpel (Cartilago articularis) ermöglicht mit seiner glatten Oberfläche ein reibungsarmes Gleiten der Gelenkkörper. Die Gelenkflächen werden durch eine kompressive Kraft in Funktion gesetzt. Sie treten dabei in Kontakt, wobei die Größe der Kontaktfläche mit zunehmender

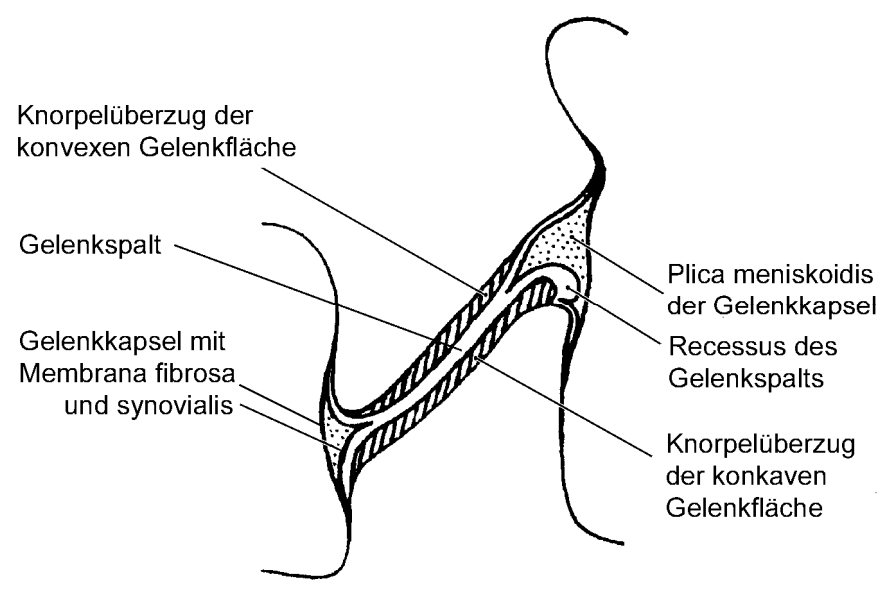

Abb. 1.2.1: Schema einer Diarthrose

Kraft anwächst. Die Gelenkkapsel (Capsula articularis) umschließt die Gelenkhöh- 
le. Sie besteht aus zwei Faserschichten. Einer äußeren (Membrana fibrosa) und einer inneren synovialen Deckschicht (Membrana synovialis), deren Zellen an der Bildung der Synovia beteiligt sind. Die Krümmung der beiden Gelenkflächen ist inkongruent (FICK 1910a, b). Die Inkongruenz der artikulierenden Gelenkflächen ist ein wichtiges funktionelles Konstruktionsmerkmal aller biologischen Gelenke (NÄGERL 1990).

\subsubsection{Anatomie der Wirbelsäule}

\subsubsection{Wirbelsäule als Ganzes}

Die Wirbelsäule, das Rückgrat, ist die gegliederte und bewegliche Stütze des Rumpfes. Die einzelnen Glieder dieser Säule nennen wir Wirbel, Vertebrae. Das Vorhandensein der Wirbelsäule hat der großen Gruppe der Wirbeltiere den Namen gegeben (WALDEYER und MAYET 2002).

Man unterscheidet nach der jeweiligen Lage vor allem nach den anatomischen Besonderheiten drei Abschnitte. Die Halswirbelsäule (HWS) mit 7 zervikalen Wirbeln und Kontakt zur Schädelbasis, die Brustwirbelsäule (BWS) als Ursprung der Rippen mit 12 thorakalen Wirbeln und die Lendenwirbelsäule (LWS) mit 5 Wirbeln. Die kaudal folgenden 5 Kreuzbeinwirbel sind zu einem einheitlichen Kno-

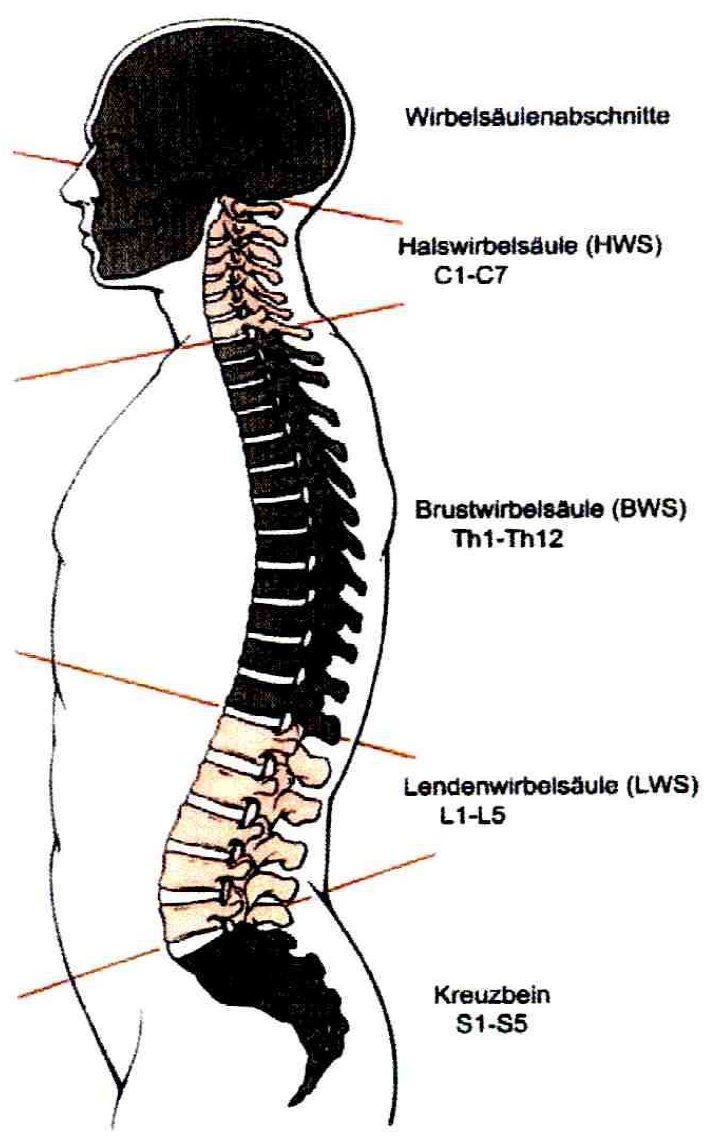

Abb. 1.2.2: Übersicht der Wirbelsäulenregionen (NiethaRd Und Pfeil 1989, S. 293) chen verschmolzen, dem Kreuzbein (Os sacrum).

Die Wirbelsäule trägt die Last von Kopf, Rumpf und oberen Extremitäten und verleiht dem Körper sowohl Halt als auch seine umfangreiche Beweglichkeit. Ihre typische S-Form beruht auf den vier charakteristischen Krümmungen (Abb. 1.2.2). 
Im Hals- und Lendenbereich ist sie nach ventral konvex, die so genannte Lordose, im Brust- und Kreuzbeingebiet konkav; die Kyphose.

Diese Krümmungen zusammen mit elastischen Eigenschaften der Bänder und Bandscheiben führen zu den mechanisch-stabilen Federeigenschaften der Wirbelsäule und ermöglichen somit die Aufnahme und Weiterleitung von Stößen. Gleichzeitig hat die Wirbelsäule eine Schutzfunktion für das im Wirbelkanal (Canalis vertebralis) verlaufende Rückenmark und die Spinalnervenwurzeln. Der Aufbau der Wirbelsäule aus verschiedenen knöchernen und bindegewebigen Elementen bildet die morphologische Grundlage für die komplexen statischen, kinematischen und elastischen Funktionen.

Die Gesamtbewegung der Wirbelsäule setzt sich aus der Summe der Einzelbewegungen der Segmente zusammen (JUNGHANNS 1977). Die kleinste funktionelle Einheit, das Junghannssche Bewegungssegment, besteht aus zwei Wirbeln, der dazwischen liegende Bandscheibe und dem Bandapparat (JUNGHANNS 1977).

Die Bewegung in diesem Segment ist gering. Die Gesamtbewegung der Wirbelsäule ist aber sehr umfangreich. Sie erfolgt in allen Ebenen des Raumes.

Die Hauptbewegungen sind:

- Flexions-/Extensionsbewegung

- Lateralflexion

- Axiale Rotation.

\subsubsection{Allgemeines zur Entwicklung der Wirbelsäule}

Die Wirbelsäule ist eine Mittellinien-Struktur und ihr Wachstum spielt sich vorzugsweise in kranio-kaudaler Richtung ab (FARFAN und ERDMANN 1979). Sie entwickelt sich aus einer Mesenchymscheide um die Chorda dorsalis, der embryologischen Körperachse.

Die Wirbel entwickeln sich aus einer unteren und einer oberen Hälfte benachbarter Mesenchymsegmente. Die Zwischenwirbelscheibe geht jeweils aus der oberen Hälfte der Wirbelanlage hervor (SCHIEBLER u. KORF 2007). Der gallertartige Kern (Nucleus pulposus) der Bandscheibe wird als Überrest der Chorda dorsalis angesehen. 
Die Wirbelbögen gehen aus nach dorsal gerichteten Neuralfortsätzen der Wirbelanlage (Processus dorsalis) hervor. Sie umschließen die Anlage des Rückenmarks bis zur Vereinigung zum Dornfortsatz (Processus spinosus). Nach lateral wächst sich der nach ventral gerichtete Teil der Wirbelanlage zum Rippenfortsatz aus. Die Querfortsätze (Processus transversı) bleiben zunächst weitgehend von den Rippenanlagen getrennt und verbinden sich erst im Laufe ihres Längenwachstums miteinander. Zwischen Processus transversi und Rippen entstehen Gelenkspalten.

Die Wirbelbogengelenke entwickeln sich durch die Angliederung, d.h. durch das Aneinanderlegen ursprünglich getrennter Skelettanteile. Als Anlage der Gelenkspalte entsteht an den Berührungsstellen der beiden Gelenkkörper eine Art Schleimbeutel, der aber rasch die Eigentümlichkeit eines echten Gelenks annimmt (TÖNDURY 1958). Die Gelenkflächen sind zunächst dachziegelartig übereinander ausgerichtet und frontal gestellt. Das ändert sich im Lendenwirbelbereich postnatal, wobei sie eine sagittale Ausrichtung einnehmen. Im Brustwirbelbereich ändert sich die Ausrichtung nicht und es bleibt die dachziegelartige Anordnung der Gelenkflächen erhalten (LUTZ 1967).

Die folgenden Abschnitte beschreiben den anatomischen Aufbau der Anteile der Wirbelsäule, die ein Bewegungssegment nach Junghanns darstellen (zwei benachbarte Wirbeln, Bandscheibe und Bandapparat).

\subsubsection{Wirbel}

Die Wirbel (Vertebrae) sind die knöchernen Bauelemente der Wirbelsäule. Jeder Wirbel (Abb. 1.2.3) besteht aus einem zylinderförmigen Wirbelkörper (Corpus vertebrae) und einem schlanken, knöchernen Umbau des Wirbelkanals (Foramen vertebrale), dem Wirbelbogen (Arcus vertebrae). Von den Wirbelbogen gehen mehrere Fortsätze ab. Man unterscheidet zwei Gelenkfortsätze (Processus articulares), zwei Querfortsätze (Processus transversi) und den unpaaren Dornfortsatz (Processus spinosus) als Hebelarme für die ansetzende Muskulatur. Der Wirbelkörper hat eine dünne, kompakte Außenschicht und eine starke Spongiosa (WALDEYER und MAYET 2002). Die Randleisten der Ober- und Unterseite 
springen wulstartig hervor. Die axialen Endflächen der Wirbelkörper sind dementsprechend konkav gekrümmt und mit einer feinen hyalinknorpeligen Abschlussplatte bedeckt. Die zwei benachbarten Wirbelkörper werden durch eine Synchondrose, die Zwischenwirbelscheibe (Discus intervertebralis), miteinander verbunden.
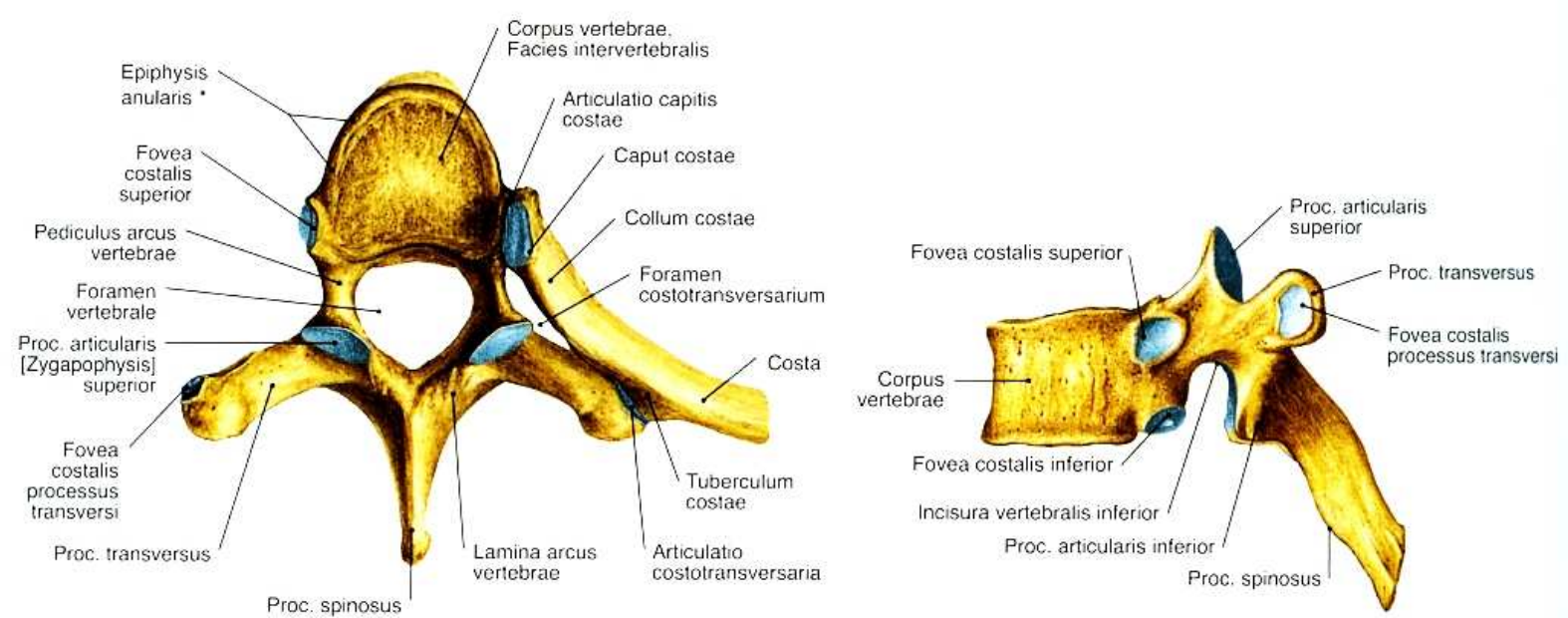

Abb. 1.2.3: Brustwirbel von kranial und von links (SОвотTA 2007, S.266)

Die meisten Körper der Brustwirbel tragen lateral eine kleine obere und eine untere halbe Gelenkfläche, die die Verbindung mit dem Rippenkopf darstellt (Fovea costalis superior und inferior). Der Wirbelbogen ist dort, wo er vom Körper abgeht, am oberen Rande leicht, am unteren tief eingeschnürt (Incisurae vertebrales superior und inferior). Diese Einschnürungen der oberen und der unteren Wirbel ergänzen sich zu einem Zwischenwirbelloch (Foramen intervertebrale). Die Größe und Form der Foramina intervertebralia variieren in den einzelnen Abschnitten der Wirbelsäule. Die Größe nimmt von oben nach unten zu. Ihre engste Stelle liegt zwischen dem 4. und 6. thorakalen Wirbel (KRÄMER und GRIFKA 2005). Ihre pathologische Einengung ist eine nicht seltene Erkrankung, die zu Druckerscheinungen an den dort austretenden Rückenmarksnerven und zu Störungen in deren Innervationsgebiet führt.

Die Gelenkfortsätze bestehen aus 2 oberen und 2 unteren mit Knorpel überzogenen Gelenkflächen (Processus articulares superiores und inferiores). Die oberen Gelenkflächen sind oval und in transversaler Richtung plan oder leicht konvex, sie weisen bei den Brustwirbeln nach dorsolateral, die unteren Gelenkflä- 
chen nach ventromedial. Die unteren Gelenkflächen artikulieren mit den superioren Gelenkflächen des kaudal benachbarten Wirbels.

Die Querfortsätze (Processus transversi) tragen bei den 10 kranialen Brustwirbeln eine Gelenkfläche (Fovea costalis transversalis) zur Verbindung mit dem Rippenhöckerchen (Tuberculum costae) (WALDEYER und MAYET 2002).

\subsubsection{Wirbelbogengelenke (Articulationes zygapophysiales)}

Die Artt. zygapophysiales (Facettengelenke) sind Diarthrosen. Die Gelenkkapsel ist im Bereich der Brustwirbelgelenke straff sowie durch Bandzüge verstärkt. Meniskoide Falten der Gelenkkapsel (PUTZ 1981, ČIHAK 1981), auch Plicae meniscoides genannt, ragen keilförmig in den Gelenkspalt (Abb. 1.2.1, S. 8) und bewirken ein „Klaffen“ (PUTZ 1981, NäGERL ET AL. 1990) des Gelenkspalts durch die auffällige Inkongruenz der Krümmung der artikulierenden Gelenkflächen (ZUKSCHWERDT ET AL. 1960). Die Gelenkflächen der Brustwirbel ähneln ungleichmäßig gekrümmten Halbzylindern, die nach vorne orientiert sind. Die konvexen Gelenkflächen (Facies art. inf.) des unteren Wirbels umfassen die konkaven (Facies art. sup.) des oberen Wirbels von außen. Die Anatomie der Wirbelgelenke wurde auch von PANJABI ET AL. (1991) ausführlich beschrieben.

\subsubsection{Zwischenwirbelscheibe (Discus intervertebralis)}

Die Bandscheiben (Discus intervertebralis) verbinden die Wirbelkörper benachbarter Wirbel vom zweiten Halswirbel bis zum Kreuzbein. Sie nehmen an Höhe und Querschnitt von den oberen zu den unteren Wirbelsäulenabschnitten zu (FARFAN 1979).

Sie sind an der typischen Wirbelsäulenkrümmung direkt beteiligt. So sind sie im Hals- und Lendenabschnitt vorne und im Brustbereich hinten höher (BOGDUK und TWOMEY 1991).

Eine Bandscheibe (Abb. 1.2.4 A, B, S. 14) besteht aus dem Faserring (Anulus fibrosus) und dem zentral gelegenen Gallertkern (Nucleus pulposus). Der Anulus fibrosus besteht aus 10-20 konzentrisch geschichteten Bindegewebslamellen (Bogduk und TWOMEY 1991) und Faserknorpel. Die Fasern entspringen bzw. setzen an Deckplatte und Boden der Wirbelkörper an. Durch ein Kreuzen (Abb. 
1.2.4 C) der benachbarten Kollagenfaserbündel entsteht ein dichtes scherengitterartiges Geflecht (KAPANDJI 1985). Der Nucleus pulposus setzt sich im Wesentlichen aus einer homogenen Proteoglykanmatrix mit circa 90\% Wasseranteil (KRÄMER 1987, GÖTZ ET AL. 1996, GöTZ ET AL. 1999) zusammen. Der Gallertkern hat aufgrund seines Glykosaminglykananteils einen hohen kolloidosmotischen Druck und besitzt die Fähigkeit, Wasser zu binden. Der Gallertkern ist von einer peripheren, fibrillären Hülle umgeben und gefäß- und nervenfrei.

\section{Discus intervertebralis}

A

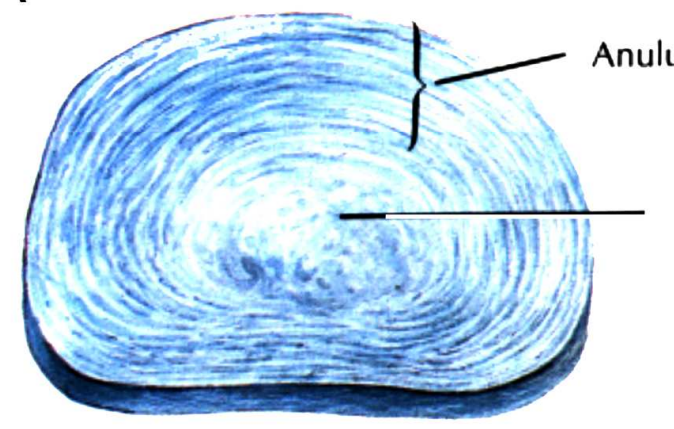

B

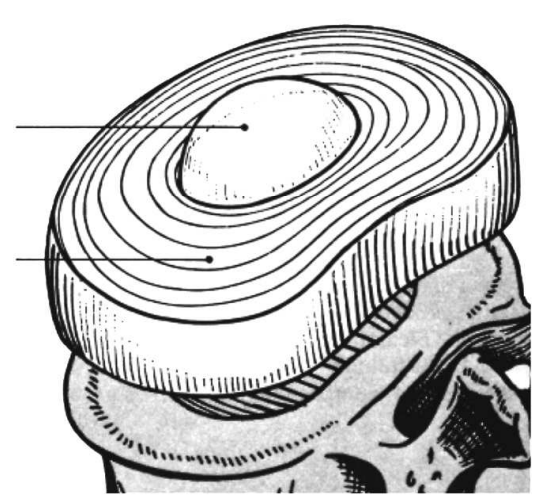

Nucleus

pulposus

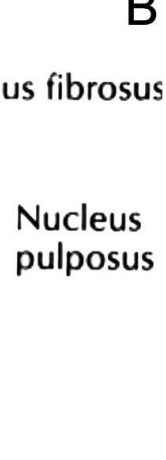

C

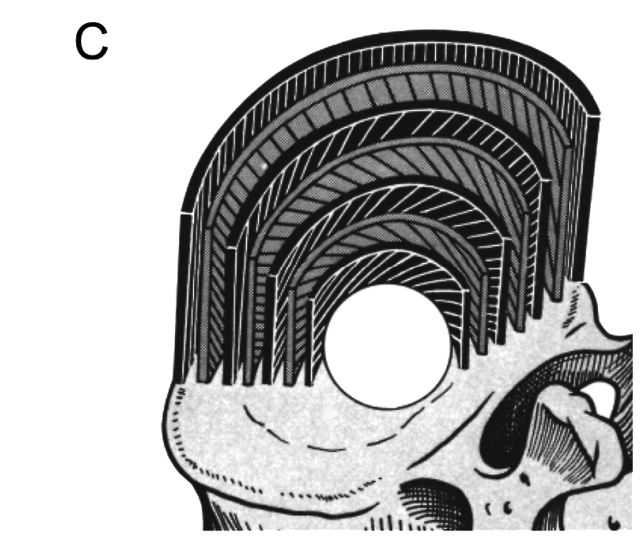

Abb.1.2.4: Zeichnung einer Bandscheibe (Discus vertebralis) im Querschnitt (A, NETTER 2003, S.148), als Schema mit Lamellenstruktur der Fasern von kraniolateral (B). Scherengitterartiger Verlauf der Faserbündel einer Lamelle (C, KAPANDJI 1985, S. 24).

Der große Wasseranteil des Nucleus pulposus ist sowohl vom Umgebungsdruck als auch vom Alter abhängig und trägt maßgeblich zu seiner Beweglichkeit bei (WASSILEV und KÜHNEL 1992). Mit dem im zunehmenden Lebensalter unmittelbaren Proteoglykanverlust vermindert sich der Wassergehalt des Nucleus pulposus, Elastizität und Verformbarkeit nehmen ab. 
Der verschiebbare inkompressible Nucleus ähnelt einem Wasserkissen. Er sorgt für eine gleichmäßige Druckverteilung und die andauernde Anspannung des Faserrings. Der Anulus fibrosus sichert die bindegewebige Verbindung der Wirbelkörper und positioniert den Nucleus. Bei Rotation verschnüren die gekreuzten Fasern den Gallertkern. Der inkompressible Gallertkern verformt sich und schiebt die Wirbelkörper auseinander. Es resultiert eine Höhenzunahme der Bandscheibe unabhängig von der Rotationsrichtung (NÄGERL ET AL. 1995).

\subsubsection{Bänder der Brustwirbelsäule}

Die kräftigen Bindegewebsfasern verbinden zusätzlich zur Bandscheibe die Wirbelkörper miteinander.

Der Bandapparat (Abb. 1.2.5) besteht aus einer Vielzahl von Ligamenten. Erstens aus kräftigen Längsbändern an der Vorder- und Rückseite der Wirbelkörper. Das vordere Längsband (Ligamentum longitudinale anterius) erstreckt sich auf der Vorderseite der Wirbel von der Schädelbasis bis zum Sakrum. Das hintere Längsband (Ligamentumlongitudinale posterius) beginnt am Clivus und endet

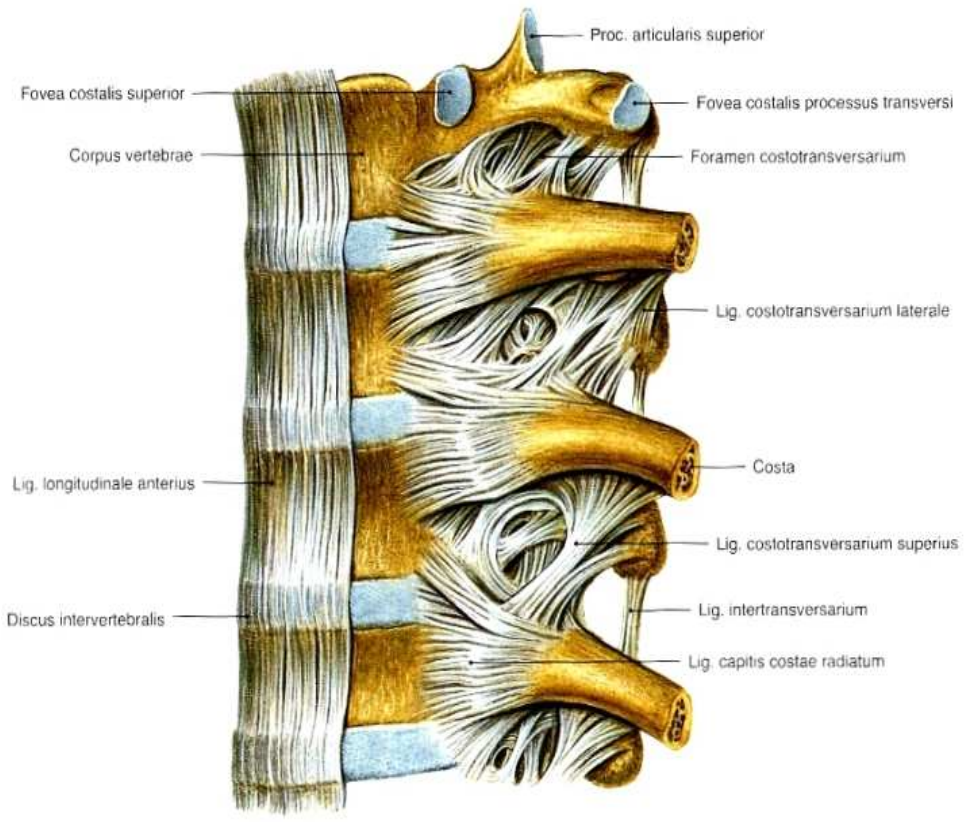

Abb. 1.2.5: Bänder der Wirbelsäule und Rippenwirbelverbindun gen; von links (SовотTA 2007, S. 279) im Canalis sacralis. Das hintere Band ist hauptsächlich mit den Bandscheiben verwachsen und liegt somit an der Vorderwand des Wirbelkanals (SCHIEBLER und KORF 2007). Des Weiteren verbindet das Ligamentum flavum, vorwiegend aus elastischen Fasern bestehend, die Unterseite der Lamina arcus vertebrae der oberen Wirbel mit der Oberkante der unteren Wirbel.

Die einzelnen Bänder sind:

- Ligamentum interspinale (zwischen den Dornfortsätzen) 
- Ligamentum supraspinale (zwischen den Spitzen der Dornfortsätze)

- Ligamentum intertransversarium (zwischen den Querfortsätzen).

Schließlich werden die Kapseln der Wirbelbogengelenke durch straffe Bandzüge, einen vorderen und einen hinteren, verstärkt. Alle Bänder gemeinsam führen zu einer stabilen Verbindung der Wirbel, die die Wirbelsäule mechanisch hoch belastbar macht (KAPANDJI 1985).

\subsubsection{Wirbel-Rippengelenke ( Artikulationes costovertebralis)}

Jede Rippe steht mit der Wirbelsäule in doppelter Verbindung, der Rippenkopf (Caput costae) mit dem Wirbelkörper und der Bandscheibe (Articulatio capitis costae) und der Rippenhals (Collum costae) mit dem Prozessus transversus (Articulatio costotransversaria). In einer Lateralansicht (Abb. 1.2.6) sieht man nur die Gelenkflächen des kostovertebralen

Gelenkes an der Wirbelsäule. Die

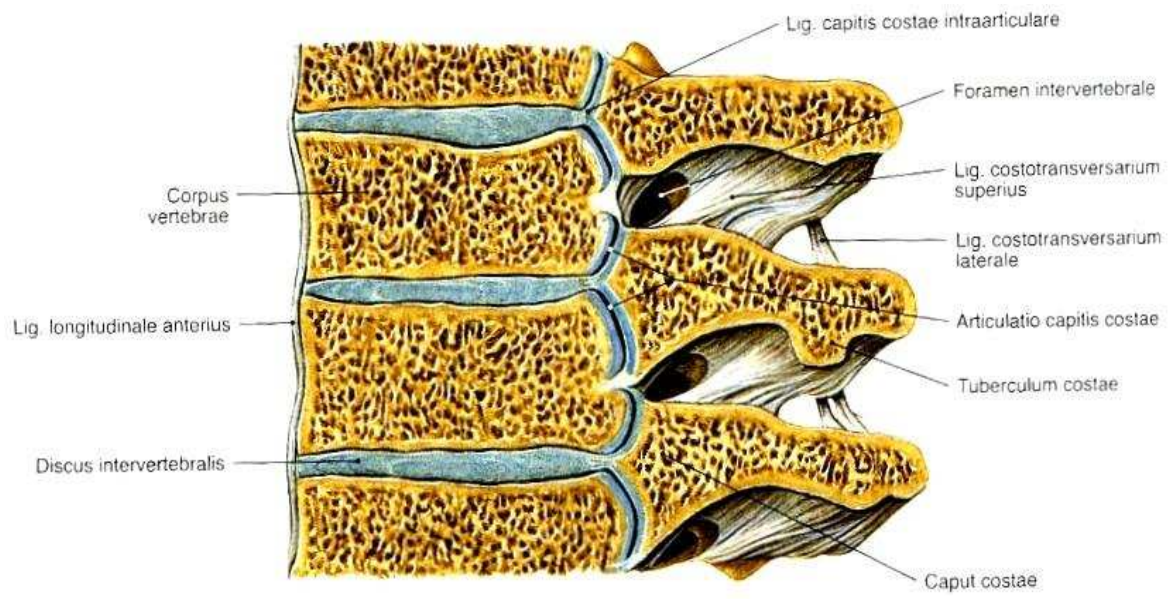

Articulatio capitis

costae (2. bis 10. Abb. 1.2.6: Rippenwirbelverbindungen; Articulationes costovertebrales von links ventral (SоBOTTA 2007, S. 280)

Rippe) ist ein ech-

tes Gelenk. Die korrespondierten Facetten des Caput costae sind leicht konvex und stehen abgewinkelt zueinander, so passen sie sich der Gelenkpfanne an (KAPANDJI 1985). Der Rippenkopf artikuliert mit den Fovea costales benachbarter Wirbel und der zugehörigen Bandscheibe, von der auch das Lig. capitis costae intraarticulare zum Rippenkopf zieht und das Gelenk in zwei Kammern teilt (Ausnahme 1., 11., 12. Rippe). Die Gelenkkapsel wird vorn durch das Lig. capitis costae radiatum verstärkt (WALDEYER und MAYET 2002).

In der Articulatio costotransversaria artikulieren zwei ovale Gelenkflächen miteinander. Die eine liegt am Tuberculum costae, die andere an der Spitze des 
Querfortsatzes. Das Gelenk wird von einer Bindegewebskapsel umschlossen, die von drei Ligamenta costatransversaria verstärkt wird (KAPANDJI 1985):

- Lig. costotransversarium laterale

- Lig. costotransversarium

- Lig. costotransversarium superius.

Durch Drehbewegung in den Kostovertebralgelenken können die ventralen Rippenanteile nach oben oder nach unten schwenken, was zur Erweiterung, bzw. zur Verengung des gesamten Thoraxraumes in sagittaler, vertikaler und transversaler Richtung führt (SCHIEBLER und KORF 2007).

\subsubsection{Muskelsysteme}

Alle Bewegungen der Brustwirbelsäule werden von einer Vielzahl von Muskel kontrolliert. Diese können Kräfte in unterschiedliche Richtungen ausüben, indem sie an den knöchernen Strukturen direkt angreifen.

\subsubsection{Rückenmuskulatur}

Die dorsale Rückenmuskulatur besteht aus Muskeln unterschiedlicher Herkunft. Nach ihrer Entwicklung kann man eine primäre, tiefe Muskelgruppe, die sich ortsständig entwickelt, auch autochthone Rückenmuskulatur genannt, und eine sekundäre, oberflächlich gelegene und von ventral eingewanderte Rückenmuskulatur unterscheiden. Die tiefe Schicht der autochthonen Muskulatur liegt unmittelbar der Wirbelsäule auf.

Die autochthone Rückenmuskulatur bildet in ihrer Gesamtheit zwei dicke Stränge, die rechts und links der Dornfortsätze eingebettet sind. Sie werden jeweils in einen medialen und einen lateralen Trakt eingeteilt. Sie sind an allen Bewegungen der Wirbelsäule (Flexion, Extension und Lateralflexion) beteiligt und werden in ihrer Gesamtheit als „Aufrichter der Wirbelsäule“, M. erector spinae, bezeichnet (SCHÜNKE 2000).

Auf die Beschreibung der einzelnen paravertebralen Muskeln wird in dieser Arbeit verzichtet. Jedoch geben die folgenden Tabellen eine Auskunft über die bestehenden funktionellen Systeme der Muskelgruppen. 
Medialer Trakt

\begin{tabular}{|c|c|}
\hline System & Funktion \\
\hline $\begin{array}{l}\text { Spinales System: } \\
\begin{array}{l}\text { - } \\
\text { - Mm. Interspinales } \\
\text { - M. spinalis }\end{array}\end{array}$ & $\begin{array}{l}\text { Dorsalextension } \\
\text { Lateralflexion } \\
\text { (HWS, BWS) }\end{array}$ \\
\hline $\begin{array}{l}\text { Transversospinales System: } \\
\begin{array}{l}\text { - } \\
\text { - }\end{array} \text { Mm. multifidus } \\
\text { - } \quad \text { M. semispinalis }\end{array}$ & $\begin{array}{l}\text { Dorsalextension } \\
\text { Lateralflexion } \\
\text { Rotation zur kontralateralen Seite } \\
\text { (Kopf, HWS, BWS) }\end{array}$ \\
\hline
\end{tabular}

Tab. 1.1: (SChebleR und Korf 2007, SchüNKE 2000, WALdeYer und MAYET 2002)

Lateraler Trakt

\begin{tabular}{|c|c|}
\hline System & Funktion \\
\hline $\begin{array}{l}\text { Sakrospinales System: } \\
\begin{array}{l}\text { - } \\
\text { M. iliocostalis } \\
\text { - } \quad \text { M. longissimus }\end{array}\end{array}$ & $\begin{array}{l}\text { Dorsalextension } \\
\text { Lateralflexion } \\
\text { Exspiration + Inspiration }\end{array}$ \\
\hline $\begin{array}{l}\text { Spinotransversales System: } \\
\text { - } \quad \text { M. splenius }\end{array}$ & $\begin{array}{l}\text { Dorsalextension } \\
\text { Lateralflexion } \\
\text { Rotation zur ipsilateralen Seite } \\
\text { (Kopf, HWS) }\end{array}$ \\
\hline $\begin{array}{l}\text { Intertransversales System: } \\
\begin{array}{c}\text { - } \\
\text { - }\end{array} \text { Mm. intertransversarii } \\
\end{array}$ & $\begin{array}{l}\text { Dorsalextension } \\
\text { Lateralflexion } \\
\text { Rotation zur kontralateralen Seite } \\
\text { (HWS, LWS) }\end{array}$ \\
\hline
\end{tabular}

Tab. 1.2: (SCHEBlER und KoRF 2007, SCHÜNKE 2000, WALDEYER und MAYET 2002)

Alle paravertebralen Muskeln wirken direkt oder indirekt auf die Wirbelbogengelenke, die sie entweder durch einen ausgewogenen Tonus in Ruhelage halten und damit die Wirbelsäule stabilisieren, oder bei einer Tonusänderung (Kontraktion) bewegen (SCHIEBLER und KORF 2007).

Einen weiteren Stabilisierungseffekt hat die oberflächliche Rückenmuskulatur.

Diese nach dorsal gewanderten Muskeln verknüpfen die Wirbelsäule sowohl mit dem Thorax, deswegen spinokostale Muskeln genannt, als auch mit dem Schul- 
tergürtel, deswegen als spinokapsuläre bzw. spinohumerale Muskeln bezeichnet (SCHIEBLER und KORF 2007).

Nichtautochthone Muskeln

\begin{tabular}{|c|l|}
\hline Muskulatur & Funktion \\
\hline Spinokostale: & $\begin{array}{l}\text { Rippenatmung } \\
\text { Inspiration } \\
\text { - M. seratus superior }\end{array}$ \\
- M. seratus inferior & Exspiration \\
\hline Spinohumerale: & $\begin{array}{l}\text { Brustatmung / Schulterblattheber } \\
\text { Inspiration } \\
\text { - M. latissimus dorsi }\end{array}$ \\
- M. rhomboideus major und minor & Exspiration \\
$-\quad$ M. trapezius & \\
\hline
\end{tabular}

Tab. 1.3: (SchebleR und KORF 2007, SchÜNKE 2000, WALDAYER und MAYET 2002)

Das komplette Muskelsystem funktioniert wie eine geschlossene Einheit und kann somit nicht separat betrachten werden. Die Wirkung unterschiedlicher Muskeln summiert sich zur Muskelkraft, die in dieser Arbeit durch eine Kraftschraube (s. Biomechanik) simuliert wird.

Auf dieser Basis kann der Einstieg in die Biomechanik und Kinematik der Wirbelsäule erfolgen. 


\subsection{Mathematische und physikalische Grundlagen}

\subsubsection{Vektorrechnung}

\subsubsection{Vektoren}

Die Größen, die eine Richtung im Raum haben, bezeichnet man in der Mathematik als Vektoren; sie sind durch Betrag (Länge) und Richtung im Raum definiert. Die Skalare dagegen sind ungerichtete Größen. Ein Vektor $\underline{r}$ ist durch ein Zahlentripel $\left(r_{x}, r_{y}, r_{z}\right)$ beschrieben. $r_{x}, r_{y}$ und $r_{z}$ sind die Komponenten des Vektors im vorgegebenen Koordinatensystem (Abb 1.3.1).

Die Länge des Vektors ergibt sich aus dem Betrag

$$
\underline{\mid r} \mid=r=\sqrt{\left(r_{x}^{2}+r_{y}^{2}+r_{z}^{2}\right)}
$$

Der Vektor $\underline{a}$ ist das Produkt aus Betrag a mal dem Einheitsvektor $\underline{\mathrm{e}}=\left(e_{\mathrm{x}}, \mathrm{e}_{\mathrm{y}}, \mathrm{e}_{\mathrm{z}}\right)$. Der normierte Richtungsvektor $\underline{\mathrm{e}}$ hat den Betrag lel $=1$ (Einheitsvektor): $\quad \underline{r}=r \cdot\left(e_{x}, e_{y}, e_{z}\right)$

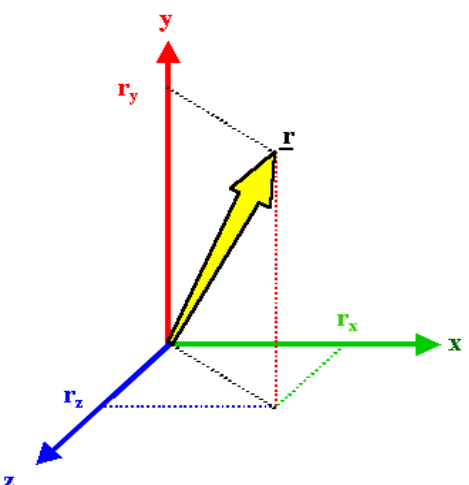

Abb. 1.3.1: Vektor (Pfeil)

Physikalisch sind verschiedene Vektortypen zu unterscheiden:

Ein linienflüchtiger Vektor (die Kraft F) kann ohne Änderung der Wirkung nur entlang seiner Wirkungslinie verschoben werden.

Dagegen ist das Drehmoment ein freier Vektor, er kann ohne Änderung der Wirkung beliebig im Raum verschoben werden.

\subsubsection{Skalarprodukt der Vektorrechnung}

Skalare sind absolute Größen, die unabhängig von einer Orientierung sind. So ist zum Beispiel die physikalische Arbeit das Skalarprodukt (Abb. 1.3.2) aus dem Kraft-

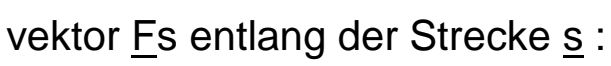

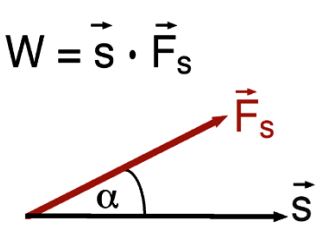

$$
\mathrm{W}=\underline{\mathrm{F}} \cdot \underline{\mathrm{s}} \Rightarrow \mathrm{W}=|\underline{\mathrm{F}}| \cdot|\underline{\mathrm{s}}| \cdot \cos \alpha
$$

Das Produkt zweier Skalare kann auch Null sein, wenn die Vektoren parallel $(\alpha=0)$ zu einander stehen. 


\subsubsection{Kreuzprodukt der Vektorrechnung}

Die Vektoren $\underline{F}$ und $\underline{L}$ schneiden sich in $P$ mit dem Winkel $\alpha$ (Abb. 1.3.3).

Das Vektorprodukt $\underline{\mathrm{L}} \times \underline{\mathrm{F}}$ bestimmt einen dritten Vektor $\underline{I}$, der zur $\underline{L}$ und $\underline{E}$ senkrecht ist. Zum Beispiel der Drehmomentsvektor I berechnet sich aus dem Vektorprodukt des Hebelarms $\underline{L}$ und der Kraft $\underline{F}$.

Die Vektorlänge errechnet sich aus:

$$
|\underline{I T}=| \underline{I}|\cdot| \underline{F} \mid \cdot \sin \alpha
$$

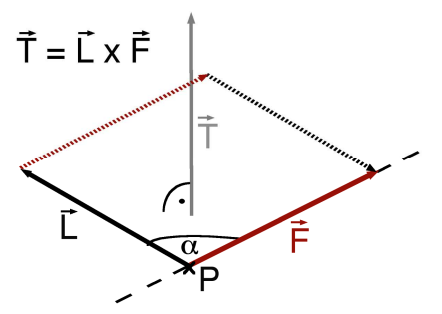

Abb. 1.3.3:

Kreuzprodukt

\subsubsection{Vektorielle Darstellungen von Geraden und Ebenen}

Die Lage einer Geraden im Raum lässt sich durch zwei Punkte mit den Ortsvektoren $\underline{x}_{0}$ und $\underline{x}_{1}$ definieren $g$ : (Abb. 1.3.4). Jeder Punkt auf dieser Geraden lässt sich wie gefolgt definieren:

$$
\mathrm{g}:=\underline{\mathrm{x}} 0 \bullet+\lambda \bullet \underline{\mathrm{e}} ;
$$

Geraden können zueinander parallel, antiparallel oder

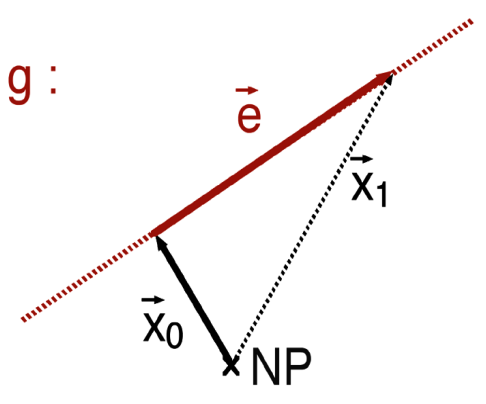
windschief liegen oder sich in einem Punkt schneiden.

Ebenen kann man ebenfalls durch einen Ortsvektor $\underline{x}_{0}$ und zwei Richtungsvektoren $\mathrm{a}$ und $\mathrm{b}\left(\mathrm{a} \cap \mathrm{b}\right.$ in $\mathrm{P}_{0}$ ) definieren und damit jeden Punkt der Ebene exakt bestimmen:

$$
\underline{\mathrm{e}}:=\underline{\mathrm{x}}_{0} \bullet+\lambda \bullet \underline{\mathrm{a}}+\delta \bullet \underline{\mathrm{b}}
$$

Für den Fall $\underline{\mathrm{x}}_{0} \perp \underline{\mathrm{a}}$ und $\underline{\mathrm{x}}_{0} \perp \underline{\mathrm{b}}$ gilt: $\left|\underline{\mathrm{x}}_{0}\right|$ ist der kürzeste Abstand zum Nullpunkt.

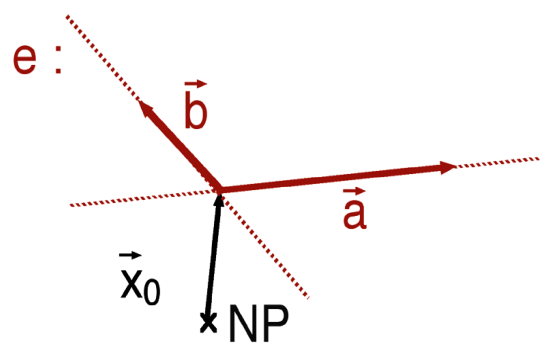

Abb. 1.3.4: Geraden und Ebenengleichung 


\subsubsection{Beschreibung der Bewegungsstruktur}

Um die räumliche Bewegung eines starren Körpers beschreiben zu können, kann man die Positionsveränderung dreier Punkte mit drei Koordinaten $(x, y, z)$ verfolgen. $\mathrm{Da}$ die Seitenlängen $a, b$ und $c$ (Dreieck in Abb. 1.3.5) eines starren Körpers konstant sind, sind von den 9 Koordinaten nur sechs variabel. Das entspricht sechs Freiheitsgraden der Bewegung. Ein Körper bewegt sich von seiner Neutralposition „0“ in eine beliebige Position "1“ (Abb. 1.3.5).
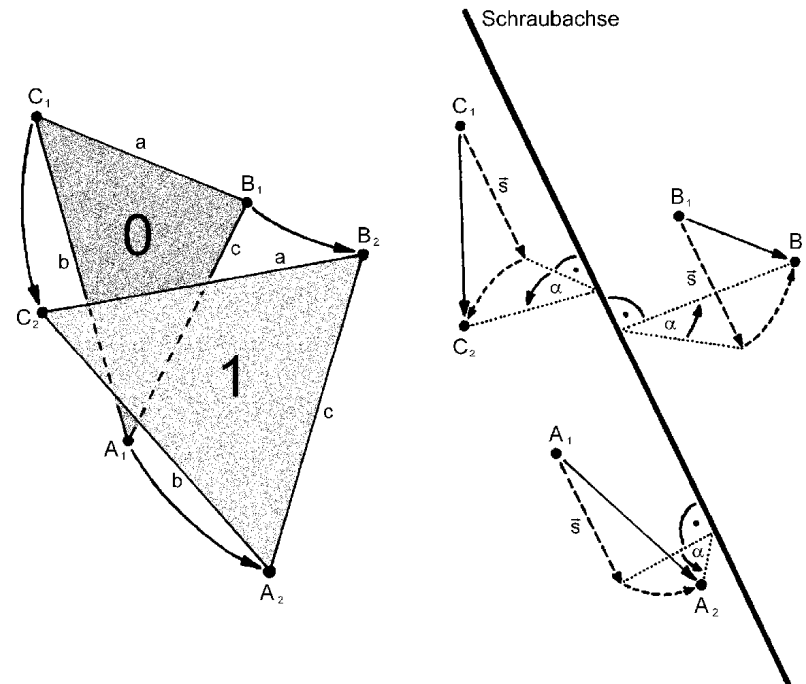

Abb. 1.3.5: Räumliche Bewegung eines starren Körpers aus der Position 0 nach 1 (links). Das rechte Bild zeigt die Bewegung auf eine Verschraubung reduziert.

Nach dem Satz von Chasles (TEICHMANN 1973) lässt sich jede diese Bewegung durch eine Schraubenbewegung oder Verschraubung beschreiben, das heißt durch eine Rotation des Körpers $(\angle \underline{\alpha})$ um eine räumliche Gerade, die Schraubachse genannt wird;

(SA) $\underline{r}(\alpha, \lambda)=\underline{x}_{0} \bullet(\alpha)+\lambda \bullet \underline{e}(\alpha)$

und gleichzeitig durch eine Translation mit $\underline{s}=\lambda \bullet \underline{e}(\alpha)$ entlang der Schraubachse. Der Quotient $|\underline{\underline{s}}| /|\underline{\alpha}|$ entspricht der Schraubsteigung $\tau$.

Ist im mathematisch-rechtshändigen Koordinatensystem (Abb. 1.3.6) eine Schraubsteigung $\tau>0$, so spricht man von eine Rechtsschraubung, bei $\tau<0$ ist es eine Linksschraubung. Die Schraubsteigung $\tau=0$ stellt eine reine Rotation in einer Ebene dar. Die Rotation um eine unendlich entfernte Schraubachse wäre als eine reine Translation zu verstehen.

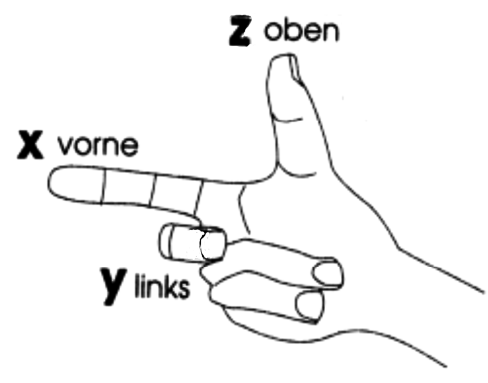

Abb. 1.3.6: „Rechte Handregel“ (modifiziert nach KRISMER 1996, S.16). 
Dem zur Folge ist jede Bewegung eines starren Körpers im Raum mit Hilfe von nur sechs Parametern beschreibbar:

1. Dem Ortsvektor der Schraubachse oder einem Schnittpunkt mit einer definierten Ebene xy (2 Freiheitsgrade).

2. Dem normierten Richtungsvektor der Schraubachse $\underline{e}(\alpha)$ als Funktion des Drehwinkels lel $=\sqrt{\left(e_{X}^{2}+e_{Y}^{2}+e_{Z}^{2}\right)}=1$ ( 2 Freiheitsgrade).

3. Dem Rotationswinkel $\underline{\alpha}$ (1 Freiheitsgrad).

4. Dem Versatz $|\underline{s}|$ entlang der Schraubachse und der daraus errechneten Schraubsteigung $\tau(\alpha)$ als Funktion des Drehwinkels (1 Freiheitsgrad).

Diese Darstellung beschreibt nur den einfachsten Übergang vom Anfangs- zum Endpunkt, wie sie auch von PANJABI ET AL. (1986) benutzt wurde. Eine Aussage über den tatsächlich zurückgelegten Weg kann nicht erfolgen. Tatsächlich kann der Körper auf den Umwegen jede mögliche Position annehmen (Abb. 1.3.7). Nur wenn eine Positionsänderung von „,0“ auf „"“ differentiell klein ist, approximiert die Elementarschraubung die Laufbahn der Bewegung. Es resultiert eine differentielle (momentane) Schraubachse $\operatorname{dr}(\alpha)$ mit

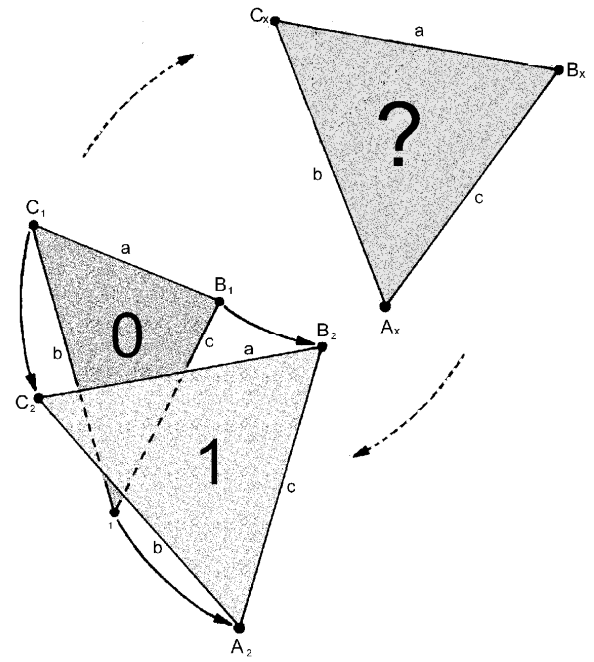

Abb. 1.3.7: Eine differentielle Betrachtung der Bewegung von 0 nach 1 ist notwendig, um mögliche „Umwege“ über „?" aufzulösen. dem Drehwinkel $d \alpha$ und der differentiellen Schraubsteigung $\tau(\alpha)$. Vom „Abschroten“ spricht man bei einer Bewegung entlang der Drehachse.

Im Allgemeinen ändert sich die Position der momentanen Schraubachsen mit der Bewegung. Die Funktion aller Schnittpunkte (dR) der momentanen Schraubachsen mit einer definierten Ebene bezeichnet man als Rastpolkurve. Die Summe aller Schraubachsen (Geradenschar) bezeichnet man als Regelfläche. 


\subsubsection{Kraftsysteme}

Aus der Sicht der klassischen Mechanik kann man das Kraftsystem, die Gesamtheit aller auf ein Bewegungssegment einwirkenden Kräfte, auf zwei Komponenten reduzieren: eine Kraft $\underline{F}$ und ein kräftefreies Drehmoment $\underline{T}$.

\subsubsection{Kräfte}

Kräfte sind vektorielle Größen. Sie sind durch ihren Betrag, Richtung und Orientierung bestimmt. Aufgrund ihrer Eigenschaften kann man einen Kraftvektor nur entlang seiner Wirkungslinie verschieben (polarer Vektor).

Eine Kraft, die im Schwerpunkt ansetzt, bewirkt eine reine Translation.

Analog zum Schwerpunkt, spricht man bei einem elastisch befestigten Körper von dem Widerstandszentrum W. Bei hinreichend homogener Elastizität kann es dem Symmetriemittelpunkt entsprechen.

Kräfte lassen sich nach den Gesetzen der Vektoraddition, -subtraktion und der skalaren Multiplikation beliebig durch andere Kräfte darstellen.

\subsubsection{Drehmomente}

Eine vom Widerstandszentrum um Vektor $\underline{\mathrm{L}}$ versetzte Kraft $\underline{F}$ erzeugt ein Drehmoment $\underline{I}=\underline{L} \times \underline{F}$ (Abb. 1.3.8 A). Ein reines Drehmoment bewirkt eine Rotation. Der Drehmomentvektor $\underline{I}$ steht senkrecht zu der von $\underline{L}$ und $\underline{F}$ aufgespannten Ebene. Der Betrag des Drehmoments bestimmt sich aus $|\underline{I}|=|\underline{L}| \bullet|\underline{F}| \bullet$ sin $\alpha$. Für III ist hierbei nun der zum Hebelarm senkrechte Kraftanteil entscheidend. Die Fläche des von $\underline{L}$ und $\underline{F}$ aufgespannten Parallelogramms entspricht geometrisch der Größe vom Drehmoment $(\mathrm{T})$.

Ein reines Drehmoment wird durch ein Kräftepaar ( $\underline{F},-\underline{F})$ erzeugt, also durch eine Kraft und

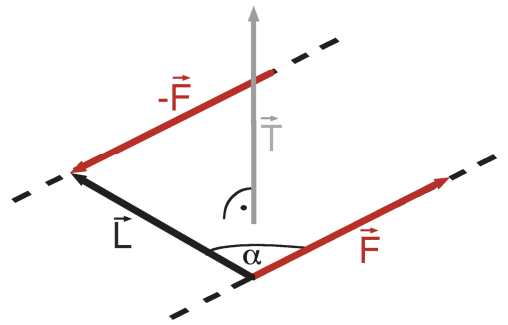

Abb. 1.3.8.A:Ein Kräftepaar erzeugt ein Drehmoment

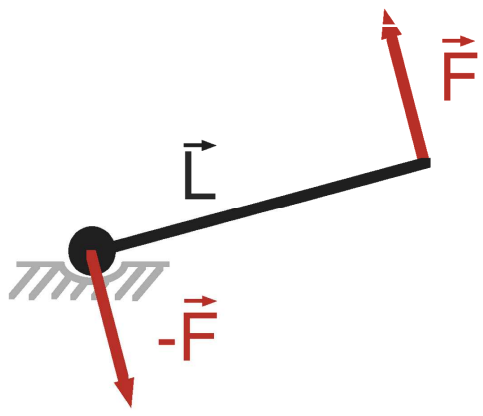

Abb. 1.3.8.B: Eine Kraft $\underline{F}$ erzeugt eine Gegenkraft - $\underline{F}$. 
eine Gegenkraft (Abb.1.3.8 B, S. 24), man spricht auch von einer sogenannten Lagerkraft.

Solange alle Kräftepaare (Vektoren) in parallelen Ebenen liegen und das gleiche Produkt aus $\underline{\underline{L}} \cdot \underline{\mathrm{F}}$ haben, resultieren sie in demselben Drehmoment $\underline{\mathrm{T}}$.

\subsubsection{Kraftschraube}

Als Kraftsystem bezeichnet man die Gesamtheit aller an einem starren Körper angreifende Kräfte und Drehmomente. Räumliche Kraftsysteme haben, wie auch die Lage des Körpers, sechs Freiheitsgrade. Schneiden sich die Wirkungslinien der Vektoren in einem Angriffspunkt, so lässt sich eine resultierende Kraft aus der vektoriellen Summe aller Kräfte (Vektoren) bestimmen.

Die translatorischen Kräfte wirken in drei Raumrichtungen (3 Freiheitsgraden). Sie lassen sich auf eine Kraft reduzieren, die durch den Schwerpunkt des Körpers geht. Analog verfährt man mit den Drehmomenten um die drei Raumrichtungen $(x ; y ; z)$, so dass man eine resultierende Kraft erhält.

Alle Komponente des Kraftsystems lassen sich zu einer resultierende Kraft $\underline{F}$ $\left(F_{X}, F_{Y}, F_{Z}\right)$ mit einer Kraftwirkungslinie durch den Ursprung des Koordinatensystems und einem Drehmoment $I\left(T_{X}, T_{Y}, T_{Z}\right)$ zusammenfassen. Wenn man die Kraftwirkungslinie außerhalb des Koordinatenursprungs legt und parallel verschiebt, lässt sich dieses Kraftsystem auf ein äquivalentes Kraftsystem mit ausgezeichneter Kraftangriffslinie reduzieren (WoLF 1931). Die axiale Kraft $\underline{z} z$ und das Drehmoment $I$ als Kräftepaar in einer Ebene weisen dann die gleiche Richtung (nicht notwendigerweise die gleiche Orientierung) auf. Das entstandene Kraftsystem hat eine äquivalente Wirkung auf den starren Körper. Diese beiden vektoriellen Größen beschreiben eine Kraftschraube mit $\underline{I}\left(T_{X}, T_{Y}, T_{Z}\right)$ und $\underline{E}\left(F_{X}, F_{Y}, F_{Z}\right)$.

Die Kraftschraube wird durch 6 Parameter (Freiheitsgrade) definiert:

- den Fußpunkt $\left(\mathrm{x}_{\mathrm{F}}, \mathrm{y}_{\mathrm{F}}\right)$ des Kraftvektors in einer definierten Ebene (zwei Freiheitsgrade)

- den normierten Richtungsvektor $\underline{e}_{F}$ der Kraftwirkungslinie (zwei Freiheitsgrade)

- den Betrag des zur Kraftwirkungslinie parallelen Drehmoments 
(ein Freiheitsgrad)

- den Betrag der resultierenden Kraft (ein Freiheitsgrad).

In einem ebenen Kraftsystem kann keine Kraftschraube entstehen, da sie sich entweder auf eine Einzelkraft oder ein Kräftepaar (Drehmoment) reduzieren lässt. Man kann ein und dasselbe Drehmoment durch beliebig viele Kräftepaare erzeugen. Das gilt auch für eine resultierende Kraft F, die nach den Gesetzen der Vektoraddition aus beliebig vielen Einzelkräften reduziert werden kann.

Daraus folgt, dass es unendlich viele verschiedene Kräftekombinationen gibt, um eine bestimmte Kraftschraube zu erzeugen. Zwei Kraftsysteme mit unterschiedlichen Kräftekonstellationen, aber derselben Wirkung auf einen starren Körper, bezeichnet man als „Äquivalente Kraftsysteme“ (NÄGERL 1990).

Bezogen auf den menschlichen Körper wird ersichtlich, dass das Zusammenwirken einer Vielzahl von Muskeln biophysikalisch durch eine Kraftschraube beschrieben werden kann. Es ist aber nicht möglich, bei Kenntnis der resultierenden Kraftschraube, eine Aussage über diese Kraftschraube erzeugten Muskelkräfte zu treffen.

Im einfachsten Fall realisiert sich eine Kraftschraube aus einer axialen Vorlast $\underline{F}_{Z}\left(0,0, F_{Z}\right)$ und einem parallelen Drehmoment $\underline{I}_{z}\left(0,0, T_{z}\right)$, wie es auch in dieser Arbeit verwendet wurde (Kap. 2.1).

Abb. 1.3.9: Äquivalente Kraftsysteme: Die Einzelkraft $\underline{F}(A)$ zeigt dieselbe Wirkung wie die Einzelkraft $\underline{F}$ durch das Widerstandszentrum $W$ zusammen mit dem Kräftepaar ( $\underline{F},-\underline{F})$ mit dem Abstand a von W (B). Das Kräftepaar lässt sich auch durch ein Drehmoment $\underline{I}=\underline{a} \times \underline{F}(\mathrm{C})$ darstellen. Das Drehmoment kann auch durch unendlich viele andere Kräftepaare erzeugt werden. Der Übertragungspunkt von I auf den Körper ist dabei gleichgültig.

A
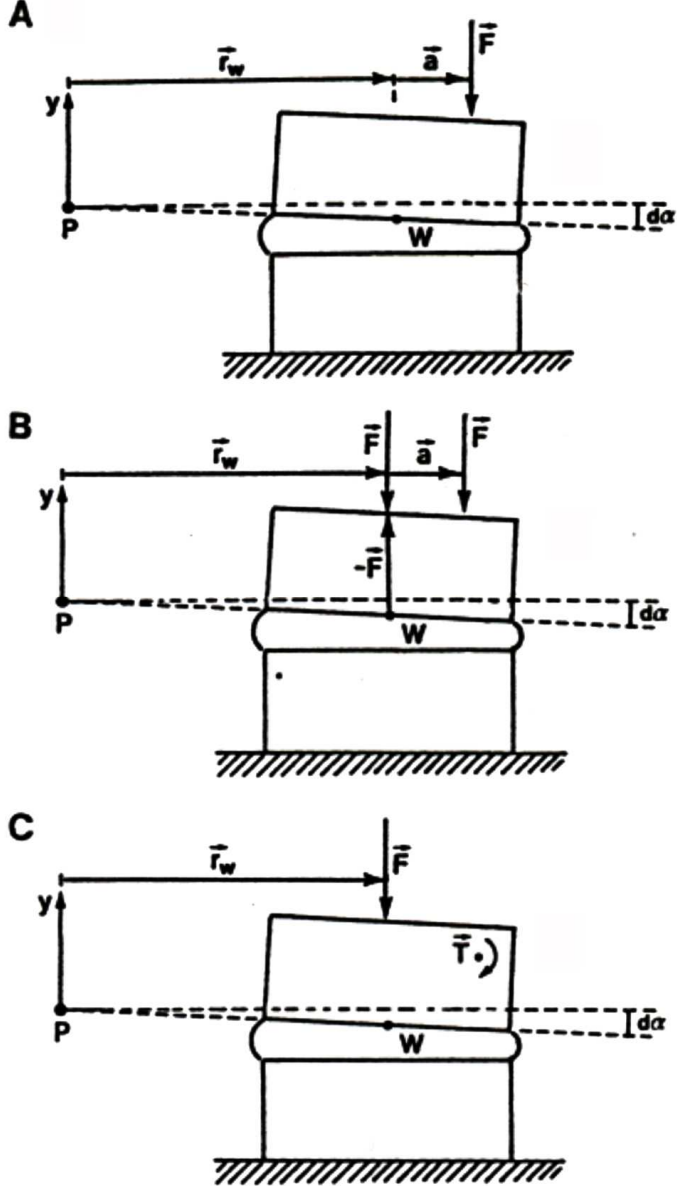


\subsubsection{Viskoelastizität}

Die biologischen Strukturen wie Bandscheiben und Bänder sind keine idealen Hook'schen Federn. Sie sind nicht in der Lage die mechanische Energie verlustfrei zu speichern. Die Auslenkung $\mathrm{s}$ ist aber trotzdem zur aufgebrachten Kraft $\underline{\mathrm{F}}$ annähernd proportional. Die Verformbarkeit der biologischen Strukturen führt zu Dämpfungseffekten, wodurch bei abrupter Änderung der Kraft $\underline{F}$ die Auslenkung erst zeitlich versetzt in der neuen Position endet. Bei periodischer sinusförmiger Veränderung der Krafteinwirkung folgt die Auslenkung phasenverschoben. Die Auslenkungs-Kraft-Kurve $\underline{\mathbf{s}}(\underline{F})$ bzw. die Drehwinkel-Drehmoment-Kurve $\alpha(T)$ erfährt eine Hysterese. 


\subsection{Biomechanik komplexer Gelenke}

\subsubsection{Bänder, Menisken}

Meniski haben keinen qualitativen Einfluss auf die Gelenkführung. Sie sind mit den artikulierenden Flächen nicht fest verbunden. Die Meniski dienen der Verkleinerung der Gelenkräume. Durch sie wird das benötigte Synovialvolumen verringert und somit die Gelenkschmierung gesichert (NäGERL 1990). Die Meniski in Verbindung mit der Gelenkkapsel erhalten zahlreiche Mechanorezeptoren, vor allem in ihrer Aufhängung (BLÜMLE 1997), wie zum Beispiel die meniskoiden Falten an den Gelenkkapseln der Artt. zygapophysiales (ZUKSCHWERDT ET AL. 1960, MCLAIN 1994, LUSCHKA 1856). Die Mechanorezeptoren registrieren die Relativlage zwischen oberen und unteren Wirbelkörpern und erleichtern somit die neuromuskuläre Steuerung.

Bänder sind auf Zug belastbar. Sie nehmen zum Beispiel unphysiologische Bewegungen auf und verhindern so die Luxationen.

Das Bewegungsausmaß der Wirbelsäule wird durch die anspannenden Bänder begrenzt. Sie besitzen im Hinblick auf die Bewegungsstruktur der Artt. zygapophysiales keine Führungsqualität (KALSCHEUER 2001). So kann man sagen, dass die Bänder und Menisken durch ihre Elastizität bzw. Steifigkeit lediglich die Quantität der Bewegung modulieren, während die Gelenkoberflächen bei Kraftschluss das Bewegungsschema definieren (NäGERL ET AL.1995).

\subsubsection{Synarthrosen}

Eine reine Synarthrose ist zum Beispiel eine Bandscheibe. Folgend wird hier die Theorie der Mechanik von Synarthrosen anhand des Systems WirbelkörperBandscheibe-Wirbelkörper beschrieben. Es entspricht zwei starren Körpern, die durch eine viskoelastische Scheibe verbunden sind. Die Wirbelbogengelenke sind entfernt.

Für die folgenden Betrachtungen wird die Bandscheibe vereinfacht als kreisrund mit dem Radius d, der Fläche $A=\pi \bullet d^{2}$ und der Dicke $h$ angenommen (Abb. 1.4.1). 
Sie sei zunächst homogen-elastisch mit dem Elastizitätsmodul E und dem Schubmodul G. Es gilt das Hook'sche Gesetz.

\title{
1.4.2.1 Definition des Widerstandszentrums
}

Im Allgemeinen wird der Massenschwerpunkt eines Körpers als Widerstandszentrum gesehen. Das Widerstandszentrum W eines elastisch befestigten Körpers existiert als Punkt nur bei hinreichend großer Symmetrie des Körpers (DATHE 2001). Bei hoher homogener Elastizität liegt es im Symmetriemittelpunkt. Das Widerstandszentrum ist der Raumpunkt eines mechanischen Systems, in dem Translation und Rotation entkoppeln. So führen die Kräfte, die im Widerstandszentrum ansetzen, zu einer reinen Translation und applizierte Drehmomente zu einer reinen Rotation mit einer Achse, welche durch das Widerstandszentrum $(\mathrm{W})$ verläuft.

Der obere Wirbelkörper ist beweglich, der untere wird als ortsfest angesehen. Das Widerstandszentrum W des Segments wird durch die vertikale Sym-

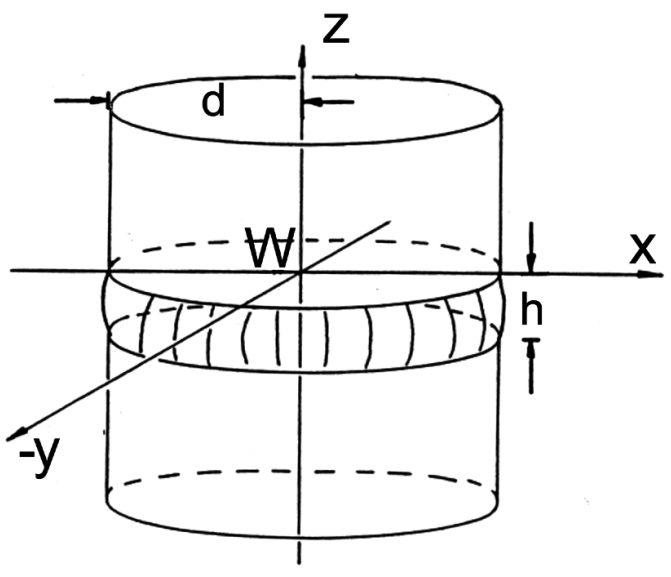
metrieachse z markiert (NÄGERL 1990). Senkrecht dazu schneiden die sagittale Symmetrieachse $x$ und transversale Achse y (Abb.1.4.1).

\begin{abstract}
Abb. 1.4.1: Modell einer Synarthrose Mit Zwischenscheibe Dicke h und Durchmesser d. Das Widerstandszentrum liegt in der Symmetrieachse.
\end{abstract}

\subsubsection{Kräfte durch das Widerstandszentrum}

Bei einer Krafteinwirkung im Widerstandszentrum kommt es zu einer Parallelverschiebung in Richtung der Kraft, ohne Verkippung. Diese translatorischen Änderungen wurden als $\Delta \mathrm{X}, \Delta \mathrm{Y}$ und $\Delta \mathrm{Z}$ durch entsprechende $\mathrm{Kräfte} \mathrm{F}_{\mathrm{X}}, \mathrm{F}_{\mathrm{Y}}, \mathrm{F}_{\mathrm{Z}}$ bezeichnet.

Bei einer senkrecht wirkenden Kraft $F_{Z}$ resultiert eine Dehnung bzw. Stauchung der elastischen Bandscheibe um $\pm \Delta Z$ mit dem Elastizitätsmodul $\mathrm{E}$. 
Nach der Formulierung des Hook'schen Gesetzes gilt: $F_{Z}=E \bullet \Delta z \bullet(A / h) \bullet \Delta z$.

Bei einem bekannten Körper gilt: $F_{Z}=D_{E} \bullet \Delta z$. Kräfte in Richtung der $x$ - und yAchse $F_{X}$ und $F_{Y}$ führen zu einer Abscherung und Verschiebung um $\Delta x$ oder $\Delta y$. Es gilt das Hook'sche Gesetz für Scherbeanspruchung mit dem Schubmodul G (Abb. 1.4.2 A):

$F_{X}=G \bullet(A / h) \bullet \Delta x \wedge F_{Y}=G \bullet(A / h) \bullet \Delta y$ oder $F_{X}=D_{S} \bullet \Delta x \wedge F_{Y}=D_{S} \bullet \Delta y$ für einen bekannten Körper
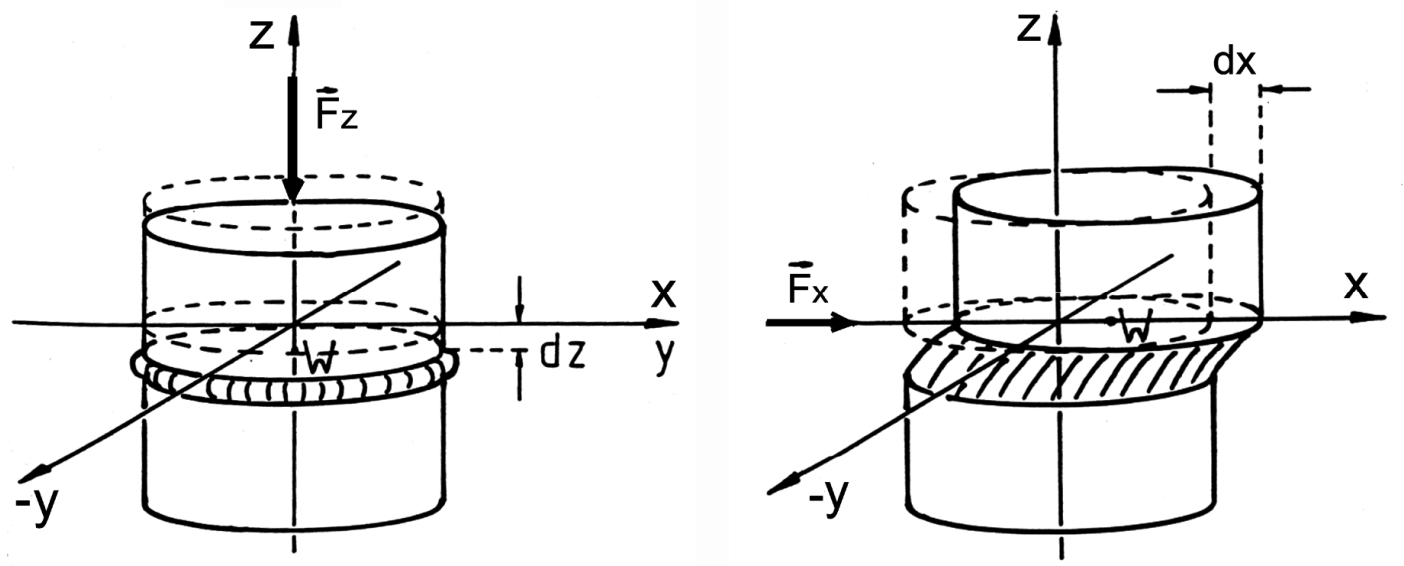

Abb. 1.4.2 A: Kraftwirkungslinie durch das Widerstandszentrum $\mathrm{W}$ am Beispiel einer axialen Kraft $F_{Z}$ und einer Scherkraft $F_{X} \cdot F_{Y}$ hätte eine entsprechende Wirkung.

Da das Elastizitätsmodul eine ganz andere Größe hat als das Schermodul, kommt

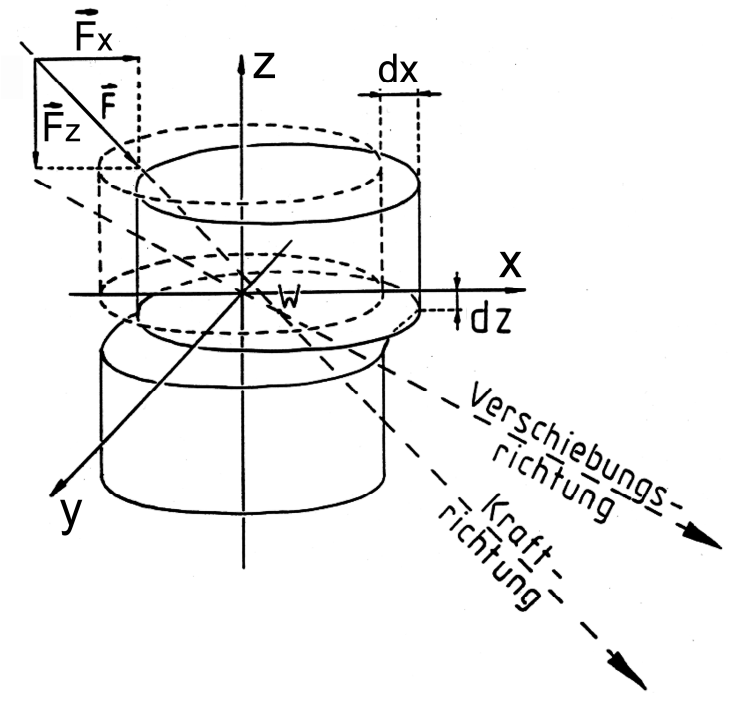

Abb. 1.4.2.B: Wirkung einer Kraft $\underline{F}(F x, 0, F z)$ nun im Fall einer Kraft $\underline{E}\left(F_{X}, 0, F_{Z}\right)$, die der Winkelhalbierenden der $\mathrm{x}$ - und $\mathrm{z}$ Achse folgt, Folgendes heraus: Es resultiert eine Scherung um $\Delta \mathrm{x}$ und eine Stauchung der Bandscheibe um $\Delta z$. Die Translation in $x-, y$-Achse wird vom Schubmodul bestimmt, die axiale Stauchung entlang der $z$ Achse vom Elastizitätsmodul. Da die Größe des Schubmoduls zwei bis dreifach geringer ist als die des Elastizitätsmoduls, folgt der obere Wirbelkörper nicht der Kraftwirkungslinie. Es kommt zu einer proportional größeren 
Scherung. Der Verschiebungsvektor wird flacher als der Kraftwirkungsvektor (Abb. 1.4.2 B, S. 30).

\subsubsection{Reine Drehmomente und achsenparallele Kräfte}

Ein reines Drehmoment $T_{X}$ oder $T_{Y}$ führt zu einer Rotation $\alpha_{X}$ oder $\alpha_{Y}$ um eine Drehachse Py bzw. PX.

Mit Berücksichtigung des Elastizitätsmoduls $\mathrm{E}$ gilt (Abb. 1.4.3 A):

$$
\begin{aligned}
& T_{X}=E \bullet(\pi / 4) \bullet\left(d^{4} / h\right) \bullet \alpha_{X} \wedge T_{Y}=E \bullet(\pi / 4) \bullet\left(d^{4} / h\right) \bullet \alpha_{Y} \\
& \text { und } T_{X}=D_{B} \bullet \alpha_{X} \wedge T_{Y}=D_{B} \bullet \alpha_{Y} \text { für bekannte Größen. }
\end{aligned}
$$

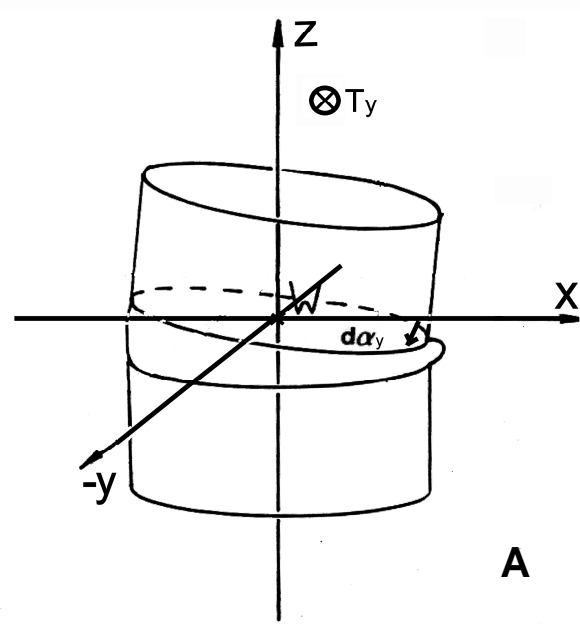

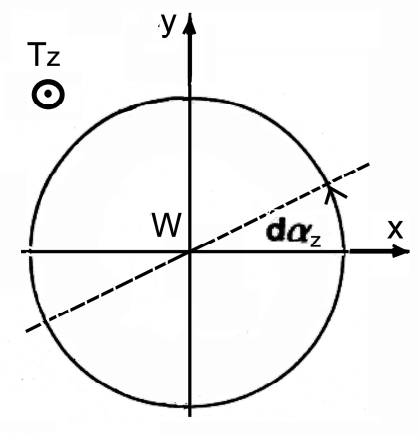

B
Abb. 1.4.3: Wirkung eines reinen Drehmoments: Das axiale Drehmoment in Richtung $y$-Achse $T_{y}(A)$ und Richtung z-Achse $\mathrm{Tz}(\mathbf{B})$

Bei der Verschiebung der Kraftwirkungslinie einer Kraft $F$ entlang der $x$ - oder $y$ Achse um ax oder ay parallel zur z-Achse resultiert eine Rotation um $\alpha_{Y}$ oder $\alpha_{X}$ mit dem Drehmoment $T_{Y}=F_{Z} \times a_{x}$ oder $T_{X}=F_{Z} \times a_{x}$ und der Wirbel verkippt. Die Rotationsachse $P$ liegt auf der gegenüberliegenden Seite des Widerstandszentrums von $\mathrm{F}_{Z}$ und steht senkrecht zum Abstand a der Kraftwirkungslinie.

Für ein Drehmoment in Richtung z-Achse $T_{Z}$ mit dem Abstand a $\left(T_{Z}=F_{Z} \times a\right)$ liegen die Verhältnisse ähnlich. Es kommt zu einer Rotation $\alpha_{z}$ um die vertikale Symmetrieachse $z$ in Abhängigkeit vom Schermodul G (Abb. 1.4.3 B).

Es gilt: $T_{z}=G \bullet(\pi / 2) \bullet\left(d^{4} / h\right) \bullet \alpha_{z}$. 
Für Synarthrosen gilt das Reziprozitätstheorem: $\sigma^{2}=a \cdot r_{w}=$ konst, welche besagt,

" je größer der Abstand a zwischen Widerstandszentrum $W$ und Kraftdurchstoßpunkt $A$, desto näher rückt die in Horizontalebene liegende Drehachse $P$ an das Widerstandszentrum $W$ heran (Abstand $r_{W}$ )“

(NÄGERL 1990, S. 69).

Es beschreibt mathematisch den Zusammenhang zwischen der wirkenden Kraft und der daraus resultierenden Bewegung. Steigt der Abstand a, so rückt die Drehachse $\mathrm{P}$ an das Widerstandszentrum $\mathrm{W}$ heran. Der Abstand $\mathrm{r}_{\mathrm{W}}$ wird kleiner. Für den Grenzfall $r_{w} \rightarrow 0$ kommt es zu einer reinen Rotation mit einer Drehachse im Widerstandszentrum. Wird der Abstand a kleiner, $a \rightarrow 0$, geht die Kraft durch W, und die Rotationsachse rückt ins Unendliche. Es kommt zur einen Translation. So kann die Zwischenwirbelscheibe als eine reine Synarthrose die Normalkräfte und entsprechende Drehmomente sowie auch Scherkräfte zwischen dem oberen und dem unteren Wirbelkörper übertragen.

Es ergeben sich sechs Elementarbewegungen der verbundenen Körpern: Drei Translationen $(\Delta \mathrm{x}, \Delta \mathrm{y}$ und $\Delta \mathrm{z})$ und drei Rotationen $\alpha_{\mathrm{X}}, \alpha_{\mathrm{Y}}, \alpha_{\mathrm{Z}}$. Die verbundenen Körper haben folgend in ihrer Bewegung sechs Freiheitsgrade (NÄGERL ET AL.1990).

\subsubsection{Diarthrosen}

Diarthrosen sind „echte" Gelenke und schränken die Bewegungsfreiheit und damit die Zahl der Freiheitsgrade ein. Anders als Synarthrosen übertragen sie nur Normalkräfte (FICK 1910a, b). Die biologischen Gelenkflächen sind inkongruent. Dies ist keine „Ungenauigkeit der Natur“ (FICK 1910a), sondern hat funktionelle Bedeutung. Einerseits ist die inkongruente Krümmung der artikulierenden Gelenkflächen die notwendige Voraussetzung für Schmierung und Ernährung des Knorpels durch Synovialflüssigkeit (MACCONAILL 1953, WALMSLEY 1928). Anderseits erlaubt diese Bauweise das Ausweichen von Stößen und führt dazu, dass die biologischen Gelenke unter Kraftschluss mechanisch selbststabilisierend wirken.

Als Beispiel besitzt ein Kugelgelenk zwei kongruente Artikulationsflächen, deren Krümmungsmittelpunkte zusammenfallen. Das Gelenk rotiert um drei Raum- 
achsen und hat drei Freiheitsgrade. Ein Scharniergelenk bewegt sich nur um eine Achse, die senkrecht zu Bewegungsebene steht (ein Freiheitsgrad). Eine Translation entlang der Scharnierachse wird blockiert.

Biologische Gelenke sind inkongruent. Die beiden Artikulationsflächen sind nicht formschlüssig. Um Führungsfunktion zu übernehmen, müssen die Gelenkflächen z.B. durch Muskelkräfte in Kontakt treten. Das Gelenk ist kraftschlüssig. Durch diesen Kraftschluss wird es in Funktion gesetzt (Abb. 1.4.4).

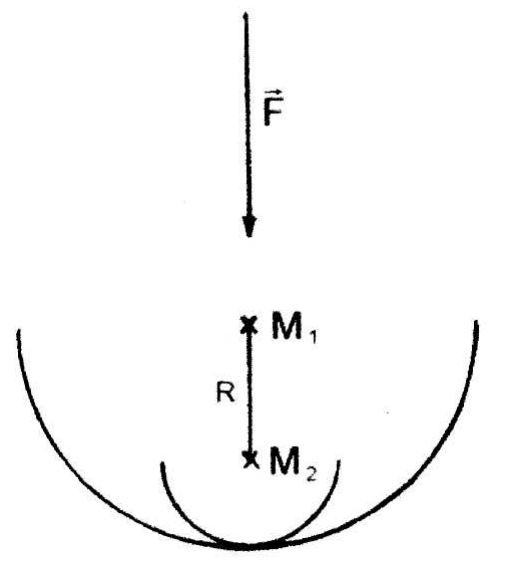
Abb. 1.4.4 Beispiel einer Diarthrose.
Kraftschluss durch kompressive Kraft $F$. Kraftwirkungslinie verläuft durch beide momentane Mittelpunkte $M_{1}$ und $M_{2}$

NäGERL (1990) wendete die Nomenklatur der technischen Gelenke zur Systematisierung biologischer Gelenke an (KUBEIN-MEESENBURG ET AL. 1993): Das Vorliegen einer Inkongruenz bei biologischen Gelenken hat die Erhöhung der Bewegungsfreiheit zur Folge. Zum Beispiel hat ein Kugelgelenk mit nur einem Kontaktpunkt nicht 3 sondern 5 Freiheitsgrade (das Metakarpophalangealgelenk; NÄGERL 1990), oder ein kraftschlüssiges Gelenk mit zwei Kontaktpunkten hat vier Freiheitsgrade (die Interphalangealgelenke oder das Tibiofemoralgelenk; NÄGERL ET AL.1993b, KUBEIN-MEESENBURG ET AL. 1991b). Sollten beide Wirbelbogengelenke eines Bewegungssegments der Wirbelsäule gleichzeitig führen, so reduzieren sich die fünf Freiheitsgrade jeweils eines Gelenks auf vier. Voraussetzung dafür ist der Kraftschluss. Es sind verschiedene Variationen der Kraftübertragung möglich. Ein Bewegungssegment könnte je nach Vorbelastung und Funktionszustand sechs, wenn kein Gelenk kompressiv belastet ist, fünf beim Kontaktpunkt in nur einem Gelenk oder vier Freiheitsgrade besitzen, im Fall wenn beide Gelenke kompressiv belastet sind (NÄGERL ET AL. 1995). 
Reibung und Gelenkschmierung

In Diarthrosen gleiten glatte Knorpeloberflächen aneinander vorbei. Zusammen mit der Gelenkschmierung wird die Haft- und Gleitreibung minimiert. Die inkongruenten Funktionsflächen bewirken bei der Bewegung einen „wandernden“ Gelenkspalt. Mit der elastischen Gelenkkapsel und den Menisken entsteht ein Pumpsystem, so dass die Synovia im bewegten Gelenk umgepumpt wird. Jede Stelle der knorpeligen Gelenkflächen kommt mit Synovialflüssigkeit in Kontakt und die Schmierung wird verbessert. Die Menisken oder meniskoide Falten verkleinern den Gelenkspalt und vergrößern den Pumpeffekt.

Ein weiterer denkbarer Mechanismus zur Minimierung der Reibung ist das Abrollen der Gelenkflächen. Das passiert, wenn die Führung des bewegten Körpers so gestaltet wird, dass die momentane Dreh-/ Schraubachse in der Kontaktfläche K zu liegen kommt. Dabei wird die Gleitreibung ausgeschaltet, weil die Gelenkflächen aneinander abrollen. Es resultieren nur oberflächensenkrechte Kräfte.

\section{Modelle der Gelenkführungen}

„Die kinematisch offene, dimere Gelenkkette“ mit einer konvexen und einer konkaven Gelenkfläche stellt das einfachste Modell für die Führung durch eine Diarthrose dar.

Eine „dimere Kugelgelenkkette“ ist die gelenkige Verbindung zweier artikulierender Gelenkflächen mit räumlich getrennten Krümmungsmittelpunkten (NäGERL ET AL.

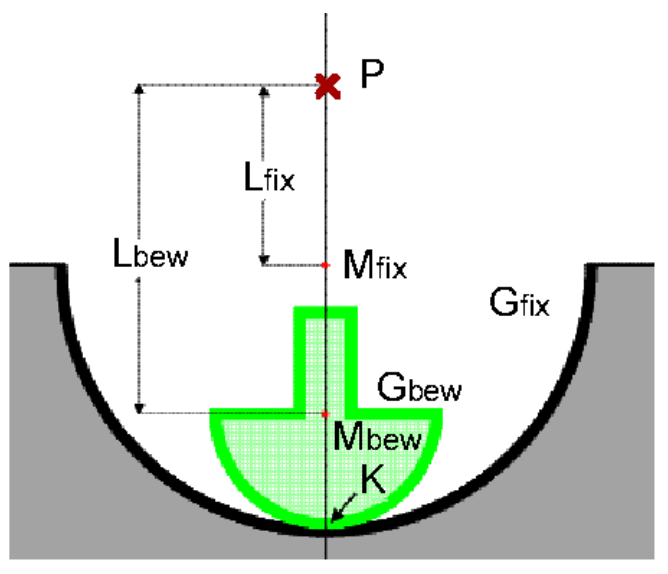

Abb. 1.4.5: Modell einer überschlagenden dimeren Gelenkkette $\left(\mathbf{G}_{\text {bew }}\right.$ ist grün markiert) 1993a, 1993b, Kubein-Meesenburg et al. 1990). Die Verbindung zweier Krümmungsmittelpunkte nennt man ein dimeres Kettenglied.

Zum besseren Verständnis sind die folgenden Beispiele einer überschlagenen Gelenkkette mit konkav-konvexen Gelenkflächen auf eine ebene Bewegung reduziert (Abb.1.4.5). Der absolute Drehwinkel $\alpha$ ist gleich dem Winkel zwischen 
den Verbindungslinien $\mathrm{M}_{\mathrm{fix}} \mathrm{M}_{\text {bew }}$ und $\mathrm{M}_{\mathrm{fix}} \mathrm{M}_{\text {bew. }}$.

Die konvexe bewegliche Gelenkfläche $\mathbf{G}_{\text {bew }}$ (Gelenkkopf) rotiert mit der Winkelgeschwindigkeit $\omega_{2}$ um den Krümmungsmittelpunkt $\mathbf{M}_{\text {bew. }}$. Die ortsfeste Gelenkfläche $\mathbf{G}_{\mathrm{fix}}$ (Gelenkpfanne) mit dem Krümmungsmittelpunkt $\mathbf{M}_{\mathrm{fix}}$ und der Winkelgeschwindigkeit $\omega_{1}$ kann sowohl um $M_{\text {bew }}$ als auch um $M_{\text {fix }}\left(\omega_{2}\right)$ rotieren. Die Verbindung $M_{\text {bew- }}$ $\mathrm{M}_{\mathrm{fix}}$ bezeichnet man als Kettenglied $\underline{\mathrm{m}}$. Der momentane Drehpol $\mathbf{P}$ einer Bewegung liegt immer auf der Geraden, die durch beide Krümmungsmittelpunkte verläuft. Das Winkelgeschwindigkeitsverhältnis $\omega_{1} / \omega_{2}$ gibt die momentane Drehachse $\mathbf{P}$ an. Sie steht senkrecht auf $\underline{m}$. Der Abstand von $P$ zu $M_{\text {bew }}$ sei $L_{\text {bew }}$ und zu $M_{\text {fix }}$ sei $L_{\text {fix }}$. Aus Quotienten ergibt sich:

$\mathrm{L}_{\text {bew }} / \mathrm{L}_{\text {fix }}=-\omega_{1} / \omega_{2}$ mit $\mathrm{L}_{\mathrm{fix}}-\mathrm{L}_{\text {bew }}=\underline{\mathrm{Im}} \mathrm{l}$.

Beide Gelenkflächen haben einen gemeinsamen Kontaktpunkt K.

Die Gelenkflächen können aufeinander gleiten oder abrollen. Beispiele von Elementarbewegungen einer dimeren Kette sind in Abb.1.4.6 A-D vorgestellt.

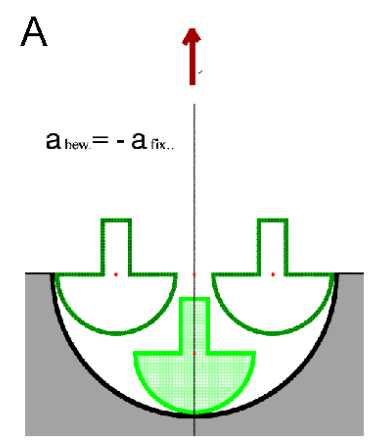

$\mathrm{B}$

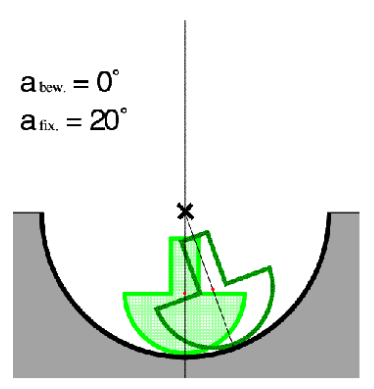

C

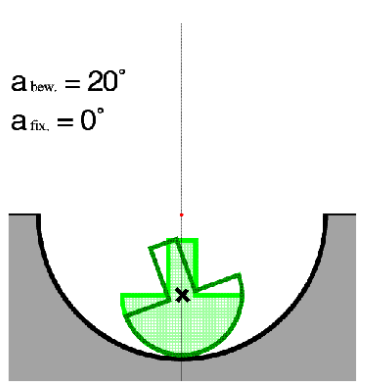

$\mathrm{D}$

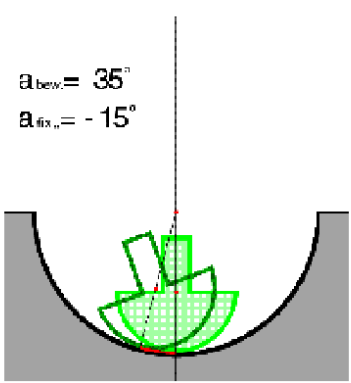

Abb. 1.4.6: Modell einer überschlagenen dimeren Gelenkkette vor und nach einer differentiell kleinen Elementarbewegung. A) Translation; B) und C): Gleiten; Rollen (D). Der bewegte Gelenkteil ist grün hervorgehoben.(MANSOUR 2001, S. 37)

Bei einer reinen Translation von $\mathrm{G}_{\text {bew }}(\mathbf{A})$ liegt die Drehachse im Unendlichen (mit rotem Pfeil hervorgehoben). Man kann die Art der Bewegung nach dem Verhältnis der Kontaktpunktwanderung auf beiden Gelenkflächen beschreiben. Bei einer reinen Gleitbewegung von $\mathrm{G}_{\text {bew }}$ um $\mathrm{M}_{\mathrm{fix}}$ in $\mathrm{G}_{\mathrm{fix}}(\mathrm{B})$ ist der Kontaktpunkt $\mathrm{K}$ auf der konkaven Gelenkfläche fix und wandert auf der anderen. Bei Gleitbewegung von $\mathrm{G}_{\text {bew }}$ um $\mathrm{M}_{\text {bew }}$ in $\mathrm{G}_{\text {fix }}$ liegt der fixe Kontakt $\mathrm{K}$ auf $\mathrm{G}_{\text {bew }}(\mathbf{C})$.

Der Weg des Kontaktpunkts $K$ auf $G_{\text {bew }}$ sei $d_{\text {bew }}$, und auf $G_{\text {fix }}$ sei $d_{\text {fix }}$.

Es gilt $d_{b e w} / d_{f i x}=0$ für eine reine Gleitbewegung. 
Beim Rollen findet eine gleichzeitige Rotation um $M_{\text {bew }}$ und $M_{\text {fix }}$ statt (D). Die resultierende Drehachse befindet sich in Kontakt K.

Für den Weg $d$ gilt $d_{\text {bew }} / d_{\mathrm{fix}}=1$.

In Abbildungen 1.4.7 A-D geht es um eine kombinierten Roll-/ Gleitbewegung. Der Kontaktpunkt $\mathrm{K}$ bewegt sich zwar auf beiden Gelenkflächen, aber der Weg $\mathrm{d}_{\text {bew }}$ muss mi dem Weg $\mathrm{d}_{\mathrm{fix}}$ nicht zwangsläufig übereinstimmen.

Hier gilt $d_{\text {bew }} / d_{\text {fix }}=0 \rightarrow 1$.

Bei dieser Bewegungskombination erhält man wandernde momentane Drehachsen. Folglich kann man mehrere Elementarbewegungen unterscheiden:

1. Gleichsinnige Rotation um Mfix und Mbew:

- die Rotation um Mbew überwiegt; P liegt zwischen K und Mbew (A)

- die Rotation um Mfix überwiegt; P liegt unterhalb von K (B)

2. Gegensinnige Rotation um Gfix und Gbew:

- die Rotation um $\mathrm{G}_{\mathrm{fix}}$ überwiegt; P liegt oberhalb von $\mathrm{M}_{\mathrm{fix}}(\mathbf{C})$

- die Rotation um $G_{\text {bew }}$ überwiegt.
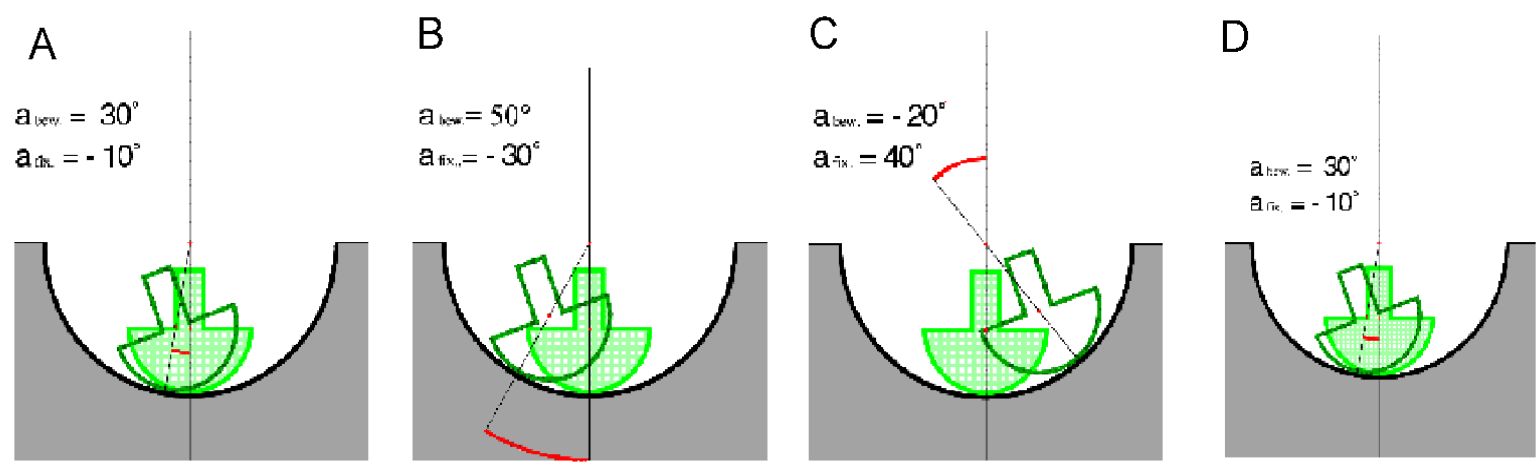

Abb. 1.4.7: Modell einer überschlagenen dimeren Gelenkkette vor und nach einer differentiell kleinen Elementarbewegung. A) Translation, B) und C) Gleiten D) Rollen. Der bewegte Gelenkteil ist grün hervorgehoben. Der Ort der momentanen Schraubachsen ist rot dargestellt.(MANSOUR 2001, S. 38) 


\subsubsection{Die Viergelenkkette als einfaches Modell bei beidseitiger}

Führung in komplexen Gelenken

Zwei Gelenke können durch zwei dimere Gelenkketten miteinander verbunden sein. Diese Verbindung bezeichnet man als Viergelenkkette.

In Allgemeinen beschreibt man die Gelenkflächen in der Brustwirbelsäule als zwei ineinander gestülpte Zylinder. Aufgrund einer physiologischen Inkongruenz fallen die Krümmungsmittelpunkte der Gelenkflächen nicht zusammen, somit werden die Artt. zygapophysiales superior und inferior zu zwei dimeren Ketten anatomisch fest verbunden. Man erhält das Viergelenk (Abb. 1.4.8).

In der Horizontalbewegung des oberen in Relation zum unteren Wirbels eines Junghannsschen Segments ist die Verbindungslinie $L_{G}$ der Krümmungsmittelpunkte $\mathbf{M}_{1 \text { fix }}$ und $\mathbf{M}_{2 \text { fix }}$ der konkaven Gelenkflächen $\mathbf{G}_{1 \text { fix }}$ und $\mathbf{G}_{2 \text { fix }}$ des fixierten unteren Wirbelkörpers das feste Gestell. Die bewegliche Koppel des oberen Wirbels ist die Verbindungsstrecke $\mathbf{L}_{\boldsymbol{K}}$ der Krümmungsmittelpunkte $\mathbf{M}_{\mathbf{1 b e w}}$ und $\mathbf{M}_{2 \text { bew }}$ der konvexen Gelenkflächen $\mathbf{G}_{1 \text { bew }}$ und $\mathbf{G}_{2 \text { bew. }}$

Gestell $L_{G}$ und Koppel $L_{K}$ sind funktionelle Größen und starr mit dem jeweiligen Wirbelkörper verbunden.

Treten die Gelenkflächen in Kontakt, ist der konstante Abstand ihrer Krümmungsmittelpunkte zueinander durch die Pleuelstange $\mathbf{R}_{\mathbf{1}}$ und $\mathbf{R}_{\mathbf{2}}$ gegeben. In der Gleichgewichtsstellung schneiden sich die durch $R_{1}$ und $R_{2}$ gegebenen Geraden im Ort der momentanen Drehachse P. Die Pleuel schneiden senkrecht die Gelenkflächen im Kontaktpunkt K.

In Ruhestellung liegt der momentane Drehpol der Koppel $L_{k}$ dorsal des Gestells.

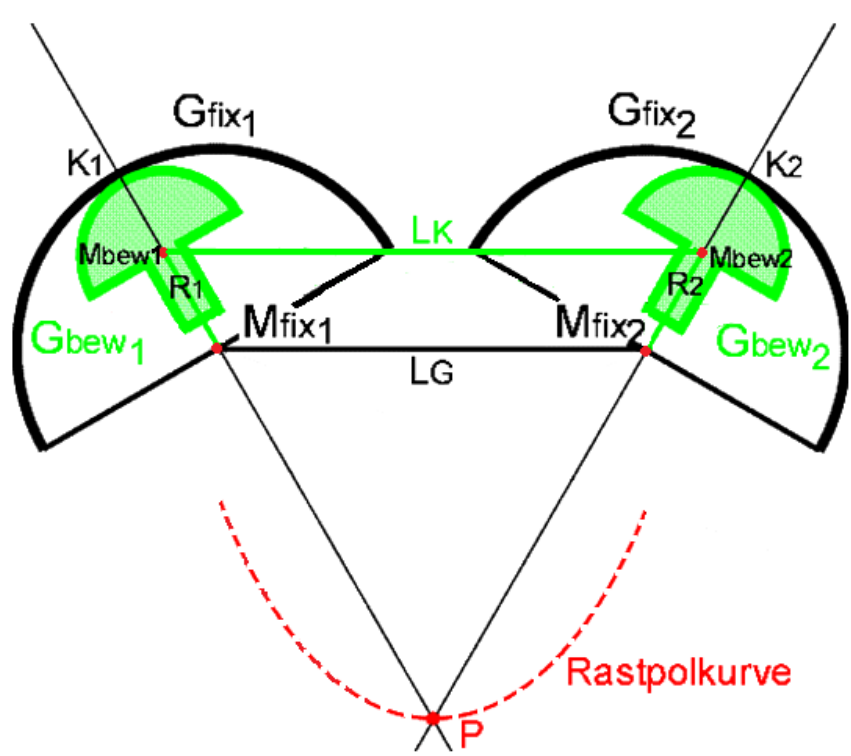

Abb. 1.4.8: Einfaches Modell einer Viergelenkkette. Der bewegte Gelenkteil ist grün hervorgehoben. Für die Krümmungsmittelpunkte (rot) und ihre Verbindungslinien sind die Positionen geometrisch konstruiert. Der Ort der momentanen Schraubachsen $\mathrm{P}$ und die Rastpolkurve bei differentieller Betrachtung sind rot dargestellt. 
Die momentane Drehachse $\mathrm{P}$ wandert im Laufe der Bewegung entlang eines nach dorsal offenen Bogens zwischen $\mathrm{M}_{01}$ und $\mathrm{M}_{02}$. Dieser Bogen ist die Rastpolkurve. Kommt es zur Rotation eines Pleuels um das Gestell, ist die Führung im System zwangläufig. Ein Viergelenk besitzt somit nur einen Freiheitsgrad.

Der Kraftschluss ist die Voraussetzung für die Funktion des Viergelenks. In Gleichgewichtsstellung setzt die Normalkraft $\underline{E}$ in $P$ an (Abb. 1.4.9), so verteilt sich Kraft $\underline{F}$ in zwei Lagerkräfte $\underline{F}_{1}$ und $F_{2}$ auf die beiden Kontaktpunkte. Es gilt für jede Kraft $F$ durch $P$ im Winkelbereich $\varphi$. Das Viergelenk bleibt mechanisch stabil. Es ändern sich nur die Komponenten $\underline{F}_{1}$ und $\underline{F}_{2}$.

Ein Parallelversatz der Kraft $\underline{F}$ richtet die Koppel $L_{K}$ entsprechend der neuen Kraftwirkungslinie auf, und $P$ wandert nach links. Dies gilt solange $F$ das Gestell $\mathrm{L}_{\mathrm{G}}$ schneidet. Schneidet die Kraftwirkungslinie jedoch nicht das Gestell, so wird eine Lagerkraft negativ. Der funktionelle Gelenkbereich wird überschritten. Das Gelenk wird auf Zug beansprucht und entkoppelt. Die Muskulatur hat die Aufgabe den Kraftvektor so zu positionieren, dass er immer die funktionelle Länge des Gestells schneidet.
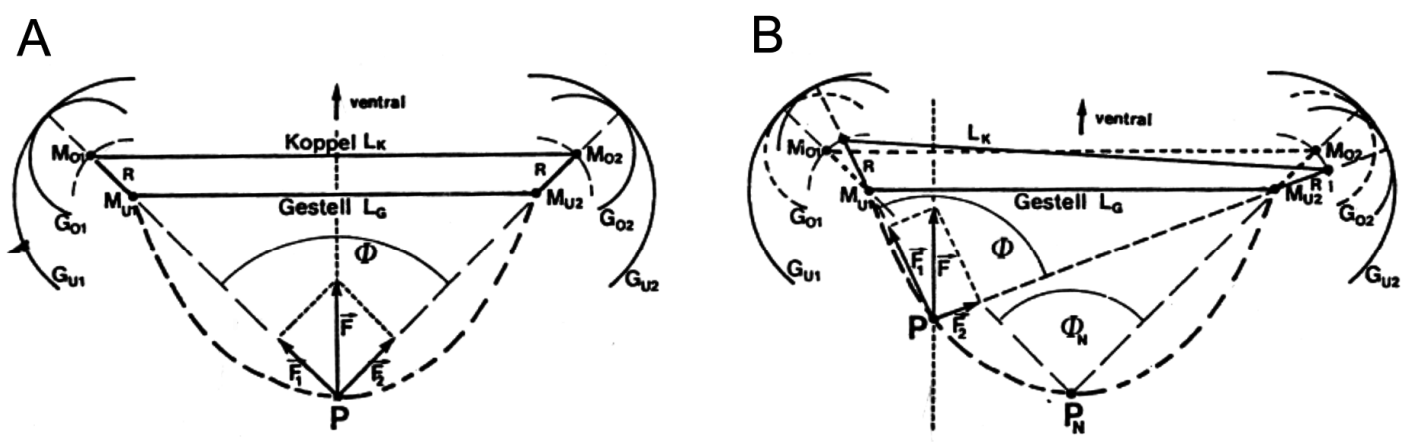

Abb. 1.4.9: Modell einer Viergelenkkette bei verschiedenen Kraftwirkungslinien.

In (A) teilt sich die Kraft $\underline{F}$ in zwei gleiche Komponenten. In (B) sind die Lagerkräfte $\underline{F}_{1}$ und $\underline{F}_{2}$ ungleich. Der bewegte obere Gelenkteil ist mit $01 / 2$ und der fixe untere mit U1/2 bezeichnet. Für die Krümmungsmittelpunkte und ihre Verbindungslinien sind die Positionen nach differentiellen Bewegungen geometrisch konstruiert (B). Der Ort der momentanen Schraubachsen $P$ und die Rastpolkurve (gestrichelt) sind bei differentieller Betrachtung dargestellt. 
Die anatomische Größe des Viergelenks gibt die Form der Rastpolkurve vor.

Die Abbildung 1.4.10 zeigt die denkbaren Viergelenkkonstellationen.

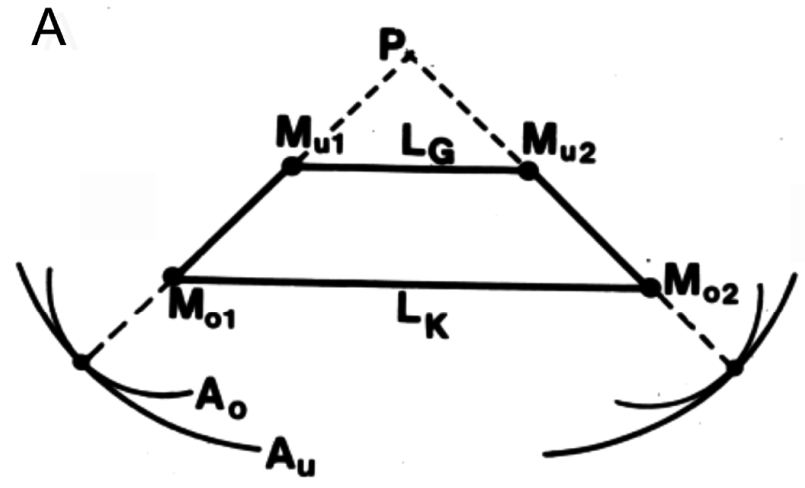

B
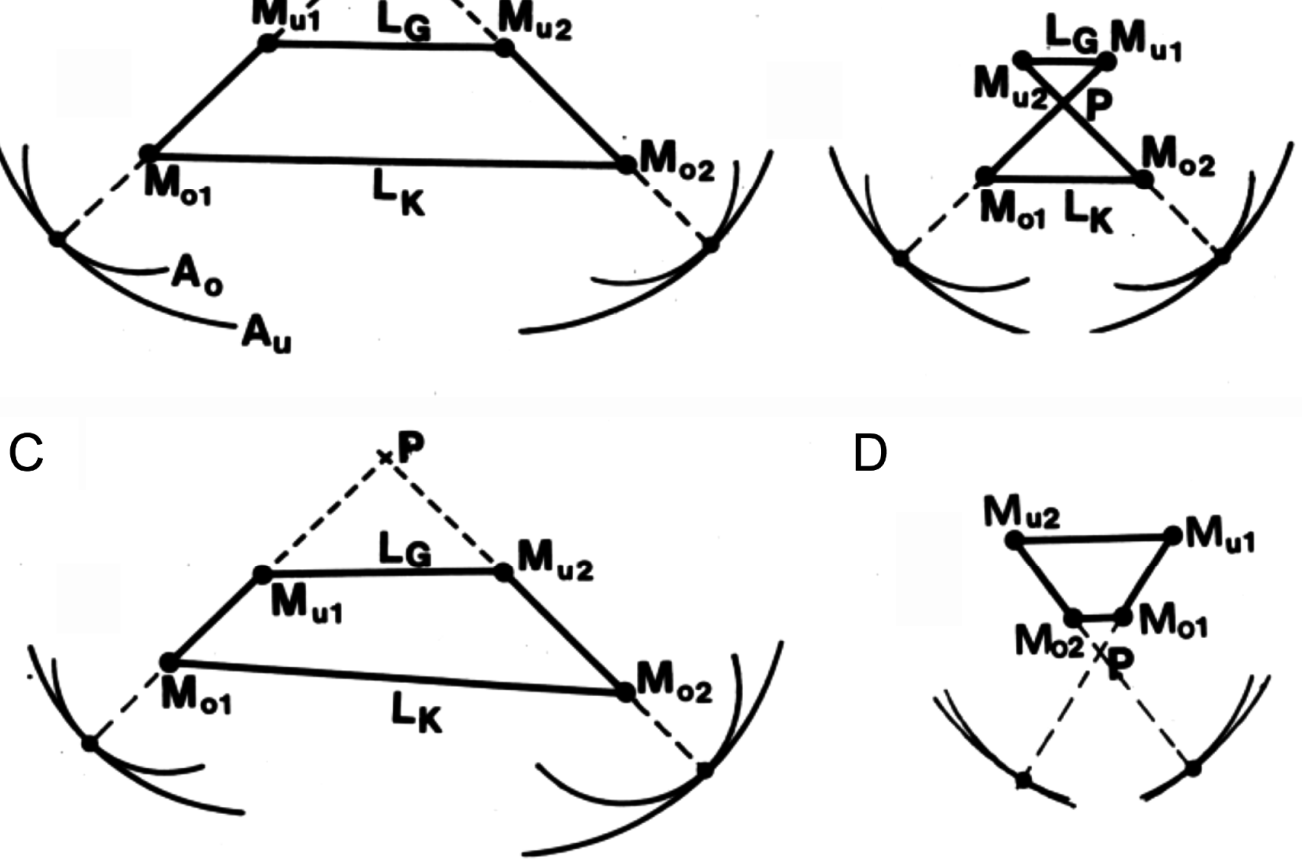

$\mathrm{D}$

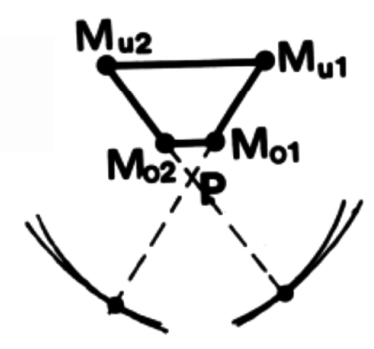

Abb. 1.4.10: Modelle möglicher Viergelenkketten.

Die Koppel $L_{k}$ verbindet die Krümmungsmittelpunkte des bewegten Anteils $\mathrm{M}_{01 / 2}$ und das Gestell $L_{G}$ die fixen Bestandteile $M_{U 1 / 2}$. Der Ort der momentanen Schraubachse ist $P$. Mögliche Beispiele für Viergelenke A) einfaches, symmetrisches; B) überkreuztes/ überschlagenes; C) asymmetrische und $\mathbf{D}$ ) invertiertes.

\subsubsection{Wechselwirkung von Synarthrose und Diarthrose}

Die kinematische Struktur des System Bandscheibe-Wirbelbogengelenks ist weitgehend durch die Wirbelbogengelenke bestimmt. Das Ausmaß der Bewegung ist hingegen von der Steifigkeit der Bandscheibe und der Lage der momentanen Drehachse abhängig (LEE 2003).

Die Vorstellung über die Interaktion zweier Gelenksysteme, einer viskoelastischen Bandscheibe und der beiden Wirbelbogengelenke ergibt sich aus den oben dargestellten Modellen. Vereinfacht wird hier zunächst nur von zwei beteiligten Diarthrosen und einer Synarthrose ausgegangen. 
Das Zusammenspiel des Systems folgt den in Kapitel 1.4.2 beschriebenen Steifigkeitsmodulen. Man setzt voraus, dass die Bandscheibe eine kreisrunde Scheibe mit dem Radius d, der Fläche $A=\pi \bullet d^{2}$ und der Dicke $h$ und homogener Elastizität ist (Abb.1.4.1, S. 29). Es gilt das Hook'sche Gesetz. Mehrere Bewegungstypen sind zu unterscheiden.

\section{Ebene Bewegung - Axialrotation}

Im Falle einer Axialrotation durch Anlegen eines Drehmoments $T_{z}$ um eine beliebige Drehachse P (parallel zur Symmetrieachse; keine Schraubsteigung) setzt sich die Elementarrotation $d \alpha_{z}$ aus einer Verschiebung ds (Translation) und einer Rotation $d \underline{\alpha}_{z}$ um eine durch das Widerstandszentrum $W_{z}$ verlaufende Achse $P_{w}$ mit $P$ II $P_{w}$ zusammen (Abb.1.4.11 A, S. 41). Der Abstand $P_{w}$ zu P sei $\underline{r}_{w}$.

Es gilt $d \underline{s}=d \underline{\alpha} z \times \underline{r} w$.

Steht $P$ senkrecht zur Bandscheibenebene, kommt es zur Verschiebung in $y$-Richtung dy durch die Scherkraft $d \underline{F}_{s}$. Schersteifigkeit der Bandscheibe ist Ds.

Es gilt: $\mathrm{dF}_{\mathrm{s}}=\mathrm{D}_{\mathrm{S}} \bullet \mathrm{d} \underline{\mathrm{s}}=\mathrm{D}_{\mathrm{S}} \bullet \mathrm{d} \underline{\alpha}_{\mathrm{Z}} \times \underline{\mathrm{ax}}$ oder $\mathrm{dF}_{\mathrm{s}}=\mathrm{D}_{\mathrm{S}} \bullet \mathrm{d} \alpha_{Z} \bullet \mathrm{ax}$.

Setz man voraus, dass sich das Gesamtsystem in der Achse $R$ abstützt und dort die Lagerkraft -dEs entsteht, wird die Abscherung durch ein äußeres Drehmoment erzeugt.

Es gilt $\mathrm{dT}_{\mathrm{s}}=\mathrm{ax}_{\mathrm{x}} \bullet \mathrm{dF}_{\mathrm{s}}=\mathrm{ax}_{\mathrm{x}} \bullet \mathrm{D}_{\mathrm{S}} \bullet \mathrm{d} \alpha_{\mathrm{z}} \bullet \mathrm{ax}_{\mathrm{x}}=\mathrm{D}_{\mathrm{S}} \bullet \mathrm{d} \alpha_{\mathrm{z}} \bullet \mathrm{ax}^{2}$.

Sind beide Gelenke kraftschlüssig, schneiden sich die Lagerkräfte in der Drehachse $P$, mit Resultante $-\mathrm{dF}_{\mathrm{s}}$. In dem Fall entspricht die Abscherung der Drehsteifigkeit $d T_{s} / d \alpha_{z}=D_{s} \bullet a_{x}^{2}$. Für diese Kombinationsbewegung ist ein zusätzliches Drehmoment $d T_{Z}$ für die Rotation um $R_{W}$ erforderlich. Bei dieser Bewegung wird das System auf Torsion beansprucht. Die Torsionsteifigkeit $D_{T}$ ist $d T_{Z} / d \alpha_{Z}=D_{T}$. Die beiden Steifigkeiten sind parallel geschaltet. $D_{G}$ ist die Gesamtsteifigkeit des Systems und setzt sich aus der Torsionssteifigkeit $\mathrm{D}_{\mathrm{T}}$ (mit dem Torsionsmodul) und der Schersteifigkeit $\mathrm{D}_{\mathrm{S}}$ (mit dem Schermodul) zusammen.

Es gilt $d T_{Z} / d \alpha_{Z}=D_{G}=D_{T}+a_{x}{ }^{2} \bullet D_{S}$

Bei einer Axialrotation erfährt die Bandscheibe keine Zug- oder Druckbelastung, somit spielt das Elastizitätsmodul E der longitudinalen Dehnung hier keine Rolle. Bei Verkippung $\alpha_{Y}$ um eine zur y-Achse parallele Drehachse P mit dem Abstand 
$r_{\mathrm{w}}$, kommt es zu einer Dehnung/ Kompression der Bandscheibe in Abhängigkeit des Elastizitätsmoduls $\mathrm{E}$ (Abb. 1.4.11 B):

Es gilt $d T_{Y} / d \alpha_{Y}=D=D_{T}+a_{X}{ }^{2} \bullet D_{E}$.

A

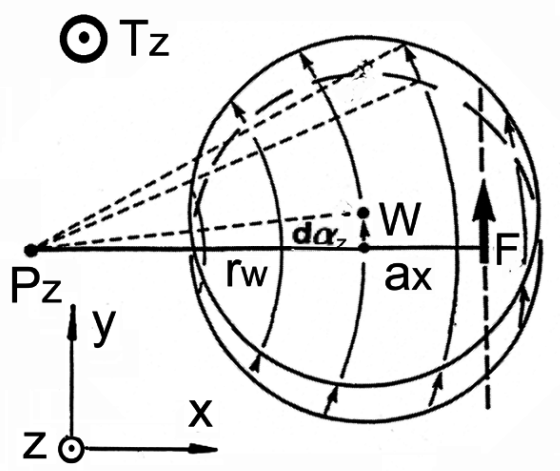

B

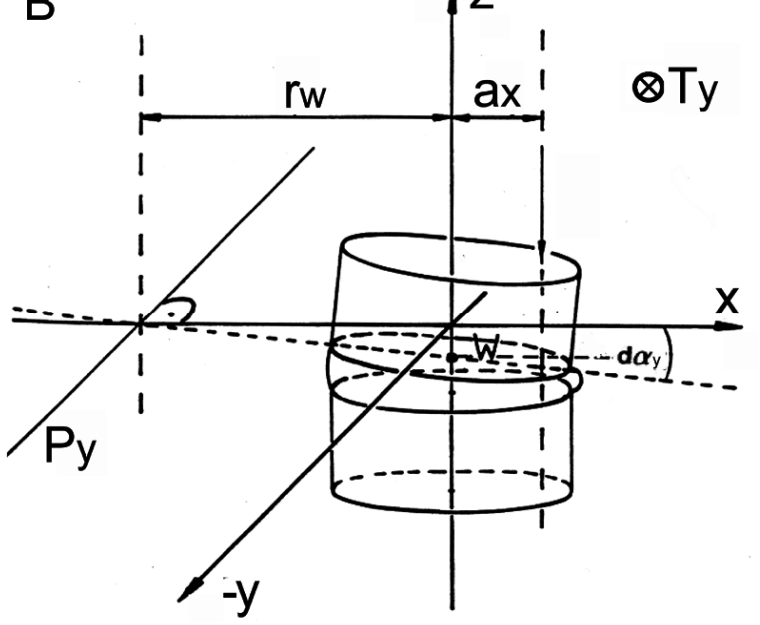

Abb. 1.4.11: Wirkung eines axialen Drehmoments $T_{Z}(\mathbf{A})$ und $T_{y}(B)$ auf das Bewegungssegment. Es resultiert die Rotation daz bzw. day um eine Drehachse $P_{z / y}$. Rw ist Abstand Drehachse $P$ vom Widerstandszentrum W. Es wirkt eine Kraft $\underline{F}$ auf die Bandscheibe. Daraus resultiert eine Gegenkraft - $\underline{F}$, die der Abscherung (A) bzw. Kompression (B) entgegenwirkt.
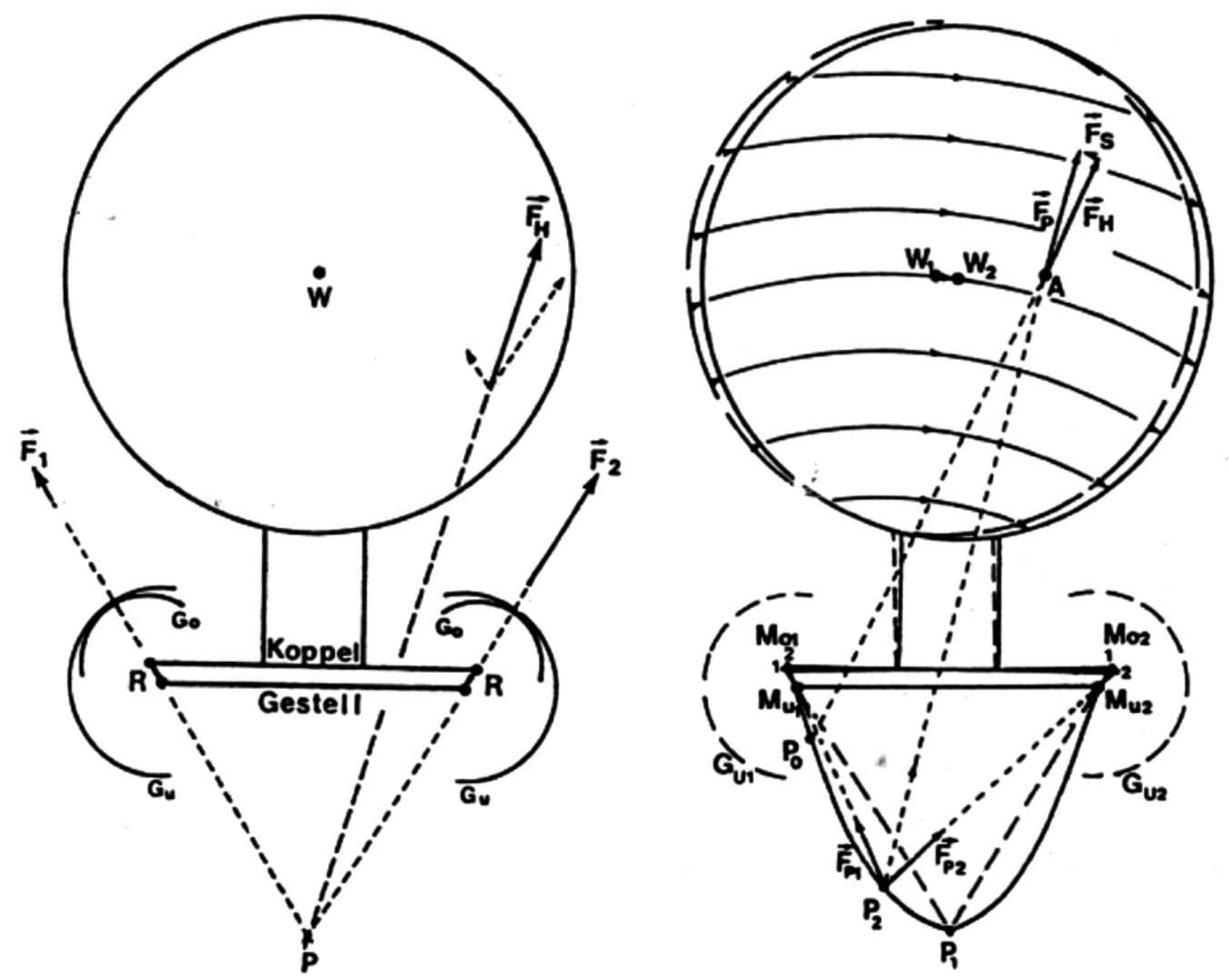

Abb. 1.4.12 : Ebene Bewegung eines Bewegungssegments. Es resultiert eine Rotation doz bzw. day um eine Drehachse außerhalb der Bandscheibe. Eine Kraft $\underline{F}_{S}$ erzeugt eine Gegenkraft $-\underline{F}$, die der Abscherung entgegenwirkt. Es resultieren die Lagerkräfte $F_{P 1}$ und $F_{P 2}$, die sich in $\mathrm{P}$ schneiden. 
Verschraubung bei senkrechter Schraubachse

Kommt es bei Axialrotation $\alpha_{z}$ zusätzlich noch zum Versatz um ds entlang der Rotationsachse P im Sinne einer Verschraubung $\tau(\alpha)$, wird die Bandscheibe gestaucht oder gedehnt. Zur Gesamtsteifigkeit $\mathrm{D}_{G}$ addiert sich das Elastizitätsmodul.

Es gilt $\tau=\tau(\alpha)=\tau_{0}$ und $\tau=\tau(\alpha)=A \cdot \alpha$.

Für den Versatz gilt $d s=\tau(\alpha) \cdot d \alpha$.

Für den Energiezuwachs, als elastische Energie gespeichert gilt $d W=F(s) \cdot d s$. Außerdem ist der Kraftansatz vom Elastizitätsmodul $D_{E}$ und der Höhe des Versatzes ds abhängig $d F(s)=D_{E} \cdot d s$.

$\mathrm{F}(\mathrm{s})=\mathrm{D}_{\mathrm{E}} \cdot \int_{0}^{\mathrm{s}} \mathrm{ds}=\mathrm{D}_{\mathrm{E}} \cdot \int_{0}^{\infty} \tau(\alpha) \cdot \mathrm{d} \alpha \Rightarrow$

$\mathrm{dW}=\mathrm{F}(\mathrm{s}) \mathrm{ds}=\mathrm{D}_{\mathrm{E}} \cdot\left(\int_{0}^{\mathrm{s}} \mathrm{ds}\right) \cdot \mathrm{ds}=\mathrm{D}_{\mathrm{E}} \cdot\left(\int_{0}^{\alpha} \tau(\alpha) \cdot \mathrm{d} \alpha\right) \cdot \tau(\alpha) \cdot \mathrm{d} \alpha$.

Der Energiezuwachs $d W$ wird durch die Rotation erzeugt. Das dafür benötigte

Drehmoment ist $d W / d \alpha=T(\alpha)$ :

$\frac{\mathrm{dW}}{\mathrm{d} \alpha}=\mathrm{T}(\alpha)=\mathrm{D}_{\mathrm{E}} \cdot\left(\int_{0}^{\alpha} \tau(\alpha) \cdot \mathrm{d} \alpha\right) \cdot \tau(\alpha)$
$\frac{\mathrm{dT}}{\mathrm{d} \alpha}=\mathrm{D}_{\mathrm{rsch}}=\mathrm{D}_{\mathrm{E} \cdot} \cdot\left[\tau^{2}(\alpha)+\frac{\mathrm{d} \tau(\alpha)}{\mathrm{d}(\alpha)} \cdot \int_{0}^{\alpha} \tau(\alpha) \cdot \mathrm{d} \alpha\right]$.

Für $\tau(\alpha)=\tau_{0}$ folgt: $\frac{\mathrm{dT}}{\mathrm{d} \alpha}=\mathrm{D}_{\mathrm{rsch}}=\mathrm{D}_{\mathrm{E}} \cdot \tau_{\mathrm{o}}^{2}$

Für $\tau(\alpha)=R \cdot \alpha$ gilt: $\quad \frac{\mathrm{dT}}{\mathrm{d} \alpha}=\mathrm{D}_{\mathrm{rsch}}=\mathrm{D}_{\mathrm{E}} \cdot\left[\mathrm{A}^{2} \cdot \alpha^{2}+\mathrm{A}^{2} \cdot \frac{\alpha^{2}}{2}\right]=\frac{3}{2} \cdot \mathrm{D}_{\mathrm{E}} \cdot \mathrm{A}^{2} \cdot \alpha^{2}$.

Bleibt die Schraubsteigung konstant, addiert sich auch ein konstanter Anteil der Steifigkeit hinzu. Steigt die Schraubsteigung linear an, kommt ein quadratischer Term hinzu.

Schraubachse ist nicht senkrecht zu Bandscheibenebenen

In diesem Fall sind die oben beschriebenen Elementarbewegungen nicht mehr möglich. Es kommt zur Kombinationsbewegungen, die nun sowohl von reiner Torsionssteifigkeit $D_{T}$ mit dem Schermodul $G$ als auch von einer Biegesteifigkeit $D_{E}$ bestimmt werden. Die Gesamtsteifigkeit des Systems erhöht sich. 
Lagerkräfte sind windschief

Bei gleichsinnig gekrümmten Gelenkflächen schneiden sich die Kraftwirkungslinien der beiden Gelenkkräfte in der Drehachse P. Es resultiert die Auflagerkraft

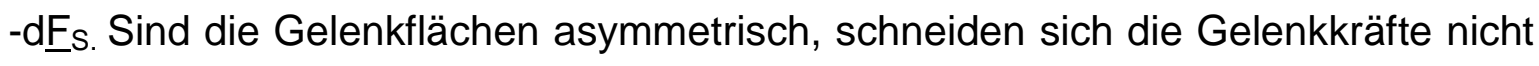
mehr. Sie sind windschief. Dadurch wird ein rücktreibendes Drehmoment $-T_{R}(\alpha)$ erzeugt, der mit dem Drehwinkel $\alpha$ ansteigt. Die Steifigkeit des Bewegungssegments erhöht sich. 


\section{Material und Methoden}

Es sind zahlreiche Untersuchungen der Bewegung der Wirbelsäule bekannt, jedoch befassen sich nur wenige implizit mit der Brustwirbelsäule. Die meisten Untersuchungen sind auf Hals - oder Lendenwirbelsäulenabschnitte begrenzt.

Die in der Göttingener Arbeitsgruppe für Biomechanik eingesetzte Messmethode ist auch auf Brustwirbelsäulenabschnitte übertragbar (LEE 2003). Die Methode wird in folgenden Kapiteln ausführlich beschrieben.

\subsection{Messprinzip}

Ziel dieser Arbeit war es, eine exakte Analyse von der räumlichen Bewegung der beiden Wirbel eines Brustwirbelsäulensegments (JUNGHANNSSCHE SEGMENT) in der Höhe T2-T3 zu beschreiben. Es wurde mit Hilfe eines zeitlich veränderlichen, räumlichen Kraftsystems hochaufgelöst, mit einem sechsdimensionalen Messsystems, die Bewegungsstruktur aufgenommen, um sie mathematisch zu analysieren und mit den anatomischen Gegebenheiten zu vergleichen. Durch die Verbesserung des dazu erforderlichen Mess- und Analysesystem wurde eine höhere räumliche und zeitliche Auflösung des Bewegungsvorgangs und die Applikation eines Drehmoments $T_{X}$ und $T_{Y}$ ermöglicht. Damit wurde die Analysetechnik verfeinert und verschiedene Parametern wurden definiert, um die entstandene Bewegungsmuster interpretierend und zu vergleichen.

\section{Experimentelle Realisation:}

- Messung der Lageänderung des oberen Wirbelkörpers am Bewegungssegment T2/ T3, T3-Wirbel ist fixiert

- Simulation einer Kraftschraube durch Applikation eines zyklischen, zeitlich einer Dreiecksfunktion folgenden Drehmoments auf den beweglichen T2Wirbel in X-, Y- und Z-Richtung. Die Vorlastgrößen und Positionen wurden variabel eingestellt 
- Erfassung der Momentanbewegung durch ein System mit sechs Induktionstastern mit einer Genauigkeit von 0,01- 0,24 $\mu \mathrm{m}$

- Darstellung der räumlichen momentanen Bewegung über die Berechnung momentaner Schraubachsen

- Zuordnung der sechs Schraubachsparameter und der Rastregelflächen zu den morphologischen Gegebenheiten

- Applikation einer Kraftschraube jeweils am intakten Segment, nach sukzessiver Resektion der Artt. zygapophysiales.

\subsection{Material und Morphologie}

\subsubsection{Brustwirbelpräparate}

Das Autopsiematerial für diese Studie wurde vom Anatomischen Institut der Universität Greifswald zur Verfügung gestellt. Die Wirbelsäulenpräparate wurden mit einem bänderkonservierenden Formalin-Alkohol-Thymol-Glycin-Gemisch fixiert. Dieses schonende Konservierungsverfahren erhält weitgehend die physiologischen Eigenschaften der Bänder. Eine Kontraktion oder Versteifung durch Formalin wird durch diese Lösung vermieden (FANGHÄNEL UND SCHULZ 1962). Die zwei Brustwirbelsäulenpräparate wurden „en bloc“ entnommen und die jeweiligen Th 2/3 Segmente unter Erhaltung der Bänder sorgfältig präpariert. Das Wirbelsäulenpräparat mit der Bezeichnung $\mathbf{A}$ stammt von einer männlichen Person (Alter 54 Jahre), das zweite, als B bezeichnet, von einer weiblichen Person (Alter 87 Jahre). Mithilfe der Röntgenaufnahmen und auch mittels Feinschicht-Computertomographie-Aufnahmen (Spiral-CT, Toshiba Express) wurden pathologische Veränderungen an den Zwischenwirbelscheiben und an den Artt. zygapophysiales ausgeschlossen (Abb. 2.2.1 A/B, S. 46). 

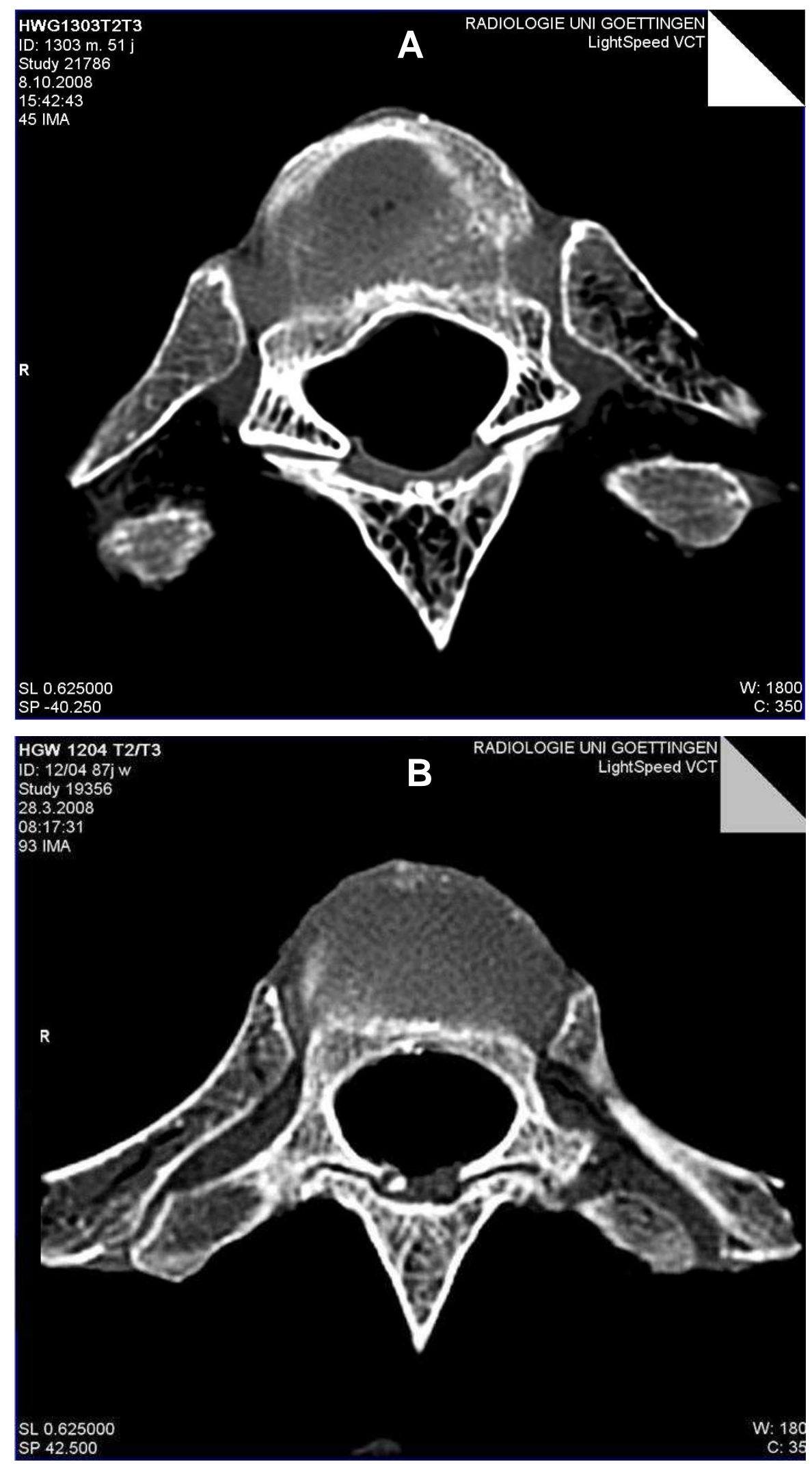

Abb. 2.2.1 A, B: CT- Aufnahmen von T2/ T3 A (oben) und T2/ T3 B (unten). Die Bilder wurden mit 120 kV, 120 mA bei einer Schichtdicke von 2 mm und einem Schichtabstand von 1,5 mm aufgenommen. 


\subsubsection{Einbettung}

Um eine direkte Kraftübertragung zu gewährleisten sowie auch eine Verbindung des Bewegungssegmentes mit der Messapparatur zu sichern, wurden die Wirbelkörper in einer Wanne aus Stahl mit kaltpolymerisierendem Kunststoff Combipress ${ }^{\circledR}$ eingegossen. Die Gelenke und die Bandscheibe blieben dabei frei. Der dorsale Teil der Zwischenwirbelscheibe wurde mittig in der Wanne positioniert, da hier das Widerstandszentrum vermutet wurde (NÄGERL 1990). Die Bandscheibenebene wurde parallel zum Wannenboden und die Medianebene parallel zum seitlichen Wannenrand ausgerichtet. Ein Führungsgestell gewährleistet die planparallele Position der Wannen zueinander beim Einbetten. Zur sicheren Fixation der Wirbelkörper in dem Einbettkunststoff wurden die Wirbelkörper mit Schrauben versehen. Die vier Flügelmuttern wurden ebenfalls eingegossen, dadurch konnte das Präparat positionsstabil an der Stahlwanne fixiert werden. Während der Messung wurde das eingebettete Segment immer wieder mit Fixationslösung besprüht, um eine Austrocknung zu vermeiden, und in der Zeit zwischen den Messungen in Lösung getränkt und kühl gelagert. Polymerisationskunststoff Combipress $\AA_{\text {a }}$ wird von der Konservierungslösung nicht angegriffen und ist durchlässig für Röntgenstrahlen.

Abb. 2.2.2 A: T2/3 B a-p

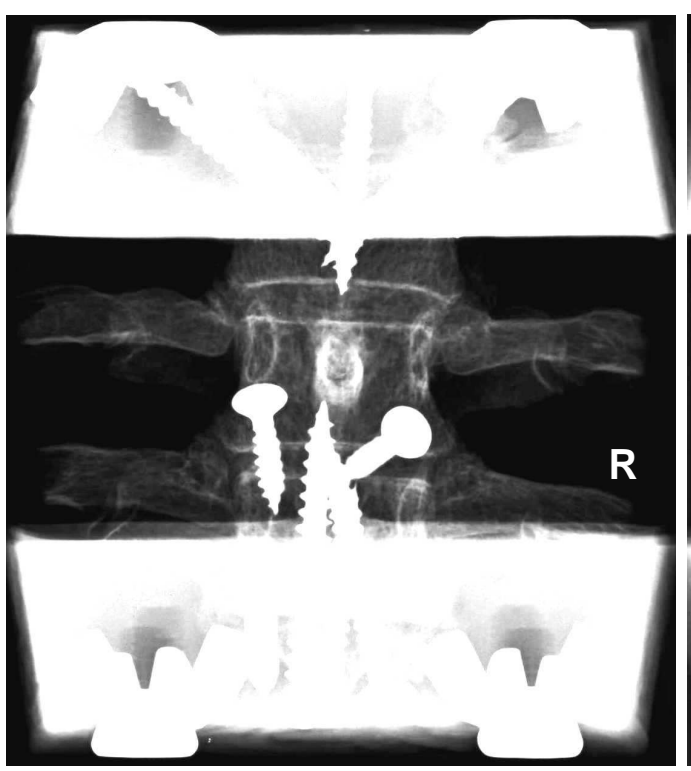

Abb. 2.2.2 B: T2/3 B (links) lateral.

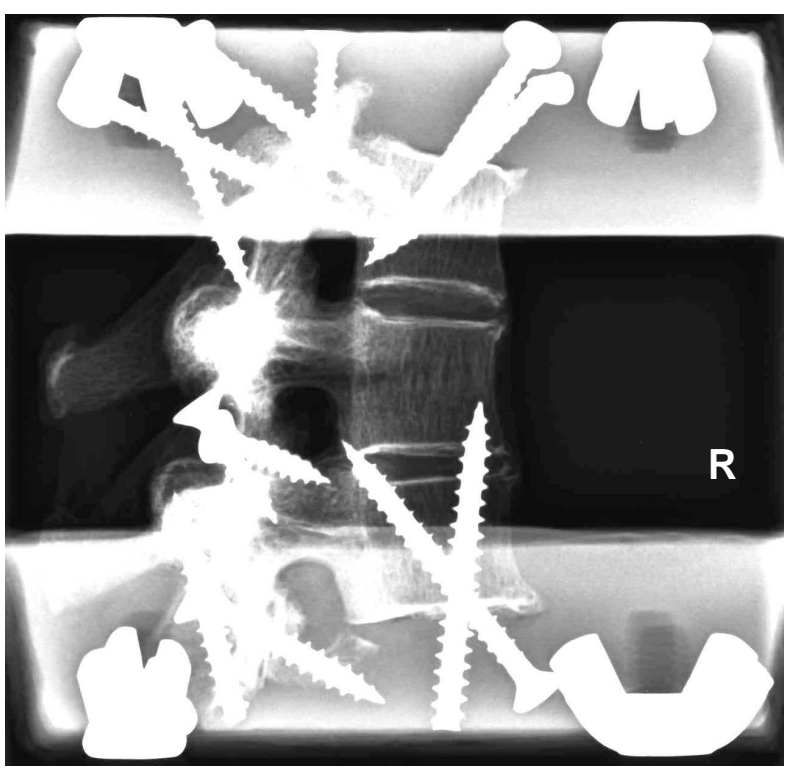


Abb. 2.2.2 C: T2/3 A a-p

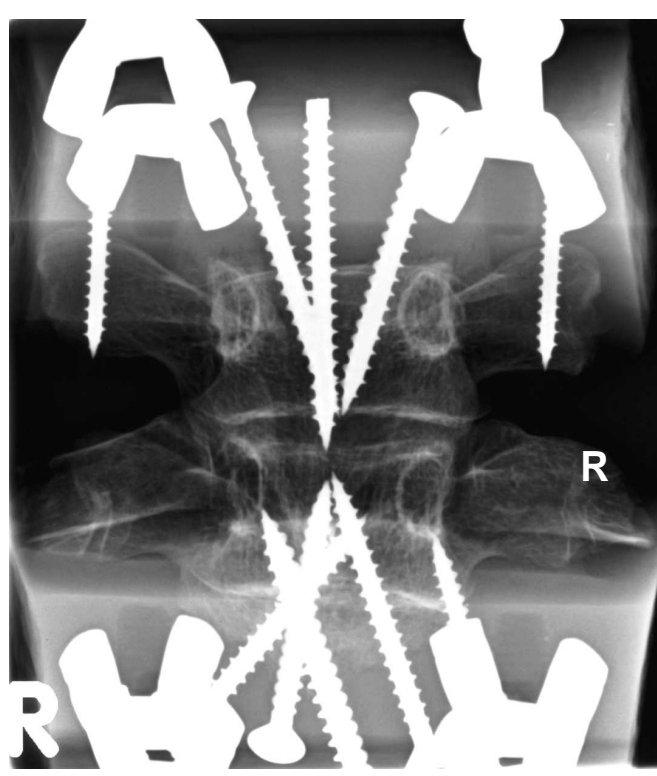

Abb. 2.2.2 D: T2/3 A (links) lateral.

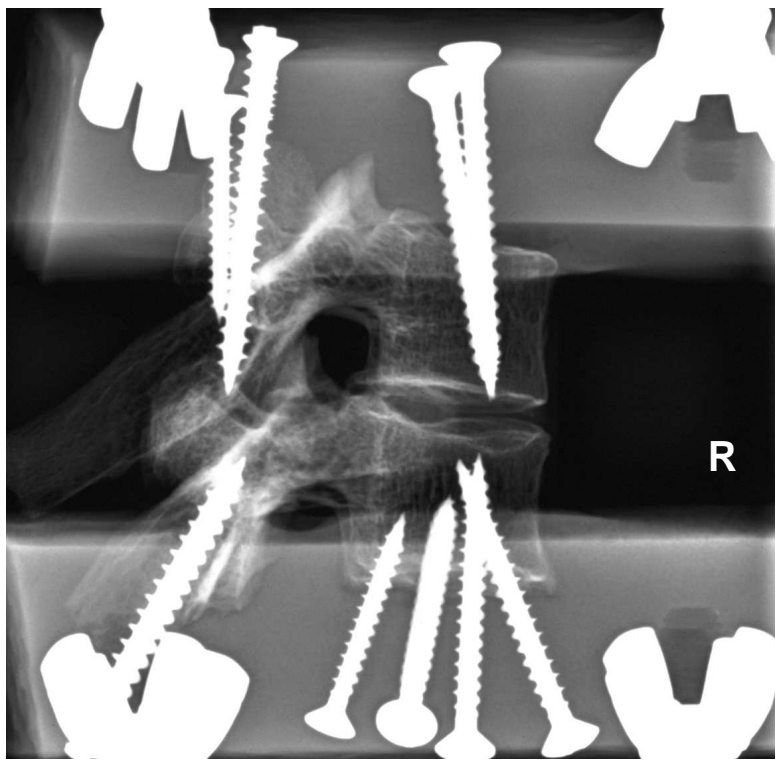

Abb. 2.2.2: Röntgenaufnahmen der beiden eingebetteten, intakten Brustwirbelsegmente;

Abb.2.2.2 A und B, jeweils eine pa und seitliche Aufnahme des eingebetteten Segments T2/3 B Abb.2.2.2 $C$ und $D$, jeweils eine pa und seitliche Aufnahme des eingebetteten Segments T2/3 A

\subsubsection{Zuordnung von morphologischen Strukturen zum Messsystem}

Hochauflösende Röntgenaufnahmen (40 kV, $8 \mathrm{mAs})$ in zwei Ebenen für beide Wirbelsäulensegmente (Abb. 2.2.2) verifizierten die Positionierung und Ausrichtung der Wirbel in ihren Wannen und bestätigten die Unversehrtheit knöcherner Strukturen.

Die CT-Aufnahmen wurden für eine räumliche Zuordnung der erhaltenen Ergebnisse zu realen anatomischen Gegebenheiten genutzt. Das Ausmessen der CTAufnahmen eines Präparates im dreidimensionalen Raum erfolgte durch die Bestimmung deren Höhe im Raum aus den zweidimensionalen Schichtaufnahmen und des Schichtabstandes dieser Aufnahmen. Anhand dieser Daten wurden nun die Ergebnisgrafiken der jeweiligen Strukturen hineinprojiziert, um die Bewegungsstruktur des Segmentes leichter nachzuvollziehen und zu veranschaulichen (WACHOWSKI 2005). Die Größe und Position von Bandscheibe und Wirbelgelenken im Messsystem wurde bestimmt. Die Dicke einer Schichtaufnahme beträgt 1,5 mm, so dass eine Darstellung des Gelenkes möglich wird (Abb. 2.2.1, S. 46). An- 
hand der angefertigten CT-Bilder des eingebetteten Segmentes ergab sich die folgende Zuordnung der anatomischen Strukturen in Bezug zum Nullpunkt der Messapparatur bzw. dem Widerstandszentrum.

\section{Segment TH2/3 A}

Gerechnet vom Mittelpunkt der Wanne liegt die

Bandscheibenmitte:

Vorderer Rand der Bandscheibe:

Hinterer Rand der Bandscheibe:

Wirbelbogenkanal:

Wirbelbogengelenke:

Proc. spinosus:

\section{Segment TH2/3 B}

Bandscheibenmitte:

Vorderer Rand der Bandscheibe:

Hinterer Rand der Bandscheibe:

Wirbelbogenkanal:

Wirbelbogengelenke:

Proc. spinosus:

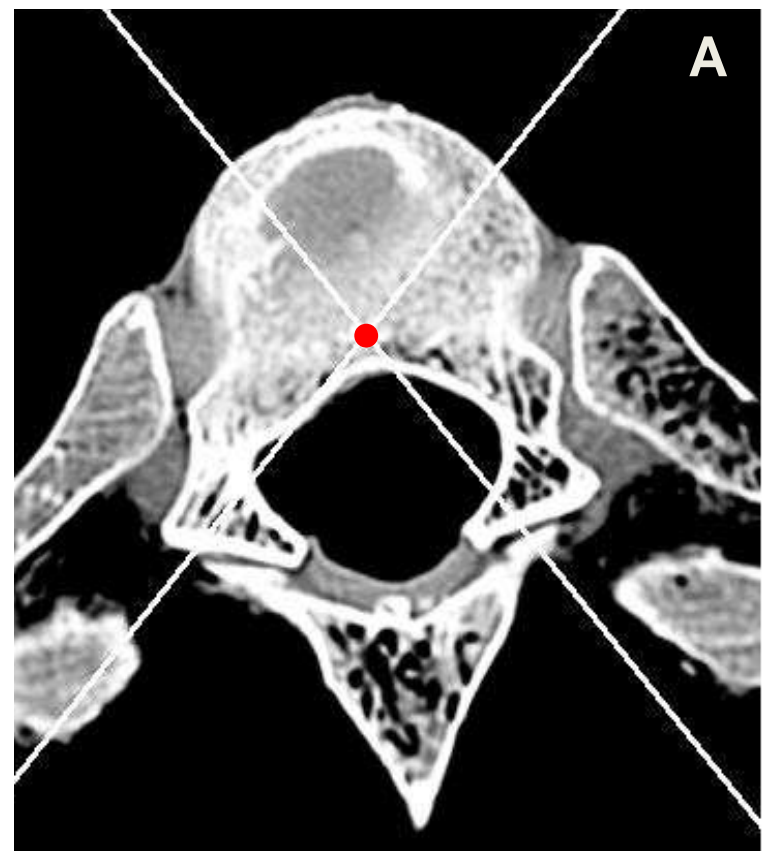

ventral $8 \mathrm{~mm}$

ventral $20 \mathrm{~mm}$

dorsal $4 \mathrm{~mm}$

dorsal $13 \mathrm{~mm}$

dorsal $22 \mathrm{~mm}$

dorsal $44 \mathrm{~mm}$

ventral $5 \mathrm{~mm}$

ventral $18 \mathrm{~mm}$

dorsal $5 \mathrm{~mm}$

dorsal $15 \mathrm{~mm}$

dorsal $22 \mathrm{~mm}$

dorsal $41 \mathrm{~mm}$

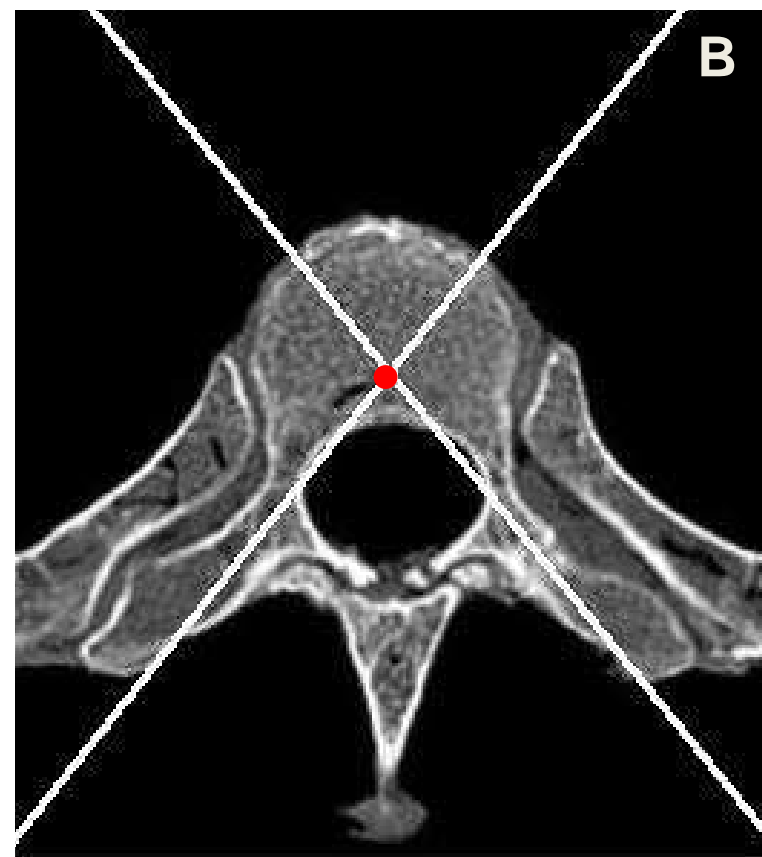

Abb. 2.2.3 A, B: CT- Aufnahmen des eingebetteten Segmentes Th2/3 A (A) und Th2/3 B (B) Die Wannenmitte ist mit einem roten Punkt markiert und entspricht dem Nullpunkt der Apparatur. Der Nullpunkt stimmt annähernd mit ermittelten Widerstandszentrums in beiden Fällen überein. 


\subsection{Messapparatur}

Die von SPIERING (1995) entwickelte Messapparatur besteht aus einem Stahlrahmen als Grundgerüst. Die Vierkantstangen sind schwingungsgedämpft gelagert (Abb. 2.3.1). Zur Vermeidung von Eigenschwingungen ist das Messsystem selbst auf einer massiven Metallgrundplatte von $50 \times 50 \mathrm{~cm}$ montiert.

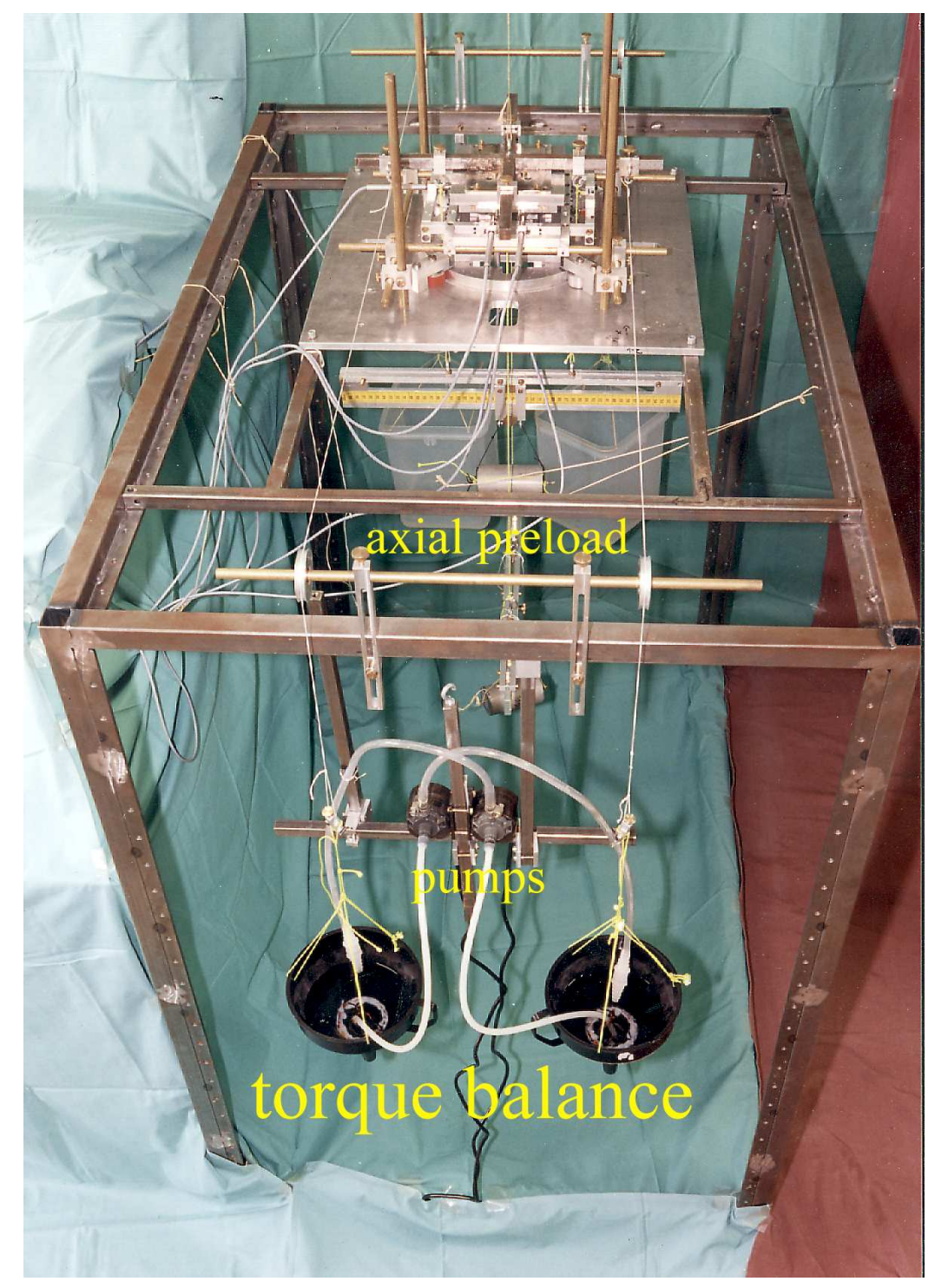

Abb. 2.3.1: Blick auf den Messstand von oben vorne: Oben im Bild das eingebettete Präparat, in der Mitte unten die axiale Vorlast (axial preload) und im Vordergrund das zur Drehmomentwaage (torque balance) gehörende Pumsystem (pumps).

Computer und Messverstärker sind nicht abgebildet. 
In der unteren Stahlwanne (Abb. 2.3.2) ist der Wirbel Th3 des eingebetteten Segments an der Grundplatte fixiert, während in der oberen Stahlwanne der Th2Wirbel mit dem Kraft- und Messsystem verbunden ist. Das Kraftaufbringungs- und Messsystem setzt sich aus einem Stahlkreuz, das die Drehmomente und die axiale Vorlast rückwirkungsfrei auf das Objekt überträgt, und einem an der oberen Wanne platzierten Gerüst mit 6 Lagersensoren zusammen. Durch diese sechs Induktionstaster wurden die resultierende relative Bewegungen des oberen Wirbels und somit der oberen Stahlwanne gegen Th3 aufgenommen. Das Eigengewicht der oberen Wanne und des Kraftübertragungskreuzes wird über ein rollengelagertes Gegengewicht kompensiert.

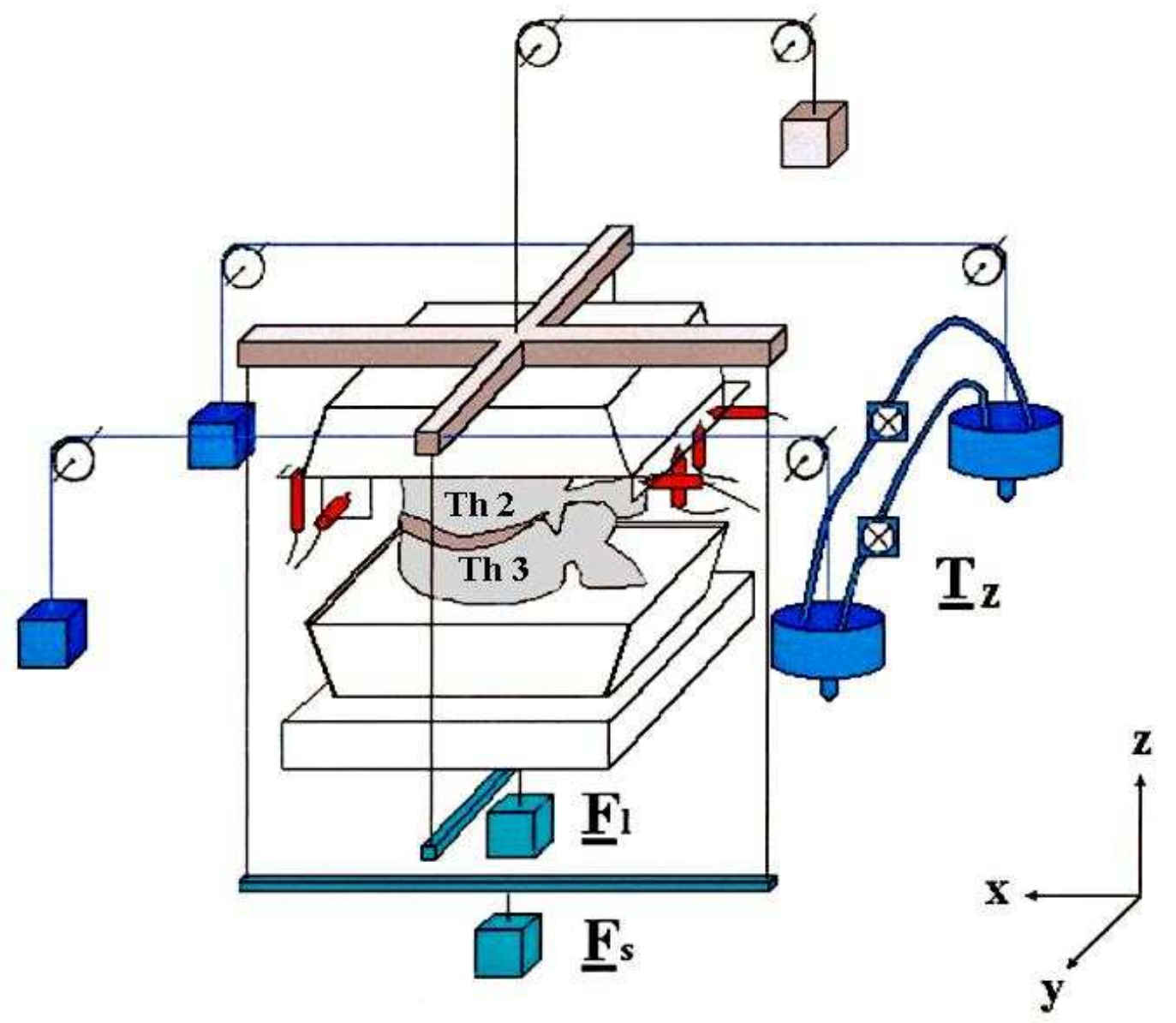

Abb. 2.3.2: Schema des Messstands (Ansicht von links). 


\subsubsection{Aufbau des Messsystems}

In einem in Abbildung 2.3.3 abgebildeten Metallrahmen sind sechs Messtaster fixiert. Die Anordnung der Messtaster entspricht dem „3-2-1 System“ (Abb. 2.3.4, S. 53). Die Aufzeichnung von der relativen Bewegung des oberen Wirbels erfolgt über die den Messflächen entsprechenden und deren anliegenden Tasterspitzen. Die Messflächen sind glasbeschichtet. Der Tasterhalterahmen kann entsprechend der Ausgangsposition justiert werden (Abb. 2.3.3).

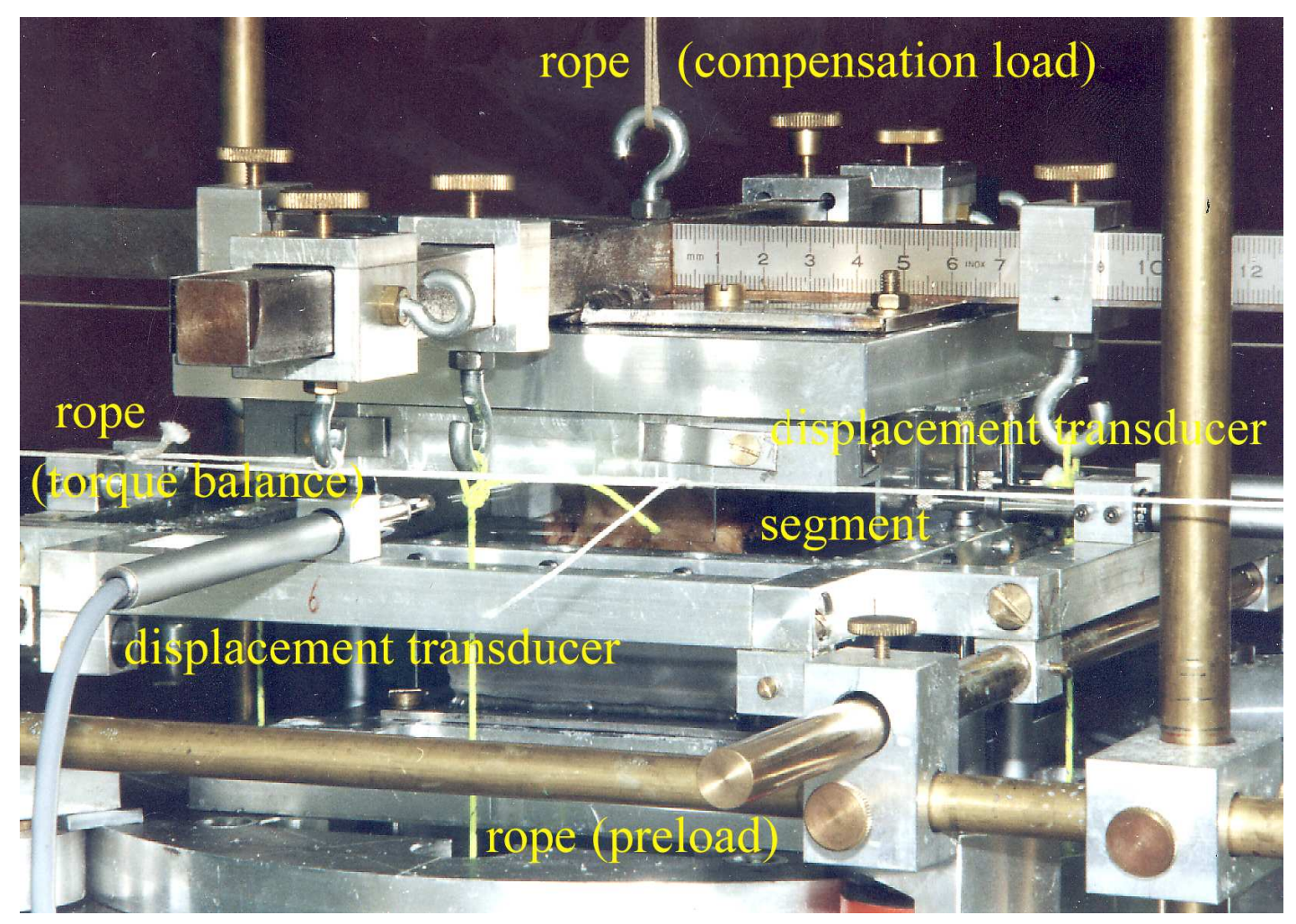

Abb. 2.3.3: Foto des Messsystems mit den Lagesensoren (displacement trancducer) und den Stahlwannen mit eingebettetem Brustwirbelsegment. Über ein Zugseil (rope) und Gegengewicht (compensation load) wird das Eigengewicht des Kraftkreuzes kompensiert. Ebenfalls abgebildet sind die Zugseile für die Drehmomentwaage (torque balance) und für die axiale Vorlast (preload). 


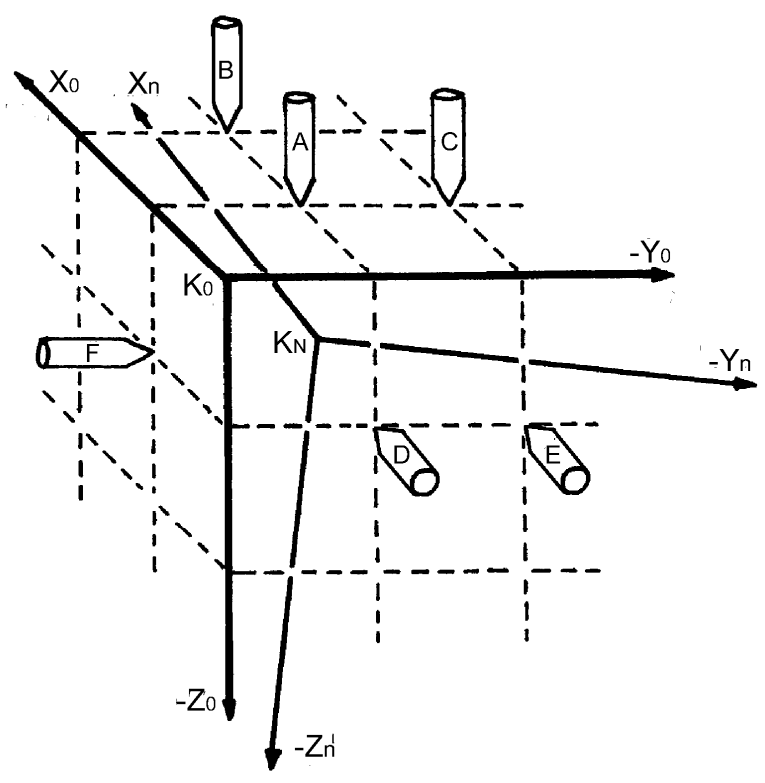

Abb. 2.3.4: Prinzip des Messwürfels. Schema der 3-2-1-Anordnung der Lagesensoren.

Das Ursprungskoordinatensystem ist $\mathrm{K}_{0}$ mit den normierten Achsen $\left(\mathrm{N}_{\mathrm{X} 0}\right.$,- $^{-}$ $\left.\mathrm{N}_{\mathrm{YO}},-\mathrm{N}_{\mathrm{ZO}}\right)$. Nach der Bewegung des Messobjekts wird das bewegte Koordinatensystem $K_{N}$ mit den normierten Achsen $\left(\mathrm{N}_{\mathrm{XN}},-\mathrm{N}_{\mathrm{YN}},-\mathrm{N}_{\mathrm{ZN}}\right)$ bestimmt, um den Translationsvektor des Koordinatenursprungs und den Rotationsvektor zu erhalten.

\subsubsection{Taster}

Bei dem verwendeten Messtaster (TYP $1310 \mathrm{Fa}$. Mahr) handelt es sich um einen

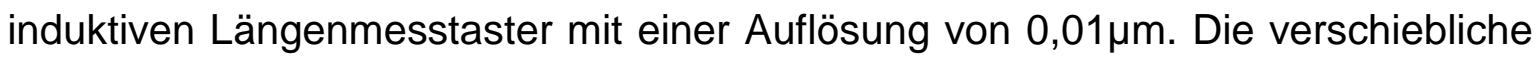
Tasterspitze wird von einer Feder kontinuierlich an die Messflächen gedrückt. Die Federkraft wurde soweit wie möglich reduziert, um eine Kraftübertragung und somit die Führung des Messobjekts durch die Federkraft zu minimieren. Die Bewegung von Tasterspitzen aus Stahl wird über eine Spule linear in Spannungswerte umgewandelt. Die Taster werden auf halbe Federkraft eingestellt, d.h. auf eine Mittelposition der Spannfeder, so dass eine Verkürzung der Tasterlänge (positive Auslenkung) und eine Verlängerung der Tasterlänge (negative Auslenkung) gleichermaßen möglich sind. Die verstärkten Spannungswerte von 4,9 $\mathrm{mV}$ pro Digitalisierungsstufe werden von Messinterface-System Millimar X1715 (Fa. Mahr) aufgenommen, in diskrete Werte umgewandelt und auf einem Personal Computer der Firma Agando eingelesen. Der Agando PC arbeitet mit einem Intel Core $^{\text {TM }} 2$ CPU-Chip und seine Leistung ist $1,87 \mathrm{GHz}$ mit 1,00 GB RAM. Auf dem PC ist die Software Millimar D 1000x installiert, die das Messinterface-System Millimar X1715 steuert und einrichtet. Die grafische Darstellung für den Hin- und Rücklauf in einem Messzyklus erfolgt durch ein mit LabView® (International Instruments) erstelltes Programm, so dass die gewonnene Daten weiter ausgewertet werden können. Eine Unterteilung des Signals in die 4097 Digitalisierungs- 
stufen („digits“) erlaubt eine detaillierte Auflösung der Bewegung von mindestens 0,4-96 digits/ $\mu \mathrm{m}$ (entsprechend 0,01-2,4 $\mu \mathrm{m}$ je nach Messbereich). Die Bewegungen des Messobjekts können nur eindimensional als Translation registriert werden. In der gewählten Tasteranordnung bei entsprechender Entfernung der Taster von der Rotationsachse bedeutet ein Drehwinkel $\alpha=1^{\circ}$ eine Verschiebung des Tastermesskopfs von mindestens $0,7 \mathrm{~mm}$ (290 digits; absolut 0.3 digits/mGrad). Die für die durchgeführten Messungen gewählte Aufnahmefrequenz ergibt zwischen 600-800 Messwerten pro Taster in einem Messzyklus.

Aus den Tasterrohdaten wird die tatsächliche Positionsveränderung des oberen Wirbelkörpers errechnet, in einer grafischen Form aufbereitet und mit der Anatomie korreliert.

\subsection{Die Kraftschraube}

Es wurde ein Koordinatensystem mit drei senkrechten Achsen (x-, y- und z-Achse) gewählt (Abb. 2.4.1). Durch die drei Achsen wird die Bandscheibe in drei Ebenen aufgespannt. Die mediane $x$-Achse und die transversale $y$-Achse spannen mittig in der Bandscheibe die Horizontalebene auf. Die x-Achse und die Longitudinalachse $Z$ bilden die Medianebene und die yz-Ebene stellt die Frontalebene dar. Jede beliebige Kraftschraube kann aus drei gerichteten Kräften $\underline{F}_{Z}, \underline{F}_{Y}, \underline{F}_{X}$ und drei Drehmomenten $T_{Z}, T_{Y}, T_{X}$ erzeugt werden.

Abbildung 2.4.1 zeigt exemplarisch eine mögliche Kraftschraube.

Es erfolgt eine gerichtete Kraftapplikation durch eine axiale Vorlast, entsprechend ihrer Wirkungsrichtung benannt $\underline{F}=\left(0,0, F_{Z}\right)=\underline{F}_{z}$.

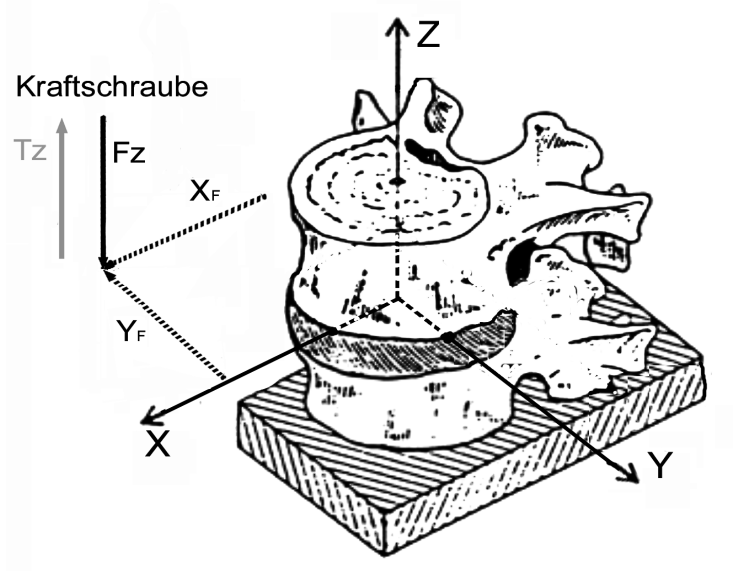

Abb. 2.4.1: Das gewählte Koordinatensystem und eine mögliche axiale Kraftschraube 
Das applizierte Drehmoment $T_{Z}$ für „Axialrotation“ ist parallel zur z-Achse ausgerichtet: $T(t)=\left(0,0, T_{Z}(t)\right)$. Entsprechend $T_{Y}$ für „Flexions-Extensions-Experimente“: $T(t)=\left(0, T_{Y}(t), 0\right)$ und für "Lateralflexion“ $T(t)=\left(T_{X}(t), 0,0\right)$.

Von den 6 Parametern der Kraftschraube (drei Kräfte und drei Drehmomente) bleiben innerhalb eines Experiments 5 Parameter konstant. Es wurde nur der Betrag und die Richtung des Drehmoments $\underline{I}=I T(\mathrm{t})$ I verändert. Der Kraftangriffspunkt (der Ortsvektor $\underline{F}_{z}\left(\mathrm{x}_{\mathrm{F}} / \mathrm{y}_{\mathrm{F}}\right)$ ), sowie die Kraftgröße (der Betrag IFI) variieren zwischen den Experimenten.

\subsubsection{Die axiale Kraftkomponente der Kraftschraube}

Die Aufbringung von axialen Vorlasten (Kraftvektor parallel zur z-Achse) erfolgt über aufgehängte Reiter (Abb. 2.4.2, 2.4.3). Die Bewegung ist statisch. Die Reiter sind parallel zur $x$ - Achse (sagittal) und $y$-Achse (frontal) des Koordinatensystems angeordnet und über Seile mit den jeweiligen Armen des Drehkreuzes verbunden. Die Führungsfreiheit ist wiederum durch die effektive Nutzung der langen Seile gegeben. Ein auf dem Reiter aufgehängtes Gewicht

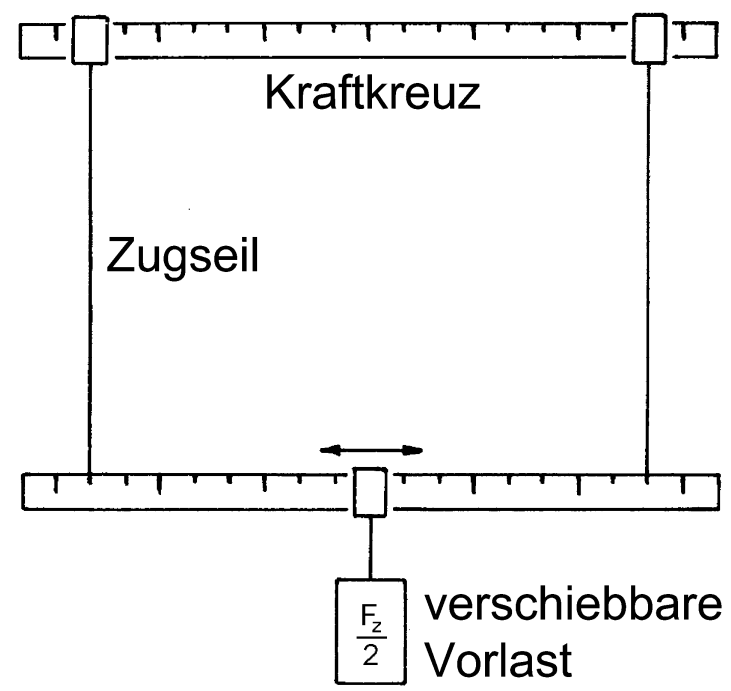

Abb. 2.4.2: Schema der Applikation der axialen Vorlast $F_{Z}$. kann entlang der Schiene so verschoben werden, dass je nach Positionierung des Reiters in sagittaler oder transversaler Richtung die verschiedenen Vorlastpositionen und Vorlastbeiträge erzielt werden können.

Das untersuchte Segment wird durch eine Gesamtvorlast von $50-400 \mathrm{~N}$ gleichermaßen auf beide Reiter verteilt kompressiv belastet.

Die Verschiebung des Reiters entlang der Führungsschiene erlaubt eine exakte Positionierung des Kraftvektors. Liegt der Kraftvektor nicht direkt im rotatorischen Widerstandszentrum des Bewegungssegments (beispielweise auf dem x-Reiter 
nach hinten oder nach vorne verlagert), so resultiert ein Kraftvektor der dorsal oder ventral der Bandscheibe zu liegen kommt. Die kompressive Kraft $F_{Z}$ erzeugt ein entsprechendes Drehmoment $T_{X}$ bzw. $T_{Y}$ und endet in einer Verkippung $\kappa_{\mathrm{x}}$ bzw. $\kappa_{\mathrm{y}}$ des oberen Wirbelkörpers. Diese Verkippung führt zu einer anderen Belastung in den Gelenken. Es kommt zu unter-schiedlichen Kontaktpunkten an den Gelenkflächen (NÄGERL ET AL. 1995). Dem zu Folge werden in dieser Studie die zyklische Drehmomentmessungen mit einer systematisch veränderten Position der axialen Vorlast als Basisparameter durchgeführt. Die axiale Vorlast simuliert zum einen das auf die Brustwirbelsäule kompressiv wirkende Gewicht

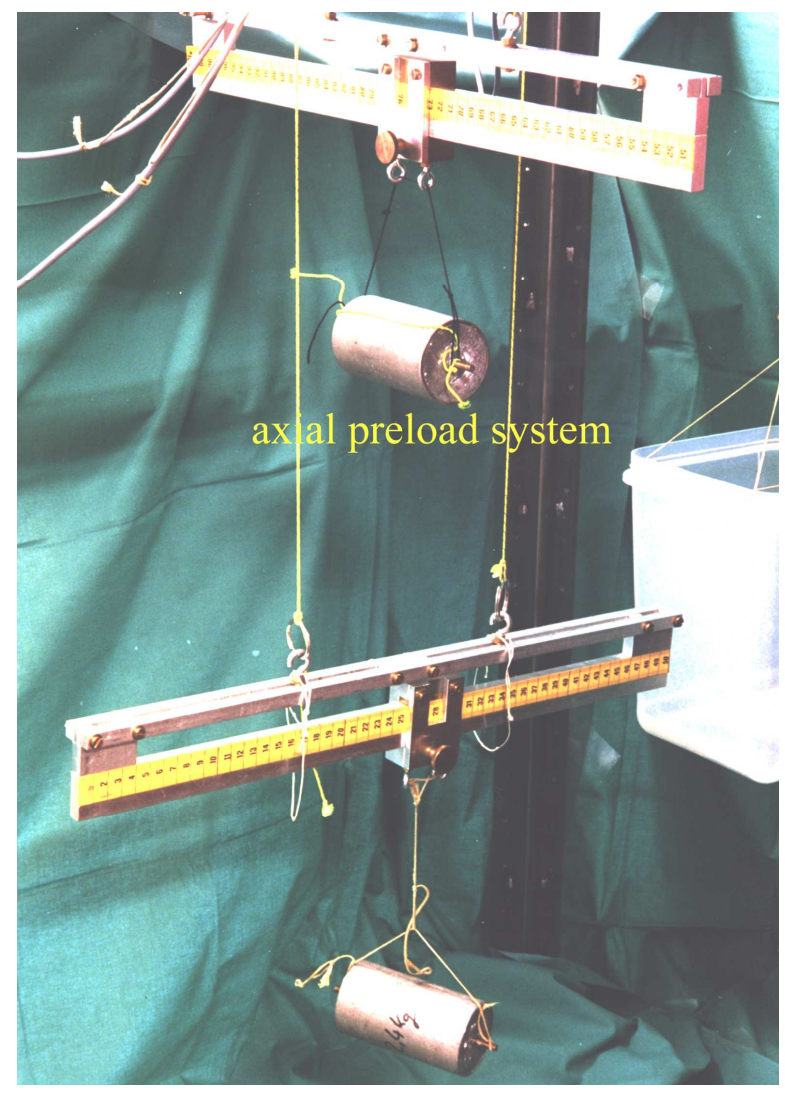

Abb. 2.4.3 Foto der Vorlastapplikation (axial preload system). Zwei gleichgroße Vorlasten werden über einen $x$ - und einen $y$-Reiter aufgebracht.

des Kopfes und des Oberkörpers, zum anderen ermöglicht sie durch das Anlegen des Kraftvektors $\left(\mathrm{X}_{\mathrm{F}} / \mathrm{y}_{\mathrm{F}}\right)$ in Verbindung mit dem aufgebrachten Drehmoment die Applikation verschiedener Kraftschrauben zur Simulation angreifender Muskelkräfte. 


\subsection{Die Applikation von Drehmoment}

In dem hier vorgestellten Aufbau wird das Konzept der Drehmomentwaage erläutert. Exemplarisch wird nur der Aufbau zur Erzeugung des Drehmoments $T_{Z}$ um die Longitudinalachse ausführlich erläutert.

Für das Erzeugen des Drehmoments werden Wassereimer verwendet. Beide Arme des Drehkreuzes sind in Neutralstellung parallel zur y-Achse ausgerichtet. An den vier zur x-Achse parallelen Seilen (in x-Richtung) werden über Umlenkrollen jeweils ein gleichschwerer Wasserbehälter $\mathbf{F}_{\mathrm{G}}$ aufgehängt (Abb. 2.5.1). Es greifen zunächst vier gleich große Kräfte an den y-Armen des Drehkreuzes, deren Kraftwirkungslinien zueinander parallel liegen. Die gegenüberliegenden Kräfte sind aber antiparallel, d.h. ihre Kraftvektoren heben sich auf. Man spricht von einer Drehmomentwaage. Das System befindet sich somit im Gleichgewicht. Die Anordnung ist kräftefrei.

Durch das Umpumpen einer bestimmten Wassermenge von einem Behälter in den anderen wird eine Kraft um einen bestimmten Betrag vermindert und die zweite gleichgerichtete Kraft um den gleichen Betrag erhöht. Die Summe aller Kräfte bleibt aber auf jeder Seite des y-Armes des Drehkreuzes gleich groß. Berechnet man jedoch die Bilanz der zur x-Achse parallel gerichteten Kräfte, so ist jeweils eine Differenzkraft übrig. Die beiden Differenzkräfte sind gleichgroß und entgegengesetzt, so dass sie sich kompensieren und anbei ein reines Drehmoment $\mathrm{T}(\mathrm{t})$ erzeugen.

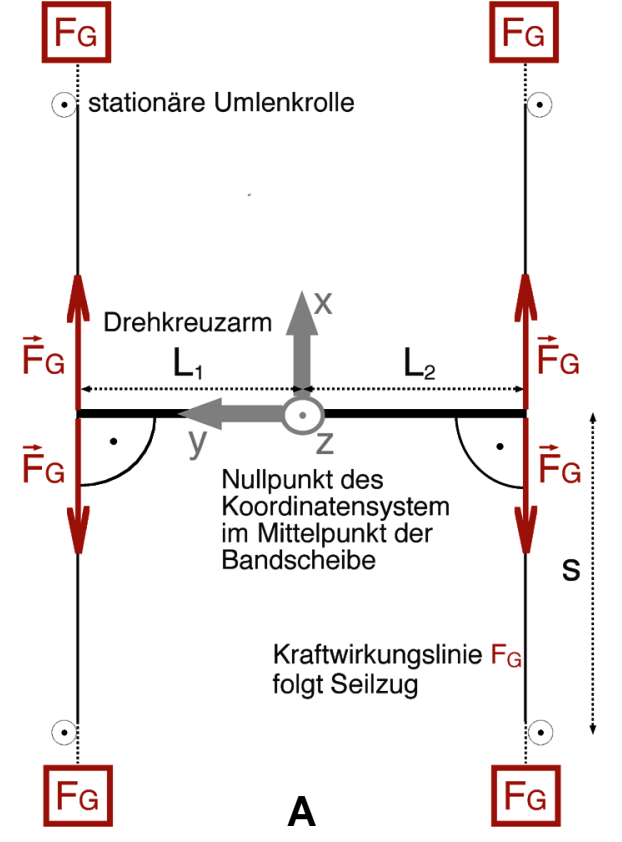

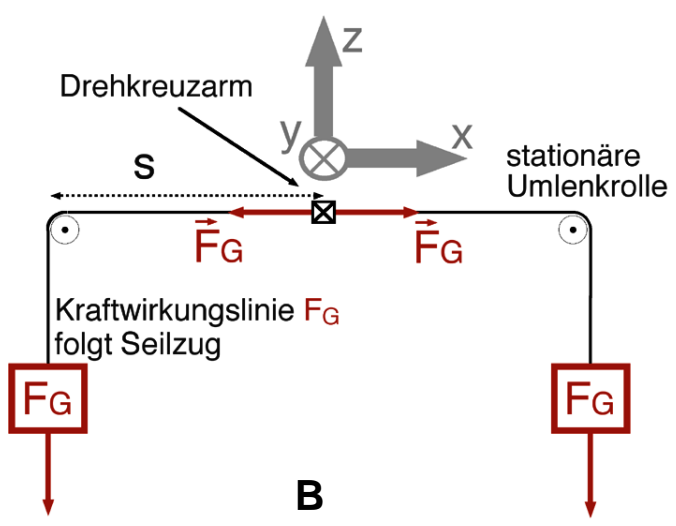

Abb. 2.5.1: Aufbau einer Drehmomentwaage bei Applikation eines Drehmoments $T_{Z}$ von oben (A) und in Ansicht von rechts (B). (MANSOUR 2001) 
Das Pumpsystem wird dorsal des in Apparatur eingespannten Bewegungssegments angebracht.

Bei einer Umlagerung des gesamten Wasservolumens von einem Behälter in den anderen und zurück spricht man von einem Messzyklus.
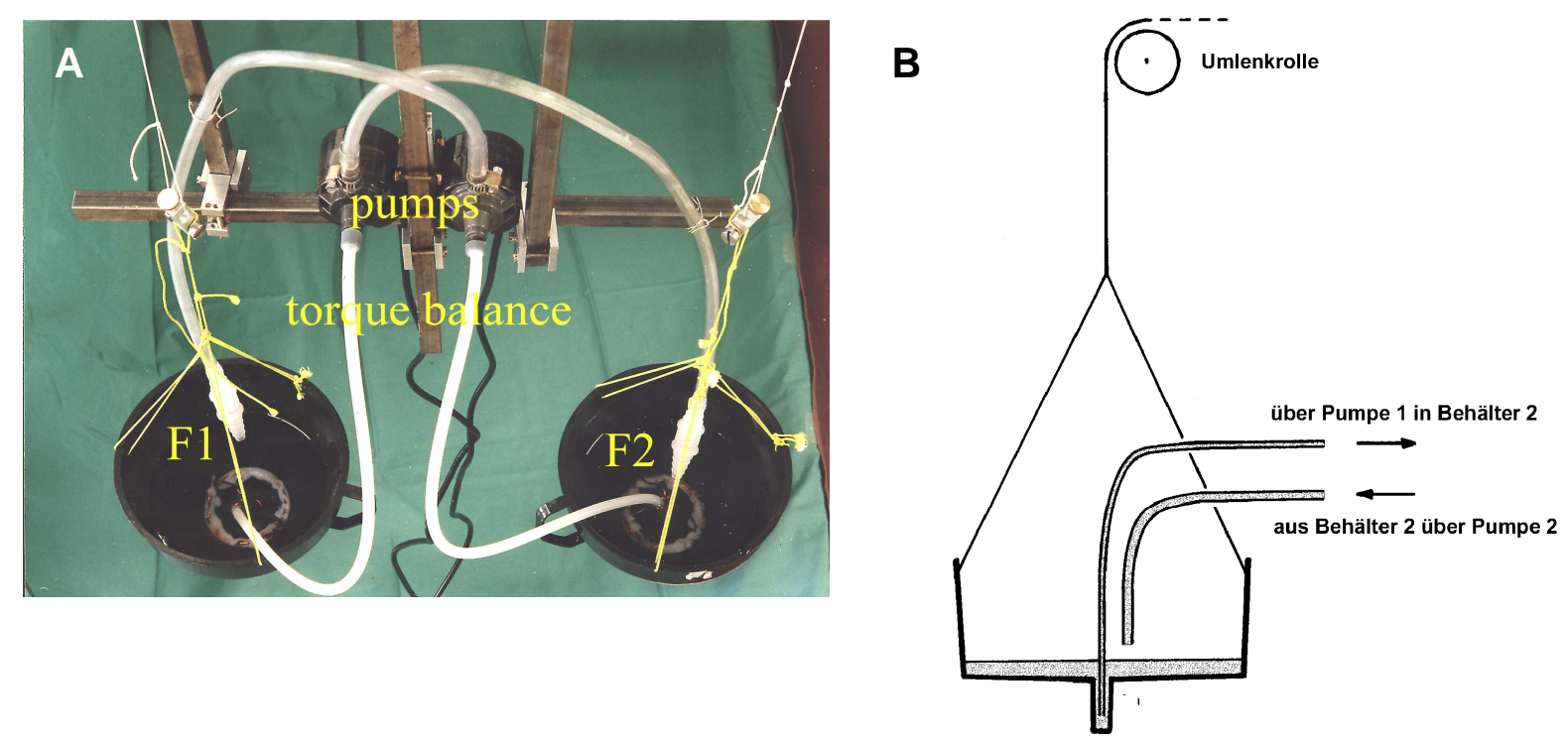

Abb. 2.5.2: Realisation der Drehmomentwaage; Foto (A) das Pumpsystem. Schema (B) das Leitungssystem eines Wasserbehälters. Für größere Wassermengen wurden entsprechende Behälter benutzt.

Die verwendeten Pumpen werden luftfrei befüllt. Das Leerlaufen der Pumpen wird durch zwei Rückschlagventile (Fa. Schütt) und eine Restwassermenge im Behälter von $1500 \mathrm{ml}$ verhindert. Für den Aufbau der Drehmomentwaage werden die Pumpen Eheim 8060 mit einer Fördermenge von 4,5 I/ min verwendet. In die Wasserbehälter sind zwei Elektroden eingebaut, die mittels eines elektrischen Testsignals den Befüllungsstand überwachen. Wird der Widerstand zwischen zwei Elektroden erhöht (das passiert, wenn eine Elektrode über Wasser hängt und kein Strom mehr fließt) und das Testsignal unterbrochen, schaltet das Kontrollgerät die Pumprichtung um. So überwacht ein Kontrollgerät den Wechsel der Pumprichtung und sorgt für eine zeitliche Abstimmung.

Für die Applikation eines Drehmoments $T_{X}$ bzw. $T_{Y}$ parallel zur x-Achse bzw. y-Achse ist der Aufbau ähnlich (Abb. 2.5.3, S. 59). 
Bei Applikation eines Drehmoments $T_{x}$ werden die Wasserbehälter mit angeschlossenen Pumpen an den y-Armen des Drehkreuzes angebracht. Die Gegengewichte sind dann so über die Umlenkrollen aufgehängt, dass der Kraftvektor entgegengesetzt ist und somit die Gewichtkraft $F_{G}$ des genannten Behälters kompensiert. Die Anordnung ist auch hier kräftefrei.

Bei $T_{Y}$ hängen die Wasserbehälter, deren Inhalt umgepumpt wird, an den $\mathrm{x}$-Armen des Drehkreuzes. Ein Gegengewicht kompensiert die Gewichtskraft der Wasserbehälter.
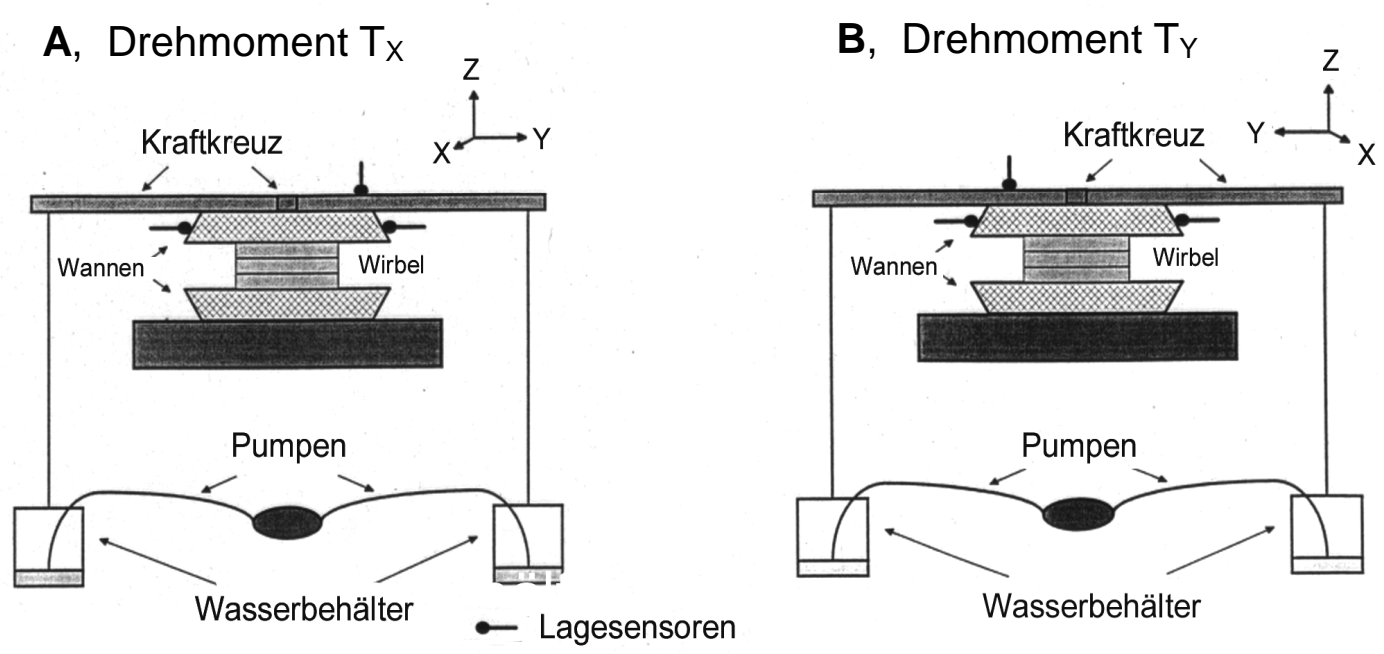

Abb. 2.5.3: Applikation eines Drehmoments $T_{X}(\mathbf{A})$ oder $T_{Y}(\mathbf{B})$.

\subsubsection{Prüfung des Umwälzsystems}

Des Weiteren wurde die Kontinuität der Umpumpgeschwindigkeit in einem Zyklus unter den gleichen Versuchsbedingungen für beide Pumpensätze überprüft. Eine Wassermenge von $5 \mathrm{l}$ wurde in ein Messbehältnis umgepumpt und alle $500 \mathrm{ml}$ die ver-strichene Zeit notiert. Es wurden 5 Messreihen pro Pumpe durchgeführt und ein Mittelwert ermittelt. In Abbildung 2.5.4 A, B (S. 60) repräsentieren die Datenpunkte den Durchschnittswert von diesen 5 Messzyklen. Die Balken geben den Standardfehler wieder. Lineare Regression ergab einen Korrelationskoeffizienten $r$ $=0,999 ; p<0,0002$ und $r=0,999 ; p<0,0001$ für die Leni 30-Pumpen und mit $p=$ 0,89 zeigte sich kein signifikanter Unterschied der beiden Pumpen zueinander. Für die Eheim 8060-Pumpen beträgt $r=0,999 ; p<0,00002$ und $r=0,999 ; p<0,0001$ 
und $p=0,94$. Die Messergebnisse unterschieden sich nicht signifikant von einer linearen Funktion $\mathrm{t}(\mathrm{V})$, damit arbeiten die Pumpen hinreichend proportional.

Ein weiteres Experiment bestätigte die konstante Pumpzeit in aufeinander folgenden Umwälzzyklen $t(n)$ (Abb. 2.5.5 A, B). Nach dem erfolgten Umpumpen des Wasservolumens $5 \mathrm{l}$ (Leni 30-Pumpen bzw. Eheim 8060-Pumpen) wurde die Umpumpzeit notiert. Die Umpumpzeit blieb für beide Pumpen annähernd konstant. Für die $t(n)$-Funktion gilt es $p=0,996-0,997$ für die Leni 30-Pumpen und 0,9990,997 für die Eheim 8060-Pumpen. Die beiden jeweiligen Pumpen unterscheiden sich in Ihrem Verhalten $(p<0,01)$ unwesentlich.
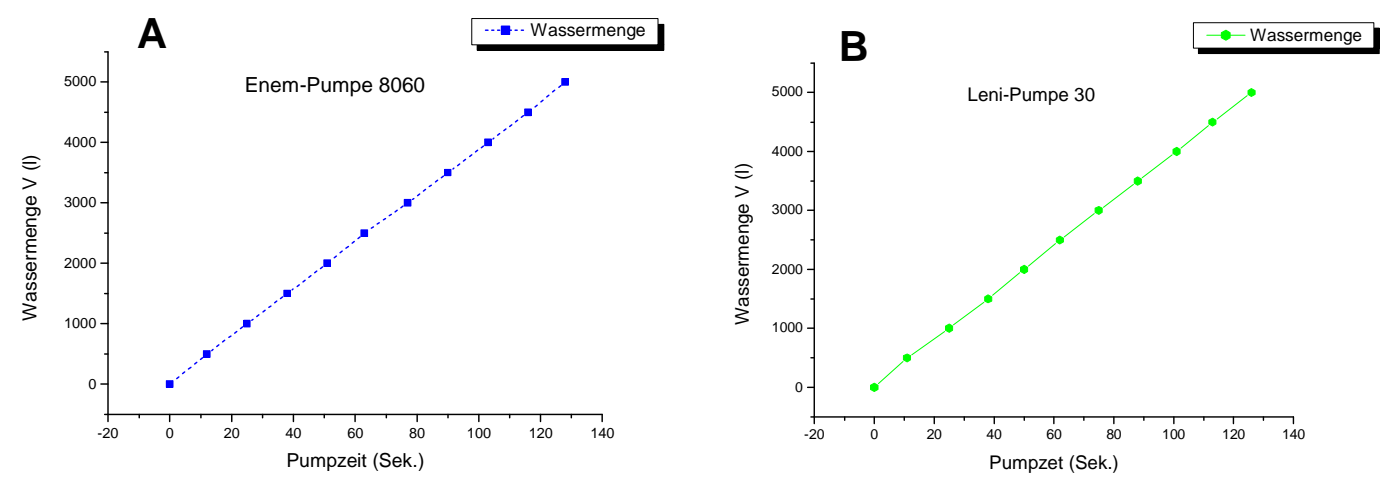

Abb. 2.5.4: Linearität der Pumpleistung $t(\mathrm{~V})$ während eines Messzyklus der Eheim 8060 (A) und der Leni 30- Pumpen (B).
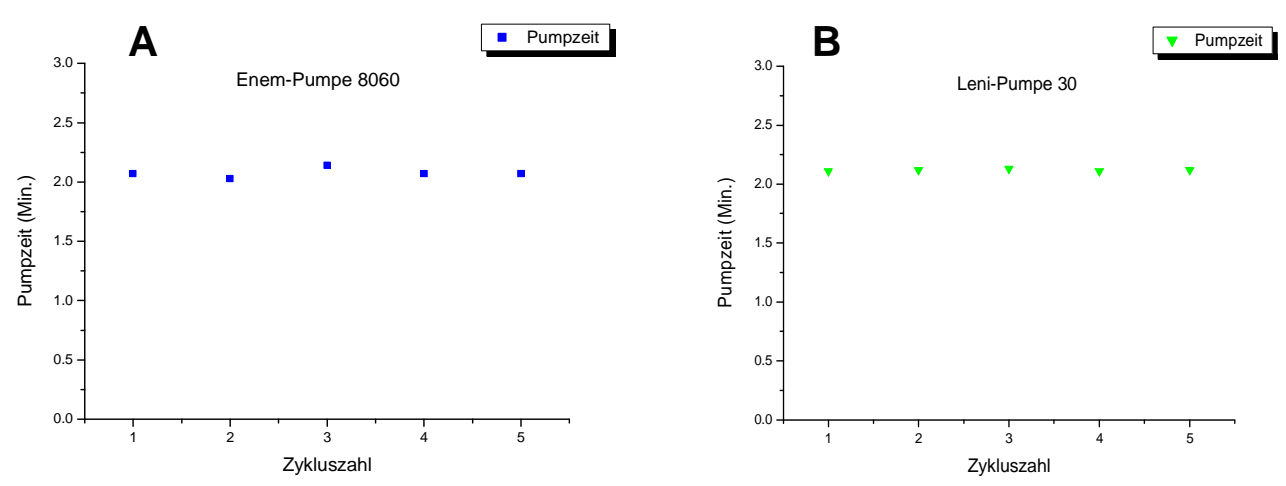

Abb. 2.5.5: Konstante Pumpdauer der Eheim 8060 (A) und der Leni 30- Pumpen (B) 


\section{Linearität des Drehmoments in Drehmomentwaage}

Ein reines Drehmoment wird erzeugt, indem die in Extrempositionen des gedrehten Drehkreuzes entstehen Zugkräfte von den Gegenkräften kompensiert werden. Durch den großen Abstand (mind. 0,5 m) zwischen dem Drehkreuz und der Umlenkrolle wird die Führung des Messobjekts beim Auslenken durch die Seilzüge verhindert.

Das linear ansteigende Drehmoment wird über einen Seilzug der Länge $\mathbf{s}(0,5 \mathrm{~m})$ mit Hilfe einer Umlenkrolle zwischen dem Endpunkt des Stahldrehkreuzes und dem Wasserbehälter ermittelt. Wenn sich das Stahlkreuz dreht, ändert sich die Seitenlänge s, und somit auch die Richtung des Seils, also die Richtung der Kraft $\underline{F}_{G}$. Diese Auslenkung verändert den Winkel zwischen Hebel $L$ und der Kraft $\underline{F}$ um $\alpha^{*}$. Die Kraft $\underline{F}_{G}$ verändert ihre Richtung um $\beta$. Der Betrag von $\underline{F}_{G}$ bleibt bei reibungsfreier Anordnung konstant (Abb. 2.5.6). Die Länge des Seilzugs zur Rolle verkürzt sich um $s_{1}$ auf $s_{2}$.

Es gilt also für Drehmoment $\underline{T}: \underline{I}=\underline{F}_{G} \times \underline{L}$ und $T=F G \bullet L \bullet \sin \chi\left(\sin 90^{\circ}=1\right)$.

Für die Größe des Drehmoments ist die auf dem Hebelarm L senkrecht stehende Komponente $\underline{\mathrm{F}}_{\mathrm{G}}$ von Bedeutung. Der zu $\underline{\mathrm{L}}$ gleichgerichteter Anteil erzeugt eine Lagerkraft $\left(\underline{F_{L}}\right)$. In einer reinen Drehmomentwaage wird diese Kraft durch eine entgegengesetzte Kraft $-\underline{F_{L}}$ neutralisiert. Eine exakte Bestimmung von $\mathrm{F}_{\mathrm{T}}$ und $\mathrm{F}_{\mathrm{L}}$ wird in Abbildung 2.5.6 gezeigt. Der Hebelarm des Drehkreuzes L, die Zugseillänge $s$ und der Drehwinkel $\alpha$ sind bekannt.

Die trigonometrischen Zusammenhänge sind wie folgt:

$$
\begin{aligned}
& b=2{ }^{*} L^{*} \sin ^{-1}(\alpha / 2) \\
& \chi_{1}=(180-\alpha) / 2 \\
& \chi_{2}=90^{\circ}-\chi_{1}
\end{aligned}
$$

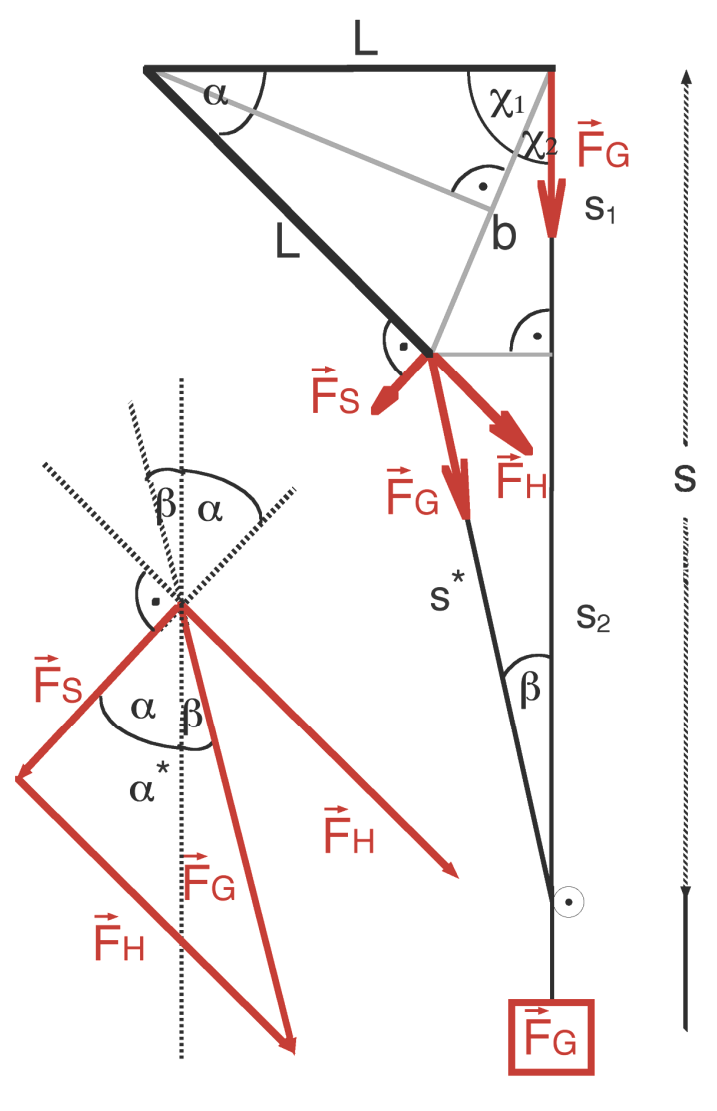

Abb. 2.5.6: Fehlerabschätzung bei der Erzeugung eines Drehmoments 


$$
\begin{aligned}
& d=b * \sin \chi_{2} \\
& s_{1}=b * \cos \chi_{2} \\
& s_{2}=s-s_{1} \\
& \tan \beta=d / s_{2} \\
& \alpha^{*}=\alpha+\beta \\
& F_{S}=F_{G}{ }^{*} \cos \alpha^{*} \\
& \underline{F}_{H}=F_{G}{ }^{*} \sin \alpha^{*}
\end{aligned}
$$

Es gilt also:

$$
\text { für } \mathrm{s}^{*} \cong \mathrm{s} \cong \mathrm{s}_{2} \Rightarrow \beta \rightarrow 0 \wedge \alpha^{*} \cong \alpha(\mathrm{s}=50 \mathrm{~cm}) ; \Rightarrow \mathrm{F}_{\mathrm{T}} \| \mathrm{F}_{\mathrm{G}} \text {. }
$$

Für die maximale Auslenkung des Th2/3-Segments von $\pm 6^{\circ}$ bedeutet die folgende Fehlerabschätzung

$$
\begin{aligned}
& \mathrm{F}_{\mathrm{T}}=\mathrm{F}_{\mathrm{G}}{ }^{*} \cos 6^{\circ} \\
& \mathrm{F}_{\mathrm{T}}=\mathrm{F}_{\mathrm{G}}{ }^{*} 0,995 \\
& \Rightarrow \mathrm{F}_{\mathrm{T}} \cong \mathrm{F}_{\mathrm{G}} .
\end{aligned}
$$

\subsection{Analyse der Positionsveränderungen}

Wie in Kapitel 1.3.2 (S. 22) beschrieben hat die Lage eines starren Körpers im Raum sechs Freiheitsgrade. So benötigt man zur Beschreibung seiner Bewegung sechs unabhängige Lagersensoren, wie es für die in dieser Studie verwendete Messapparatur der Fall ist. Die Taster A, B, C (xy-Ebene) registrieren eine Lageveränderung entlang der z-Achse des Ursprungskoordinatensystems, die Taster $D, E$ (yz-Ebene) entlang der x-Achse, und der Taster $F$ (xz-Ebene) nimmt die Translation entlang der y-Achse auf. Mit nur sechs Lagersensoren können die drei Ebenen (xy, xz und yz) aufgespannt durch die drei Koordinatenachsen nun exakt bestimmt werden. Mit der Bestimmung dieser drei Ebenen (xy, xz, yz) kann das bewegte Koordinatensystem $\mathrm{K}_{\mathrm{n}}$ errechnet und in Relation zu dem Ausgangskoordinatensystem $\mathrm{K}_{0}$ gesetzt werden.

Der fest in Apparatur angebundene untere Wirbelkörper repräsentiert das Ausgangskoordinatensystem Ko. Mit dem bewegten oberen Wirbelkörper ist ein zweites Koordinatensystem $\mathrm{K}_{\mathrm{n}}$ starr verbunden. Eine Bewegung des oberen Wirbel- 
körpers wird von den Lagersensoren registriert. Aus den eingelesenen Positionsangaben wird dann die neue Position des Messobjekts im Raum $\left(K_{n}\right)$ in Relation zur Referenzsystem (Ko) bestimmt. Die Positionsveränderung lässt sich dann nach TEICHMANN (1973) in eine Verschraubung umrechnen.

\subsubsection{Analyse der Tastermesswerte}

Man nimmt die nacheinander eingenommenen Positionen des Messobjekts als Funktion der Zeit ( $\mathrm{t}$ ) an. Die drei Ebenen des bewegten Koordinatensystems zum Zeitpunkt $t_{0}$ werden festgelegt und fallen mit drei Ausgangsebenen ( $x y, x z$, und yz) im Referenzkoordinatensystem $\mathrm{K}_{0}$ zusammen. Hierzu werden die Messtaster A, B, C, D, E und F in der „3-2-1“ Anordnung genutzt (Abb. 2.6.1). Die neue Position des Messobjekts mit dem bewegten Koordinatensystem $K_{n}$ zum Zeitpunkt $t_{n}$ berechnet sich aus den veränderten Tasterwerten A-F. Die Position von $K_{n}$ ist bestimmt durch einen Translationsvektor $\underline{d}_{0}$ : Ursprung von $\mathrm{K}_{0}$ zum Nullpunkt von $\mathrm{K}_{\mathrm{n}}$. Die Rotation ergibt sich aus dem Rotationsvektor $\underline{\mu}\left(\mu_{\mathrm{x}}, \mu_{\mathrm{Y}}, \mu_{\mathrm{z}}\right)$. Zur genauen räumlichen und zeitlichen Auflösung wird jeweils die Lageveränderung des momentanen Koordinatensystems $K_{n}$ relativ zum vorausgegangenen Koordinatensystem $\mathrm{K}_{\mathrm{n}-1}$ und absolut zum Ursprungskoordinatensystem $\mathrm{K}_{0}$ bestimmt. Die Positionsveränderung des Koordinatensystems ist zur Analyse der Bewegungsstruktur aber zu ungenau. Nach dem „Konzept der wandernden Schraubachsen“ von GOLDSTEIN (1983) wird die differentiell kleine Bewegung des Koordinatensystems $K_{n}$ relativ zu $K_{n-1}$ als eine Schraube um eine momentane Schraubachse $d_{n}(\alpha)(v g l$. Kap 1.3.2, S. 22) angesehen.

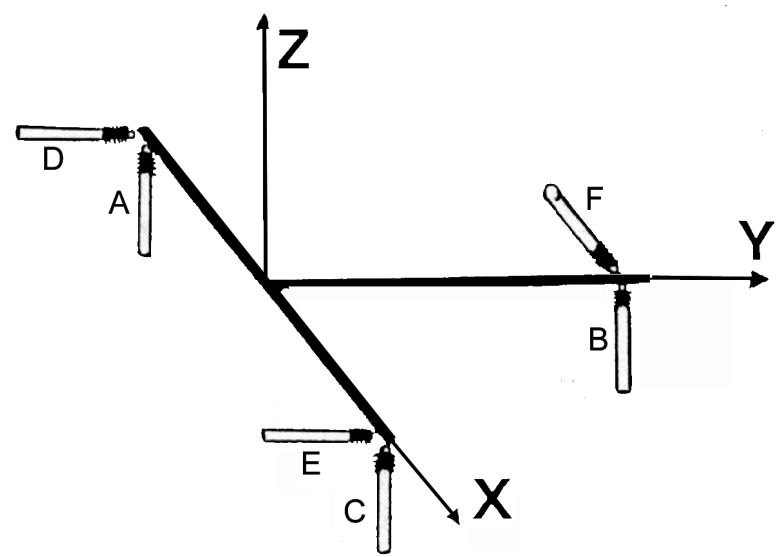

Abb. 2.6.1: Schema der Anordnung der Messtaster A-F. 


\subsubsection{Berechnung des Ausgangs- und bewegten Koordinatensystems}

Die Ausgangslage des Wirbelkörpers wird mit Hilfe von Ortsvektoren des Tasterkontaktpunktes mit der Glasplatte (Tasterortsvektoren) $\underline{A}\left(x_{a}, y_{a}, a\right), \underline{B}\left(x_{b}, y_{b}, b\right)$, $\underline{\mathrm{C}}\left(\mathrm{x}_{c}, \mathrm{y}_{c}, \mathrm{c}\right), \underline{\mathrm{D}}\left(\mathrm{d}, \mathrm{y}_{\mathrm{d}}, \mathrm{z}_{\mathrm{d}}\right), \underline{\mathrm{E}}\left(\mathrm{e}, \mathrm{y}_{\mathrm{e}}, \mathrm{z}_{\mathrm{e}}\right), \underline{\mathrm{F}}\left(\mathrm{x}_{\mathrm{f}}, \mathrm{f}, \mathrm{z}_{\mathrm{f}}\right)$ definiert. Die Koordinaten $\mathrm{x}_{\mathrm{i}}, \mathrm{y}_{\mathrm{i}}, \mathrm{z}_{\mathrm{i}}$ sind bekannt und konstant. Die variablen Koordinaten a bis $f$ eines jeweiligen Tasterortsvektors entsprechen dem Abstand vom Nullpunkt des Koordinatensystems und werden kontinuierlich eingelesen.

Zum Zeitpunkt $t_{0}$ :

Die beiden durch Ebene $\underline{X Y}_{0}$ aufgespannten Vektoren verrechnen sich wie folgt $\underline{\mathrm{V}}_{1}=\underline{\mathrm{A}}-\underline{\mathrm{B}}$ und $\underline{\mathrm{V}}_{2}=\underline{\mathrm{A}}-\underline{\mathrm{C}}$.

Der Richtungsvektor der Z-Achse $\underline{N} z 0=(\underline{A}-\underline{B}) \times(\underline{A}-\underline{C})$.

Die zweite Ebene $\underline{Y Z}_{0}$ wird definiert durch die Vektoren $\underline{\mathrm{V}}_{3}=\underline{\mathrm{V}}_{1} \times \underline{\mathrm{V}}_{2}\left(\underline{\mathrm{V}}_{3} \perp \underline{\mathrm{V}}_{1} \wedge \underline{\mathrm{V}}_{3} \perp \underline{\mathrm{V}}_{2}\right.$, Ergebnis des Kreuzprodukts) und $\underline{\mathrm{V}}_{4}=\underline{\mathrm{D}}-\underline{\mathrm{E}}$.

Der Richtungsvektor der x-Achse $\underline{N}_{x 0}=\underline{N}_{z 0} \times(\underline{D}-\underline{E})$.

Zur Bestimmung der Ebene $\underline{X Z}_{0}$ benötigt man $\underline{V}_{5}=\underline{V}_{3} \times \underline{V}_{4}$.

Der Richtungsvektor der Y-Achse $\underline{\mathrm{N}}_{\mathrm{Y} O}=\underline{\mathrm{N}}_{\mathrm{ZO}} \times \underline{\mathrm{N}}_{\mathrm{X} 0}$.

Der Schnittpunkt der drei Ebenen $\underline{X Y_{0}}, \underline{X Z}_{0}, \underline{Y Z}_{0}$ definiert den Nullpunkt $K_{0}\left(X_{0}, y_{0}\right.$, $\left.\mathrm{Z}_{0}\right)$ des Koordinatensystems $\underline{\mathrm{K}}_{0}$ mit den Achsenvektoren $\underline{\mathrm{N}} \mathrm{x}_{0}, \underline{\mathrm{N}} \underline{Y}_{0}, \underline{\mathrm{N}} \mathrm{z}_{0}\left(\underline{\mathrm{X}}_{0}, \underline{\mathrm{Y}}_{0}, \underline{Z}_{0}\right.$; die normierten Achsenvektoren des Koordinatensystems).

Beim Ansetzen der Bewegung ändern sich die Tasterortsvektoren: $\underline{A}_{n}\left(x_{a}, y_{a}, a+\Delta a\right)$, $\underline{B}_{n}\left(x_{b}, y_{b}, b+\Delta b\right), \underline{C}_{n}\left(x_{c}, y_{c}, c+\Delta c\right), \underline{D}_{n}\left(d+\Delta d, y_{d}, z\right), \underline{E}_{n}\left(e+\Delta e, y_{e}, z_{e}\right), \underline{F}_{n}\left(x_{f}, f+\Delta f, z_{f}\right)$. So kann die Bestimmung von $\underline{X Y}, \underline{Y Z_{n}}$ und $\underline{X Z_{n}}$ des Koordinatensystems $K_{n}$ Zum Zeitpunkt $t_{n}$ mit dem Ursprung $\underline{k}_{n}$ und den normierten Achsen $\underline{X}_{n}, \underline{Y}_{n}, \underline{Z}_{n}$ mit Hilfe von veränderten Tasterortsvektoren $\underline{A}_{n}, \underline{B}_{n}, \ldots \underline{E}_{n}$ erfolgen.

Die Koordinaten des Schnittpunktvektors $\underline{k}_{n}\left(k_{x n}, k_{y n}, k_{z n}\right)$ sind die Abstände $z u$ den Achsen des Ausgangskoordinatensystems. Die Projektion der Tastervektoren $\underline{A}_{n}, . ., \underline{F}_{n}$ auf die normierten Achsenvektoren $\underline{X}_{n}, \underline{Y}_{n}, \underline{Z}_{n}$ bestimmen die Koordinaten von $\underline{d}_{n}$. 
$\mathrm{k}_{\mathrm{xn}}=\underline{\mathrm{D}}_{\mathrm{n}} \cdot \underline{\mathrm{X}}_{\mathrm{n}}=\underline{\mathrm{E}}_{\mathrm{n}} \cdot \underline{\mathrm{X}}_{\mathrm{n}}$

$\mathrm{k}_{\mathrm{yn}}=\underline{\mathrm{F}}_{\mathrm{n}} \cdot \underline{\mathrm{Y}}_{\mathrm{n}}$

$\mathrm{k}_{\mathrm{zn}}=\underline{\mathrm{A}}_{\mathrm{n}} \bullet \underline{\mathrm{Z}}_{\mathrm{n}}=\underline{B}_{\mathrm{n}} \bullet \underline{Z}_{\mathrm{n}}=\underline{\mathrm{C}}_{\mathrm{n}} \bullet \underline{\mathrm{Z}}_{\mathrm{n}}$.

Der Schnittpunktvektor $\underline{k}_{n}$ kann also als Linearkombination der Normalenvektoren in Bezug auf das Ausgangskoordinatensystem $\mathrm{K}_{0}$ dargestellt werden:

$\underline{\mathrm{k}}_{\mathrm{n}}=\mathrm{k}_{\mathrm{x}} \cdot \underline{\mathrm{X}}_{\mathrm{n}}+\mathrm{k}_{\mathrm{y}} \cdot \underline{\mathrm{Y}}_{\mathrm{n}}+\mathrm{k}_{\mathrm{z}} \cdot \underline{\mathrm{Z}}_{\mathrm{n}}$

Der differentielle Translationsvektor d zwischen den Koordinatensystemen $K_{n-1}$ und $K_{n}$ ergibt sich aus:

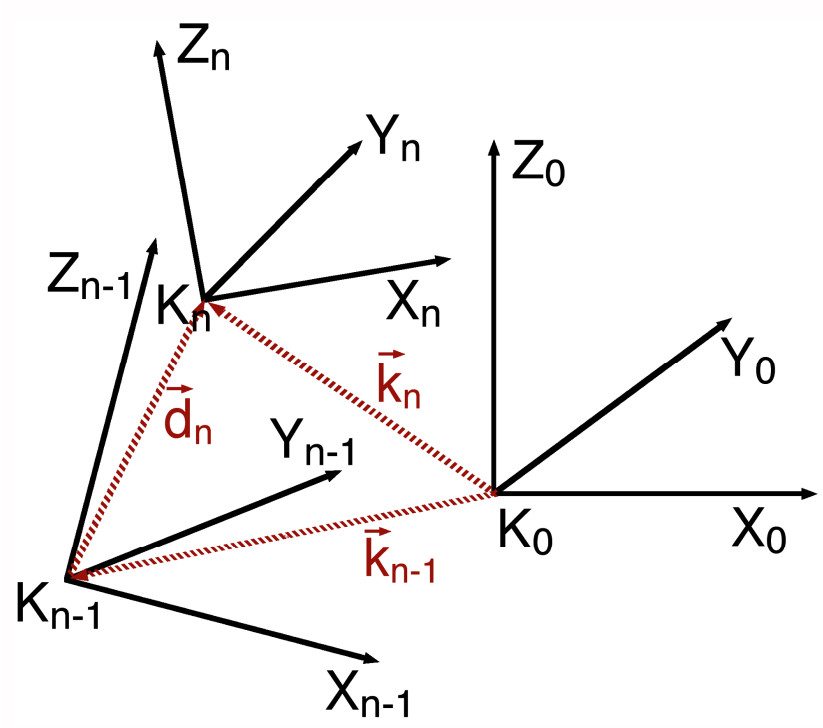

Abb. 2.6.2: Bestimmung des Verschiebevektors. $\underline{\mathrm{d}}=\underline{\mathrm{k}}_{n}-\underline{\mathrm{k}}_{n-1}$ (Abb. 2.6.2).

Das System kann sich auch drehen. So berechnet man zusätzlich ein Rotationsvektor oder auch Drehvektor genannt.

Der Drehvektor $\mu_{n}\left(\mu_{x n}, \mu_{y n} \mu_{z n}\right)$ beschreibt eine Rotation des momentanen Koordinatensystems $\mathrm{K}_{n}$ gegen das Ausgangskoordinatensystem $\mathrm{K}_{0}$ (Abb. 2.6.3).

Folgend errechnen sich die Rotationskomponenten:

$\tan \mu_{\mathrm{xn}}=\left(\Delta \mathrm{z}_{\mathrm{c}}-\Delta \mathrm{z}_{\mathrm{a}}\right) /\left(\mathrm{y}_{\mathrm{c}}-\mathrm{y}_{\mathrm{a}}\right)$

$\tan \mu_{\mathrm{yn}}=\left(\Delta \mathrm{z}_{\mathrm{b}}-\Delta \mathrm{z}_{\mathrm{a}}\right) /\left(\mathrm{y}_{\mathrm{a}}-\mathrm{y}_{\mathrm{b}}\right)$

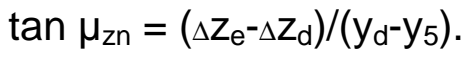

Die Definition des Vorzeichens der Parameter im mathematisch positiven Sinne erfolgt im rechtshändigen Koordinatensystem.

Vergleicht man die Einheitsvektoren des

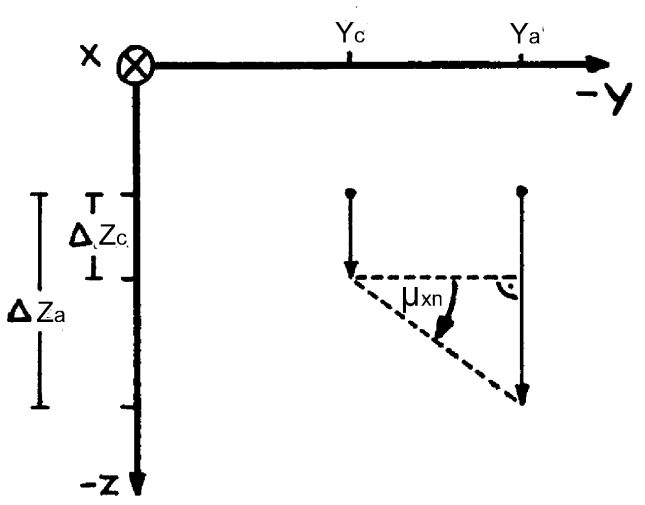
Ausgangs- und des bewegten Koordinatensystems lässt sich eine Drehmatrix erstellen. Durch Hauptachsentransformation

Abb. 2.6.3:Trigonometrische Beziehung zur Berechnung der $\mu_{x}-$ Komponente des Drehvektors $\mu$. berechnet sich dann der Drehvektor. 


\subsubsection{Berechnung der Verschraubungsparameter}

Der Rotationsvektor $\underline{\mu}$ bestimmt zugleich den Richtungsvektor der Schraubachse. Die Normierung liefert den Einheitsvektor $\underline{\mathrm{e}}=1 / \underline{\underline{\mu}} \mathbf{l}\left(\mu_{\mathrm{x}}, \mu_{\mathrm{y}}, \mu_{\mathrm{z}}\right)$.

Der Betrag des Drehvektors $\underline{\mu}$ ist der Rotationswinkel $\mu$ um die Schraubachse. Der Winkel $\phi$ ist die Verkippung des Richtungsvektors in Bezug auf das Ursprungskoordinatensystem. Der Versatz $s$ entlang der Schraubachse wird folgendermaßen berechnet: $\mathrm{s}=\underline{\mathrm{d}} \bullet \underline{\mathrm{e}}$.

Die Schraubsteigung $\tau$ bezogen auf $\mu$ ist $\tau=s / \mu=\underline{d} \bullet \underline{\mu} /|\underline{\mu}|^{2}$.

Nach TeICHMAnN (1973) ergibt sich der Ortsvektor a (Aufpunktvektor) der Schraubachsgeraden mit dem kürzesten Abstand a zum Ursprung des Koordinatensystems wie folgt: $\underline{a}=0,5 \bullet[\underline{d}-\mathrm{s} \bullet \underline{e}+\cot \mu / 2 \bullet(\underline{e} \times \underline{d})]$.

Nach diesem Verfahren wurden die Schraubachsen $r_{i}$ zum einem in Bezug auf das Ursprungskoordinatensystem $\mathrm{K}_{0}$ ermittelt, wodurch der absolute Drehwinkel $\alpha$ des oberen Wirbelkörpers berechnet wurde $(\mu=\alpha)$. Zum anderem in Bezug auf das bewegte Koordinatensystem $\mathrm{K}_{\mathrm{n}-1}$, um den differentiellen Rotationswinkel $\mathrm{d} \mu$, den Versatz ds und die Schraubsteigungen $\mathrm{d} \tau$ zu bestimmen. Die Durchstoßpunkte $R_{i}=\left(x_{i}, y_{i}, z_{i}\right)$ differentieller Schraubachsen $d r_{i}(\alpha)$ wurden in Bezug auf das Ausgangskoordinatensystem dargestellt.

Die Akquisitionsfrequenz der Tastermesswerte bestimmt die Anzahl der berechneten differentiellen Schraubachsen. Die Frequenz muss so hoch sein, um eine nahezu lückenlöse Abdeckung der Bewegungsstruktur zu erreichen. Ein Drehwinkel $\mathrm{d} \mu$ von $0,01 \mathrm{~m}^{\circ}$ um die momentane Schrauba chse wurde als differentiell klein betrachtet. Es muss also von Achsen ausgegangen werden, die über diesen kleinen Bereich gemittelt wurden.

Der Drehwinkel $\mathrm{d} \mu$ wird direkt aus dem Betrag des Rotationsvektors $\mu$ bestimmt. Die differentielle Größe d $\mu$ wird durch die Winkeldifferenz $\Delta \mu$ ersetzt. Insofern wird über einen kleinen Bewegungsbereich interpoliert. Bei kleineren differentiellen Drehwinkeln $\mathrm{d} \mu$ ist die Gefahr groß, dass der zufällige relative Messfehler größer wird. Bei der Berechnung der differentiellen Schraubsteigung $\tau$ nach der 
Formel $\underline{\tau}=\underline{\mathrm{d}} \bullet \underline{\mu} /|\mu|^{2}$ wird der Fehler quadratisch weitergeführt, weil das Quadrat des Winkels im Nenner steht.

\subsection{Präsentation der relevanten Verschraubungsparameter}

Die Schraubachsparameter wurden aus den Tastermesswerten bestimmt (Kapitel 2.3.2, S. 53). Die eingelesenen Tasterwerte wurden initial über 4 Nachbarwerte gemittelt. Die gewonnenen Daten wurden mit LabView® für den Hin- und Rücklauf eines Messzyklus graphisch wiedergegeben.

Graphische Darstellung von:

1. Die momentanen Schraubachsen $\operatorname{dr}(\alpha)$ mit ihren Durchstoßpunkten $\mathrm{R}_{\mathrm{i}}=\left(\mathrm{x}_{\mathrm{i}}, \mathrm{y}_{\mathrm{i}}, \mathrm{z}_{\mathrm{i}}\right)$ durch die Bandscheibenebene mit der Projektion des Wirbelbogensegments.

2. Der absolute Drehwinkel $\alpha$ in Abhängigkeit des Drehmoments T.

3. Hysterese als Ordinatenabschnitt $\Delta \alpha(\mathrm{T}=0)$.

4. Die momentane Steifigkeit $\tau^{\prime}(\alpha)$ in Abhängigkeit des Drehwinkels.

5. Schraubsteigung $\tau=d s / d \alpha$ als ein Versatz entlang der Schraubachse in Abhängigkeit des Drehwinkels.

6. Die Richtungskoordinaten des normierten Vektors e $(\alpha)$ mit Koordinaten $e_{X}, e_{Y}, e_{Z}$ der momentanen Schraubachse in Abhängigkeit des Drehwinkels.

Um die Messergebnisse repräsentativ und übersichtlich darzustellen, werden nur die Ergebnisse eines kompletten Messzyklus in dem eingeschwungenen Zustand präsentiert. 


\subsection{Ablauf der Messung}

1. Vorbereitung der Messapparatur:

- $\quad$ Fixation des Wirbelsegments

- $\quad$ Aufbringung der Entlastung

- $\quad$ Aufbau und Entlüftung des Umwälzsystems.

2. Befüllung der Wasserbehälter des Umwälzsystems + Ausgleichsbehälter:

- $\quad$ kräftefreie Anordnung der Drehmomentwaage

- $\quad$ Aufbau je nach Drehmomentrichtung.

3. Positionierung der statischen, axialen Vorlast von 100-400N:

- $\quad$ Registrierung der Positionsveränderung des oberen Brustwirbels.

4. Einschwingphase des Messsystems von 30 Minuten.

5. Justierung und Kalibrierung auf die Mittelposition der sechs Messtaster.

6. Zyklische Applikation von Drehmomenten:

- $\quad$ max. Drehmoment von 400-600 Ncm.

7. Berechnung der Schraubachsparameter und ihre graphische Darstellung. 


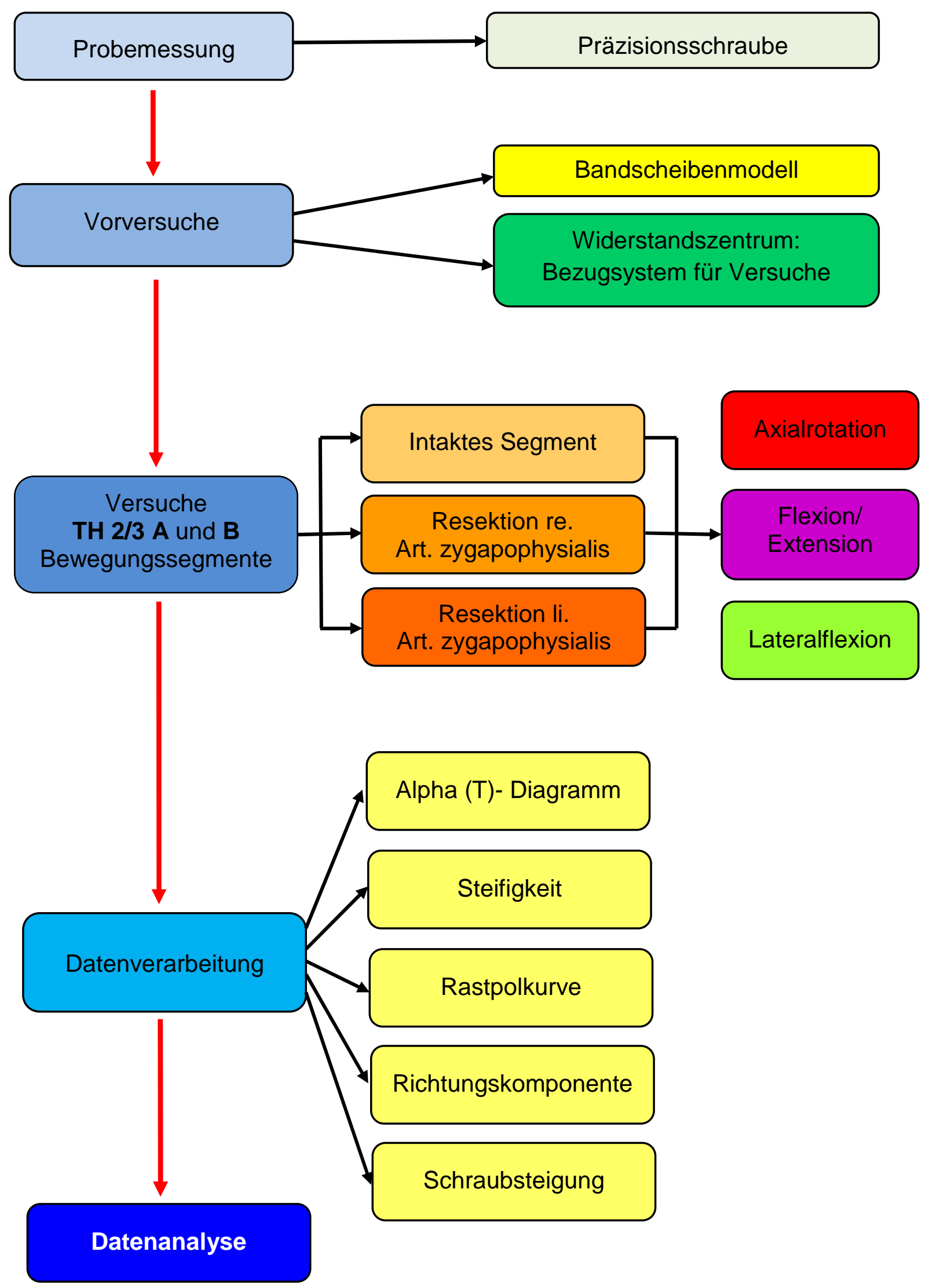

Abb. 2.8.1: Aufbau der Studie. 


\section{Ergebnisse}

Im Folgenden werden die Ergebnisse der Messungen an zwei Th 2/3 Segmenten zusammengefasst. Die beiden Bewegungssegmente (JUNGHANNS 1977) werden im Text Th 2/3 A und Th 2/3 B benannt.

Zuerst wurde die Messapparatur mit Hilfe einer Präzisionsschraube und eines Bandscheibenmodells in Kontrollmessungen überprüft.

Folgend wurden die biomechanischen Eigenschaften des Brustwirbelsegments im Experiment „Axiale Rotation“ untersucht. Das applizierte Drehmoment $\mathrm{T}_{Z}$ hierfür war parallel zur z-Achse ausgerichtet: $T(t)=\left(0,0, T_{Z}(t)\right)$ (Kap. 3.2, S. 78). Für Experiment „Flexions-Extension“ gilt $\mathrm{T}(\mathrm{t})=\left(0, \mathrm{~T}_{\mathrm{Y}}(\mathrm{t}), 0\right)$ (Kap. 3.3, S.117) und für „Lateralflexion" $T(t)=\left(T_{x}(t), 0,0\right)$ (Kap. 3.4, S. 139).

Ein Messzyklus beinhaltete eine zeitliche Veränderung des Drehmoments vom Minimum über das Maximum und wieder zurück zum Minimum. Der beginnende erste Halbzyklus entspricht dabei einer mathematisch-positiven z-Richtung (d.h. eine Linksdrehung) und der folgende zweite Zyklusteil der negativen z-Richtung (eine Rechtsdrehung).

Die axiale Vorlast $\underline{F}_{z}$ wurde in ihrer Größe systematisch verändert und entlang der $x$ - und/ oder $y$-Achse verschoben. Die kompressive Kraft $\underline{F}_{z}$ erzeugte ein Drehmoment $\underline{I}_{x}$ oder $\underline{I}_{y}$. Das Drehmoment führte zu einer Verkippung des oberen Wirbels Th2 gegen den unteren Th3. Es entstanden andere Kontaktpunkte auf den Gelenkflächen der Artt. zygapophysiales und die Gelenkführung änderte sich. In dieser Studie wurde versucht all die Möglichkeiten der Gelenkführung aufzuzeichnen und zu differenzieren. Die sukzessive Resektion der beiden Artt. zygapophysiales an den beiden Th2/3- Segmenten sollte die biomechanische Relevanz der Komponenten demonstrieren.

Um die räumliche Einordnung der Rastpolkurven zu verdeutlichen, wurde auf den Projektionsebenen der entsprechende Schnitt durch den Wirbelkörper und die Artt. zygapophysiales präsentiert. 
Standarddatensätze der Schraubachsparameter

1. Die Rastpolkurve $r_{i}=r(\alpha)$, durch eine gewählte Symmetrieebene mit Durchstoßpunkten $R_{i}=\left(x_{i}, y_{i}, z_{i}\right)$ der momentanen Schraubachse $d_{r}(\alpha)$ :

- $\quad x y$-Ebene mit $R_{i}=\left(x_{i}, y_{i}, 0\right)$ für ein Drehmoment $T_{z}(t)$

- $\quad x z$-Ebene mit $R_{i}=\left(x_{i}, 0, z_{i}\right)$ für ein Drehmoment $T_{Y}(t)$

- $y z$-Ebene $R_{i}=\left(x_{i}, 0, z_{i}\right)$ für ein Drehmoment $T_{X}(t)$.

2. Drehwinkel-Drehmoment Kennlinie $\alpha(T)$.

3. Gesamtsteifigkeit $B_{G}(\alpha)$.

4. Komponenten $e_{X}, e_{Y}, e_{Z}$ des normierten Richtungsvektors $e(\alpha)$.

5. Schraubsteigung $\tau(\alpha)$. 


\section{1 Überprüfung der Messapparatur}

\subsubsection{Feingewindeschraube}

Die Zuverlässigkeit des Messsystems wurde mit Hilfe eines Objekts mit definierten Eigenschaften getestet. Die Feingewindeschraube wurde im Rahmen einer Gesellenprüfung in der Feinmechanikwerkstatt des IV. Physikalischen Instituts angefertigt (Abb. 3.1.1). Das Werkstück mit Präzisionsgewinde (Schraubsteigung 0,005 $\mathrm{mm} / \mathrm{Grad}$ ) und die entsprechende Kontermutter wurden zentrisch in die obere bzw. untere Pfanne eingesetzt. Der Aufbau und die Vorbereitung des Messsystems erfolgten analog zu den Versuchsbedingungen mit eingebetteten Brustwirbeln. Der Ursprung des Koordinatensystems lag im Zentrum der fixierten Gegenmutter. Die Längsachse der Schraube entsprach der z-Achse. Zusammen mit einer axialen Vorlast von 200N wurde ein zyklisches Drehmoment T appliziert und die Messwerte kontinuierlich aufgezeichnet. Eine axiale Rotation der Schraube mit bekannter Schraubsteigung und ortsfester Schraubachse im Mittelpunkt der Schraube wurde erwartet.
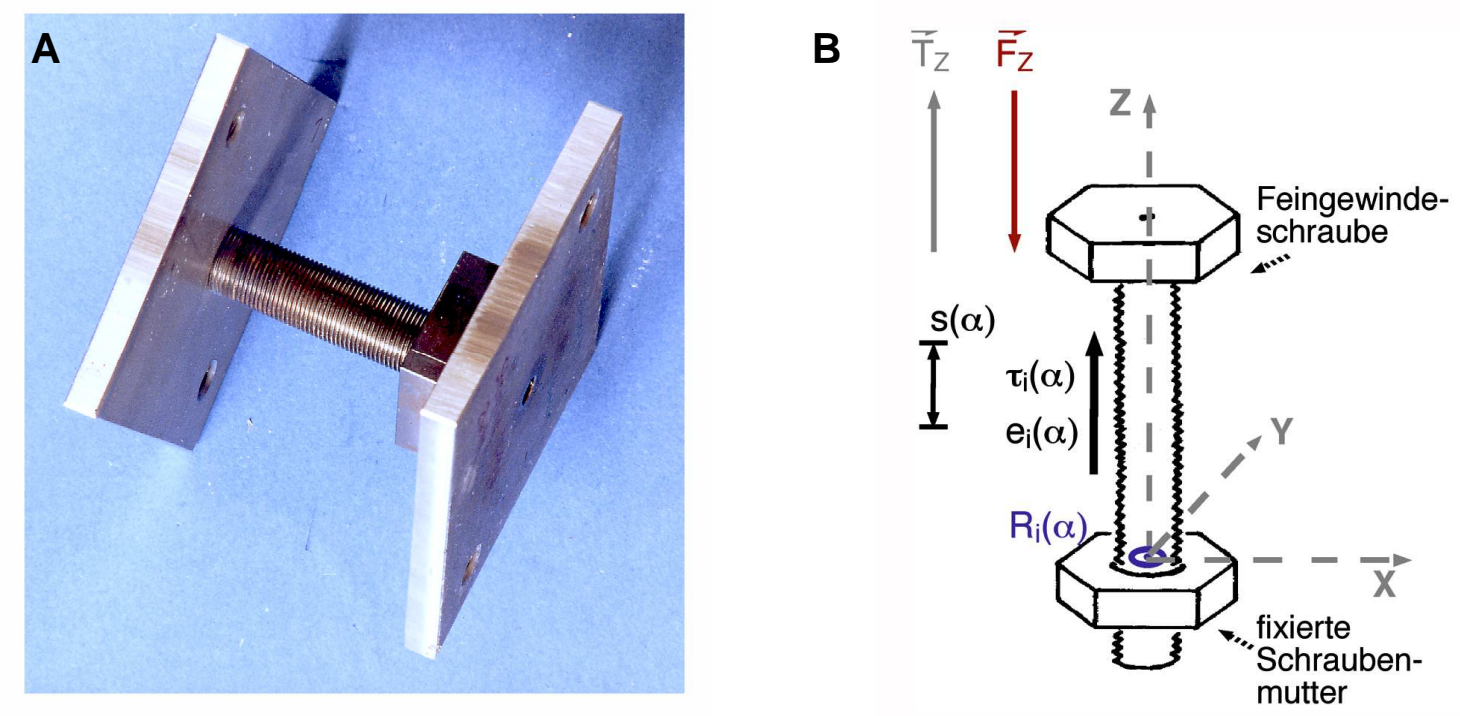

Abb. 3.1.1: Foto der Präzisionsschraube (A) und Schema ihrer Orientierung im Messsystem (B). 
Ergebnisse der Probemessung:

\begin{tabular}{|l|l|l|}
\hline & erwartet & gemessen \\
\hline Drehwinkel & $6,140^{\circ}$ & $6,2^{\circ}$ \\
\hline Schraubsteigung & $0,005 \mathrm{~mm} / \mathrm{Grad}$ & $0,0056 \mathrm{~mm} / \mathrm{Grad}$ \\
\hline Position der Drehachse & zentral & Zentral (Abb. 3.1.2 A.) \\
\hline Richtung der Drehachse & z-Achse & $\mathrm{Z}=1 \quad$ (Abb. 3.1.2 B) \\
\hline Verhalten der Drehachse & ortsfest & ortsfest \\
\hline
\end{tabular}

Die Koordinaten der Durchstoßpunkte der Schraubachsen durch die xy-Ebene (horizontal in der Schraubenmutter) liegen leicht exzentrisch (Abb. 3.1.2 A). Die Pfeile geben die Rotationsrichtung an.

Die Koordinaten des normierten Richtungsvektors $\underline{\mathrm{e}}(\alpha)$ zeigen die Orientierung der Schraubachse im Raum. Ist der Wert einer einzelnen Komponente 1, so findet eine Rotation um die entsprechende Koordinatenachse statt. Abbildung 3.1.2.B zeigt exemplarisch die drei Komponenten ( $e_{X}, e_{Y}, e_{Z}$ ) des Richtungsvektors $e(\alpha)$ in Abhängigkeit des Drehwinkels $\alpha$. Die $e_{z}$-Richtungskomponente ist gleich 1. Somit stehen die momentanen Schraubachsen erwartungsgemäß parallel zur z-Achse des Koordinatensystems.

A

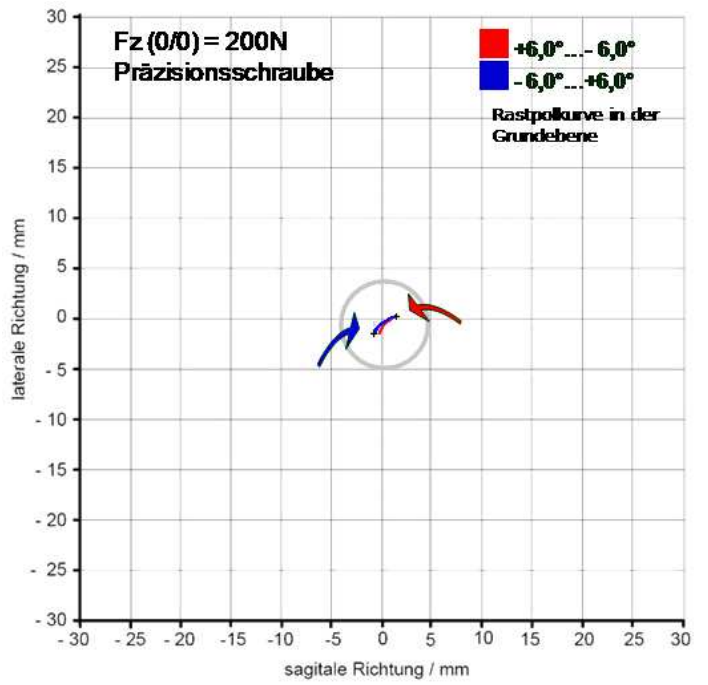

B

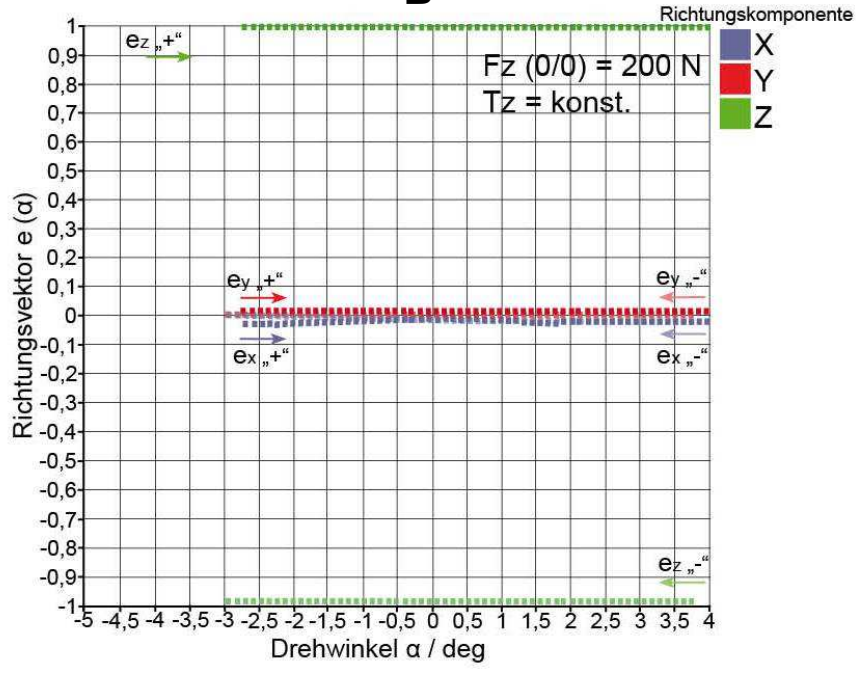

Abb. 3.1.2.A/ B: Auswertung der kinetischen Messungen der Präzisionsschraube.

A) Durchstoßpunkte $R_{i}(\alpha)$ der momentanen Schraubachsen durch eine Referenzebene.

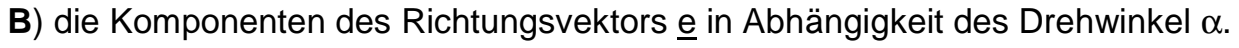


Die aufgezeichnete geringe Abweichung von den Werten der Probemessungen und Erwartungswerten können sowohl durch die Fertigungstoleranz des Messobjekts (wie etwa Spiel im Gewinde), Ungenauigkeit der Einbettung und Positionierung im Messsystem oder der Berechnung selbst liegen. Insgesamt waren die ermittelten Fehler aber vernachlässigbar klein.

\subsubsection{Bandscheibenmodell}

Zwei Kunststoffzylinder (Ø 30mm), mit Tangit verschweißt, sollten die Synarthrose simulieren. Die Höhe der Tangitschicht von 3mm entsprach näherungsweise den elastischen Eigenschaften einer Bandscheibe.

Es wurde zentral eine axiale Vorlast $F_{Z}(0 / 0)=200 \mathrm{~N}$ angebracht und ein linear ansteigendes Drehmoment $T_{z}$ appliziert. Die Durchstoßpunkte der momentanen Schraubachsen durch die xy-Ebene lagen im Zentrum des Zylinders (Abb. 3.1.3 A, S. 75). Das elastische Widerstandszentrum entspricht ungefähr dem Symmetriemittelpunkt.

Ergebnisse der Probemessung:

\begin{tabular}{|l|l|l|}
\hline & erwartet & gemessen \\
\hline $\begin{array}{l}\text { Krehwinkel-Drehmoment } \\
\text { Kennlinie }\end{array}$ & $\begin{array}{l}\text { Hook'sches Verhalten } \\
\text { geringe Hysterese }\end{array}$ & $\begin{array}{l}\text { Hook'sches Verhalten } \\
\text { geringe Hysterese } \\
\text { (Abb. 3.1.3. B) }\end{array}$ \\
\hline Schraubsteigung & $0,0 \mathrm{~mm} /$ Grad & $\begin{array}{l}0,0 \mathrm{~mm} / \mathrm{Grad} \\
\text { (Abb. 3.1.3 D) }\end{array}$ \\
\hline Position der Drehachse & zentral & $\begin{array}{l}\text { zentral, ortsfest } \\
(\text { Abb.3.1.3 A) }\end{array}$ \\
\hline Richtung der Drehachse & $z$-Achse & $\begin{array}{l}z \approx 1 \\
(\text { Abb. 3.1.3. C) }\end{array}$ \\
\hline
\end{tabular}


Die Drehwinkel-Drehmoment-Kennlinie bei maximaler Rotation von $|\alpha| \leq 4,85^{\circ}$ zeigt eine geringe Hysterese, die auf die Viskoelastizität der Tangitscheibe zurückzuführen ist (Abb. 3.1.3 B).

Die $e_{z}$-Richtungskomponente liegt auch hier bei $e_{z}(\alpha)=1$. Die Schraubachsen liegen parallel zueinander und senkrecht zur Horizontalebene des Kunststoffzylinders (Abb. 3.1.3. C)

A

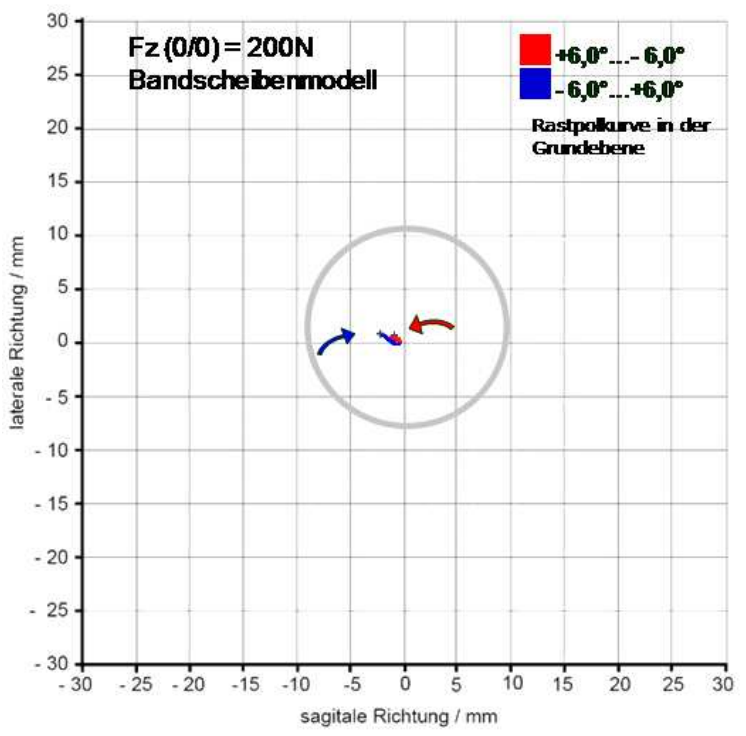

C

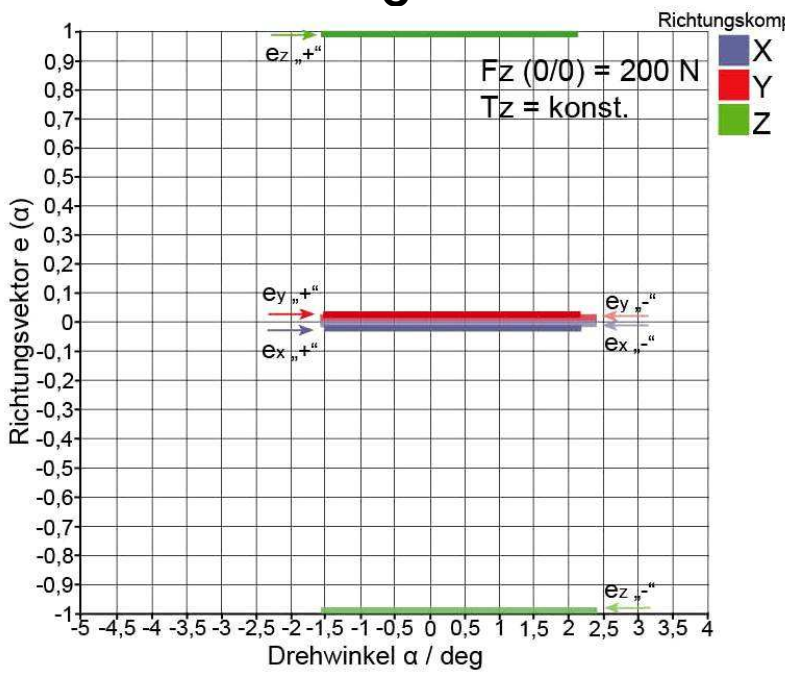

B

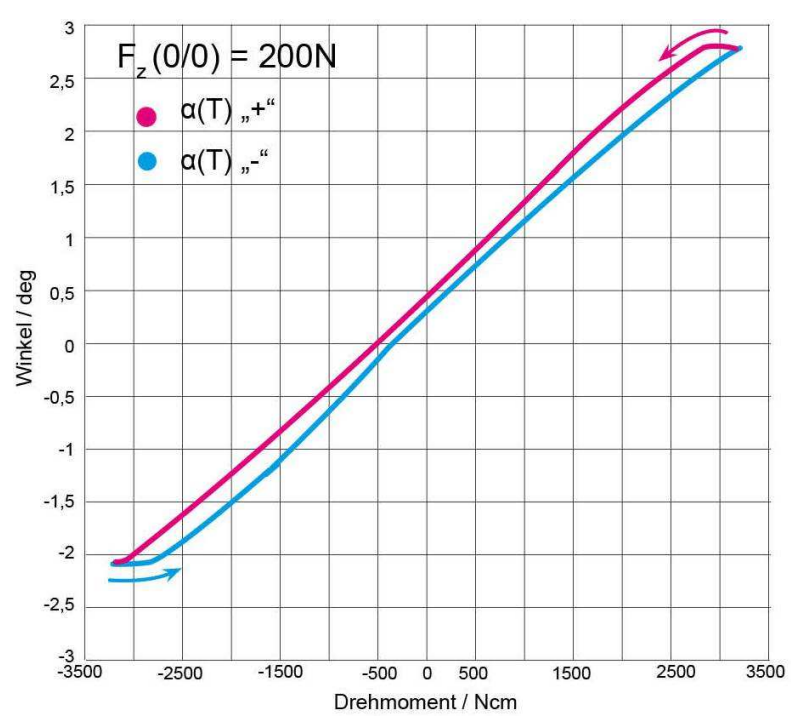

D

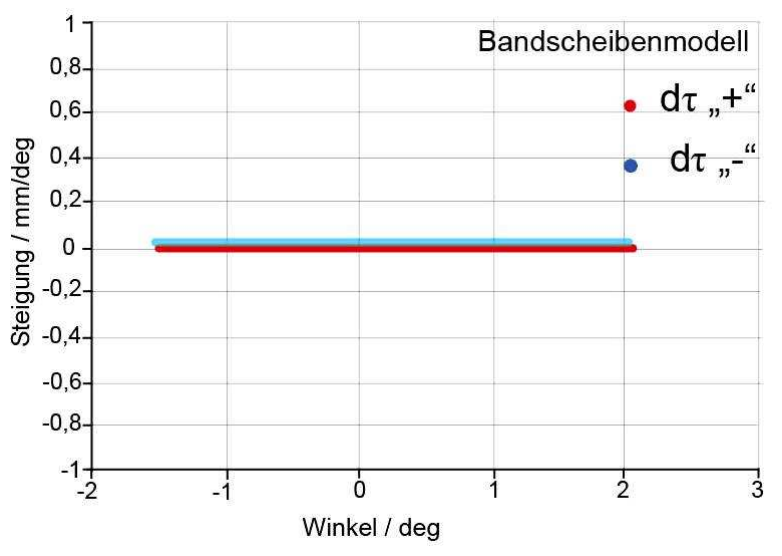

Abb. 3.1.3: Auswertung der kinetischen Messungen des Bandscheibenmodells $\left(T_{z}\right)$.

A) Durchstoßpunkte $R_{i}(\alpha)$ der momentanen Schraubachsen durch eine Referenzebene bei einer Vorlastposition $F_{Z}(0 / 0)=200 N$. B) Drehmoment-Drehwinkel-Kennlinie: Pfeile zeigen die Laufrichtung an. C) die Komponenten des Richtungsvektors $\underline{e}$ in Abhängigkeit des Drehwinkel $\alpha$. D) die momentane Schraubsteigung $\tau(\alpha)$. 


\subsubsection{Auffinden des Widerstandszentrums für axial applizierte Vorlasten}

Das Widerstandszentrum wird als Bezugsystem für die folgenden Experimente unter Applikation von axialen Vorlasten bestimmt. Von diesem experimentell festgelegten „fixen“ Punkt werden dann die Vorlasten in 5mm bzw. 10mm- Schritten in $x$ - und $y$ - Richtung verschoben und die Bewegung des oberen Wirbels aufgezeichnet. Als Widerstandszentrum bezeichnet man den Raumpunkt eines mechanischen Systems, in dem Translation und Rotation entkoppeln (Kapitel 1.4.2.1, S. 29). So führen die Kräfte, die im Widerstandszentrum ansetzen, zu einer reinen Translation und applizierte Drehmomente zu einer reinen Rotation mit einer Achse durch das Widerstandszentrum. Eine Verkippung des Segments bleibt aus.

Das Widerstandszentrum wurde für beide Th2/3- Präparate bestimmt.

Eine Vorlast von $200 \mathrm{~N}$ wurde in sagittale Richtung in $10 \mathrm{~mm}$ - Schritten vom gedachten Widerstandszentrum nach dorsal und ventral verschoben. Durch das Anlegen einer axialen Vorlast verkippt der obere Wirbel unmerklich. Das Segment relaxiert in diese neue gekippte Lage. Um den Relaxationsvorgang zu verstärken, wurde zusätzlich ein zyklisches Drehmoment $T_{Z}$ parallel zur Vorlast appliziert. Somit verkippt das Bewegungssegment in einem vergrößerten Winkel. Das Segment reagiert also empfindlicher auf eine Positionsänderung der Vorlast aus dem Widerstandszentrum heraus.

Die Verkippung des oberen Wirbelkörpers wird durch die Messtaster 1, 2 und 3 (xy-Ebene) gemessen. Die Änderung der momentanen Tasterlänge spiegelt jegliche Verkippung des oberen Wirbels in der Horizontalebene wider. Abbildungen 3.1.4 und 3.1.5 (S. 77) zeigen die Tastermesswerte im Ganzen. Liegt die Vorlast im Widerstandszentrum, so bleibt die Tasterlänge unverändert. Eine Verkippung des Bewegungssegmentes bleibt aus (Abb. 3.1.5).

Das Widerstandszentrum des Bewegungssegmentes Th2/3 A befindet sich bei dem vorliegenden Versuchsaufbau in der Medianlinie am hinteren Rand der Bandscheibe. Beim zweiten Segment Th2/3 B liegt das Widerstandszentrum genau in der Bandscheibenmitte. 


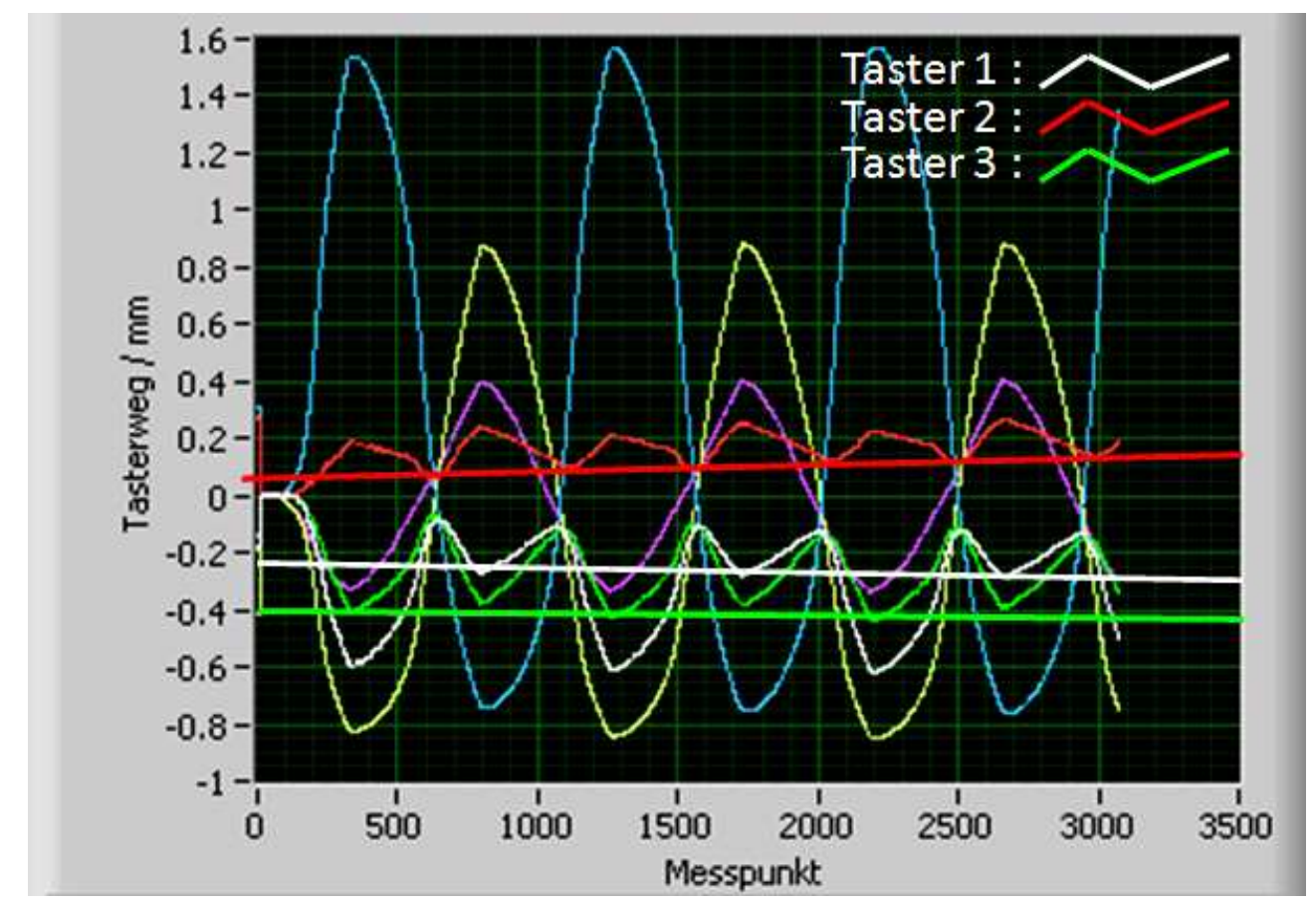

Abb. 3.1.4: Tastermesswerte bei einer Vorlast $200 \mathrm{~N}$ in WZ und Drehmoment $\left(T_{Z}\right)$. Die eingezeichneten Tangenten an den Extremwerten Taster 1, 2 und 3 (xy-Ebene) zeigen eine Verkippung in Richtung Extension. Der ventral positionierte Taster 2 erfährt eine Längenzunahme, dorsal postierten Taster 1 und 3 eine Längenabnahme.

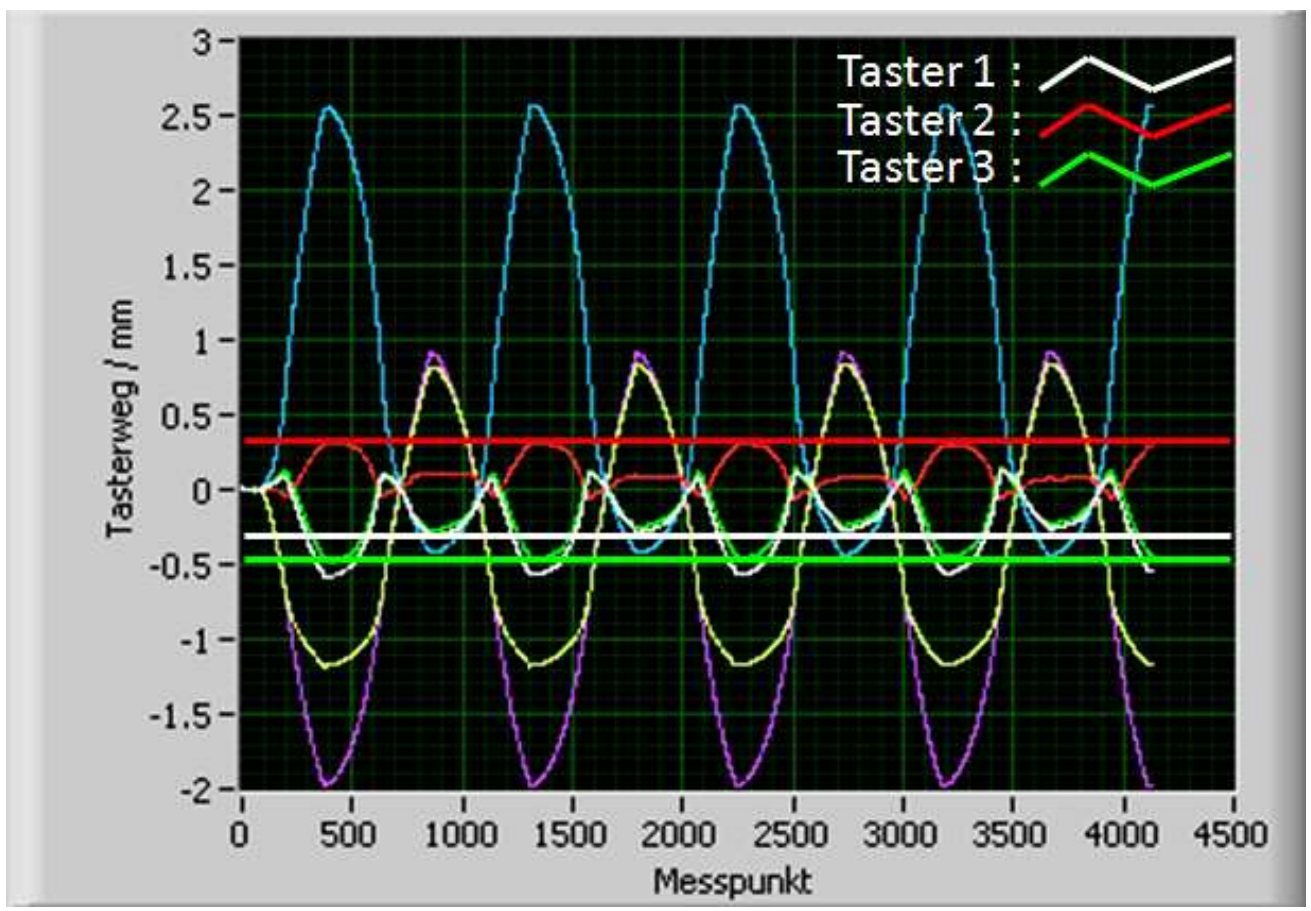

Abb. 3.1.5: Tastermesswerte bei einer Vorlast $200 \mathrm{~N}$ in WZ und Drehmoment $\left(T_{Z}\right)$. Die eingezeichneten Tangenten an den Extremwerten Taster 1, 2 und 3 ( $x y$-Ebene) zeigen einen geraden Verlauf. Der obere Wirbelkörper wird nicht verkippt. 


\subsection{Axiale Rotation $\underline{I}(t)=\left(0,0, I_{z}(t)\right)$}

Im Experiment „Axiale Rotation“ wurde ein zyklisches, sich linear veränderndes Drehmoment $\underline{I}_{z}$ aufgebracht. Die Größe der axialen Vorlast $F_{Z}$ und der Ort inrer Kraftwirkungslinie $\left(\mathrm{x}_{\mathrm{F}}, \mathrm{y}_{\mathrm{F}}\right)$ wurde variiert. Danach wurde die rechte Art. zygapophysialis (Th2/3 A und B) gesamt der Gelenkkapsel entfernt, anschließend das zweite Bogengelenk reseziert. Zwischen diesen Schritten wurden die statischen und dynamischen Messungen wiederholt.

\subsubsection{Bewegungssegment Th2/3 A}

\subsubsection{Rotation ohne axiale Vorlast; $\mathrm{T}(\mathrm{t})=(0,0, \mathrm{Tz}(\mathrm{t})), \mathrm{F}=(0,0,0)$}

\section{Rastpolkurven}

Der geometrische Ort aller

Durchstoßpunkte $R_{i}=\left(x_{i}, y_{i}, 0\right)$ der momentanen Schraubachsen $r_{i}(\alpha)$ mit der Bandscheibenebene erfasst quantitativ die Wanderung der IHA $\left(T_{Z}\right)$.

Ist die $F_{Z}=0$, so stellen sich die Schnittpunkte $R_{i}$ der momentanen Schraubachsen $r_{i}(\alpha)$ durch die Horizontalebene der Bandscheibe des oberen Wirbelkörpers nach ventral auslaufend

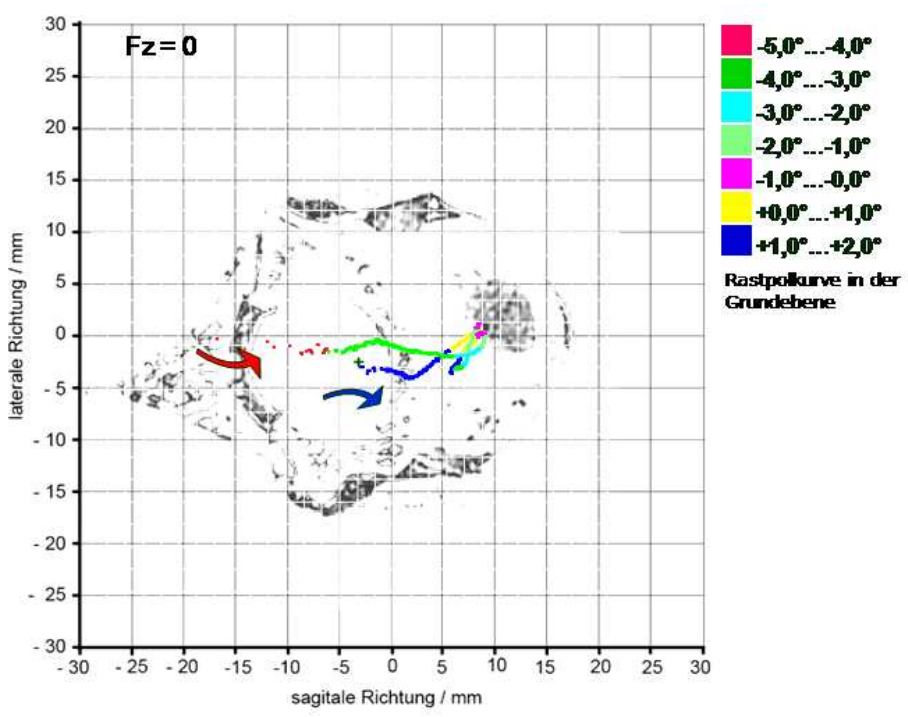
Abb. 3.2.1 (Th2/3 A): Ansicht von kranial. Durchstoßpunkte $R_{i}(\alpha)$ der momentanen Schraubachsen für eine Drehung in positiver und negativer Richtung $\left(\mathbf{F}_{\mathbf{Z}}=\mathbf{0}\right)$. Die gebogenen Pfeile geben die Laufrichtung der Rastpolkurve und die Rotationsrichtung des oberen Wirbels an. bogengelenken dar (Abb.3.2.1).

Die Durchstoßpunkte $R_{i}$ der momentanen Schraubachsen wandern gegenläufig zur Rotationsrichtung des oberen Wirbels - also für eine „,“- Rotation im mathematisch positiven Sinne gegen den Uhrzeigersinn vom rechten zum linken Gelenk. 
Ändert sich die Laufrichtung, springt der Durchstoßpunkt nach dorsal leicht zur gegenüberliegenden Seite versetzt.

Bei dem Segment Th2/3 A (Abb. 3.2.1, S. 78) in Linksdrehung wandert die Schraubachse von dorsal kommend nach ventral. Sie läuft nahezu gerade nach vorn, macht mittig in der Bandscheibe einen Schlenker nach links-dorsal und endet in der Bandscheibenmitte. Bei Bewegungsumkehr springt die momentane Schraubachse nach dorsal. Von dorsal kommend beschreibt die Rastpolkurve ebenfalls einen leicht konvexen Bogen und endet mit einem weiteren Schlenker in der Bandscheibenmitte.

Die Schraubachsen sind also nicht ortsfest und liegen weder im Widerstandszentrum $W_{Z}$ der Bandscheibe, noch dorsal der Wirbelbogengelenke wie in der Literatur oft angenommen (KAPANDJI 1985, KRISMER 1996).

\section{Drehwinkel- Drehmoment- Kennlinie}

Es wird ein zyklisches sich dreieckig änderndes Drehmoment $T(t)$ appliziert. Der Bewegungsumfang (ROM) beträgt $6^{\circ}$ mit maximal angreifendem Drehmoment von $600 \mathrm{~N} / \mathrm{cm}$. Die DrehwinkelDrehmoment-Kennlinie der $\alpha(\mathrm{T})$-Funktion ist deutlich sigmoid geformt (Abb. 3.2.2). Jeder Halbzyklus (Pfeil) beginnt mit einem geringen Anstieg von $\alpha$, es folgt ein mittlerer linearer Teil, danach, trotz weiter linear ansteigendem Drehmoment $\mathrm{T}(\mathrm{t})$, folgt $\alpha$ dem nur noch

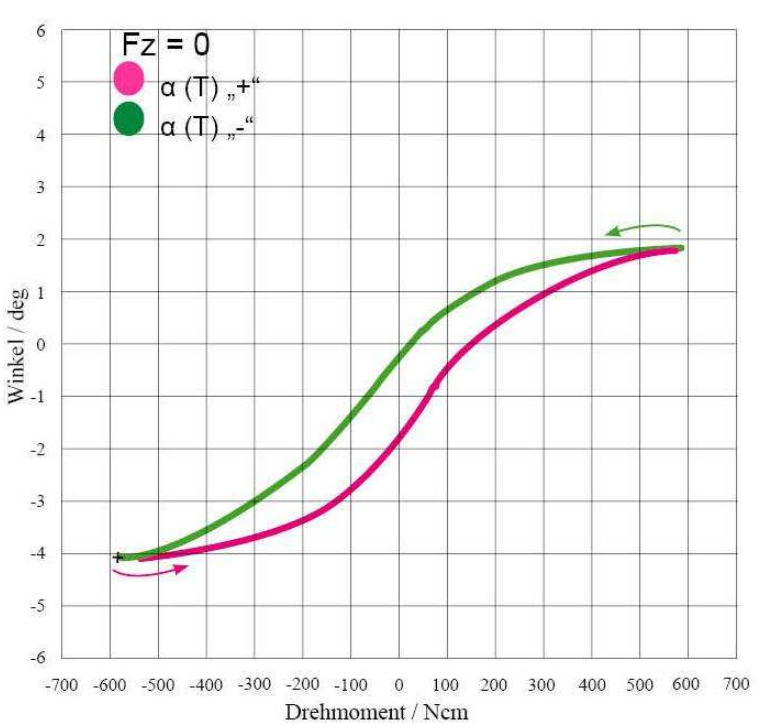

Abb.3.2.2: Drehmoment-Drehwinkel-Kennlinie; die Pfeile zeigen die Laufrichtung an. sublinear. Der Hin- und Rücklauf eines Messzyklus fallen nicht zusammen. Sie zeigen eine messbare Hysterese $\Delta \alpha(T=0)$. 
Der Verlauf der $\alpha(T)$-Funktion ist mit den Last-Deformationskurven von WILKE (1993), PANJABI ET AL. (1981) und MYERS ET AL. (1991) vergleichbar. Der Verlauf einer Last-Deformationskurve wird in einen physiologischen Bewegungsbereich (ROM), in eine neutrale Zone (NZ) mit minimaler Steifigkeit und in eine elastische Zone (EZ) eingeteilt. Durch die anschließend folgende plastische Zone wird das Präparat verformt und dadurch geschädigt.

Die differentielle Steifigkeit des Segments $d T_{z} / d \alpha$ entspricht der reziproken Steigung der $\alpha\left(T_{z}\right)$-Diagramme.

\section{$\underline{\text { Rotationssteifigkeit } B_{G}(\alpha)}$}

Die Gesamtsteifigkeit $B_{G}(\alpha)$ des Segments ohne Applikation einer axialen Vorlast zeigt eine deutlich hohe anfängliche Rotationssteifigkeit ( $>450 \mathrm{Ncm} / \mathrm{deg}$ ) und die Erhöhung am Ende eines Halbzyklus $\left(B_{G} \cong 400\right.$ $\left.\mathrm{Ncm} /{ }^{\circ}\right)$. Für $\alpha=0$ betrug sie $B_{G} \cong 90$ $\mathrm{Ncm} /{ }^{\circ}$ (Abb. 3.2.3) Die minimale Steifigkeit betrug $50 \mathrm{Ncm} /{ }^{\circ}$ für $\alpha=-1^{\circ}$.

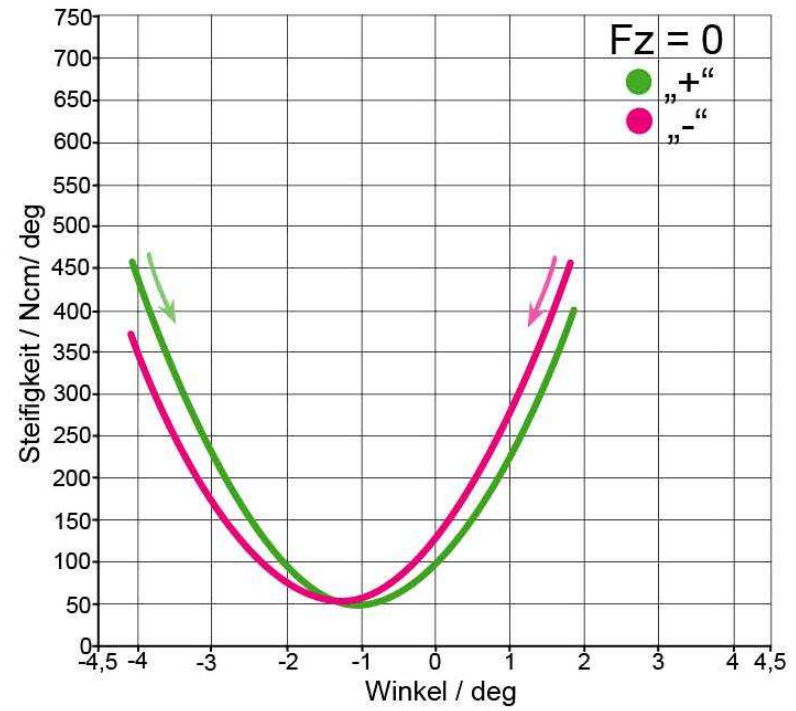

Abb.3.2.3: Die Rotationssteifigkeit $B_{G}(\alpha)$ in Abhängigkeit des Drehwinkels. Der erste Halbzyklus grün (Rechtsdrehung $(,,+)$,$) und zweiter$ Halbzyklus pink (Linksdrehung (,,")).

\section{Richtungsvektor der Schraubachse e ( $\alpha$ )}

In Abbildung 3.2.4 (S. 81) wird das Verhalten der einzelnen Komponenten des Einheitsvektors $\mathrm{e}(\alpha)$ der IHA gezeigt. Die Ausrichtung einzelner Vektoren gibt den Aufschluss über die genaue Position und Neigung der Schraubachse zur bestimmten Ebene. Der Wert der z-Komponente ist $e_{z}(\alpha)=1$ für Rechts- und Linksdrehung. Die anderen Komponenten sind $e_{Y}= \pm 0,25$ und $e_{X}= \pm 0,1$. Nur für Dreh- 
winkel $|\alpha| \leq-1,5^{\circ}$ und $|\alpha| \geq 0,5^{\circ}$ sind alle Einheitsvektoren annähernd parallel zu einander, so dass die resultierenden Schraubachsen nur für kleine Winkelbereiche parallel zum Drehmomentsvektor liegen. Der Richtungsvektor $\mathrm{e}(\alpha)$ steht dann senkrecht zur Bandscheibenebene.

Mit ansteigendem Drehwinkel $\alpha$ verkippt die Schraubachse seitlich. Im Laufe der Linksdrehung ist eine Vergrößerung der $e_{Y}(\alpha)$ zu verzeichnen.

Sie erreicht maximal $0,1^{\circ}$ bei $\mathrm{e}_{\mathrm{z}}(\alpha)$ $=0,99^{\circ}$, was einem Neigungswinkel $\theta_{y}$ der Schraubachse von $5,7^{\circ}$ nach

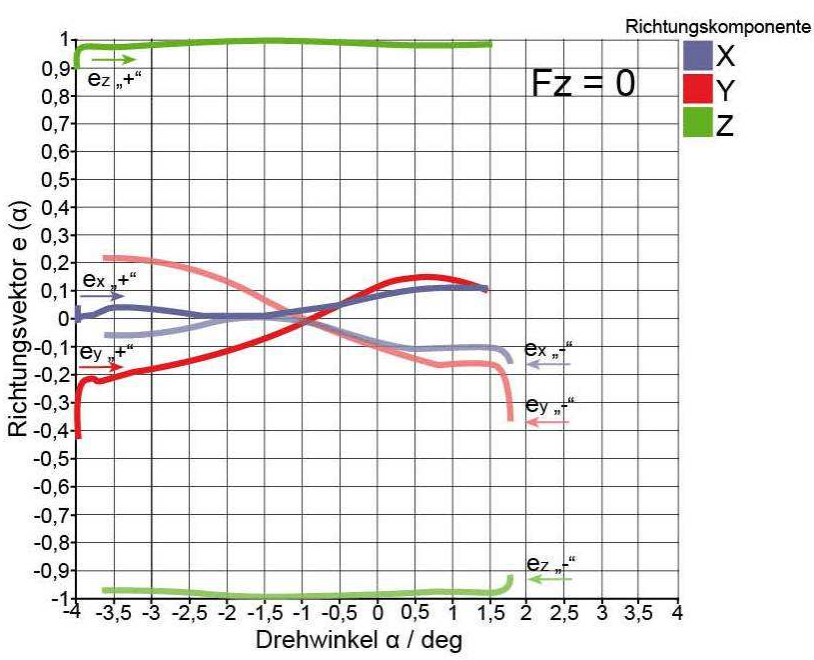

Abb. 3.2.4: Die Komponenten des Richtungsvektors $\underline{e}$ (Th2/3 A) in Abhängigkeit des Drehwinkels $\alpha$ für einen kompletten Messzyklus links lateral entspricht. Eine Verkippung der momentanen Schraubenachse nach rechts bei einer Rechtsdrehung beträgt $11,5^{\circ}$ von ih rer senkrechten Ausrichtung. Somit ist die Verkippung bei der Rechtsdrehung doppelt so stark wie bei der Linksdrehung.

Die $e_{x}$-Komponente gibt Aufschluss über das Verhalten der momentanen Schraubachse in der Horizontalebene. So ist der Verkippungswinkel $\theta_{x}$ maximal $(\alpha)=5,7^{\circ}$ für eine Linksdrehung. Bei maximaler Rechtsdrehung $e_{x}=-0,07^{\circ}$ entspricht die Verkippung der Schraubachse dem Neigungswinkel von $4,08^{\circ}$ zur Horizontalebene.

Damit verkippt die momentane Schraubachse bei der Linksdrehung um 5,7 nach links-lateral und um den gleichen Betrag nach dorsal und bei der Rechtsdrehung um $11,5^{\circ}$ nach rechts-lateral und um $4,08^{\circ}$ nach dors al. 


\section{Schraubsteigung $\tau(\alpha)$}

Zusätzlich zur Rotationsbewegung kommt es zu einem Versatz ds entlang der differentiellen Schraubachse $\operatorname{dr}(\alpha)$ oder zur einen Translation. Die differentielle Schraubsteigung $\tau(\alpha)$ wird in der Einheit $\mathrm{mm} / \mathrm{grad}$ aufgetragen und über den Drehwinkel $\alpha$ präsentiert (Abb. 3.2.5). Die Bewegung ist für Hinund Rücklauf unterschiedlich.

Steigt für die Rechtsdrehung die negative Schraubsteigung $\tau(\alpha)$ mit zuneh-

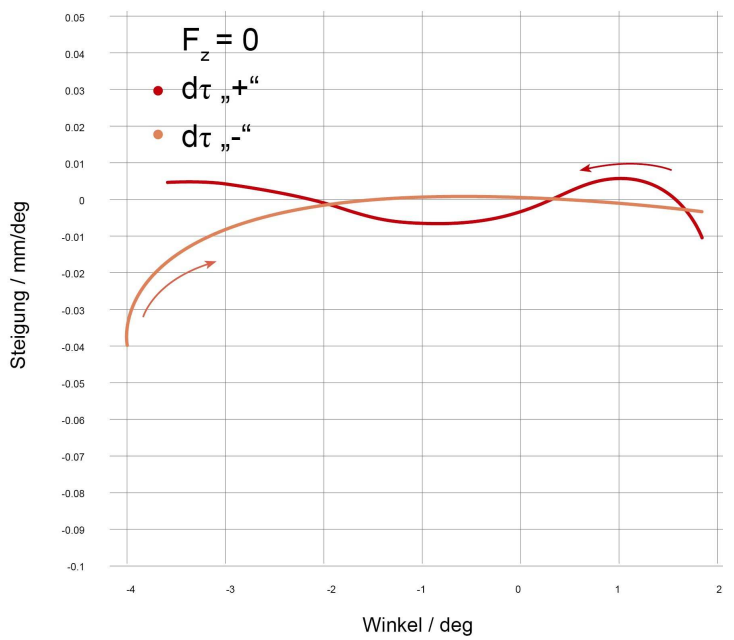

Abb. 3.2.5: Die momentane Schraubsteigung $\tau(\alpha)$ in Abhängigkeit des Drehwinkels $\alpha$. mendem Winkel $\alpha$ an, so entfernen sich die Wirbelkörper voneinander und die Bandscheibe wird mit $s_{\max }=0,035 \mathrm{~mm}$ leicht elongiert. Für die Linksdrehung ist die Bewegung uneben. Die Schraubsteigung steigt zunächst von $-0,01 \mathrm{~mm} /{ }^{\circ}$ bis etwa $0,005 \mathrm{~mm} /{ }^{\circ} \mathrm{im}$ Winkelbereich $\alpha=1,8^{\circ}-1^{\circ}$, dann fällt sie zurück ins Negative bis $\alpha=-1^{\circ}$, danach folgt kontinuierlich ansteigend die positive Schraubsteigung $\tau(\alpha)$ bis etwa $0,005 \mathrm{~mm} /{ }^{\circ}$. Die Bandscheibe wird $\mathrm{mit} \mathrm{s}_{\max }=0,015 \mathrm{~mm}$ ebenfalls leicht elongiert.

\subsubsection{Erhöhung des Betrags der axialen Vorlast}

$$
\underline{I}(t)=\left(0,0, \underline{I}_{z}(t)\right), F=\left(0,0, F_{z}\right)
$$

\section{Rastpolkurven}

Im ermittelten Widerstandszentrum $W_{Y}$ wurde nun die axiale Vorlast $F_{Z}$ schrittweise um $100 \mathrm{~N}$ von anfangs $100 \mathrm{~N}$ auf $400 \mathrm{~N}$ erhöht und ein zyklisches Drehmoment $T_{z}(t)$ appliziert. Es resultiert eine Kraftschraube (vgl. Kap 1.2). Die Erhöhung der Vorlast $F_{Z}$ verstärkt die Kompression der Bandscheibe und verändert den Kraftschluss der Gelenke. Bei der Verkippung des oberen Wirbels im Winkelbereich $\alpha$ $=0 \pm 2^{\circ}$ befinden sich die Rastpolkurven annähernd am gleichen Ort (Abb. 3.2.6). 
Beim Bewegungssegment $\mathbf{A}$ verschiebt sich die Rastpolkurve leicht nach ventral und beult sich stärker aus. Eine Erhöhung der Vorlast von $100 \mathrm{~N}$ auf $400 \mathrm{~N}$ veränderte $r(\alpha)$ nicht nennenswert.

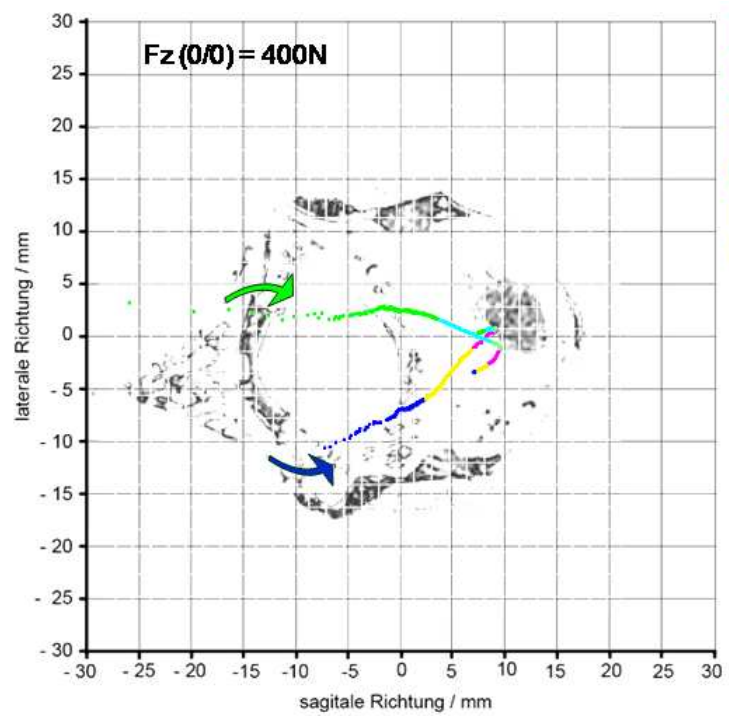

Abb. 3.2.6: (Th2/3 A) Ansicht von kranial. Rastpolkurve $R_{i}(\alpha), F_{Z}=400 \mathrm{~N}$ im WZ.

Drehwinkel- Drehmoment- Kennlinie Das $\alpha(T)$-Diagramm verändert sich unmerklich in Abhängigkeit von der Größe der Vorlast. Für $F_{Z}=400 \mathrm{~N}$ ist die Hysterese $(T=0)$ größer. Der Bewegungsumfang von $6^{\circ}$ bleibt annähernd gleich (Abb. 3.2.7).

Die Vorlastgröße beeinflusst nicht den maximalen Rotationsumfang. Die Tatsache aber, dass der Hystereseumfang ansteigt, kann ein Hinweis darauf sein, dass die inne-

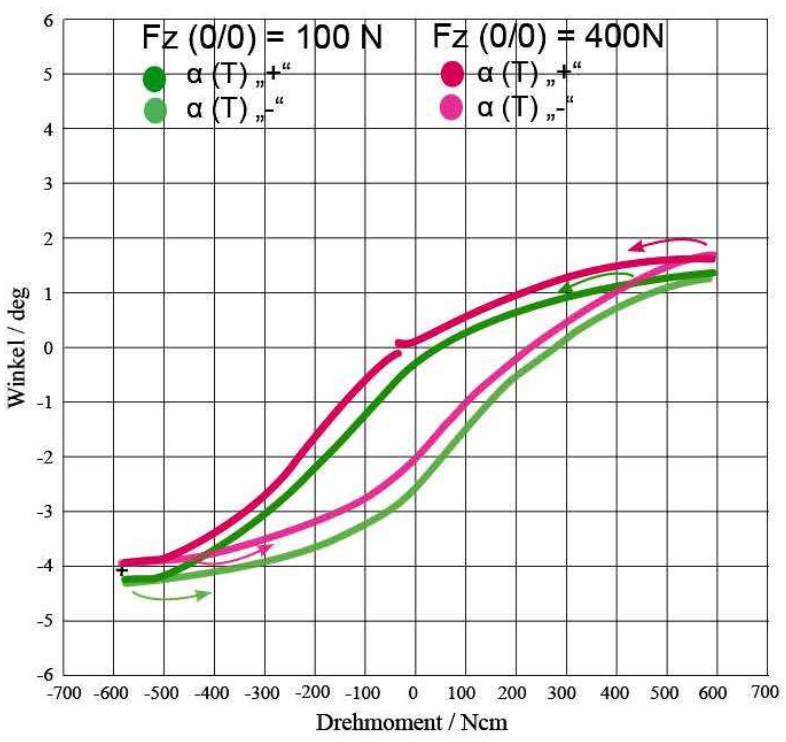
Abb. 3.2.7: Drehmoment-Drehwinkel-Kennlinien. Axialrotation: $F_{Z}=100 \mathrm{~N}, F_{Z}=400 \mathrm{~N}$ re Reibung in der Bandscheibe mit zunehmendem axialem Kraftangriff ansteigt. 


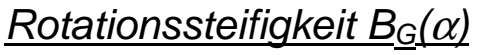

Die Applikation von hohen axialen Vorlasten erzeugt eine hohe anfängliche Rotationssteifigkeit des Segments (750 $\mathrm{Ncm} / \mathrm{deg}$ ) und die Erhöhung am Ende eines Halbzyklus $\left(B_{G} \cong 550 \mathrm{Ncm} /{ }^{\circ}\right)$. Für $\alpha=0$ betrug sie $B_{G} \cong 150 \mathrm{Ncm} /{ }^{\circ}$. Die minimale Steifigkeit verdoppelte sich und betrug $100 \mathrm{Ncm} /{ }^{\circ}$ für $\alpha=-1^{\circ}$ (Abb. 3.2.8).

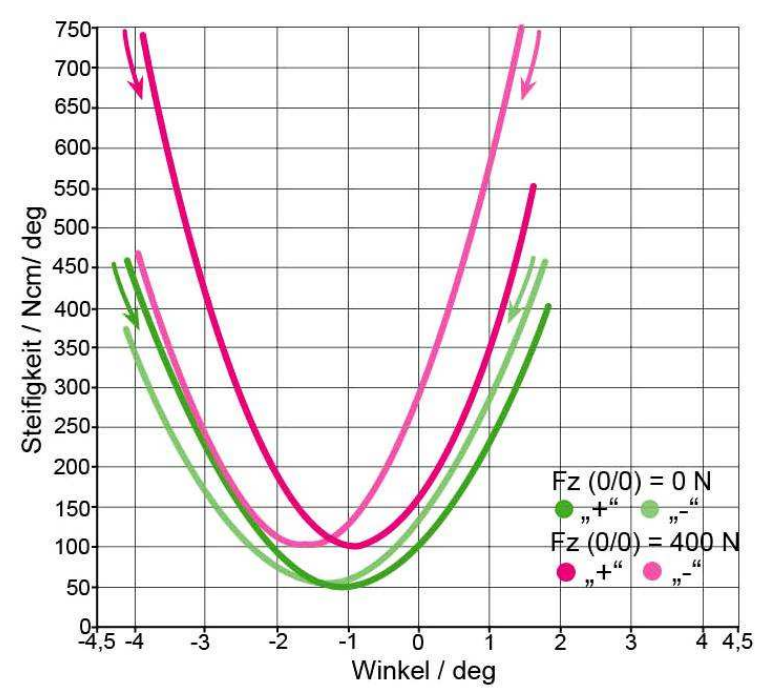

Abb.3.2.8: Die Rotationssteifigkeit $B_{G}(\alpha)$ in Abhängigkeit des Drehwinkels.

Axialrotation in WZ: grün ohne Vorlast, pink mit $400 \mathrm{~N}$ Vorlast.

Für jede Vorlastvariation wurden sowohl die Richtungskomponente der IHA wie auch die momentane Schraubsteigung bestimmt. Qualitative Veränderungen waren gering. Da es keine nennenswerten Unterschiede im Verlauf der jeweiligen Schraubsteigung $\tau=\tau(\alpha)$ und des Richtungsvektors $\underline{\mathrm{e}}(\alpha)$ zu geben scheint, soll eine axiale Vorlast $F_{Z}=200 \mathrm{~N}$ ausreichen, um den maximalen Effekt $z u$ beschreiben. 


\subsubsection{Verschiebung entlang der $x$-Achse}

$$
I F_{Z} I=200 N ; \underline{I}(t)=\left(0,0, \underline{Z}_{Z}(t)\right), F\left(x_{F} / 0\right)=\left(0,0, F_{Z}\right)
$$

In der Abbildung 3.2.9 wird die Verschiebung der applizierten Vorlast $F_{Z}\left(x_{F} / 0\right)=$ $200 \mathrm{~N}$ schematisch dargestellt. Die Vorlast wurde systematisch in $5 \mathrm{~mm}$-Schritten entlang der x-Achse verschoben. Es kommt zu einer Flexion (Verkippung des oberen Wirbelkörpers nach ventral) und einer Extension (Verkippung des oberen Wirbels nach dorsal).

Das Widerstandszentrum $\mathrm{W}_{\mathrm{Y}}$ für Flexion/ Extension bei statischer Kraftapplikation liegt annähernd in der Position $x_{F}=-5 \mathrm{~mm}$ für Th2/3 $A$ und $x_{F}=+5 \mathrm{~mm}$ für Th2/3 B.
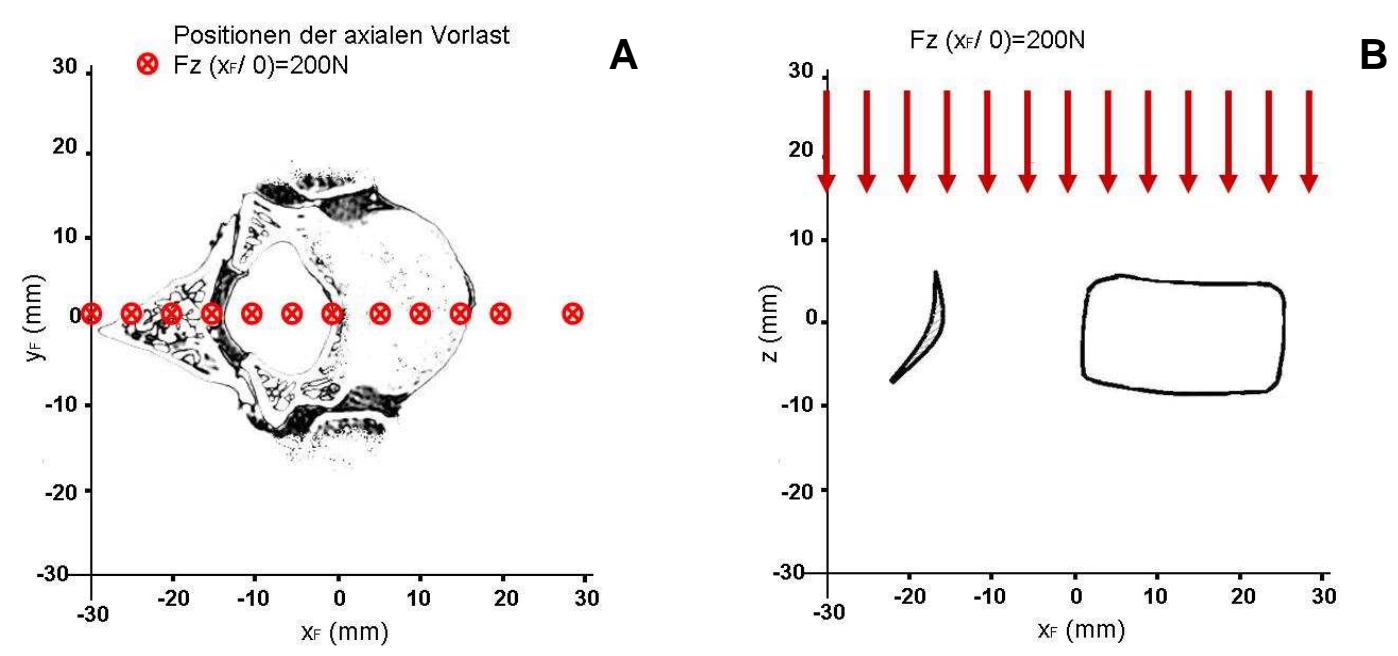

Abb. 3.2.9: Auswertung der statischen Messungen für beide Bewegungssegmente (Th2/3 A und B):

A) Durchstoßpunkte der Kraftwirkungslinie von $\mathrm{F}_{Z}\left(\mathrm{x}_{\mathrm{F}} / 0\right)$ durch die $\mathrm{xy}$-Referenzebene von kranial betrachtet.

B) Position der Kraftwirkungslinie $F_{Z}\left(x_{F} / 0\right)$ in der Medianebene von rechts betrachtet. 


\section{Rastpolkurven $r(\alpha)$}

Eine konstante Vorlast $F_{Z}=200 \mathrm{~N}$ wurde in 5 -mm-Schritten entlang der $x$-Achse verschoben (Abb. 3.2.9) und ein zyklisches Drehmoment $T_{z}(t)$ angebracht. Die Rastpolkurven weisen deutliche Unterschiede in Abhängigkeit von der Vorlastposition $X_{F}$ auf. Vergleichend sollte zuerst das Verhalten von IHA für eine im Widerstandszentrum positionierte Vorlast beschrieben werden.

\section{zentrale Vorlast}

Im ermittelten Widerstandszentrum $W_{Y}$ wurde nun die axiale Vorlast $F_{Z}$ von $200 \mathrm{~N}$ aufgebracht und ein zyklisches Drehmoment $T_{z}(t)$ appliziert. Es resultiert eine Kraftschraube (vgl. Kap 1.2). Die axiale Vorlast $F_{Z}$ bewirkte eine Kompression der Bandscheibe. Beide Gelenke sind kraftschlüssig. Der Verlauf der Rastpolkurve ist der in Abbildung 3.2.6 gezeigten gleich.

\section{dorsale Vorlast}

Es wird eine Vorlast $F_{Z}=200 \mathrm{~N}$ dorsal des ermittelten Widerstandszentrums appliziert $\left(\mathrm{x}_{\mathrm{F}}<0\right)$. In Linksdrehung wandert die Schraubachse von dorsal kommend nach ventral beginnend mit einer Verkippung ( $\alpha=-59$ (Abb. 3.2.10). Sie läuft hier quer zur Mitte der Bandscheibe und leicht zur Gegenseite versetzt, macht mittig in der Bandscheibe einen weiten Bogen nach links-dorsal und endet dort. Bei der Richtungsumkehr springt die

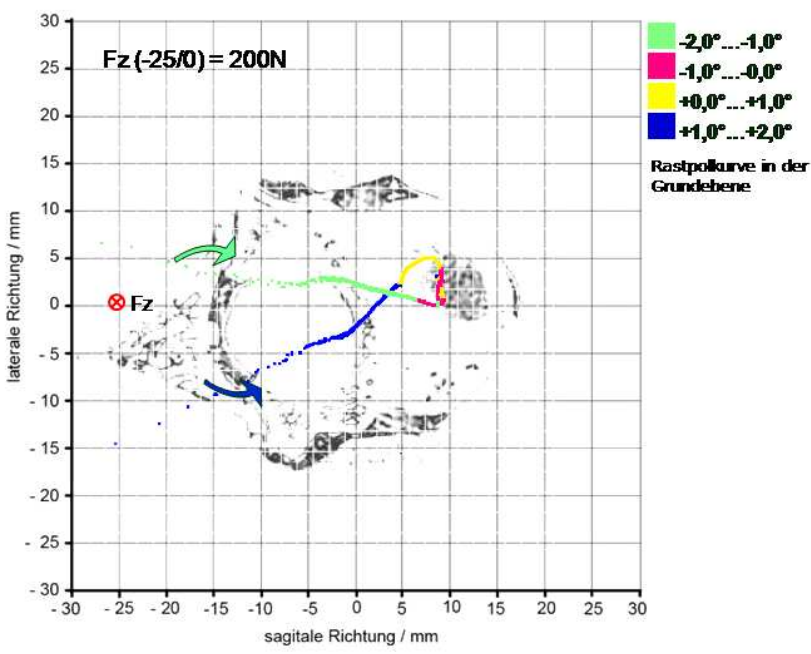

Abb. 3.2.10: Rastpolkurven von Th2/3 A Durchstoßpunkte $\mathrm{R}_{\mathrm{i}}(\alpha)$ der momentanen Schraubachsen bei dorsaler Vorlastposition $(\mathbf{X} \mathbf{i}=-25 \mathrm{~mm})$ momentane Schraubachse nach dorsal zur Gegenseite nah der rechten Art. zygapophysalis, die Rechtsdrehung setzt ein. In Laufe der Rechtsdrehung beschreibt die Rastpolkurve einen Bogen, der der Linksdrehung nahezu spiegelsymmetrisch ist. 


\section{ventrale Vorlast}

Bei der Positionierung von $\mathrm{F}_{Z}$ ventral des Wirbelkanals ( $\mathrm{X}_{\mathrm{F}}>0 \mathrm{~mm}$ ) beschreibt die Rastpolkurve sowohl für Links-, als auch für Rechtsdrehung einen weit nach dorsal offenen ventral konvexen Bogen. Bei maximaler Rotation verbleiben die Schraubachsen in den lateralen Anteilen der Zwischenwirbelscheibe.

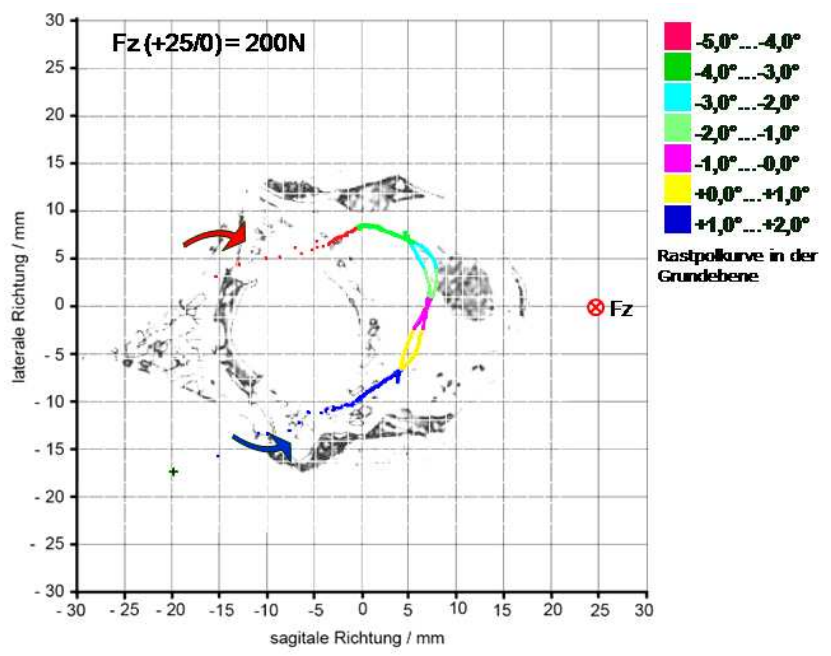

Abb. 3.2.11: Rastpolkurven von Th2/3 A Durchstoßpunkte $\mathrm{R}_{\mathrm{i}}(\alpha)$ der momentanen Schraubachsen bei ventraler Vorlastposition $(\mathbf{X} \mathbf{i}=+25 \mathrm{~mm})$

Die Rastpolkurven von $\mathrm{F}_{\mathrm{Z}}(0 / 0)$ (Abb. 3.2.6, S. 83) im Widerstandszentrum unterscheiden sich qualitativ in der Form im Vergleich zu der vorher betrachteten (Abb. 3.2.11). Bei ventraler Vorlast wandern die Durchstoßpunkte zwischen den beiden Gelenken, wobei die Endpunkte der Rastpolkurve nahe dem Bandscheibenrand liegen.

Wie schon oben erwähnt wurden differentielle Schraubenachsen der jeweiligen absoluten Drehwinkel $\alpha$ mit einem Intervall von $\Delta \alpha=1^{\circ}$ zugeordnet und farbig dargestellt (Abb. 3.2.10 und Abb. 3.2.11).

Der absolute Drehwinkel $\alpha$ ist für $\mathrm{X}_{\mathrm{F}}<0$ (Extension, Abb. 3.2.10) gleichmäßig von $-2^{\circ}$ über $0^{\circ}$ bis $+2^{\circ}$ entlang der Rastpolkurve $r(\alpha)$ verteilt. Das sollte einer konstanten Wanderungsgeschwindigkeit der IHA entsprechen. Die Verteilung des absoluten Drehwinkels $\alpha$ entlang der Rastpolkurve $\alpha$ für $x_{F}>0$ (Flexion, Abb. 3.2.11) zeigt, dass die Rechtsdrehung von $\alpha=-4^{\circ}$ in der Nähe des linken Wirbelgelenkes erfolgt. 
Sukzessive Resektion der Articulationes zygapophysiales Th2/Th3 A

Um das Führungsverhalten der einzelnen Wirbelbogengelenke zu beurteilen, wurde erst die einseitige Resektion des rechten Bogengelenks von Th2/3 A und danach des linken Bogengelenks vorgenommen.

\section{zentrale Vorlast}

Nach der Resektion rechts folgte die Rastpolkurve nur von $x^{\circ}$ bis $y^{\circ}$ der Rastpolkurven des intakten Segments (Abb. 3.2.12 A Vergleich Abb. 3.2.1). Also bleiben die momentanen Schraubachsen $\operatorname{dr}(\alpha)$ bei maximaler Auslenkung - $\alpha$ (Farbe: grün) dorsal der Zwischenwirbelscheibe mittig im Canalis vertebrales und wandern für eine Rotationsbewegung in positiver Richtung (gegen den Uhrzeigersinn in Aufsicht) des oberen Wirbelkörpers in die Bandscheibe, enden mit einem Schlenker nach dorsal und verbleiben dort auch für $\alpha>0$. Die Linksdrehung (gegen den Uhrzeigersinn) entspricht der gleichnamigen Drehung des intakten Segments.

Bei negativer Rotationsrichtung (eine Drehung nach rechts, in Uhrzeigersinn) springt die Rastpolkurve an den dorsalen Bandscheibenrand und verläuft nach ventral. Sie endet mit einem harmonischen Bogen in die Mitte der Bandscheibe.

Nach der beidseitigen Resektion fallen alle Rastpolkurven für $-10<\mathrm{x}_{\mathrm{F}}<+30$ zusammen. Es resultiert eine zentral in der Bandscheibe gelegene Rastpolkurve mit einer geringen Wanderung in $x$-Richtung von $\Delta r_{x}=10 \mathrm{~mm}$. In $y$-Richtung ist die Positionsveränderung $\Delta r_{Y}<5 \mathrm{~mm}$ minimal (Abb. 3.2.12 B, S. 89). Die Schraubachsen sind praktisch ortsfest. 

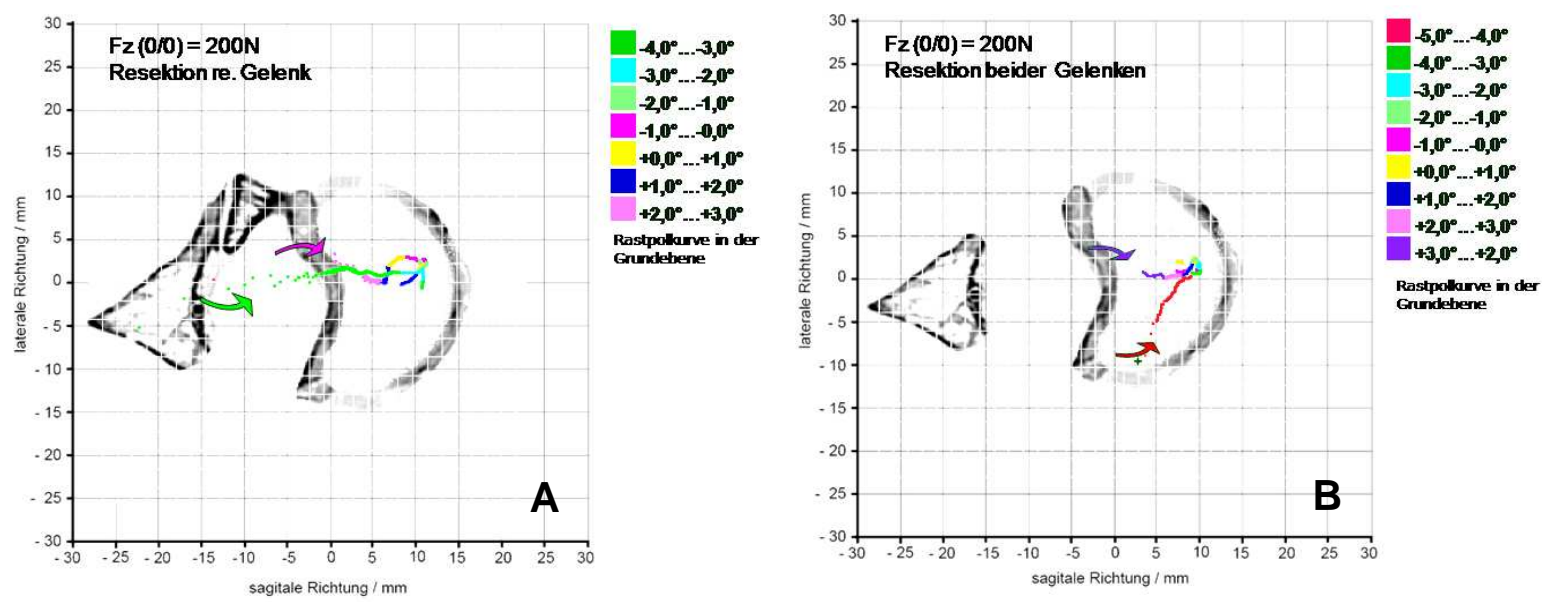

Abb. 3.2.12 A/B: Rastpolkurven von Th2/3 A nach Resektion des rechten Bogengelenks $(\mathbf{A})$ und nach Resektion beider Bogengelenke (B). Die gebogenen Pfeile geben die Laufrichtung der IHA und Rotationsrichtung des oberen Wirbels an.

Die Abbildung 3.2.13 zeigt die vorgenommenen Resektionen an den beiden eingebetteten Segmenten Th 2/3 A und B.
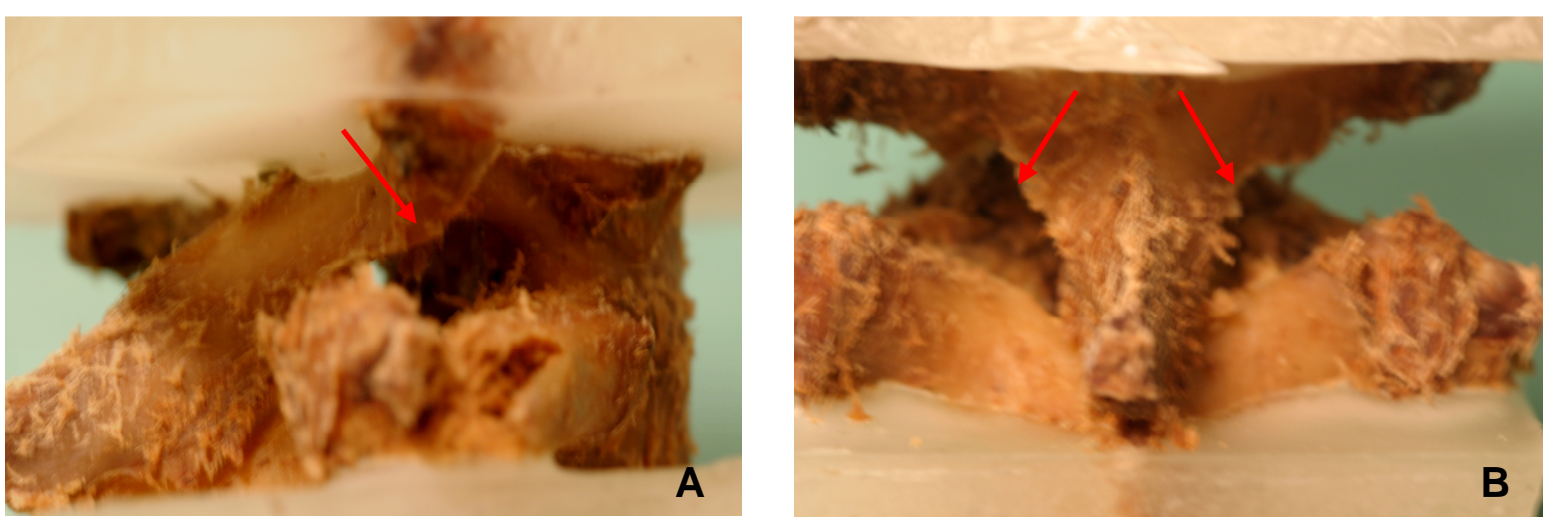

Abb. 3.2.13 : Das Bewegungssegment nach Resektion des rechten Bogengelenks $(\mathbf{A})$ und nach Resektion beider Bogengelenke (B).

Der qualitative Unterschied in der Bewegungsstruktur zwischen ventraler (Abb. 3.2.14 A/B) und weit dorsaler (Abb. 3.2.15 A/B) Vorlastpositionierung ist auch bei der zweiten Resektion vorhanden.

\section{ventrale Vorlast}

Nach Resektion von den rechten Art. zygapophysialis verlagert sich die Rastpolkurve nach ventral in die Bandscheibe (Abb. 3.2.14 A, S. 90). Bei der Linksdrehung wandert die momentane Schraubachse innerhalb der Bandscheibe von links-lateral zum ventralen Bandscheibenrand, so dass sie in der maximalen 
Linksdrehung mittig stehen bleibt. Bei der Rechtsdrehung kommt es zu einem Kraftschluss der Gelenkflächen des linken Bogengelenks. Die Rastpolkurve wandert bei Richtungsumkehr von dorsal kommend in die Bandscheibe, wo sie bei der maximalen Rechtsdrehung rechts lateral endet.

Nach der beidseitigen Resektion liegt die komplette Schraubachsenschar ventral in der Bandscheibe (Abb. 3.2.14 B). Die momentanen Schraubachsen sind, sowohl für Links-, als auch für Rechtsdrehung ventral und mittig in der Zwischenwirbelscheibe lokalisiert.
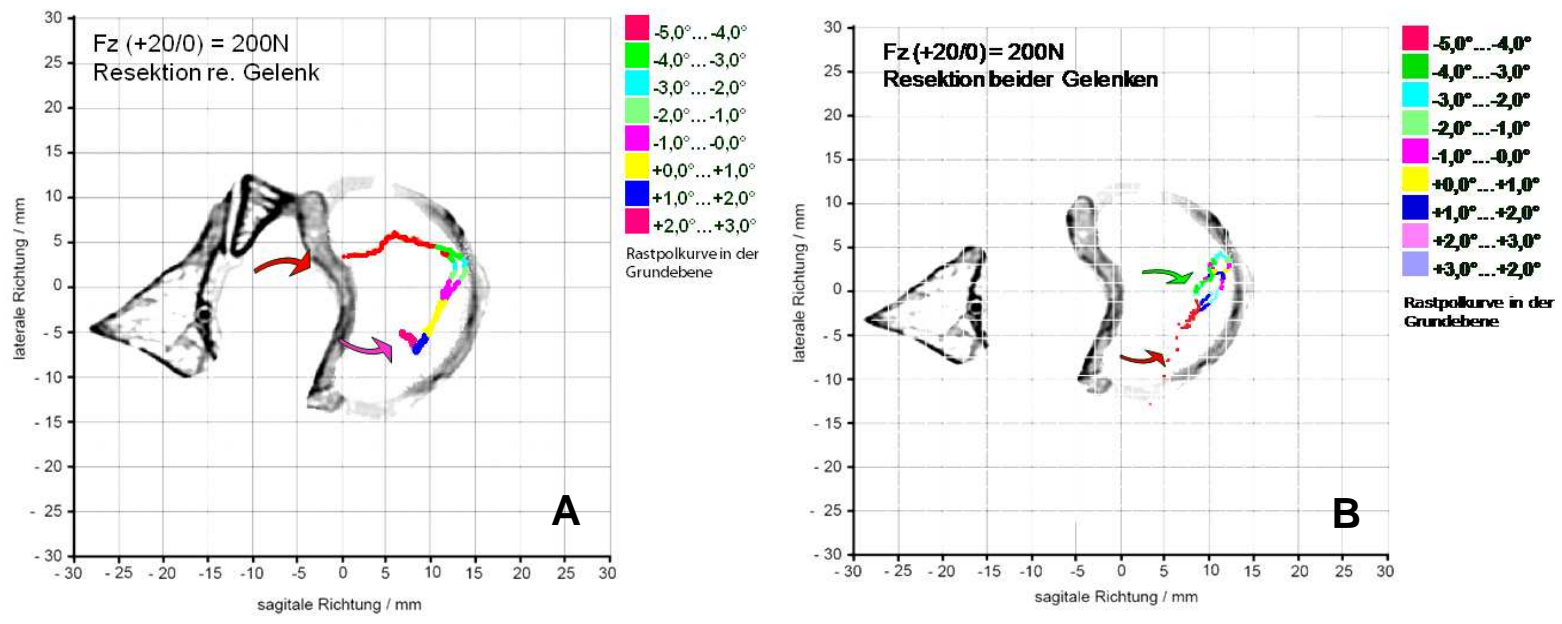

Abb. 3.2.14 A/B: Rastpolkurven von Th2/3 A; Durchstoßpunkte $R_{i}(\alpha)$ der momentanen Schraubachsen bei Vorlastposition ventral: nach Resektion des rechten Gelenks $(\mathbf{A})$ und nach Resektion beider Bogengelenke (B). Die gebogenen Pfeile geben die Laufrichtung der Rastpolkurve und die Rotationsrichtung des oberen Wirbels an.

\section{dorsale Vorlast}

Einen eindrucksvollen Effekt zeigt das Verhalten der momentanen Schraubachsen nach der ersten Resektion für dorsale Vorlasten (Abb. 3.2.15 A, S. 91). Die Rastpolkurven verlagern sich zu der Seite des vorhandenen Bogengelenks. Im Laufe der Rechtsdrehung wandert die momentane Schraubachse von dorsal mitten durch den Canalis vertebralis in die Bandscheibe, macht dort einen weiten Schlenker nach rechts-lateral und endet in der ventralen Bandscheibenmitte.

Nach der zweiten Resektion verlaufen die Rastpolkurven für Hin- und Rücklauf mittig und enden im ventralen Bereich der Bandscheibe (Abb. 3.2.15 B, S. 91). Vergleicht man die Rastpolkurven des Bewegungssegments nach der beidseitigen Resektion bei ventralen und dorsalen Vorlasten, so fällt der unterschiedliche 
Verlauf der IHAs auf. Bei den dorsalen Vorlasten wandert die Rastpolkurve für Linksdrehung, wie auch für die Rechtsdrehung, von dorsal in die Zwischenwirbelscheibe ein. Beginnend mitten in dem Canalis vertebralis, läuft sie geradeaus in die Bandscheibe und endet dort mit einem Knäuel.
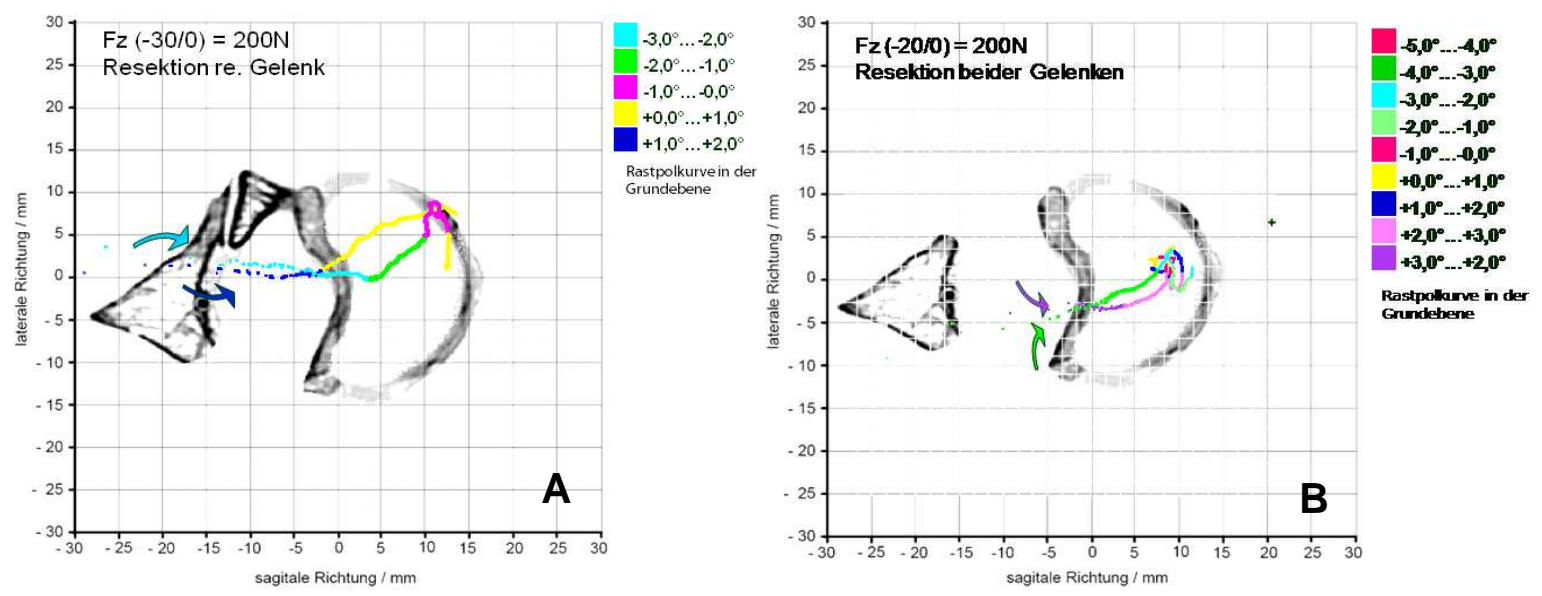

Abb. 3.2.15 A/B: Rastpolkurven von Th2/3 A. Durchstoßpunkte $R_{i}(\alpha)$ der momentanen Schraubachsen bei Vorlastposition dorsal nach Resektion des rechten Gelenks (A) und nach Resektion beider Gelenke (B). Die gebogenen Pfeile geben die Laufrichtung der Rastpolkurve und die Rotationsrichtung des oberen Wirbels an.

\section{Drehwinkel-Drehmoment-Kennlinien $\alpha(T)$}

Die Form der $\alpha(T)$ Funktion für die unterschiedliche Positionen der Vorlast ist annähernd sigmoid (Abb. 3.2.16). Eine Veränderung des Drehmoments am Anfang und Ende eines Messzyklus resultiert in einer geringen Auslenkung $\alpha$. Im Einzelnen zeigt sich eine deutliche Reduktion des Bewegungsumfangs für die dorsalen Vorlastpositionen $\left(\mathrm{x}_{\mathrm{F}}<0\right)$ von $|\alpha|=6^{\circ}$ (für zentrale Vorlast) auf $|\alpha|=3,75^{\circ}$ und eine geringe Reduktion für die ventrale Position von $F_{Z}\left(x_{F}>0\right)$ auf $|\alpha|=5,75^{\circ}$.

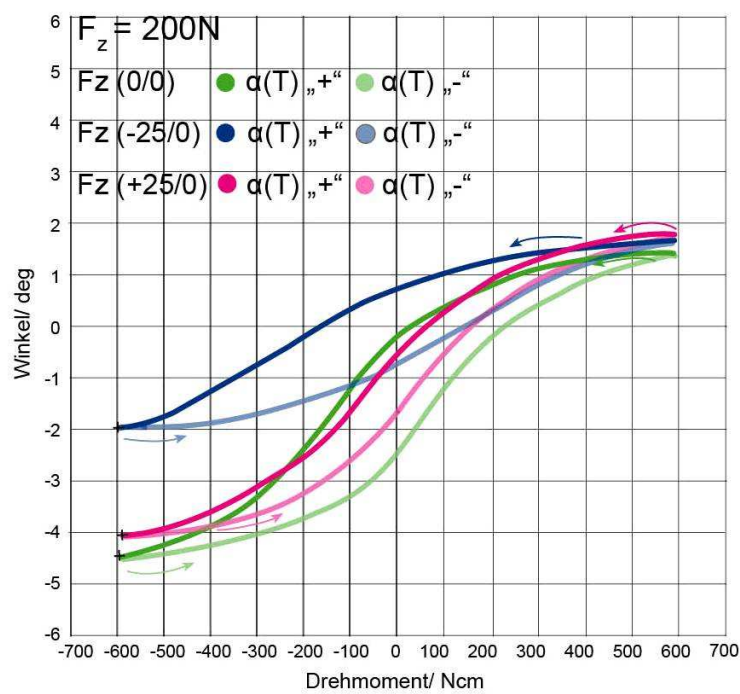

Abb. 3.2.16: Drehwinkel-DrehmomentKennlinien. Axialrotation $200 \mathrm{~N}$ in WZ: grün $F_{Z}(20 / 0)$, pink $F_{Z}(+20 / 0)$, blau $F_{Z}$ $(-20 / 0)$ 


\section{Rotationssteifigkeit $\mathrm{B}_{\mathrm{G}}(\alpha)$}

Die differentielle Steifigkeit bei Applikation einer ventralen Kraft (Flexion) unterscheidet sich nur unwesentlich von der Situation nach einem Kraftangriff im Widerstandszentrum

(Abb. 3.2.17). Applikation von dorsalen Vorlasten (Extension) erzeugt eine weit höhere anfängliche Rotationssteifigkeit des Segments (>740 Ncm/ deg). Gleichzeitig vergrößert sich auch die minimale Steifigkeit. Der versteifende Effekt ist stärker als bei einer Vervierfachung der zentralen Vorlast (Abb. 3.2.8, S. 84).

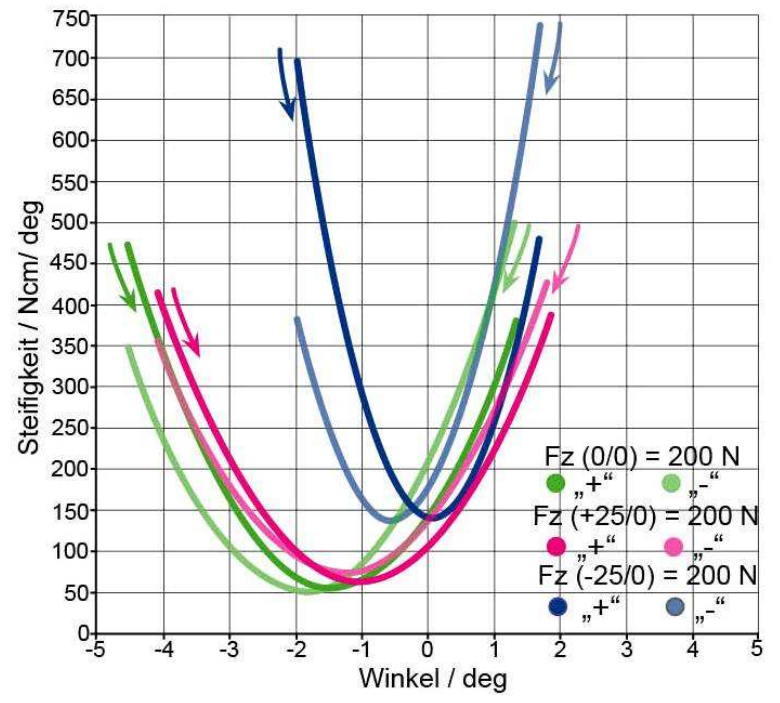

Abb.3.2.17: Die Rotationssteifigkeit $B_{G}(\alpha)$ in Abhängigkeit des Drehwinkels. Axialrotation $200 \mathrm{~N}$ Vorlast, grün: in WZ, pink: $25 \mathrm{~mm}$ ventral, blau: $25 \mathrm{~mm}$ dorsal.

\section{Sukzessive Resektion der Articulationes zygapophysiales Th2/Th3 A}

Nach sukzessiver Resektion von erst rechten, dann linken Wirbelbogengelenke zeigt sich auch hier eine symmetrische $\alpha(T)$-Funktion. Nach der ersten Resektion erhöht sich der Bewegungsumfang geringfügig von $|\alpha|=6^{\circ}$ auf $|\alpha|=6,2^{\circ}$. Diese Beobachtung gilt für alle betrachteten Vorlastpositionen. Nach Resektion der zweiten Art. zygapophysialis wird wieder eine symmetrische $\alpha(T)$-Abhängigkeit mit einem weit größeren Rotationsbereich von $|\alpha|=8,5^{\circ}$ beobachtet (Abb. 3.2.18).

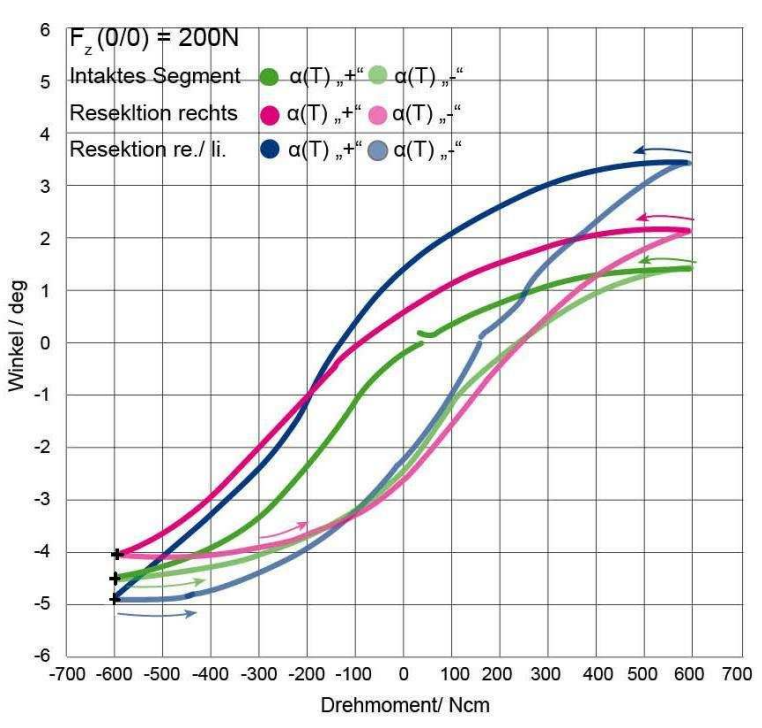

Abb. 3.2.18: Drehmoment-Drehwinkel-Kennlinie nach Resektion der Gelenke. 
Die differentielle Steifigkeit bei der Resektion erst rechten und danach linken Art. zygapophysialis bleibt annähernd gleich (Abb. 3.2.19).

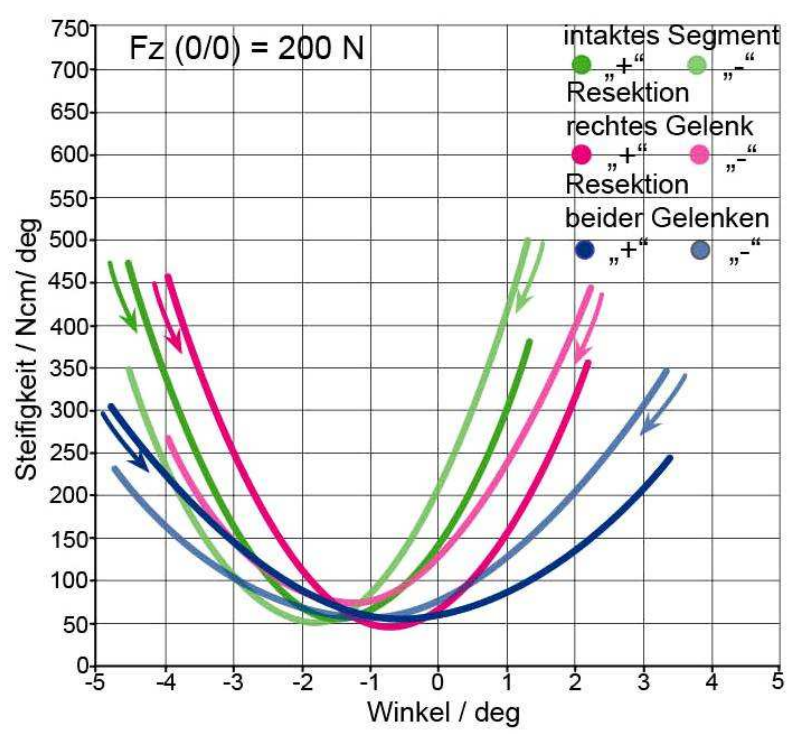

Abb.3.2.19: Die Rotationssteifigkeit $B_{G}(\alpha)$ in Abhängigkeit des Drehwinkels.

\section{Richtung der momentanen Schraubachsen e ( $r$ )}

Die Verschiebung der Vorlast in sagittaler Richtung nach dorsal verändert das Verhalten des Richtungsvektors der $\mathrm{IHA}\left(\mathrm{T}_{\mathrm{z}}\right)$.

In der Darstellung der einzelnen Komponenten des normierten Richtungsvektors $\underline{\mathrm{e}}$ der $\mathrm{IHA}\left(\mathrm{T}_{\mathrm{Z}}\right)$ für eine Vorlastposition von $25 \mathrm{~mm}$ dorsal des Widerstandszentrums steigt die ey-Komponente rapide an (Abb. 3.2.20, S. 94). Die Schraubachse schwenkt in der Frontalebene bei der Linksdrehung (positive Rotationsrichtung) von rechts nach links und bei der Rechtsdrehung umgekehrt von links nach rechts. Bei maximaler Linksdrehung ist $e_{Y}=0,25$ und $e_{Z}=0,95$, was einem Neigungswinkel von $75,3^{\circ}$ nach links-lateral entspricht. Somit verkippt die momentane Schraubachse um $14,7^{\circ}$ von der senkrechten Stellung nach links.

Die $e_{\mathrm{x}}$-Komponente hat bei einer Linksdrehung über den gesamten Rotationsbereich stets einen positiven Wert und für Rechtsdrehung stets einen negativen Wert, was einer Verkippung der IHA während der Rotation nach ventral entspricht. Damit ist die Schraubachse bei einem dorsalen Kraftangriff in der maximalen Linksdrehung um $14,7^{\circ}$ nach links-lateral und in der maximalen Rechtsdrehung nach um $10,4^{\circ}$ rechts-lateral und gleichzeitig um $7,5^{\circ}$ nach ventral gekippt. 
Bei der ventralen Belastung von $200 \mathrm{~N} \mathrm{~F}_{Z}(+25 / 0)$ ist der Verlauf der $e_{Y^{-}}$ Komponente umgekehrt (Abb. 3.2.21). Während einer Rechtsdrehung kommt es zur lateralen Verkippung von rechts nach links.

Es kommt zu einer neuen Ausrichtung der $e_{X}$-Komponente. Das Verhalten ist umgekehrt, so verkippt die IHA während der Rotation nach dorsal.

Bei ey-Komponente kommt es auch zur Richtungsumkehr. Bei der maximalen Linksdrehung verkippt die IHA um 18,6 nach rechts- lateral und bei Rechtsdrehung um 14,7 nach links-lateral in der Frontaleben e.

Gleichzeitig verkippt die momentane Schraubachse während der Gesamtrotation leicht nach dorsal um $1,8^{\circ}($ Abb.3.2.21).

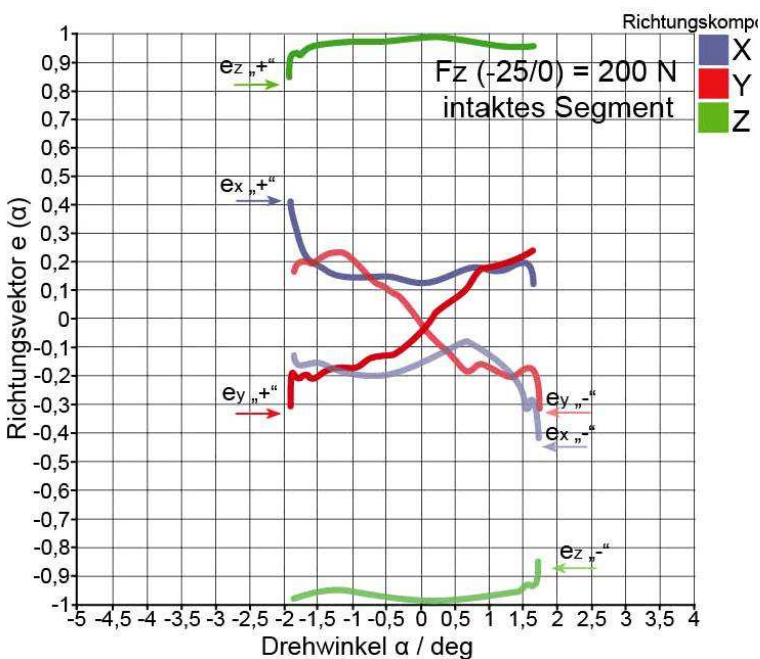

Abb. 3.2.20: Richtungsvektor $\underline{e}$ der momentanen Schraubachsen in Abhängigkeit von $\alpha$ für eine dorsale Vorlastposition $\mathrm{Fz}(-25 / 0)$.

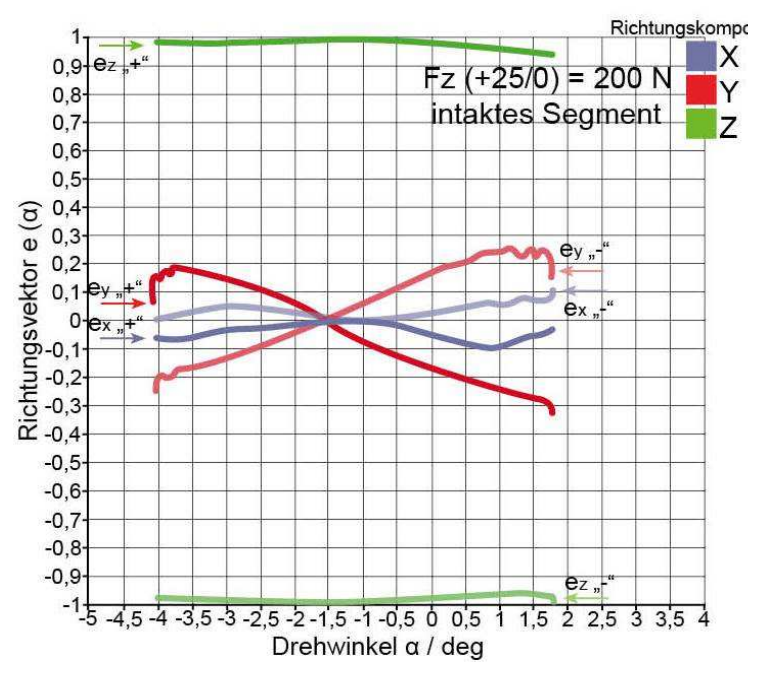

Abb. 3.2.21: Richtungsvektor $\underline{e}$ der momentanen Schraubachsen in Abhängigkeit von $\alpha$ für eine ventrale Vorlastposition $\mathrm{Fz}(+25 / 0)$.

\section{Sukzessive Resektion der Articulationes zygapophysiales von Th2/Th3 A}

Die Resektion des rechten Bogengelenks führt zur lateralen Verkippung der momentanen Schraubachse (Abb. 3.3.22, S. 95). Eine laterale Verkippung der momentanen Schraubachse in der maximalen Linksdrehung ist gering und beträgt für $e_{Y}=0,09$ und $e_{z}=0,99$ etwa $5,7^{\circ}$ bei einem Neigungswinkel von $84,3^{\circ}$ zur linken Seite. Nach Ausrichtung von $e_{x}$-Komponente (positiv bei einer Linksdrehung über den gesamten Rotationsbereich und negativ für Rechtsdrehung) ist die momentane Schraubachse während der Gesamtrotation um max. 8,6nach ventral gekippt. 
Nach dem Entfernen von der verbleibenden Art. zygapophysialis ist die maximale $e_{Y}-K o m p o n e n t e ~ l e ~ l=0,3$ (entsprechend einem Winkel von $\theta_{Y}=16,8^{\circ}$ ) (Abb. 3.3.23). Die momentane Schraubachse verkippt während der Gesamtrotation um 11,4 nach ventral.

Bei der sukzessiven Resektion erst rechten, dann linken Art. zygapophysialis kommt es zu einer immer stärkeren Verkippung der momentanen Schraubachse sowohl nach links und rechts, als auch nach ventral.

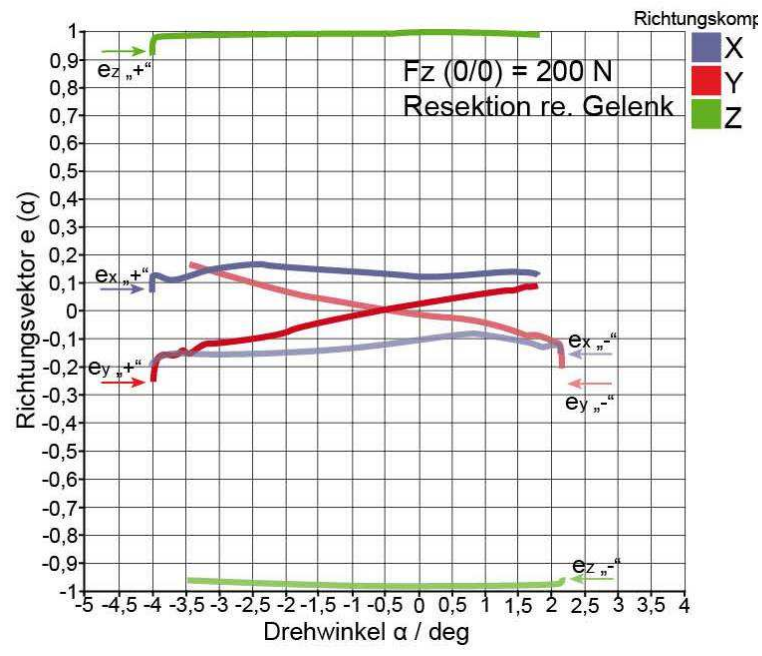

Abb. 3.2.22: Richtungsvektor der momentanen Schraubachse. Die Komponenten des Richtungsvektors von Th 2/3 A nach Resektion des rechten Bogengelenks.

\section{Schraubsteigung}

Die Abbildung 3.2.24 zeigt die Verschraubung $\tau$ des oberen Wirbelkörpers entlang der momentanen Schraubachse in der Einheit $\mathrm{mm} /$ Grad in Abhängigkeit des Drehwinkels $\alpha$. Für die Drehwinkeln $\alpha$ in der Rechtsdrehung für $\mathrm{F}_{\mathrm{Z}}(0 / 0)$ bei konstanter Vorlast von $200 \mathrm{~N}$ ist die Abhängigkeit näherungsweise linear. Mit ansteigendem Drehwinkel nimmt die Schraubsteigung zu, die

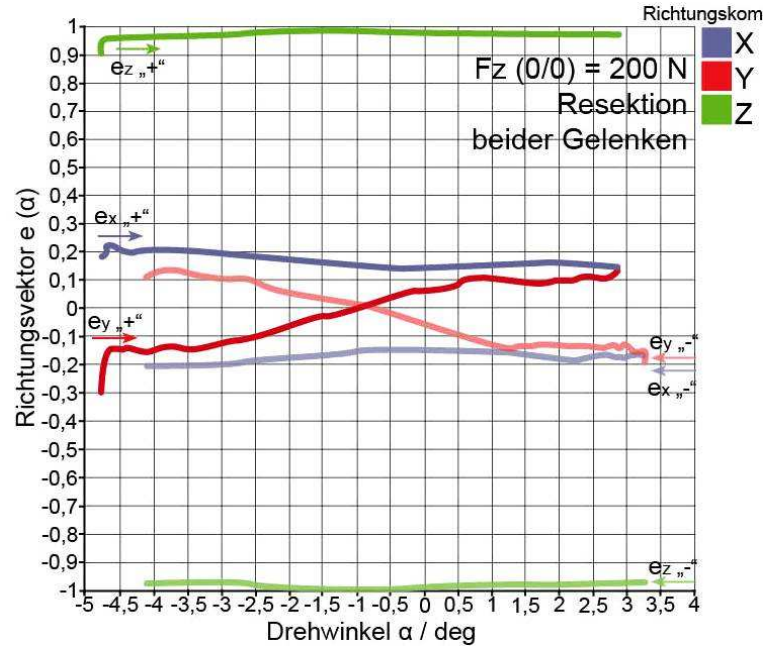

Abb. 3.2.23: Richtungsvektor der momentanen Schraubachse. Die Komponenten des Richtungsvektors von Th 2/3 A nach Resektion von beiden Bogengelenken.

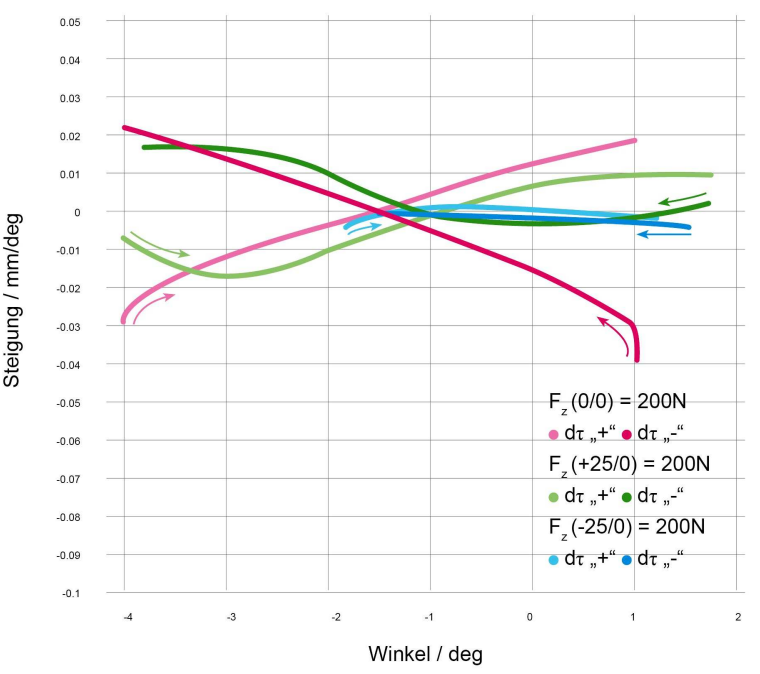

Abb. 3.2.24: Schraubsteigung $\tau(\alpha)$ der momentanen Schraubachsen von Th2/3 A bei verschiedenen Vorlastpositionen. 
Bandscheibe elongiert und die Wirbelkörper entfernen sich voneinander. Bei einer Linksdrehung ist die Abhängigkeit umgekehrt proportional der Rechtsdrehung und die Schraubsteigung nimmt mit steigendem Drehwinkel ab. Die Bandscheibe wird gestaucht. Für Hin- und Rücklauf innerhalb eines Messzyklus für $\alpha=1,5^{\circ}$ ist die Schraubsteigung gleich Null.

Die berechneten Werte für $F_{Z}(0 / 0)$ liegen zwischen $-0,04 \mathrm{~mm} /$ Grad für die Stauchung und 0,022 $\mathrm{mm} /$ Grad für eine Dehnung der Zwischenwirbelscheibe.

Die qualitativen Unterschiede sind bei ventralen und dorsalen Vorlastpositionen zu verzeichnen. Für eine dorsale Vorlast $\left(\mathrm{x}_{\mathrm{F}}<0\right)$ ist die maximale Schraubsteigung mit $0,001 \mathrm{~mm} / \mathrm{Grad}$ deutlich kleiner im Vergleich zu $0,018 \mathrm{~mm} / \mathrm{Grad}$ bei einer ventral positionierten Vorlast $\left(\mathrm{x}_{\mathrm{F}}>0\right)$ (Abb. 3.2.17, S. 95). Die Schraubsteigung für dorsale Vorlast mit einem reduzierten Rotationswinkelbereich von $\alpha= \pm 1,6^{\circ}$ zeigt dementsprechend auch die kleinere Steigung $\tau(\alpha)$ von $-0,015$ bis $0,001 \mathrm{~mm} / \mathrm{Grad}$. Für $\mathrm{X}_{F}>0$ ist der maximale Rotationsbereich größer und liegt bei 6,8. Zu Beginn der Linksdrehung kommt es zur einen Abwärtsbewegung des Segments bis $\alpha \approx$ $0^{\circ}$, wo es in eine Aufwärtsbewegung des oberen Wirbe ls umschlägt (negative Drehwinkel). Bei der Rechtsdrehung ist diese Abfolge umgekehrt. Die maximale Schraubsteigung liegt zwischen $-0,018$ und $0,017 \mathrm{~mm} / \mathrm{Grad}$.

\section{Sukzessive Resektion der Articulationes zygapophysiales von Th2/Th3 A}

Nach der Entfernung des rechten Bogengelenks zeigt die Schraubsteigung $\tau(\alpha)$ einen asymmetrischen Verlauf. Bei der Linksdrehung ist die Schraubsteigung für den kompletten Drehwinkelbereich von $-4^{\circ}$ bis $+2^{\circ}$ gleich Null. Es kommt weder zu einer Abwärts- noch zu einer Aufwärtsbewegung des oberen Wirbels (Abb. 3.2.25). Bei der Rechtsdrehung ist ein Auseinander-

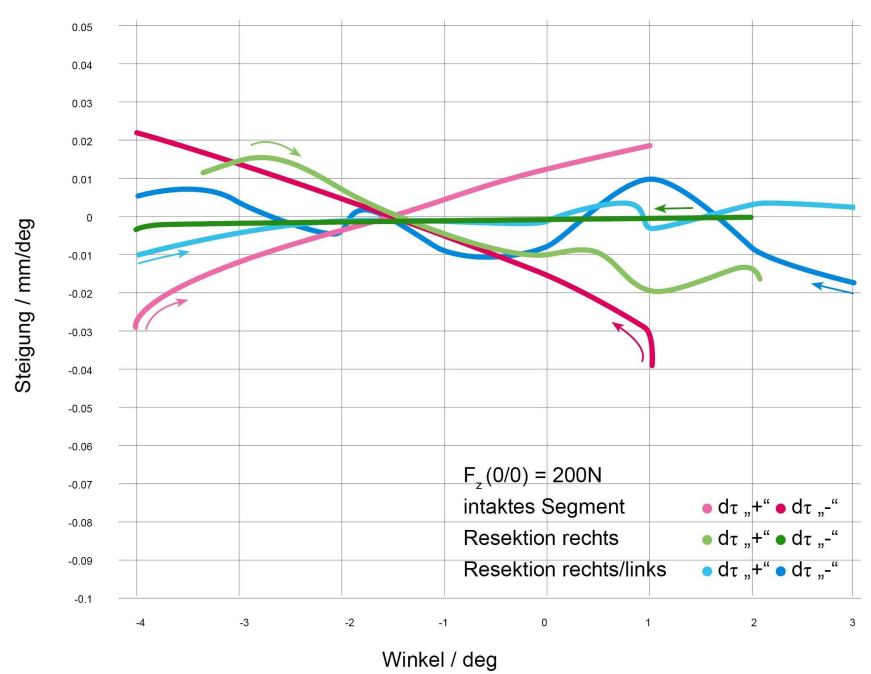

Abb. 3.2.25: Schraubsteigung $\tau(\alpha)$ der momentanen Schraubachse nach Resektion des rechten Gelenks und dann beider Gelenke. 
weichen beider Wirbel zu verzeichnen. Die Schraubsteigung nimmt für $\alpha>-3^{\circ} u m$ $0,025 \mathrm{~mm}$ ab.

Die Resektion des zweiten Gelenks resultiert in einer annähernd ebenen Bewegung (entartete Verschraubung mit $\tau \pm 0,01$ ) für Rotationswinkel $|\alpha|<3^{\circ}$. Erst bei einer extremen Auslenkung $\alpha$ steigt $\tau(\alpha)$ leicht an.

\subsubsection{Verschiebung der axialen Vorlast entlang der y-Achse;}

$F Z\left(X_{F} / y_{F}\right) ; I F_{Z} I=200 N ; \underline{T}(t)=\left(0,0, I_{Z}(t)\right)$

Die Vorlast $F_{Z}=200 N$ wird entlang der $y$-Achse im Widerstandszentrum $W_{Y}(0 / 0)$ verschoben (3.2.26 A, B). Es resultiert eine laterale Verkippung des oberen Wirbelkörpers nach links $\left(y_{F}>0\right)$ bzw. nach rechts $\left(y_{F}<0\right)$.

Q $\mathrm{Fz}\left(\mathrm{X}_{F} / \mathrm{yF}_{\mathrm{F}}\right)=200 \mathrm{~N}$
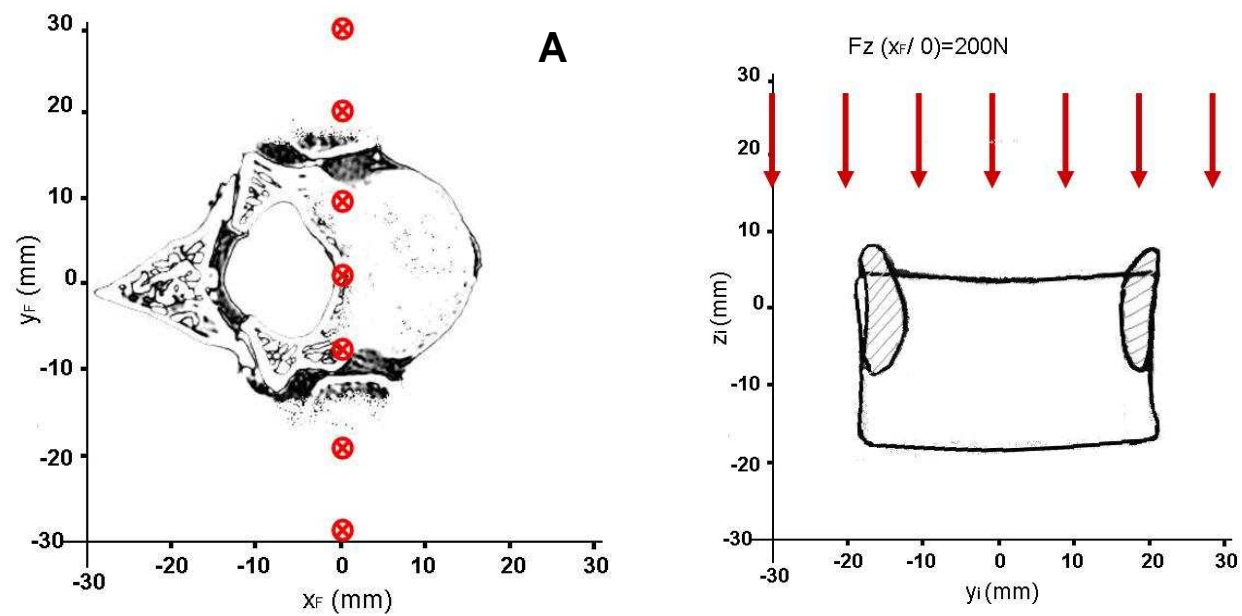

B

Abb. 3.2.26 A/B: Auswertung statischen Messungen für beide Bewegungssegmente (Th2/3 A und $B)$. Durchstoßpunkte der Kraftwirkungslinie von $\mathrm{F}_{Z}\left(\mathrm{x}_{\mathrm{F}} / 0\right)$ durch die $x y$-Referenzebene von kranial betrachtet $(\mathbf{A})$. Position der Kraftwirkungslinie $\mathrm{F}_{Z}\left(\mathrm{x}_{\mathrm{F}} / 0\right)$ in der Medianebene von ventral betrachtet (B).

\section{Rastpolkurven $r(\alpha)$}

Die Rastpolkurve für Vorlastpositionen $-10 \mathrm{~mm} \leq \mathrm{y}_{\mathrm{F}}<+10 \mathrm{~mm}$ verändert sich nicht wesentlich. Erst bei einer Verschiebung von $\mathrm{F}_{Z}$ um mehr als $20 \mathrm{~mm}$ kommt es zur qualitativen Veränderung. Zum Ersten resultiert eine Verlagerung der Rastpolkurven zur Seite der jeweiligen Belastung. Und zum Zweiten zeigt sich ein deutlicher Unterschied zwischen einer Rechts- und Linksdrehung des oberen Wirbels inner- 
halb eines Messzykluses. Der Rotationsumfang $\alpha(\mathrm{T})$ ist je nach Richtung der Lateralflexion reduziert. Eine Lateralverkippung des Wirbelkörpers um $\kappa_{x}>0$ durch eine axiale Kraft mit $y_{F}$ von -10 bis - $30 \mathrm{~mm}$ führt zu einer Reduktion der Rotation in positive Richtung mit $\alpha(T)=-2,5^{\circ} \rightarrow 2,5^{\circ}$. Bei Lateralflexion um $\kappa_{x}<0$ durch axiale Kraft mit Ansatz am $\mathrm{y}_{\mathrm{F}}>10 \mathrm{~mm}$ resultiert ein reduzierter Rotationsumfang $\alpha(\mathrm{T})$ in negative Richtung mit $\alpha(T)=-5,0^{\circ} \rightarrow 4^{\circ}$ (Abb. 3.2.27 A/B).
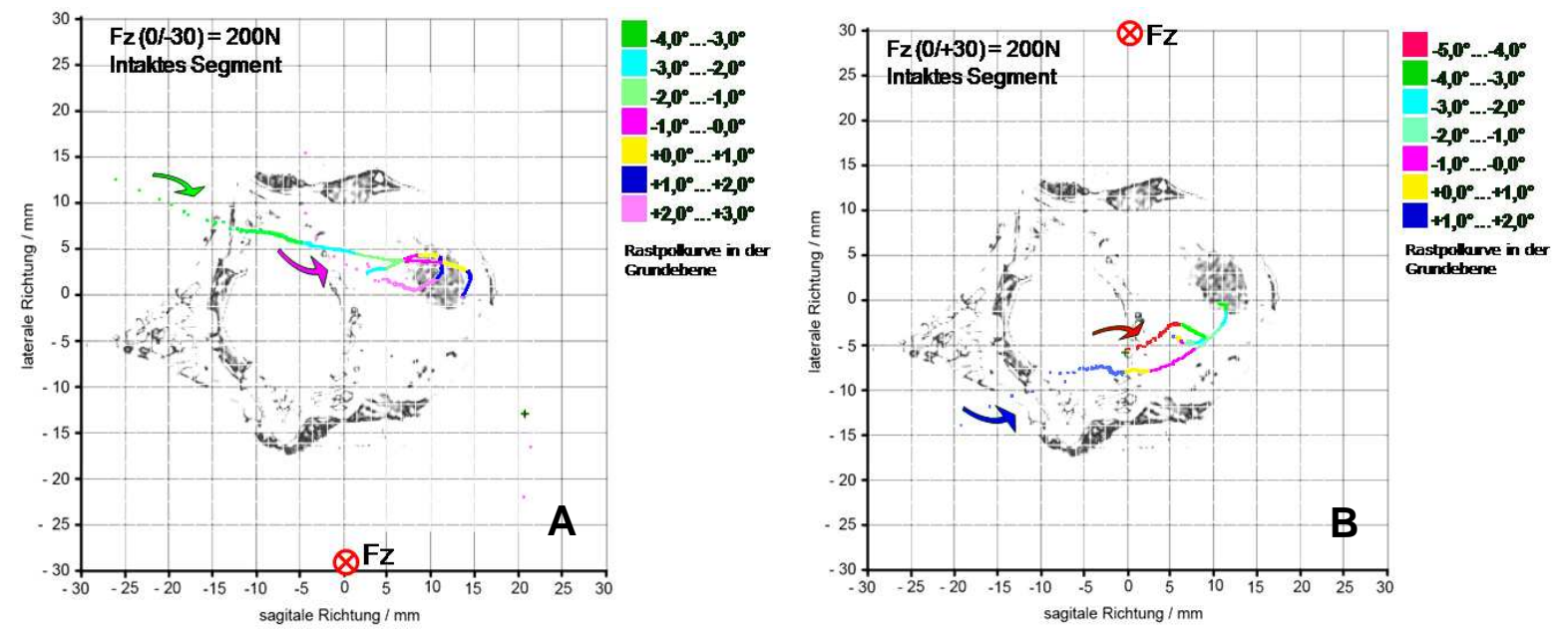

Abb. 3.2.27 A/ B: Rastpolkurven von Th2/3 A.

Durchstoßpunkte $\mathrm{R}_{\mathrm{i}}(\alpha)$ der momentanen Schraubachsen bei Vorlastposition in $\mathrm{y}_{\mathrm{F}}=-30 \mathrm{~mm}(\mathbf{A})$ und bei einer Vorlastposition in $\mathrm{y}_{\mathrm{F}}=+30 \mathrm{~mm}(\mathbf{B})$. Die gebogenen Pfeile geben die Laufrichtung der Rastpolkurve und die Rotationsrichtung des oberen Wirbels an.

\section{Sukzessive Resektion der Articulationes zygapophysiales von Th2/Th3 A}

Weiteren Aufschluss über das Führungsverhalten der einzelnen Wirbelbogengelenke gibt zunächst die einseitige Resektion des rechten Gelenks von Th 2/3 A. Die Rastpolkurve bei der lateralen Vorlastposition $(0 /+30)$ und nach einseitiger Resektion der rechten Art. zygapophysialis verlagert sich zur Seite des vorhandenen Gelenks. Die Rechtsdrehung entspricht nur halbseitig der gleichnamigen Rastpolkurve des intakten Segments. Die momentanen Schraubachsen $\operatorname{dr}(\alpha)$ bei maximaler Auslenkung - $\alpha$ liegen größtenteils in der linken Hälfte der Bandscheibe und wandern für eine Rotationsbewegung in positive Richtung (gegen den Uhrzeigersinn in Aufsicht) von dorsal nach ventral (Abb.3.2.28 A, S. 99). 

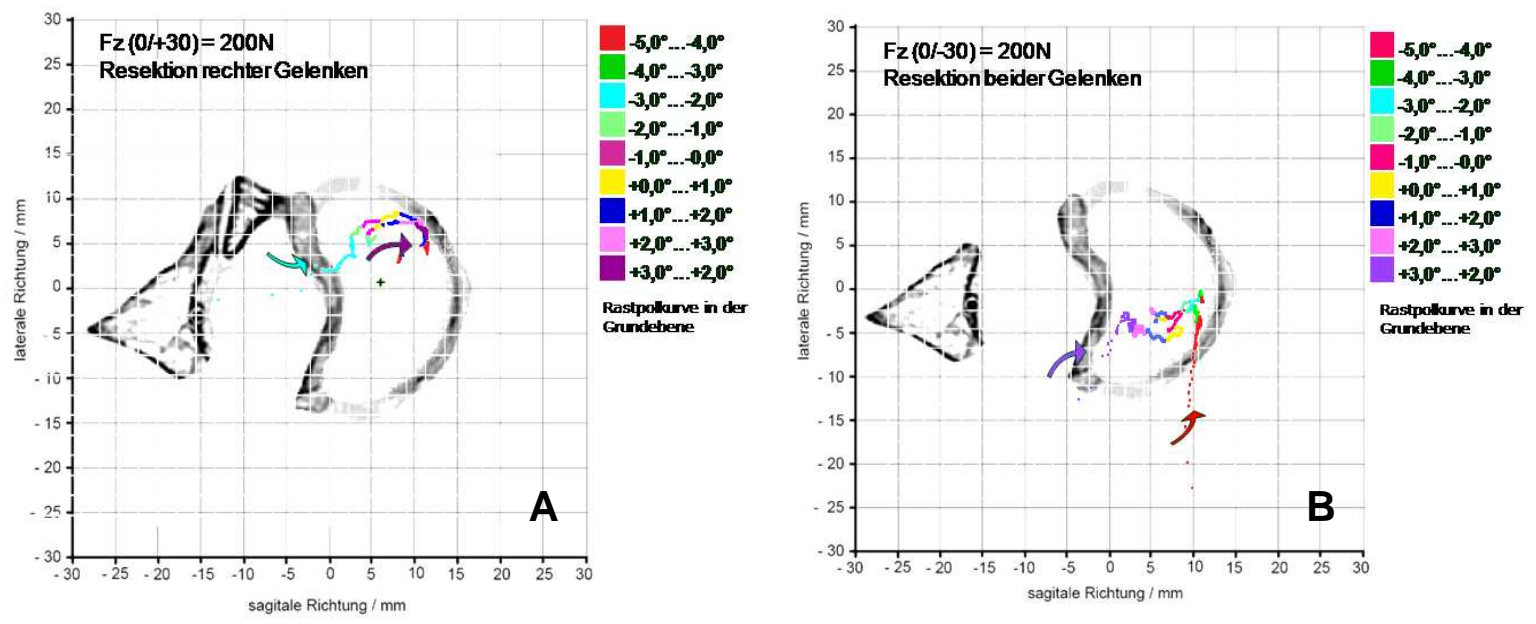

Abb. 3.2.28 A/ B: Rastpolkurven von Th2/3 A.

Durchstoßpunkte $R_{i}(\alpha)$ der momentanen Schraubachsen bei Vorlastposition in $y_{F}=+30 \mathrm{~mm}$ nach Resektion rechten Wirbelbogengelenkes $(\mathbf{A})$ und bei Vorlastposition in $\mathrm{y}_{\mathrm{F}}=-30 \mathrm{~mm}$ nach Resektion beider Wirbelbogengelenken (B). Die gebogenen Pfeile geben die Laufrichtung der Rastpolkurve und die Rotationsrichtung des oberen Wirbels an.

Die Abbildung 3.2.28 B zeigt die momentanen Schraubachsen $\mathrm{dr}(\alpha)$ nach beidseitiger Entfernung der Artt. zygapophysiales. Die Rastpolkurven verknäueln sich etwas rechts der Bandscheibenmitte. Der Rotationsumfang $\alpha(T)$ ist je nach Richtung der Lateralflexion reduziert.

In Abbildung 3.2.28 B zeigt die Rastpolkurve eine komplett andere Position und Verlauf als in Abbildung A.

Die Steifigkeit der axialen Rotation nimmt bei vorhergegangener lateralen Verkippung des oberen Wirbelkörpers ab. Die Richtungskomponenten der Schraubachsen, insbesondere die größere y-Komponente des Richtungsvektors $\mathrm{e}(\alpha)$ und die momentane Schraubsteigung $\tau(\alpha)$, korrelieren mit dem einseitigen Anstieg der Steifigkeit. Der Verlauf der momentanen Schraubsteigung $\tau(\alpha)$ und der $y$ Komponente des Richtungsvektors $e_{Y}(\alpha)$ zeigen ein lineares Verhalten. 


\subsubsection{Bewegungssegment Th2/3 B}

Das zweite Junghannssche Segment Th2/3 B wurde leider im Laufe der Messung beschädigt. Nach Resektion des rechten Wirbelbogengelenks und beim Aufbau von nachfolgendem Experiment am Bewegungssegment kam es zu einem Riss des Ligamentum intertransversarium und der Gelenkkapsel an der verbleibenden Art. zygapophysialis. Wie gefolgt ist die vorliegende Untersuchung auf Axialrotation, Lateralflexion und Flexion/Extension nur auf intaktes Segment Th2/3 B beschränkt.

\subsubsection{Rotation ohne axiale Vorlast; $\mathrm{T}(\mathrm{t})=(0,0, \mathrm{Tz}(\mathrm{t})), \mathrm{F}=(0,0,0)$}

\section{$\underline{\text { Rastpolkurven }}$}

In Abbildung 3.2.29 setzt die Rechtsdrehung aus einer Extremposition mit $\alpha=-4^{\circ}$ ein. Die momentane Schraubachse liegt im Bereich der linken Art. Zygapophysialis. Die Regelfläche beschreibt einen nach ventral konvexen Bogen. Bei Änderung der Drehrichtung springt die Rastpolkurve zur Gegenseite und wandert ebenso nach ventral in einem konvexen Bogen.

Die Schraubachsen sind also nicht

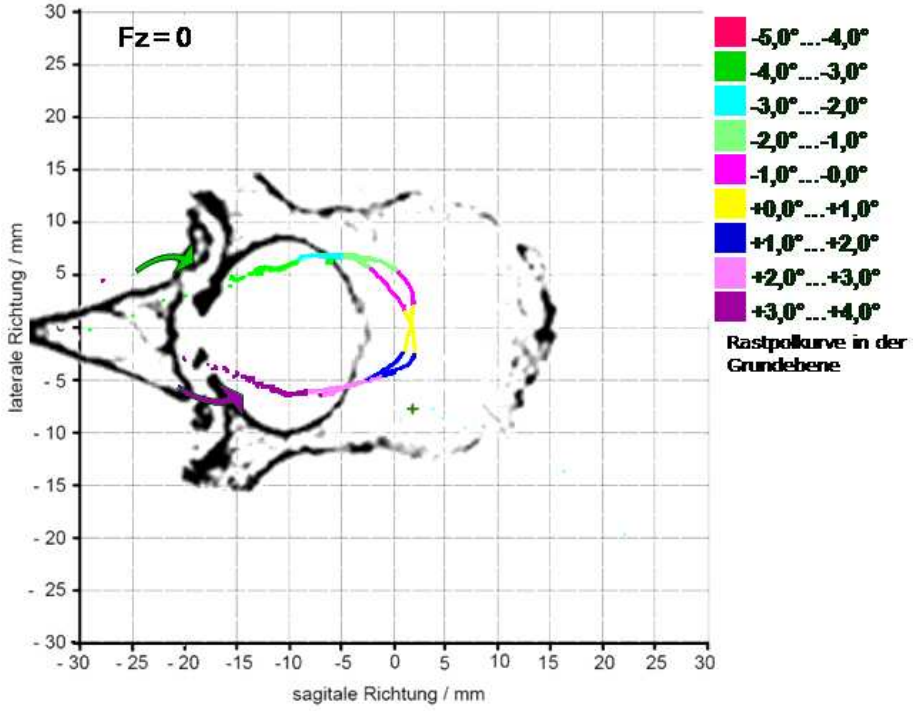

Abb. 3.2.29 (Th2/3 B): Ansicht von kranial. Durchstoßpunkte $R_{i}(\alpha)$ der momentanen Schraubachsen für eine Drehung in positive und negative Richtung $\left(\mathbf{F}_{\mathbf{Z}}=\mathbf{0}\right)$. ortsfest. Die Bewegung ist eben und gleichmäßig. 
Drehwinkel-Drehmoment-Kennlinie

Es wurde ein zyklisches Drehmoment $\mathrm{T}(\mathrm{t})$ appliziert. Der Bewegungsumfang (ROM) des zweiten Segments Th2/3 B bei dem gleichbleibenden maximal angreifenden Drehmoment von $600 \mathrm{~N} / \mathrm{cm}$ ist wesentlich größer und beträgt $7^{\circ}$ (Abb. 3.2.30). Der Hin- und Rücklauf eines Messzykluses fallen nicht zusammen.

Es kann eine deutliche Hysterese $\Delta \alpha(T=0)$ gemessen werden. Sie beträgt für das unbelastete Segment bei $F_{Z}=0$

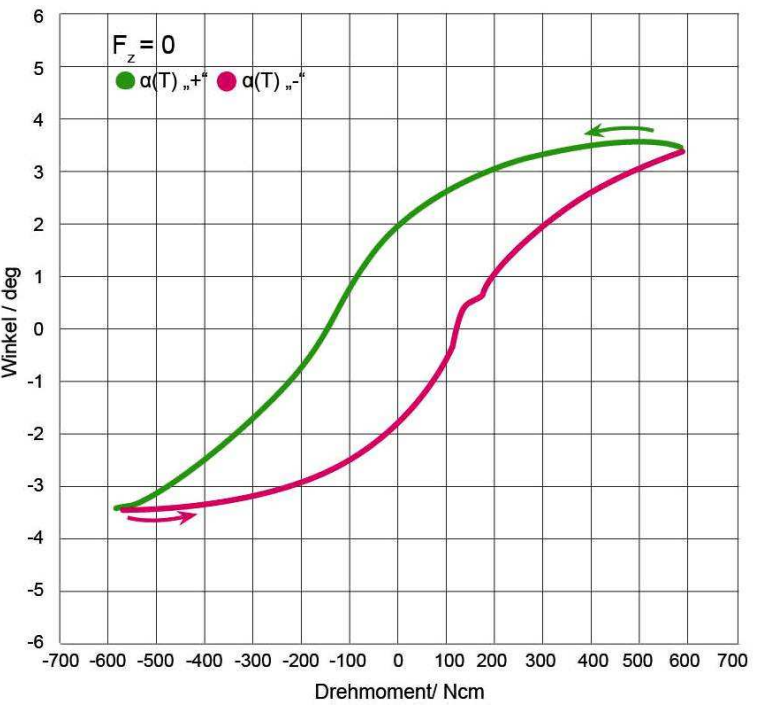
Abb. 3.2.30: Drehmoment-DrehwinkelKennlinie; die Pfeile zeigen die Laufrichtung an. $3,8^{\circ}$.

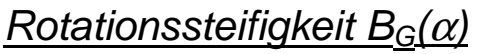

Der Verlauf der Gesamtsteifigkeit $\mathrm{B}_{\mathrm{G}}(\alpha)$ zeigt eine hohe anfängliche Rotationssteifigkeit $(>440 \mathrm{Ncm} / 9$ ) und eine Erhöhung am Ende eines Halbzykluses $\left(B_{G} \cong 350 \mathrm{Ncm} /{ }^{\circ}\right)$. Für $\alpha=0^{\circ}$ beträgt sie $35 \mathrm{Ncm}^{\circ}{ }^{\circ}$ (Abb. 3.2.31).

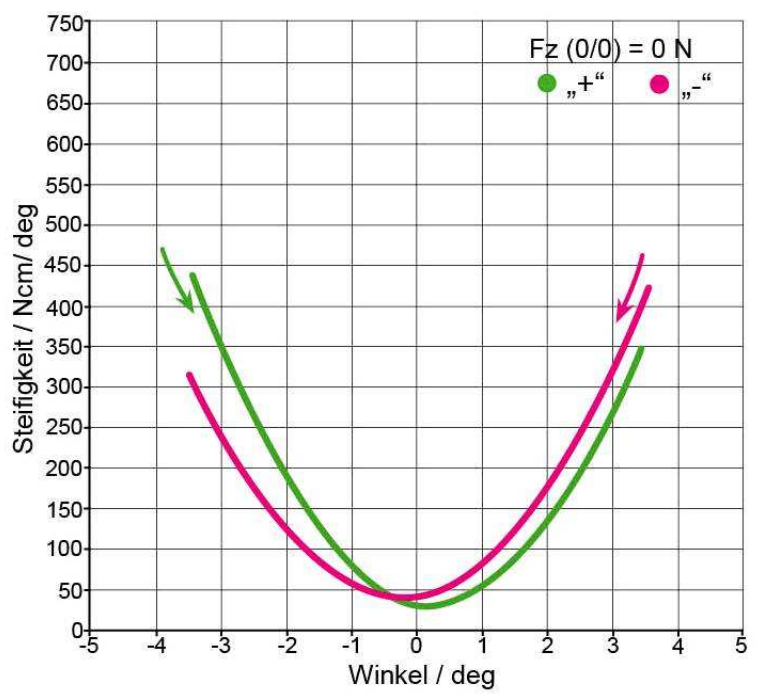

Abb.3.2.31: Die Rotationssteifigkeit $B_{G}(\alpha)$ in Abhängigkeit des Drehwinkels. 
Richtungsvektor der Schraubachse e $(\alpha)$

Die Abbildung 3.2.32 zeigt das Verhalten der einzelnen Komponenten des Einheitsvektors $\mathrm{e}(\alpha)$ der momentanen Schraubachse.

Der Wert der z-Komponente liegt konstant bei $e_{z}(\alpha)=1$ für Rechts- und Linksdrehung. Die y-Komponente ist für positive Drehwinkel $\alpha$ von $0^{\circ}$ bis $3^{\circ}$ weitgehend gleich 0 , so richtet sich die momentane Schraubachse parallel zur Drehachse. Bei den negativen Drehwinkeln verkippt die IHA zunehmend nach rechts. In der maximalen Rechtsdrehung sind $\mathrm{e}_{\mathrm{Y}}=0,1^{\circ}$ und $\mathrm{e}_{\mathrm{X}}=$ $1^{\circ}$, was einer Verkippung um $5,7^{\circ}$

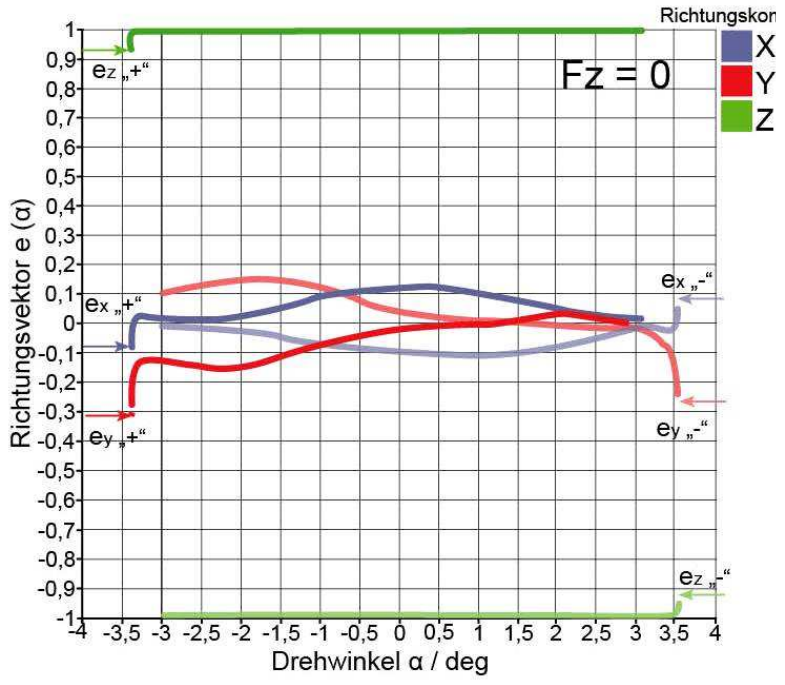

Abb. 3.2.32: Die Komponente des Richtungsvektors $\underline{e}$ (Th2/3 A) in Abhängigkeit des Drehwinkels $\alpha$. nach rechts-lateral entspricht. In der maximalen Linksdrehung richtet sich die IHA wieder nach links auf und stellt sich parallel zur Rotationsachse. $E_{Y}(\alpha)$ erreicht maximal $0,15^{\circ}$, was einem Neigungswinkel $\theta_{y}$ der Schraubachse von $8,5^{\circ}$ entspricht. Die $e_{x}$-Komponente hat bei einer Linksdrehung über den gesamten Rotationsbereich stets einen positiven Wert und bei der Rechtsdrehung stets einen negativen Wert, was einer Verkippung der IHA während der Rotation nach ventral entspricht.

Damit ist die Schraubachse bei axialer Rotation eines unbelasteten Segments in der maximalen Linksdrehung parallel zur z-Achse ausgerichtet, und in der maximalen Rechtsdrehung um 5,7\% nach rechts-lateral und in Laufe des kompletten Messzykluses maximal um $6,8^{\circ}$ nach ventral gekippt.

\section{Schraubsteigung $\tau(\alpha)$}

Es kommt zu einem Versatz ds entlang der differentiellen Schraubachse $\operatorname{dr}(\alpha)$. Die Bewegung für Hin- und Rücklauf ist spiegelsymmetrisch. 
Zu Beginn der Linksdrehung (negative Richtung) bewegt sich das obere Segment nach oben um 0,04mm (Abb. 3.2.33). Die gewonnene Höhe bleibt bis zu dem Drehwinkel $\alpha=-2,3^{\circ}$ unverändert. Ihr folgt ein linearer Anstieg des Versatzes ds entlang der differentiellen Schraubachse $\operatorname{dr}(\alpha)$ und die Wirbelkörper entfernen sich voneinander. Die Bandscheibe wird mit $S_{\max }=0,12 \mathrm{~mm}$ leicht elongiert. Bei der

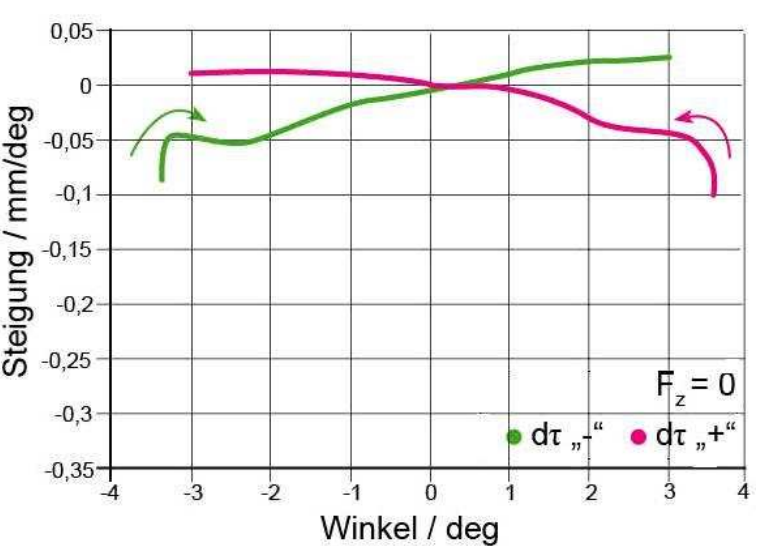

Abb. 3.2.33: Die momentane Schraubsteigung $\tau(\alpha)$ in Abhängigkeit des Drehwinkels $\alpha$ für ein kompletten Messzyklus.

Rechtsdrehung ist die Abfolge gleich bei umgekehrter Rotationsrichtung. Die Bandscheibe elongiert geringfügig um den gleichen Betrag.

\subsubsection{Erhöhung des Betrags der axialen Vorlast}

$$
\underline{I}(\mathrm{t})=\left(0,0, \underline{T}_{z}(\mathrm{t})\right), \mathrm{F}=\left(0,0, F_{z}\right)
$$

\section{Rastpolkurven}

Der experimentelle Versuchsaufbau ist dem in Kapitel 3.2.1 (S. 78) gleich. Im Widerstandszentrum $W_{Y}$ (für Th2/3 B, 5mm ventral der Wannenmitte) wird die axiale Vorlast $\mathrm{F}_{Z}$ schrittweise um $100 \mathrm{~N}$ von anfangs $100 \mathrm{~N}$ auf $400 \mathrm{~N}$ erhöht und ein zyklisches Drehmoment $T_{Z}(t)$ appliziert. Die erhöhte Vorlast $F_{Z}$ verstärkt die Kompression der Bandscheibe und verändert den Kraftschluss der Gelenke.

Der Verkippungswinkel des

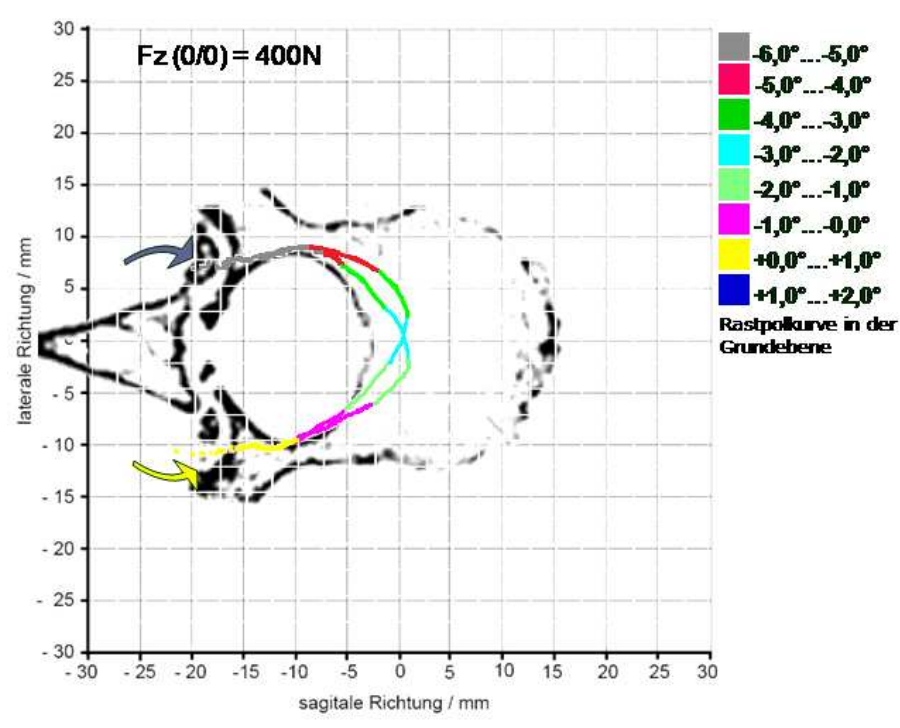

Abb. 3.2.34 (Th2/3 B): Ansicht von kranial.

Durchstoßpunkte $R_{i}(\alpha)$ des momentanen Schraubachsen für eine Drehung in positiver und negative Richtung $\left(F_{Z}=400\right)$. Die Pfeile geben die Laufrichtung der Rastpolkurve an. 
oberen Wirbels ist meist negativ, so schwenkte die momentane Schraubenachse für fast den kompletten Rotationsbereich nach dorsal in $\alpha=-6^{\circ} \rightarrow 0^{\circ}$ (Abb. 3.2.34). Bei dem Bewegungssegment $B$ verbleibt die Rastpolkurve in der Bandscheibenmitte, beult sich nur stärker aus. Eine Erhöhung der Vorlast von $100 \mathrm{~N}$ auf $400 \mathrm{~N}$ verändert $r_{i}(\alpha)$ nur leicht.

\section{Drehwinkel- Drehmoment- Kennlinie}

Das $\alpha(T)$-Diagramm verändert sich merklich in Abhängigkeit von der Größe der Vorlast. Für $\mathrm{F}_{Z}=400 \mathrm{~N}$ ist die gemessene Hysterese $\Delta \alpha=1,8^{\circ}$ etwas größer als für $F_{Z}=200 N$ mit $\Delta \alpha=1,2^{\circ}$, aber beide Hysterese sind deutlich kleiner als bei $F_{Z}=0 N$ (Abb. 3.2.30, S. 101).

Der Bewegungsumfang von $7^{\circ}$ bliebt annähernd gleich. Die Vorlastgröße beeinflusst nicht den maximalen Rotationsumfang. Die Tatsache aber, dass der Hystereseumfang des belasteten

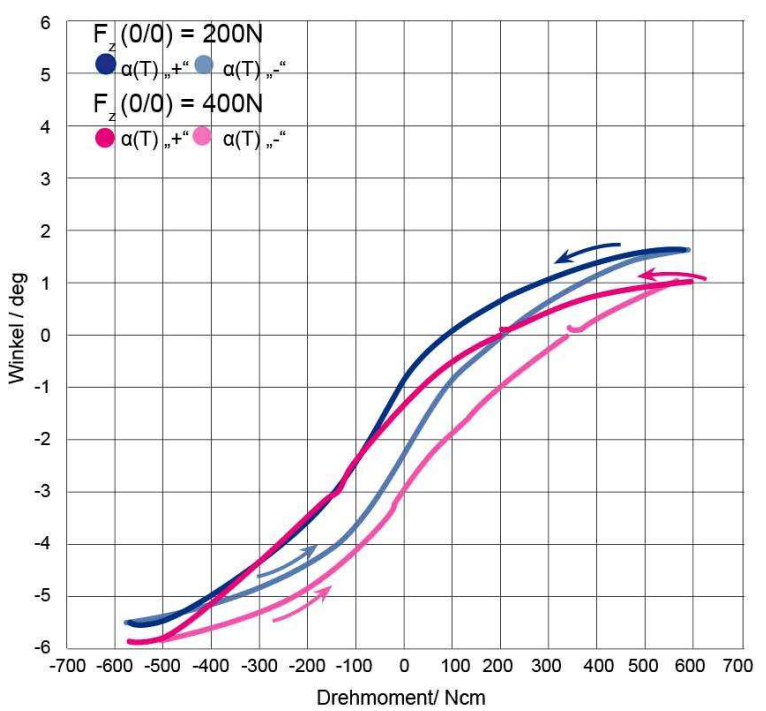

Abb. 3.2.35: Drehmoment-Drehwinkel Kennlinien. Axialrotation: blau $F_{Z}=200 \mathrm{~N}$, pink $F_{Z}=$ $400 \mathrm{~N}$.

Segments bei zunehmendem axialem Kraftangriff deutlich ansteigt, kann auch hier ein Hinweis auf die innere Reibung in der Bandscheibe sein (Abb. 3.2.35). 


\section{$\underline{\text { Rotationssteifigkeit } \underline{B}_{\underline{G}}(\alpha)}$}

Die differentielle Steifigkeit nimmt leicht zu (Abb. 3.2.36). Die Applikation von hohen axialen Vorlasten erzeugt eine geringere anfängliche Rotationssteifigkeit des Segments (350 $\mathrm{Ncm} / \mathrm{deg}$ ) und eine Erhöhung am Ende eines Halbzykluses $\left(B_{G} \cong 270\right.$ $\mathrm{Ncm} /{ }^{\circ}$ ). Die minimale Steifigkeit vergrößert sich und beträgt $70 \mathrm{Ncm} /$.

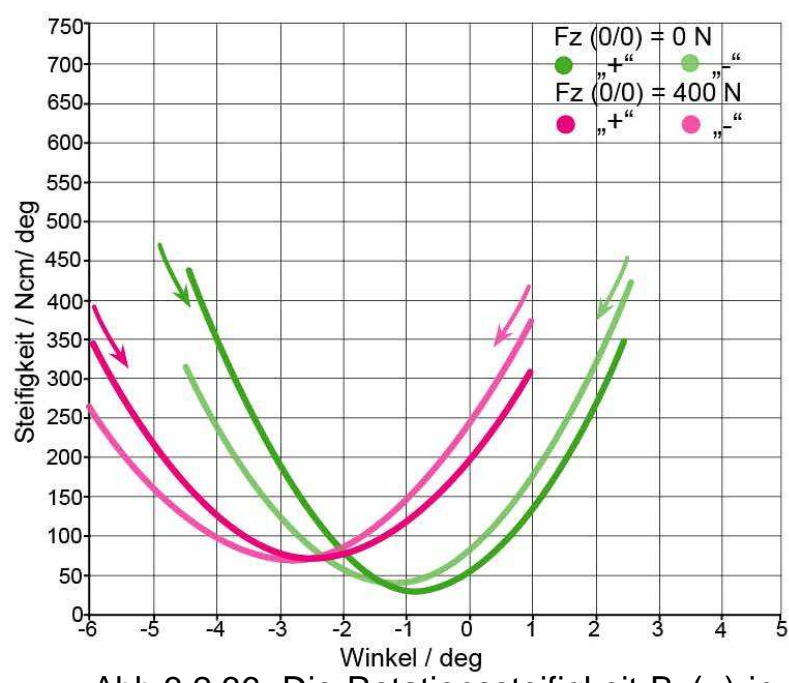

Abb.3.2.36: Die Rotationssteifigkeit $B_{G}(\alpha)$ in Abhängigkeit des Drehwinkels. Axialrotation in WZ: grün ohne Vorlast, pink mit $400 \mathrm{~N}$ Vorlast.

\section{$\underline{\text { Richtungsvektor der Schraubachse e ( } \alpha \text { ) }}$}

Der Wert der z-Komponente ist $e_{z}(\alpha)=1$ für Rechts- und Linksdrehung. Die anderen Komponenten sind den Komponenten eines Bewegungssegments ohne axiale Belastung sehr ähnlich. Nur für Drehwinkel $\alpha \geq 0,5^{\circ}$ sind alle Einheitsvektoren parallel zueinander, so dass die resultierenden Schraubachsen nur für diese Winkelbereiche parallel zum Drehmomentvektor liegen. In dem Fall steht der Richtungsvektor $\mathrm{e}(\alpha)$ senkrecht zur Bandscheibenebene. Bei der Richtungsumkehr wechselt das Vorzeichen des Richtungsvektors e $(\alpha)$ und auch das seiner Komponenten $e_{X}, e_{Y}$ und $e_{Z}$. Die Neigung der Schraubachse ist also in Vergleich zur $F_{Z}=0$ unverändert.

\section{Schraubsteigung $\tau(\alpha)$}

Abbildung 3.2.37 (S. 106) zeigt den Unterschied im Verlauf der momentanen Schraubsteigung $\tau=\tau(\alpha)$ für verschiedenen Vorlastgrößen. Die differentielle Schraubsteigung $\tau(\alpha)$ ist in der Einheit $\mathrm{mm} /$ Grad aufgetragen und über den 
Drehwinkel $\alpha$ präsentiert. Die Schraubsteigung $\tau(\alpha)$ für eine größere Vorlast von $400 \mathrm{~N}$ in Widerstandszenrum steigt um das 100-fache an. Die Bandscheibe wird sowohl für eine Links-, als auch für eine Rechtsdrehung um $s_{\max }=0,55 \mathrm{~mm}$ komprimiert.

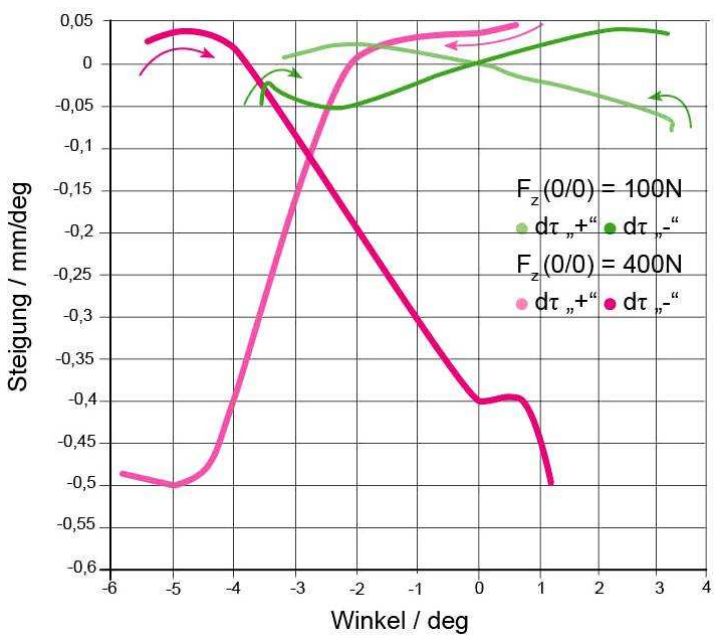

Abb.3.2.37: Die momentane Schraubsteigung $\tau(\alpha)$ in Abhängigkeit des Drehwinkels. Axialrotation in WZ: grün $100 \mathrm{~N}$ Vorlast, pink mit $400 \mathrm{~N}$ Vorlast.

Für jede Vorlastvariation wurden sowohl die Richtungskomponente der IHA wie auch die momentane Schraubsteigung bestimmt. Für folgende Messungen wird eine axiale Vorlast $F_{Z}=200 \mathrm{~N}$ gewählt, um den maximalen Effekt zu beschreiben und um beide Bewegungssegmente Th2/3 A und Th2/3 B nachfolgend vergleichen zu können.

\subsubsection{Verschiebungen entlang der $x$-Achse}

$$
I F_{Z} I=200 N ; \underline{I}(t)=\left(0,0, I_{Z}(t)\right), F\left(x_{F} / 0\right)=\left(0,0, F_{Z}\right)
$$

In der Abbildung 3.2.9 (S. 85) wird die Verschiebung der applizierten Vorlast $\mathrm{F}_{Z}$ $\left(\mathrm{x}_{\mathrm{F}} / 0\right)=200 \mathrm{~N}$ schematisch dargestellt. Die Vorlast wird systematisch in $5 \mathrm{~mm}$ Schritten entlang der $x$-Achse verschoben. Es kommt zu einer Flexion (Verkippung des oberen Wirbelkörpers nach ventral) und Extension (Verkippung des oberen Wirbels nach dorsal).

Das Widerstandszentrum $W_{Y}$ liegt in der Position $x_{F}=+5 \mathrm{~mm}$ für Th2/3 $B$, und befindet sich annähernd in der Mitte der Zwischenwirbelscheibe. 


\section{$\underline{\text { Rastpolkurven } r(\alpha)}$}

Bei dem Bewegungssegment Th2/3 B (Abb. 3.2.38 A) liegt $F_{Z}$ dorsal des ermittelten Widerstandszentrums $\left(\mathrm{X}_{\mathrm{F}}=-25 \mathrm{~mm}\right)$. Die Rastpolkurve zeigt einen wesentlichen Unterschied im Verlauf vergleichend $\mathrm{zu}$ der zentralen Vorlastapplikation (Abb.3.2.34, S.103).
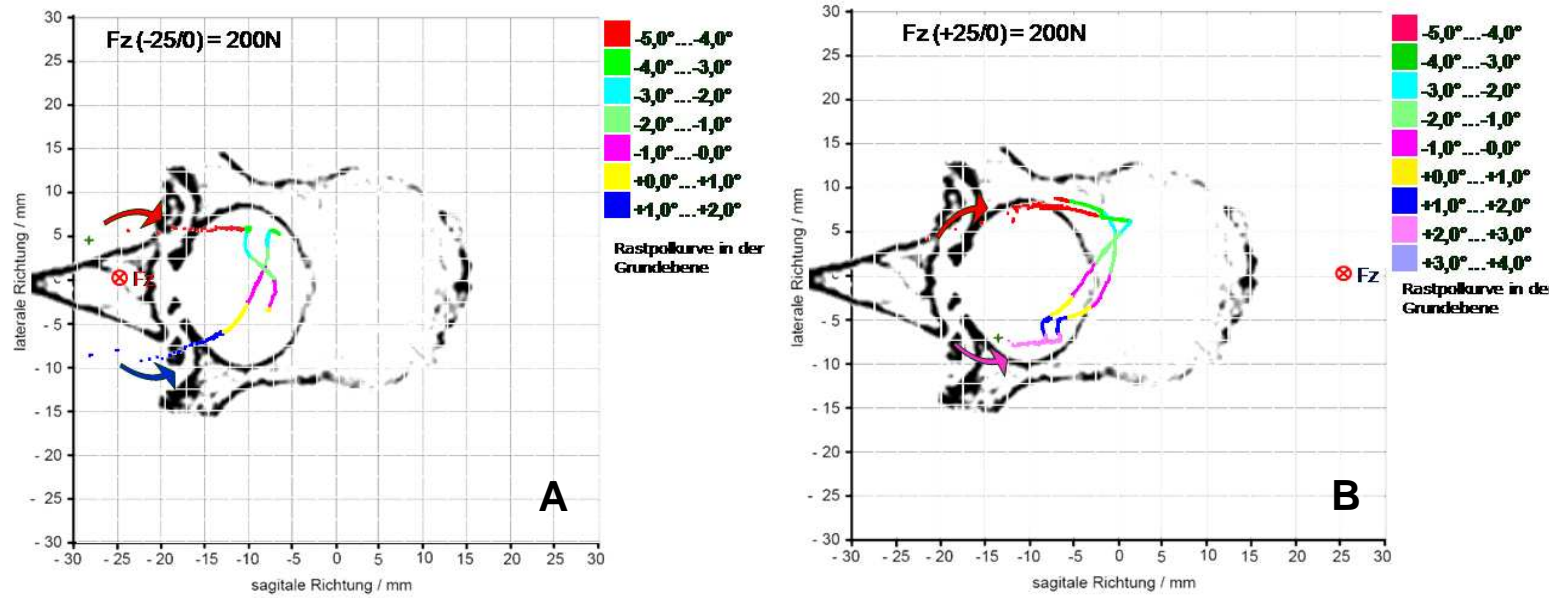

Abb. 3.2.38 A/B: Rastpolkurven von Th2/3 B.

Durchstoßpunkte $R_{i}(\alpha)$ der momentanen Schraubachsen bei dorsaler Vorlastposition $\left(\mathbf{X}_{\mathbf{i}}=-25 \mathrm{~mm}\right)(\mathbf{A})$, bei ventraler Vorlastposition $\left(\mathbf{X}_{\mathbf{i}}=+25 \mathrm{~mm}\right)(\mathbf{B})$.

Bei einer dorsalen Vorlast (Abb. 3.2.38 A) wandern die momentanen Schraubachsen zwischen den Wirbelbogengelenken entlang einer in Richtung der Bandscheibenmitte laufenden Rastpolkurve bis $\mathrm{x}_{\mathrm{i}}=-10 \mathrm{~mm}$, danach drückt sich die Rastpolkurve etwas nach dorsal. Die Durchstoßpunkte der momentanen Schraubachsen laufen zwischen den beiden Gelenken. Die Endpunkte der Rastpolkurve liegen in der Projektion des Wirbelkanals. Der genauere Verlauf wird folgend beschrieben: In Linksdrehung (gegen Uhrzeigersinn in der Aufsicht) wandert die Schraubachse von dorsal kommend nach ventral beginnend mit einer Verkippung $(\alpha=+29$. Sie läuft durch die Mitte des Wirbelkanals nach links lateral, macht einen kleinen Schlenker nach rechts-ventral und endet dort. Dann springt die momentane Schraubachse nach dorsal zur Gegenseite in den Bereich der linken Art. zygapophysialis, die Rechtsdrehung setzt ein. Im Laufe der Rechtsdrehung beschreibt die Rastpolkurve einen in der Linksdrehung ähnlichen Bogen. 
Die Rastpolkurven besitzen deutliche qualitative Unterschiede in Abhängigkeit der Vorlastposition $\mathrm{x}_{\mathrm{F}}$.

Bei der Positionierung von $F_{Z}$ ventral des Wirbelkanals $\left(x_{F}>0 \mathrm{~mm}\right)$ beschreibt die Rastpolkurve sowohl für Links- als auch für Rechtsdrehung ein nach dorsal offenen ungleichmäßig geformten ventralkonvexen Bogen (Abb. 3.2.38 B, S. 107). Der absolute Drehwinkel $\alpha$ als Maß der Rotationsbewegung ist sowohl bei Extension $\left(x_{F}<0\right)$, als auch bei Flexion $\left(x_{F}>0\right)$ gleichmäßig entlang der Rastpolkurve $r_{i}(\alpha)$ verteilt. Das sollte einer konstanten Wanderungsgeschwindigkeit der IHA entsprechen.

\section{Drehwinkel-Drehmoment-Kennlinien $\alpha(T)$}

Die Form der $\alpha(\mathrm{T})$ Funktion weist unabhängig von der Positionierung der Vorlast stets eine sigmoide Form auf (Abb. 3.2.39). Im Einzelnen zeigt sich eine deutliche Reduktion des Bewegungsumfangs für dorsale Vorlastpositionen $\left(\mathrm{x}_{\mathrm{F}}<0\right)$ von $|\alpha|=7^{\circ}$ (für zentrale Vorlast) auf $|\alpha|=5,4^{\circ}$, was einer Abnahme des ROMs von $22,8 \%$ entspricht. Die Zunahme ROMs für ventrale $\left(x_{F}>0\right)$ Vorlastpositionen auf $|\alpha|=7,6^{\circ}$ ist gering und entspricht nur $8,5 \%$.

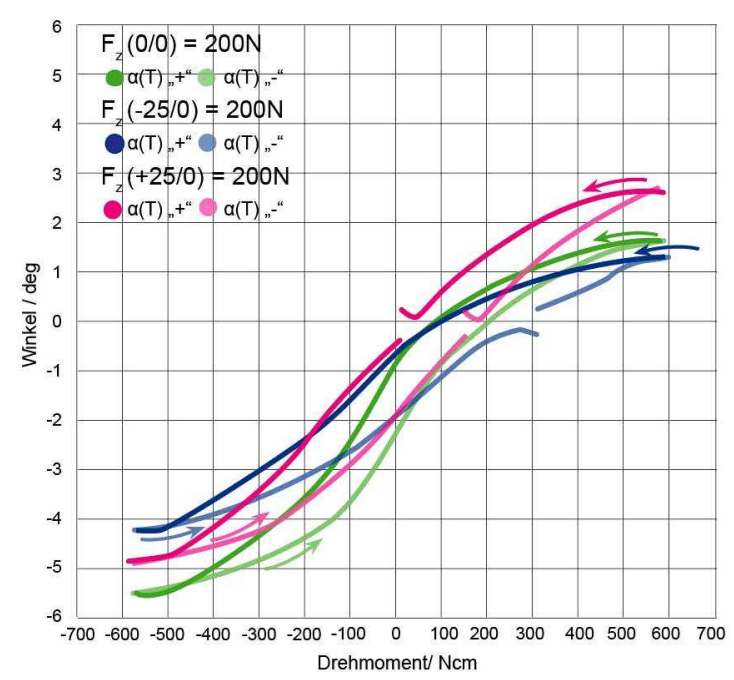

Abb. 3.2.39: Drehwinkel-DrehmomentKennlinien. Axialrotation 200N in WZ: in grün $F_{Z}(0 / 0)$, pink $F_{Z}(+25 / 0)$, blau $F_{Z}(-25 / 0)$. 


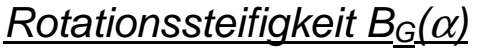

Die differentielle Steifigkeit bei Applikation einer axialen Vorlast ventral oder dorsal des WZ unterscheidet sich unwesentlich von der im Widerstandszentrum (Abb. 3.2.40). Die minimale Steifigkeit bei ventralen Vorlasten im Vergleich zu zentralen Vorlasten bleibt annähend gleich. Bei dorsalen Vorlasten ist die minimale Rotationssteifigkeit etwas höher und liegt bei $B_{G} \cong 125 \mathrm{Ncm} /{ }^{\circ}$.

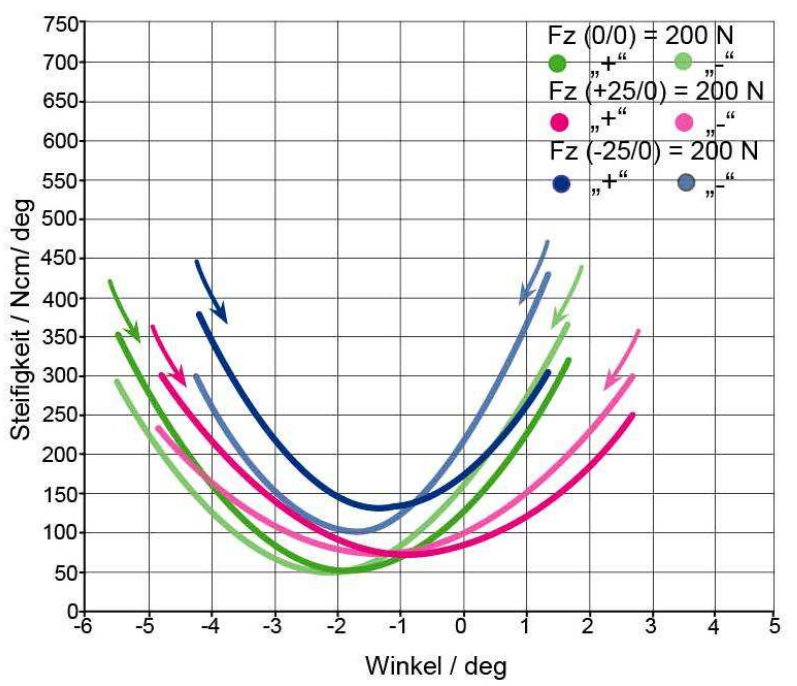

Abb.3.2.40: Die Rotationssteifigkeit $\mathrm{B}_{\mathrm{G}}(\alpha)$ in Abhängigkeit des Drehwinkels. Axialrotation $200 \mathrm{~N}$ Vorlast: in grün $F_{Z}(0 / 0)$, pink $F_{Z}(+25 / 0)$, blau $F_{Z}$ $(-25 / 0)$.

\section{Richtung der momentanen Schraubachsen e (r)}

Eine Verlagerung des Kraftvektors nach dorsal ändert das Verhalten des Richtungsvektors der $\mathrm{IHA}\left(\mathrm{T}_{\mathrm{z}}\right)$. Die Rastpolkurven stehen im negativen Winkelbereich $\alpha$ von $-2^{\circ}$ bis $-1,5$ parallel zueinander und zum applizierten Drehmoment $T_{Z}(t)$. In der Darstellung der einzelnen Komponenten des Richtungsvektors $\underline{e}$ für eine dorsale Vorlastposition steigt die ey-Komponente an (Abb. 3.2.41, S. 110). Bei einer Linksdrehung (positive Rotationsrichtung) schwenkt die momentane Schraubachse in der Frontalebene von rechts nach links und für Rechtsdrehung umgekehrt von links nach rechts. Der Neigungswinkel beträgt $81,96^{\circ}$ nach links-lateral. Somit verkippt sich die momentane Schraubachse um $8,04^{\circ}$ von der senkrechten Stellung nach links. Die IHA verkippt während der Gesamtrotation nach ventral. In Abbildung 3.2.42 (S. 110) wird das Verhalten des Richtungsvektors $\mathrm{e}(\alpha)$ und seiner Komponenten $e_{X}, e_{Y}$ und $e_{Z}$ bei Applikation einer ventralen Vorlast verdeutlicht.

Die Schwankung der IHA bei einer ventralen Belastung ist erheblich. Die Neigung der Schraubachse wechselt im Laufe der Bewegung mehrmals. Die Schraubachse 
schwenkt in der Frontalebene bei der Rechtsdrehung von rechts nach links um 5,2 $2^{\circ}$ und bei der Linksdrehung von links nach rec hts um 10,8 . Die Verkippung in der Sagittalebene ist drehwinkelabhängig.

Die $e_{X}$-Komponente hat bei einer Rechtsdrehung über den Rotationsbereich $\alpha \geq-1,5^{\circ}$ einen positiven Wert und bei $\alpha \leq-1,5^{\circ}$ einen negativen Wert. Für Linksdrehung ist ihr Verhalten umgekehrt: einen positiven Wert für negative Drehwinkel $\alpha \leq-1,5^{\circ}$ und einen negativen Wert für $\alpha \geq-1,5^{\circ}$. Das entspricht einer Verkippung der IHA während der Rechtsdrehung erst nach ventral, dann richtet sich die Schraubachse senkrecht zur Bandscheibenebene auf, um dann nach dorsal zu verkippen. Die Linksdrehung setzt ein. Zu Beginn der Linksdrehung ist die Schraubachse nach dorsal geneigt, wobei der Neigungswinkel zur Sagittalebene sich mit ansteigendem Drehwinkel weiter verkleinert. Die Linksdrehung endet mit einer nach ventral gekippten Schraubachse.

Der maximale Neigungswinkel liegt zwischen $17,5^{\circ}$ na ch ventral für $\alpha \leq-1,5^{\circ}$ und $30,2^{\circ}$ nach dorsal für den Drehwinkelbereich $\alpha \geq-1,5^{\circ}$ (Abb. 3.4.42).

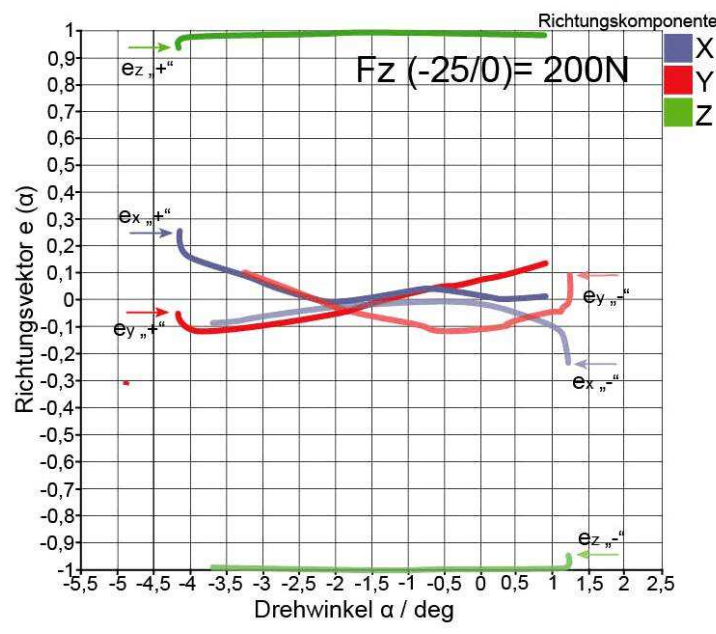

Abb. 3.2.41: Richtungsvektors $\underline{e}$ der momentanen Schraubachsen in Abhängigkeit von $\alpha$ für eine dorsale Vorlastposition $F_{Z}(-25 / 0)$.

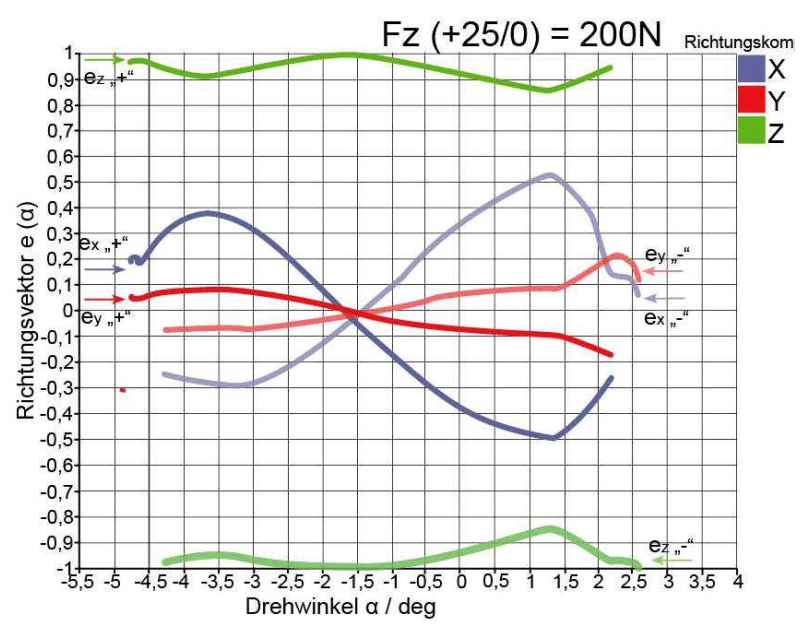

Abb. 3.2.42: Richtungsvektors $\underline{e}$ der momentanen Schraubachsen in Abhängigkeit von $\alpha$ für eine ventrale Vorlastposition $\mathrm{F}_{Z}(+25 / 0)$. 


\section{Schraubsteigung}

Für die Drehwinkel $\alpha$ in der Rechtsdrehung für $\mathrm{F}_{Z}(0 / 0)$ bei konstanter Vorlast von $200 \mathrm{~N}$ ist die Abhängigkeit näherungsweise linear. Mit steigendem Drehwinkel nimmt die Schraubsteigung zu. Bei einer Linksdrehung ist die Abhängigkeit umgekehrt proportional der Rechtsdrehung. Für Hin- und Rücklauf innerhalb eines Messzyklus ist für $\alpha=1,8^{\circ}$ die Schraubsteigung gleich Null. Die Band-

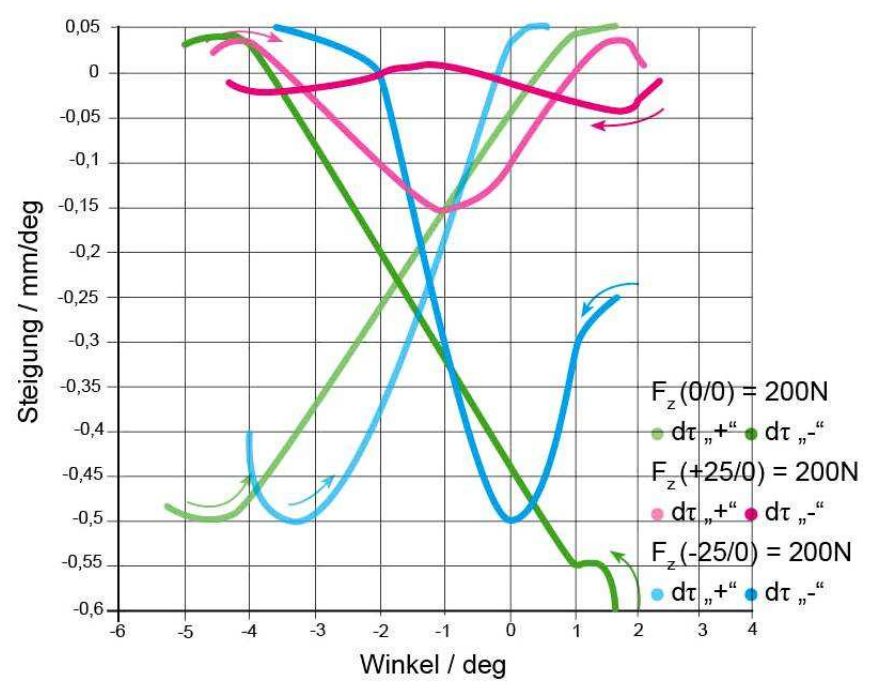

Abb. 3.2.43: Schraubsteigung $\tau(\alpha)$ der momentanen Schraubachsen von Th2/3 B bei verschiedenen Vorlastpositionen.

scheibe wird sowohl für eine Links-, als auch für eine Rechtsdrehung um $S_{\max }=0,64 \mathrm{~mm}$ elongiert. Die berechneten Werte für $F_{Z}(0 / 0)$ liegen zwischen $-0,6$ $\mathrm{mm} /$ Grad für Kompression und 0,04 mm/ Grad für Elongation der Bandscheibe (Abb. 3.2.43).

Auch bei dem zweiten Bewegungssegment gibt es große Unterschiede zwischen der Schraubsteigung bei den ventralen und dorsalen Vorlastpositionen. Die Schraubsteigung für dorsale Vorlast mit Rotationswinkelbereich von $|\alpha|=5^{\circ}$ zeigt eine Steigung $\tau(\alpha)$ von $-0,5 \mathrm{~mm} / \mathrm{Grad}$ bis $0,05 \mathrm{~mm} /$ Grad. Für ventrale Vorlast ist der maximale Rotationsbereich größer und liegt bei $7^{\circ}$. Zu Beginn der Linksdrehung kommt es zur einen Abwärtsbewegung des Segments bis $\alpha \approx-1^{\circ}$, wo es in eine Aufwärtsbewegung des oberen Wirbels umschlägt (positive Drehwinkel). Bei der Rechtsdrehung ist diese Abfolge umgekehrt. Die maximale Schraubsteigung liegt zwischen $-0,15 \mathrm{~mm} /$ Grad und 0,01 mm/ Grad. 


\subsubsection{Verschiebung der axialen Vorlast entlang der y-Achse;}

$$
\mathrm{FZ}\left(\mathrm{x}_{\mathrm{F}} / \mathrm{y}_{\mathrm{F}}\right) ; \mathrm{IF}_{\mathrm{Z}} \mathrm{I}=\mathbf{2 0 0 \mathrm { N }} ; \underline{\mathrm{I}}(\mathrm{t})=\left(0,0, \underline{I}_{\mathrm{Z}}(\mathrm{t})\right)
$$

Die Vorlast $F_{Z}=200 \mathrm{~N}$ wird entlang der $y$-Reiter ausgehend vom Widerstandszentrum $W_{Y}(0 / 0)$ nach rechts und links verschoben (3.2.26 A, B, S. 97). Es resultiert eine laterale Verkippung des oberen Wirbelkörpers nach links (bei $y_{F}>0$ ) bzw. nach rechts $\left(\right.$ bei $\left.y_{F}<0\right)$.

\section{$\underline{\text { Rastpolkurven } r_{i}(\alpha)}$}

Das Verhalten der momentanen Schraubachsen für Vorlastpositionen $-10 \mathrm{~mm} \leq \mathrm{y}_{\mathrm{F}}$ $<10 \mathrm{~mm}$ ändert sich kaum. Zu einer qualitativen Veränderung kommt es erst bei einer Verschiebung von $\mathrm{F}_{Z}$ um mehr als $20 \mathrm{~mm}$.

Bei dem zweiten Präparat, nach Ansetzen einer lateralen Kraft, werden ähnliche, nach dorsal offene bogenförmige Kurven gemessen. Gleichzeitig sind die Rastpolkurven bei der lateralen Vorlast in ihrer Ausbreitung komprimierter als die bei der dorsalen Vorlastapplikation (Abb. 3.2.44 A/ B, S. 113).

Es wird zuerst die Abbildung 3.2.44 A analysiert. Die Rastpolkurven verbleiben in der Bandscheibenmitte, anstatt sich zur Seite der jeweiligen Belastung zu verlagern, wie es bei dem Bewegungssegment Th 2/3 A der Fall war. Desweiteren wird ein deutlicher Unterschied zwischen einer Rechts- und einer Linksdrehung des oberen Segments innerhalb eines Messzyklus registriert.

Die Rastpolkurve verläuft bei der rechtsseitigen Belastung ausgehend vom rechten Gelenk in die Bandscheibe. Der Rotationsumfang $\alpha(T)$ ist in positive Richtung der Axialrotation mit $\alpha(T)=5^{\circ} \rightarrow-1^{\circ}$ reduziert.

Nach dem Entfernen aus der Rechtsposition bleibt die Rastpolkurve rechts-ventral liegen. Die Kurve zeigt einen verkürzten und komprimierten Verlauf. Bei maximaler Drehung nach rechts zur Seite der Belastung endet die IHA mittig in dem Wirbelkanal. Der Rotationsumfang $\alpha(T)$ ist in negative Richtung der Axialrotation mit $\alpha(\mathrm{T})=-2^{\circ} \rightarrow 4^{\circ}$ reduziert (Abb. 3.2.44 A). 
Bei der linksseitigen Belastung des zweiten Segments ist eine geringfügige Verlagerung der gesamten IHA nach links-dorsal feststellbar. Es ist eine kleinere ventral leicht komprimierte Konvexität der Rastpolkurve erkennbar.

Auch bei Lateralflexion um $\kappa_{x}<0$ durch axiale Kraft mit Ansatz am $y_{F}>20 m m$ kommt es zu einer Reduktion des Rotationsumfangs $\alpha(T)$ in negative Richtung mit $\alpha(T)=-2^{\circ} \rightarrow 4^{\circ}($ Abb. 3.2.44 B).
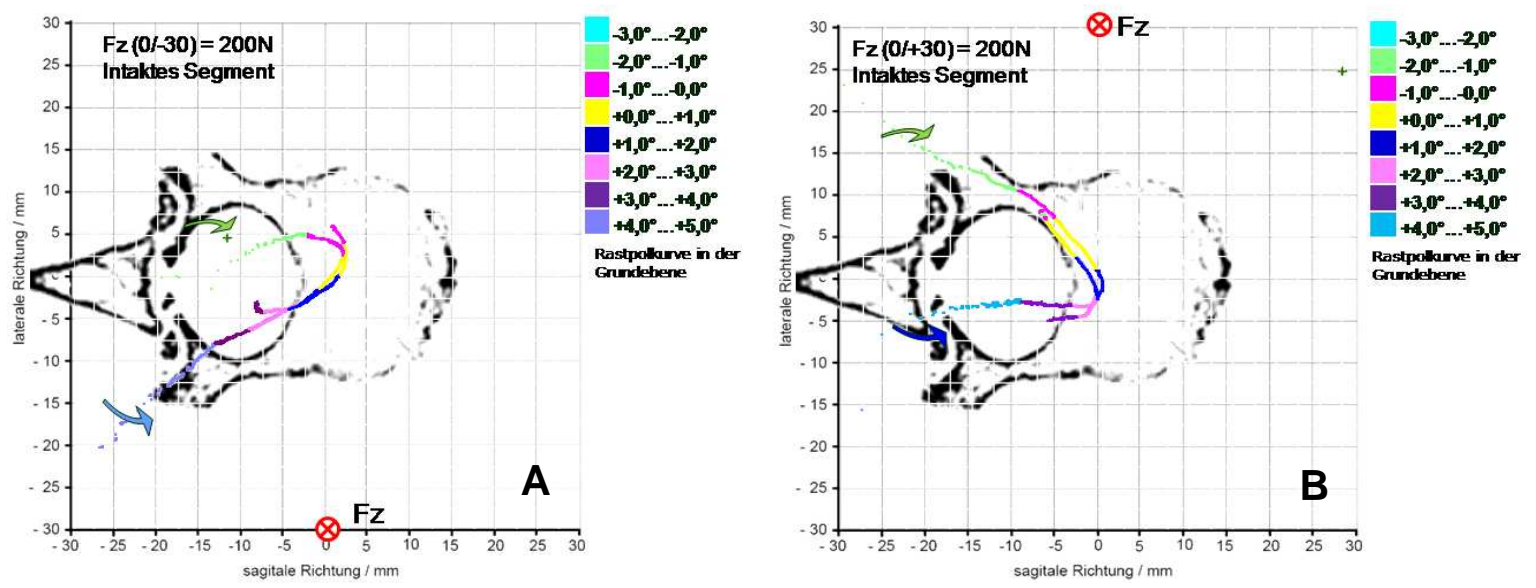

Abb. 3.2.44 A/B: Rastpolkurven von Th2/3 B. Durchstoßpunkte $R_{i}(\alpha)$ der momentanen Schraubachsen bei Vorlastposition in $\mathrm{y}_{\mathrm{F}}=-30 \mathrm{~mm}(\mathbf{A})$ und in $\mathrm{y}_{\mathrm{F}}=+30 \mathrm{~mm}(\mathbf{B})$. Die gebogenen Pfeile geben die Laufrichtung der Rastpolkurve und die Rotationsrichtung des oberen Wirbels an.

Die Steifigkeit bei der axialen Rotation nimmt bei vorhergegangener lateraler Verkippung des oberen Wirbelkörpers ab. Bei Verlagerung der Vorlast nach rechts oder nach links wird keine nennenswerte Änderung des ROMs sowie der Hysterese $\Delta \alpha(T=0)$ gemessen. Die Richtungskomponente der Schraubachsen, insbesondere die größere y-Komponente des Richtungsvektors $\mathrm{e}(\alpha)$, und die momentane Schraubsteigung $\tau(\alpha)$ korreliert mit dem einseitigen Anstieg der Steifigkeit. Der Verlauf der momentanen Schraubsteigung $\tau(\alpha)$ zeigt ein lineares Verhalten. 


\subsubsection{Vergleich intakter Bewegungssegmente Th 2/3 A und Th 2/3 B}

\begin{tabular}{|c|c|c|}
\hline Merkmal & intaktes Segment Th 2/3 A & intaktes Segment Th 2/3 B \\
\hline \multicolumn{3}{|c|}{ Verhalten der IHAs bei Änderung der Vorlastposition } \\
\hline dorsale Vorlast & $\begin{array}{l}\text { IHAs mittig in der Bandscheibe, geringe } \\
\text { IHA-Wanderung. } \\
\text { max. Rechtsdrehung: } \\
\text { Verkippen nach rechts und ventral. } \\
\text { max. Linksdrehung: } \\
\text { Verkippen nach links und ventral. } \\
\end{array}$ & $\begin{array}{l}\text { IHAs nach dorsal verlagert, gerin- } \\
\text { ge IHA-Wanderung. } \\
\text { max. Rechtsdrehung: } \\
\text { Verkippen nach rechts und ventral } \\
\text { max. Linksdrehung: } \\
\text { Verkippen nach links und ventral. } \\
\end{array}$ \\
\hline ventrale Vorlast & $\begin{array}{l}\text { IHAs verlaufen in weit nach dorsal } \\
\text { offenem Bogen, gegenläufig zur } \\
\text { Rotationsrichtung. } \\
\text { max. Rechtsdrehung: } \\
\text { Verkippen nach links und dorsal. } \\
\text { max. Linksdrehung: } \\
\text { Verkippen nach rechts und dorsal. }\end{array}$ & $\begin{array}{l}\text { IHAs verlaufen in weit nach dorsal } \\
\text { offenem Bogen, gegenläufig zur } \\
\text { Rotationsrichtung. } \\
\text { max. Rechtsdrehung: } \\
\text { Verkippen nach links. } \\
\text { max. Linksdrehung: } \\
\text { Verkippen nach rechts. } \\
\text { In der Sagittalebene ist die Ver- } \\
\text { kippung drehwinkelabhängig. }\end{array}$ \\
\hline laterale Vorlast & $\begin{array}{l}\text { IHAs deutlich zur Seite der Belastung } \\
\text { Verschoben. } \\
\text { max. Rechtsdrehung: } \\
\text { Verkippen nach rechts und dorsal. } \\
\text { max. Linksdrehung: } \\
\text { Verkippen nach links und ventral. }\end{array}$ & $\begin{array}{l}\text { IHAs nur leicht zur Seite der Be- } \\
\text { lastung verschoben. } \\
\text { max. Rechtsdrehung: } \\
\text { Verkippen nach rechts und dorsal. } \\
\text { max. Linksdrehung: } \\
\text { Verkippen nach links und ventral. }\end{array}$ \\
\hline \multicolumn{3}{|c|}{ Verhalten von ROM und Steifigkeit } \\
\hline dorsale Vorlast & $\begin{array}{l}\text { ROM } \downarrow \\
\text { Hysterese } \downarrow \\
\text { minimale Steifigkeit } \uparrow\end{array}$ & $\begin{array}{l}\text { ROM } \downarrow \\
\text { Hysterese } \downarrow \\
\text { minimale Steifigkeit } \uparrow\end{array}$ \\
\hline ventrale Vorlast & $\begin{array}{l}\text { ROM } \downarrow \\
\text { Hysterese } \downarrow \\
\text { minimale Steifigkeit } \uparrow \text { geringfügig }\end{array}$ & $\begin{array}{l}\mathrm{ROM} \uparrow \\
\text { Hysterese } \uparrow \\
\text { minimale Steifigkeit } \uparrow \text { geringfügig }\end{array}$ \\
\hline laterale Vorlast & $\begin{array}{l}\text { ROM unverändert } \\
\text { minimale Steifigkeit minimal } \uparrow\end{array}$ & $\begin{array}{l}\text { ROM unverändert } \\
\text { minimale Steifigkeit minimal } \uparrow\end{array}$ \\
\hline
\end{tabular}

Tab.3.1: Zusammenfassung der Ergebnisse der Versuchsreihe „Axialrotation“ für Th2/3 A und Th2/3 B. 


\subsubsection{Vergleich intaktes Bewegungssegment und Segment nach der Resektion der rechten Art. zygapophysialis (Th 2/3 A)}

\begin{tabular}{|c|c|c|}
\hline Merkmal & intaktes Segment Th $2 / 3 \mathrm{~A}$ & $\begin{array}{l}\text { Resektion rechten Art. zygapo- } \\
\text { physialis }\end{array}$ \\
\hline \multicolumn{3}{|c|}{ Verhalten der IHAs bei Änderung der Vorlastposition } \\
\hline dorsale Vorlast & $\begin{array}{l}\text { IHAs mittig in der Bandscheibe, } \\
\text { komprimiert. } \\
\text { max. Rechtsdrehung: } \\
\text { Verkippen nach rechts und ventral. } \\
\text { max. Linksdrehung: } \\
\text { Verkippen nach links und ventral. }\end{array}$ & $\begin{array}{l}\text { IHAs stärker komprimiert. } \\
\text { Position gleich. } \\
\text { Ausrichtung der IHA wie bei zent- } \\
\text { raler Vorlastposition. }\end{array}$ \\
\hline ventrale Vorlast & $\begin{array}{l}\text { IHAs verlaufen in weit nach dorsal } \\
\text { offenem Bogen, gegenläufig zur } \\
\text { Rotationsrichtung. } \\
\text { max. Rechtsdrehung: } \\
\text { Verkippen nach links und dorsal. } \\
\text { max. Linksdrehung: } \\
\text { Verkippen nach rechts und dorsal. } \\
\end{array}$ & $\begin{array}{l}\text { Verlauf in schmalen nach dorsal } \\
\text { offenem Bogen, gegenläufig zur } \\
\text { Rotationsrichtung. } \\
\text { max. Rechtsdrehung: } \\
\text { Verkippen nach links und dorsal. } \\
\text { max. Linksdrehung: } \\
\text { Verkippen nur nach dorsal. } \\
\end{array}$ \\
\hline laterale Vorlast & $\begin{array}{l}\text { IHAs deutlich zur Seite der Belastung } \\
\text { verschoben. } \\
\text { max. Rechtsdrehung: } \\
\text { Verkippen nach rechts und dorsal. } \\
\text { max. Linksdrehung: } \\
\text { Verkippen nach links und ventral. }\end{array}$ & $\begin{array}{l}\text { IHAs nur zur Seite des vorhande- } \\
\text { nen Gelenks verschoben. } \\
\text { max. Rechtsdrehung: } \\
\text { Verkippen nach rechts und dorsal. } \\
\text { max. Linksdrehung: } \\
\text { Verkippen nach ventral. }\end{array}$ \\
\hline \multicolumn{3}{|c|}{ Verhalten von ROM und Steifigkeit } \\
\hline dorsale Vorlast & $\begin{array}{l}\text { ROM } \downarrow \\
\text { Hysterese } \downarrow \\
\text { minimale Steifigkeit } \uparrow \\
\end{array}$ & $\begin{array}{l}\text { ROM unverändert } \\
\text { Hysterese unverändert } \\
\text { minimale Steifigkeit unverändert } \\
\end{array}$ \\
\hline ventrale Vorlast & $\begin{array}{l}\text { ROM } \downarrow \\
\text { Hysterese } \downarrow \\
\text { minimale Steifigkeit } \uparrow \text { geringfügig }\end{array}$ & $\begin{array}{l}\text { ROM } \uparrow \\
\text { Hysterese } \uparrow \\
\text { minimale Steifigkeit unverändert }\end{array}$ \\
\hline laterale Vorlast & $\begin{array}{l}\mathrm{ROM} \uparrow \\
\text { minimale Steifigkeit } \uparrow\end{array}$ & $\begin{array}{l}\mathrm{ROM} \uparrow \\
\text { minimale Steifigkeit unverändert. }\end{array}$ \\
\hline
\end{tabular}

Tab.3.2: Zusammenfassung der Ergebnisse der Versuchsreihe „Axialrotation“ für Th 2/3 A nach

Resektion der rechten Art. zygapophysialis. 


\subsubsection{Vergleich intaktes Bewegungssegment und}

Bandscheibe- Bänder- Präparat (Th 2/3 A)

\begin{tabular}{|c|c|c|}
\hline Merkmal & intaktes Segment Th 2/3 A & Bandscheibe-Bänder-Präparat \\
\hline \multicolumn{3}{|c|}{ Verhalten der IHAs bei Änderung der Vorlastposition } \\
\hline dorsale Vorlast & $\begin{array}{l}\text { IHAs mittig in der Bandscheibe, } \\
\text { geringe IHA-Wanderung. } \\
\text { max. Rechtsdrehung: } \\
\text { Verkippen nach rechts und ventral. } \\
\text { max. Linksdrehung: } \\
\text { Verkippen nach links und ventral. }\end{array}$ & $\begin{array}{l}\text { IHAs Verlauf von dorsal kom- } \\
\text { mend, mittig geradeaus in die } \\
\text { Bandscheibe. } \\
\text { parallel zu Tz. }\end{array}$ \\
\hline ventrale Vorlast & $\begin{array}{l}\text { IHAs verlaufen in weit nach dorsal } \\
\text { offenem Bogen, gegenläufig zur } \\
\text { Rotationsrichtung. } \\
\text { max. Rechtsdrehung: } \\
\text { Verkippen nach links und dorsal. } \\
\text { max. Linksdrehung: } \\
\text { Verkippen nach rechts und dorsal. }\end{array}$ & $\begin{array}{l}\text { IHAs liegt zentral und ortsfest im } \\
\text { vorderen Drittel der Bandscheibe. } \\
\text { parallel zu Tz. }\end{array}$ \\
\hline laterale Vorlast & $\begin{array}{l}\text { IHAs deutlich zur Seite der Belastung } \\
\text { verschoben. } \\
\text { max. Rechtsdrehung: } \\
\text { Verkippen nach rechts und dorsal. } \\
\text { max. Linksdrehung: } \\
\text { Verkippen nach links und ventral. }\end{array}$ & $\begin{array}{l}\text { IHAs stets minimal zur Seite der } \\
\text { Belastung verschoben } \\
\text { parallel zu Tz }\end{array}$ \\
\hline \multicolumn{3}{|c|}{ Verhalten von ROM und Steifigkeit } \\
\hline dorsale Vorlast & $\begin{array}{l}\text { ROM } \downarrow \\
\text { Hysterese } \downarrow \\
\text { minimale Steifigkeit } \uparrow\end{array}$ & $\begin{array}{l}\mathrm{ROM} \uparrow \\
\text { Hysterese } \uparrow \\
\text { minimale Steifigkeit unverändert }\end{array}$ \\
\hline ventrale Vorlast & $\begin{array}{l}\text { ROM } \downarrow \\
\text { Hysterese } \downarrow \\
\text { minimale Steifigkeit } \uparrow \text { geringfügig }\end{array}$ & $\begin{array}{l}\mathrm{ROM} \uparrow \\
\text { Hysterese } \uparrow \\
\text { minimale Steifigkeit unverändert }\end{array}$ \\
\hline laterale Vorlast & $\begin{array}{l}\mathrm{ROM} \uparrow \\
\text { minimale Steifigkeit } \uparrow\end{array}$ & $\begin{array}{l}\mathrm{ROM} \uparrow \\
\text { minimale Steifigkeit unverändert }\end{array}$ \\
\hline
\end{tabular}

Tab.3.3: Zusammenfassung der Ergebnisse der Versuchsreihe „Axialrotation“ für Th 2/3 A nach

Resektion beider Artt. zygapophysiales. 


\subsection{Flexion und Extension $I(t)=\left(0, I_{Y}(t), 0\right)$}

Im Experiment „Flexion und Extension“ wird ein zyklisches, sich linear veränderndes Drehmoment $\underline{T}(\mathrm{t})=\left(0, \underline{I}_{Y}(\mathrm{t}), 0\right)$ aufgebracht. Die Abbildung 2.5.3 B (S. 59) zeigt die Aufbau des Messstands. Allerdings muss das Drehmoment $T_{Y}$ für beide Bewegungssegmente auf $400 \mathrm{Ncm}$ reduziert werden, um die Segmente nicht zu schädigen und alle Vorlastpositionen aufzeichnen zu können. Für jedes Segment wird erst ein Drehmoment $T_{Y}$ ohne axiale Vorlast appliziert und die resultierende Bewegung aufgezeichnet. Für alle weitere Messungen wird eine axiale Vorlast von $\mathrm{F}_{Z}=200 \mathrm{~N}$ gewählt. Der Ort ihrer Kraftwirkungslinie wird entlang der $\mathrm{x}$ und y-Achse variiert. Anschließend erfolgt die sukzessive Resektion des rechten und des linken Wirbelbogengelenks, wobei diese Untersuchung nur auf Segment Th 2/3 A beschränkt ist.

\subsubsection{Bewegungssegment Th 2/3 A}

\subsubsection{Applikation eines Drehmoments ohne axiale Vorlast;}

$$
\underline{I}(t)=\left(0, \underline{T}_{Y}(t), 0\right) ; F_{Z}=0
$$

\section{Rastpolkurven}

Die momentanen Schraubachsen werden als Durchstoßpunkt $R_{i}$ durch die Medianebene des Bewegungssegments dargestellt. Die Rastpolkurve $r_{i}(\alpha)$ verläuft von dorsal nach ventral in einem Bogen, der mittig und circa 18mm kaudal der Bandscheibe des Th2/3-Segments und somit knapp oberhalb der Bandscheibe des vierten thorakalen Wirbels liegt (Abb. 3.3.1, S. 118).

In maximaler Flexion (Drehwinkel von 1을 29 liegen die Rastpolkurven auf der Höhe der Bandscheibenmitte. Mit zunehmender Extension kommt es zu einer geringradigen Verlagerung der $\mathrm{IHA}\left(\mathrm{T}_{\mathrm{Y}}\right)$ um weitere $5 \mathrm{~mm}$ nach kaudal in die Bandscheibe des Junghannsschen Segments Th 3/4. Für Extensionswinkel $\alpha=0^{\circ}$ $\rightarrow-3^{\circ}$ verläuft die Rastpolkurve nach dorsal und ende t auf der gleichen Segmenthöhe wie zu Beginn der Extension. Bei Bewegungsumkehr springt die Rastpolkurve nach dorsal. 
Aus maximaler Extension heraus und von dorsal kommend, läuft sie nach ventral, macht mittig einen Schlenker nach kranial und endet in maximaler Flexionsstellung.

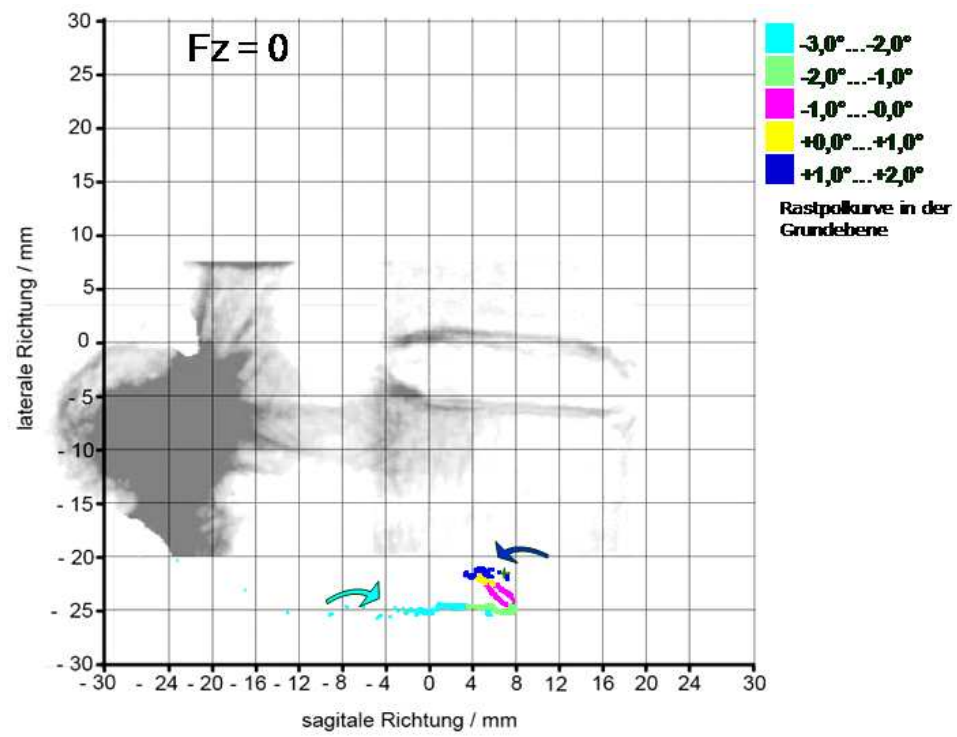

Abb. 3.3.1 (Th2/3 A): Ansicht von rechts.

Durchstoßpunkte $R_{i}(\alpha)$ der momentanen Schraubachsen für eine Drehung in positive (hellblau) und negative (dunkelblau) Richtung $\left(\mathbf{F}_{\mathbf{Z}}=\mathbf{0}\right)$. Die gebogenen Pfeile geben die Laufrichtung der Rastpolkurve und die Rotationsrichtung des oberen Wirbels an.

Drehwinkel- Drehmoment- Kennlinie

Die Drehwinkel-Drehmoment-Kennlinien haben eine deutlich sigmoide Form und sind für den Hin- und Rücklauf einer Messung nicht identisch. Sie zeigen eine messbare Hysterese $\Delta \alpha(T=0)$. Für $T=0$ war die Hysterese $\alpha=0,5^{\circ}$. Die $\alpha(T)$-Funktion ist sym-metrisch bezüglich der $y$ Achse, das heißt, dass die Bewegung eben und gleichmäßig verläuft. Der Bewegungsumfang (ROM) beträgt $4^{\circ}$ mit maximal angreifendem Drehmoment von $400 \mathrm{~N} / \mathrm{cm}$ (Abb.

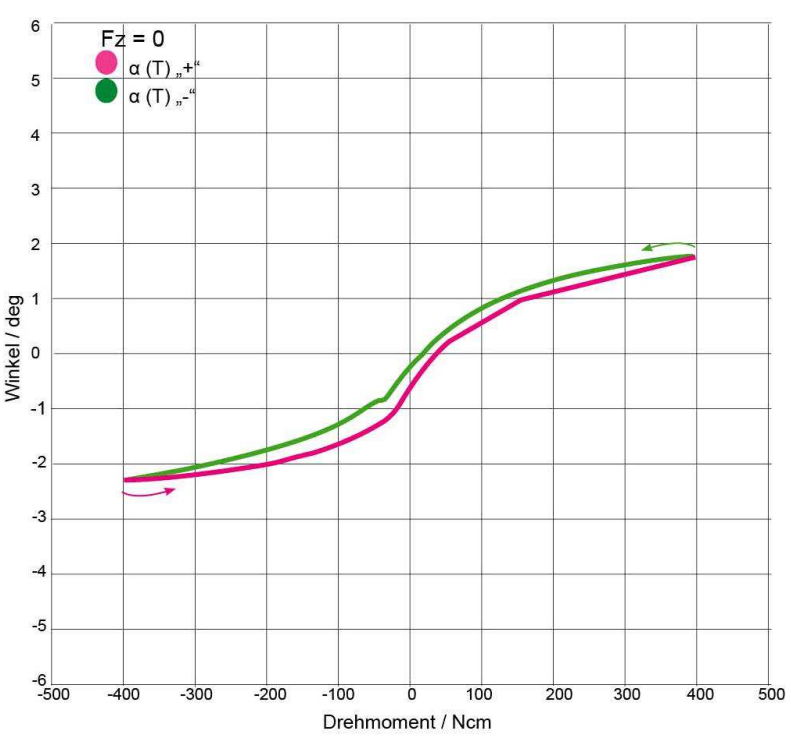

Abb.3.3.2: Drehmoment-Drehwinkel-Kennlinie. Die Pfeile zeigen die Laufrichtung an. Flexion positive und Extension negative Richtung.

3.3.2). 


\section{$\underline{\text { Rotationssteifigkeit } B_{G}(\alpha)}$}

Die Abbildung 3.3.3 zeigt die Steifigkeits-Drehwinkel-Funktion mit einer minimalen Steifigkeit $B(\alpha)=25-60 \%$ Ncm. Im Flexionsbereich, sowie im Extensionsbereich (negative Drehwinkel $\alpha$ ), steigt die Steifigkeit $B(\alpha)$ bis zu einem Maximum von $500^{\circ} / \mathrm{Ncm}$ an.

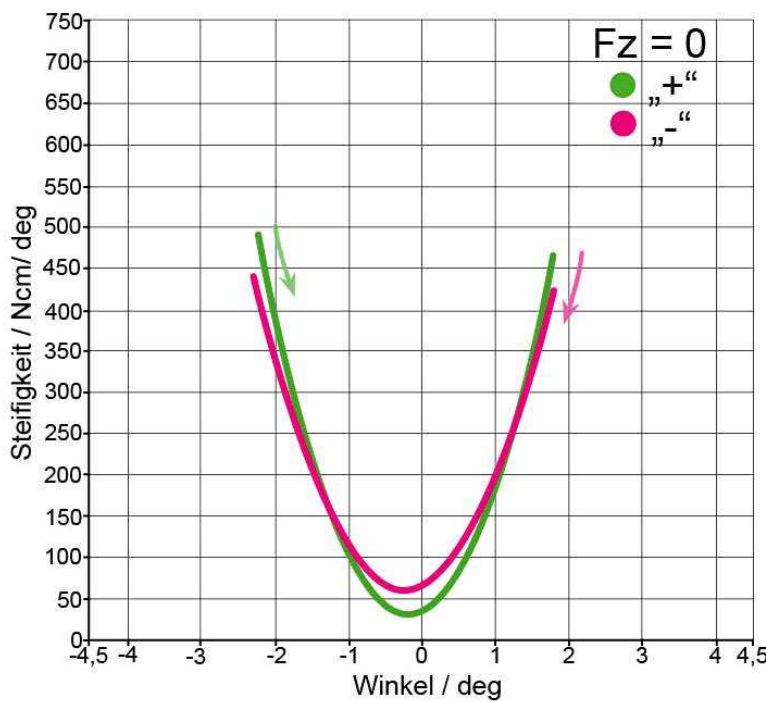

Abb.3.3.3: Die Rotationssteifigkeit $B_{G}(\alpha)$ in Abhängigkeit des Drehwinkels. Der erste Halbzyklus: grün (Flexion $(,+,$,$) ) und der$ zweite Halbzyklus pink (Extension (,,-“)).

Richtungsvektor der Schraubachse e $(\alpha)$ Die y-Komponente ist sowohl in Flexion- als auch für Extensionslage $e_{Y}(\alpha) \cong 1$, somit sind die IHAs parallel zum Drehmoment $T_{Y}$ ausgerichtet. Für Winkel $\alpha>0^{\circ}$ liegen die Schraubachsen in der Horizontalebene $e_{z}(\alpha) \cong 0$, und sind in maximaler Flexionslage leicht um $0,6^{\circ}$ gegen die Transversalebene nach dorsal gekippt. Mit zunehmender Extension $\left(\alpha<0^{\circ}\right)$ nimmt die Verkippung der Schraubachsen in $z-$ und $x$ Richtung bis auf $5,7^{\circ}$ in maximaler Ex-

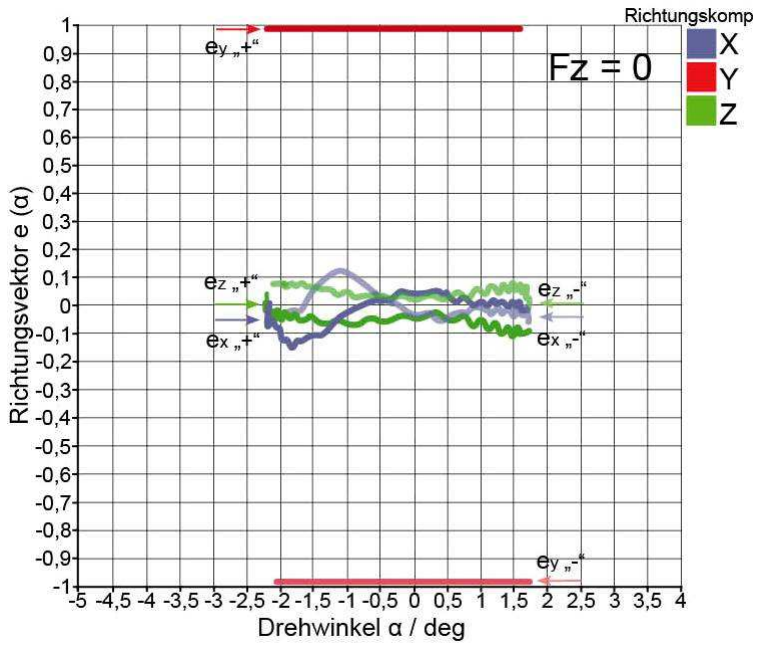

Abb. 3.3.4: Die Komponenten des Richtungsvektors $\underline{e}$ (Th2/3 A) in Abhängigkeit des Drehwinkels $\alpha$ für ein kompletten Messzyklus. tensionslage zu (Abb. 3.3.4). 
Schraubsteigung $\tau(\alpha)$

Die Schraubsteigung $T(\alpha)$ ist in der Flexion klein. Es handelt sich also um eine ebene Bewegung. In der Extension nimmt die momentane Schraubsteigung $\tau(\alpha)$ bis $-0,4 \mathrm{~mm} /$ Grad ab. Die Bandscheibe wird geringfügig komprimiert (Abb.

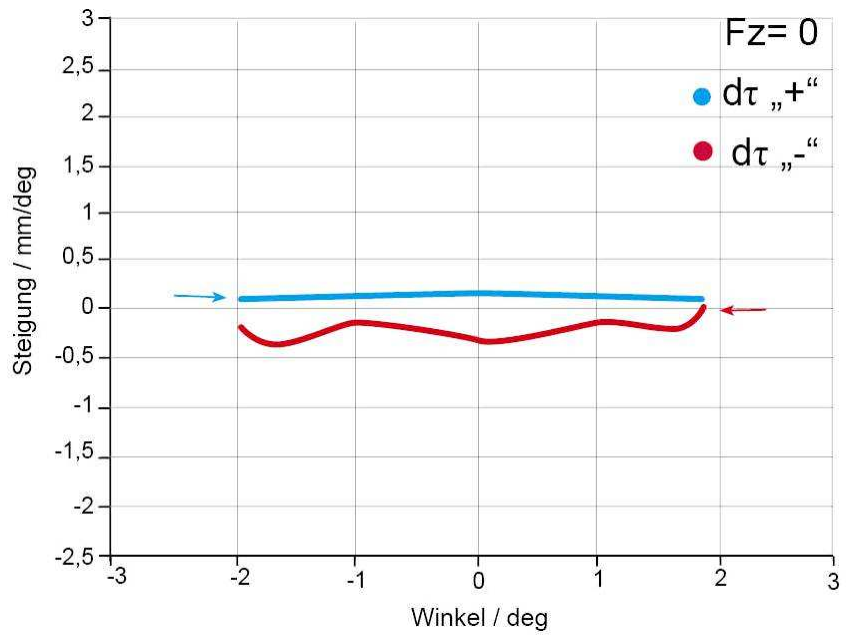

Abb. 3.3.5: Die momentane Schraubsteigung $\tau(\alpha)$ in Abhängigkeit des Drehwinkels $\alpha$.

Für jede Vorlastvariation werden sowohl die Richtungskomponente der IHA als auch die momentane Schraubsteigung bestimmt. Qualitative Veränderungen sind gering. Eine Erhöhung der Vorlast auf $400 \mathrm{~N}$ verändert alle Schraubachsparametern von Th 2/3 A unwesentlich. Da es keine nennenswerte Unterschiede im Verlauf der jeweiligen Schraubsteigung $\tau=\tau(\alpha)$ und des Richtungsvektors $\underline{e}(\alpha)$ zu geben scheint, wird eine axiale Vorlast $F_{z}=200 \mathrm{~N}$ gewählt, um den maximalen Effekt zu beschreiben.

\subsubsection{Verschiebung der Vorlast entlang der $x$-Achse;}

$$
\underline{I}(t)=\left(0, \underline{I}_{Y}(t), 0\right) \wedge F=(0,0,200 N)
$$

Eine Verschiebung der axialen Vorlast von $\mathrm{F}_{\mathrm{Z}}=200 \mathrm{~N}$ in der Sagittalebene verändert die Lage der Schraubachsen nur leicht. Für dorsale und ventrale Vorlasten verschiebt sich die IHA um weitere $5-10 \mathrm{~mm}$ nach kaudal. Für ventrale Vorlasten verkippen die Schraubachsen gering im Winkelbereich von $-2^{\circ}$ bis $1^{\circ}$ und für weit dorsale Vorlasten von $0^{\circ}$ bis $4^{\circ}$. Es gibt keine nennenswerte Unterschiede im Verlauf der jeweiligen Schraubsteigung $\tau=\tau(\alpha)$ und des Richtungsvektors $\underline{\mathrm{e}}(\alpha)$. 
Sukzessive Resektion der Articulationes zygapophysiales Th2/Th3 A

Nach Resektion der rechten Art. zygapophysialis resultiert eine nach kranial offene, v-förmige Rastpolkurve. Die Verkippung des oberen Wirbelkörpers in eine Extensionslage $(\alpha<0)$ führt zu einer Wanderung der IHAs nach kranial in Richtung des verbliebenen Gelenks. In der Flexion $(\alpha>0)$ wandern die momentanen Schraubachsen nach ventro-kranial in Richtung der Zwischenwirbelscheibe (Abb. 3.3.6 A). Für Drehwinkel $\alpha=0^{\circ}-2^{\circ}$ zeigen sich nahezu ortsfeste Schraubachsen ( $\Delta \mathrm{x}=4 \mathrm{~mm}$ und $\Delta \mathrm{z}=2 \mathrm{~mm})$. Nur bei extremen Extensionswinkeln wandern die momentanen Schraubachsen nach dorsal und kaudal.
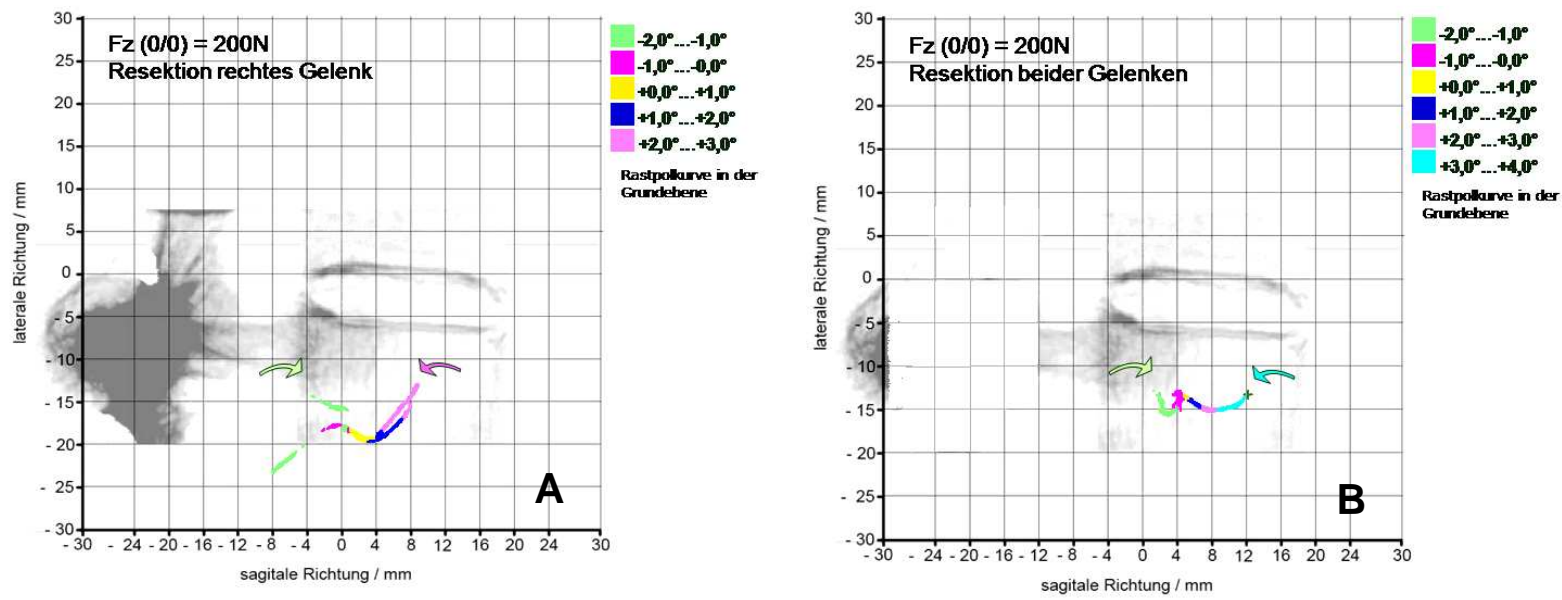

Abb. 3.3.6 A/B: Rastpolkurven von Th 2/3 A nach Resektion des rechten Bogengelenks (A), dann des linken Gelenks (B).

Nach beidseitiger Resektion von Bogengelenken liegen die Rastpolkurven mittig in dem Wirbelkörper des dritten thorakalen Wirbels. Die Rastpolkurven für Extension und Flexion fallen praktisch zusammen (Abb. 3.3.6 B).

Während des Experiments kommt es zu einer Stauchung des Ligamentum interspinale und die Dornfortsätze berühren sich. 


\section{Drehmoment-Drehwinkel-Kennlinien $\alpha(T)$}

Die $\alpha(\mathrm{T})$-Funktion hat unabhängig von Vorlastposition eine sigmoide Form, wobei bei extrem ventralen und dorsalen Kraftangriffen die Drehwinkel-DrehmomentKennlinie eher ein hockeyschlägerförmiges Aussehen besitzt. Bei allen drei Vorlastpositionen kann eine deutliche Hysterese $\Delta \alpha$ gemessen werden (Abb. 3.3.7). Im Einzelnen zeigt sich eine zweifache Reduktion des Bewegungsumfangs für die dorsalen Vorlastpositionen $\left(x_{F}<0\right)$ von $|\alpha|=3,5^{\circ}$ (für zentrale Vorlast) auf $|\alpha|=1,75^{\circ}$ von $50 \%$ und eine geringe Reduktion für die ventrale $\left(x_{F}>0\right)$ Vorlast auf $|\alpha|=2,5^{\circ}$ von $28,5 \%$ des Bewegungsumfanges. Bei dem zentralen Kraftangriff $F_{Z}(0 / 0)$ in der Flexionsbewegung von $\mathrm{T}=0 \rightarrow \mathrm{T}=$ $400 \mathrm{Ncm}$ (positive Rotationsrichtung) beträgt der Bewegungsumfang (ROM) $2,5^{\circ}$. Die $\alpha(T)$-Funktion steigt

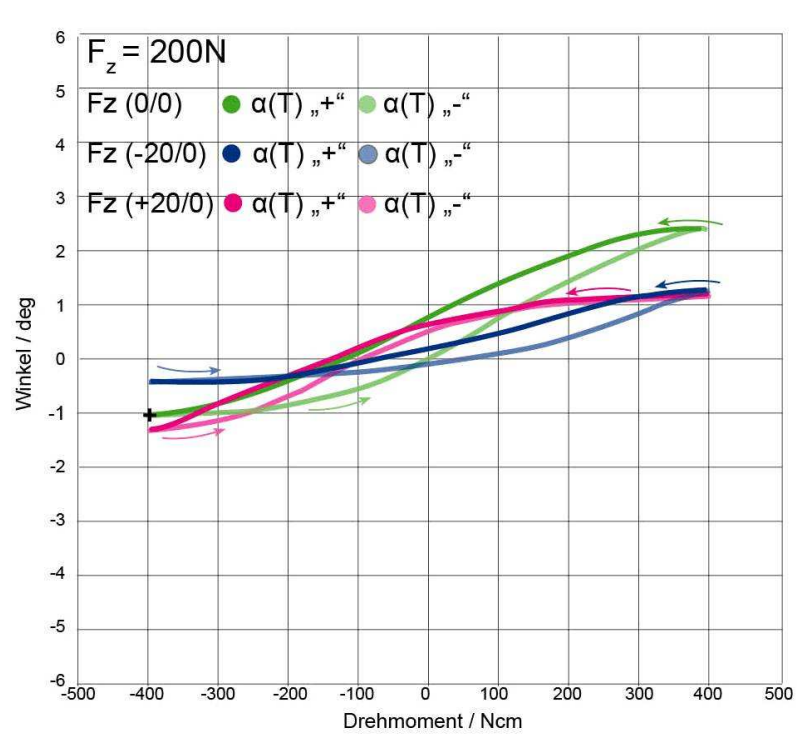

Abb. 3.3.7: Drehwinkel-Drehmoment-Kennlinien. Flexion/ Extension: grün $\mathrm{F}_{Z}(0 / 0)$, pink $\mathrm{F}_{Z}(+20 / 0)$, blau $F_{Z}(-20 / 0)$.

hier fast linear an. Während der Extension von $\mathrm{T}=0 \rightarrow \mathrm{T}=-400 \mathrm{Ncm}$ (negative Rotationsrichtung) zeigt sich nur ein ROM von maximal $1^{\circ}$. Für $\mathrm{T}=0$ war die Hysterese $\Delta \alpha$, bezogen auf den Gesamtbewegungsumfang, nur 0,8 (Abb. 3.3.7).

Die Steifigkeit für eine dorsale Kraftapplikation ist größer als für eine ventrale, wobei die differentielle Steifigkeit bei ventralen Vorlasten sich nur unwesentlich von der Situation nach einem zentralen Kraftangriff unterscheidet.

\section{Sukzessive Resektion der Articulationes zygapophysiales von Th2/Th3 A}

Die Resektion des rechten Bogengelenks erhöht den Rotationsumfang auf $\alpha=$ $3,2^{\circ}$. Die minimale Steifigkeit $B(\alpha=0,59$ ist um $50 \% / \mathrm{Ncm}$ reduziert. Nach dem Entfernen von beider Artt. zygapophysiales erhöht sich der Bewegungsumfang von $|\alpha|=2,5^{\circ}$ für intaktes Segment auf $|\alpha|=5,5^{\circ}$. Die Hysterese $\Delta \alpha(T=0)$ vergrößert sich 
sukzessiv bis auf $1,5^{\circ}$ bei beidseitiger Resektion (Abb. 3.3.8). Die unterschiedliche Steifigkeit des Hin- und Rücklaufs eines Messzykluses ist minimiert.

Die minimale Steifigkeit verkleinert sich zunehmend auf $50 \% \mathrm{Ncm}$ (Abb. 3.3.9).

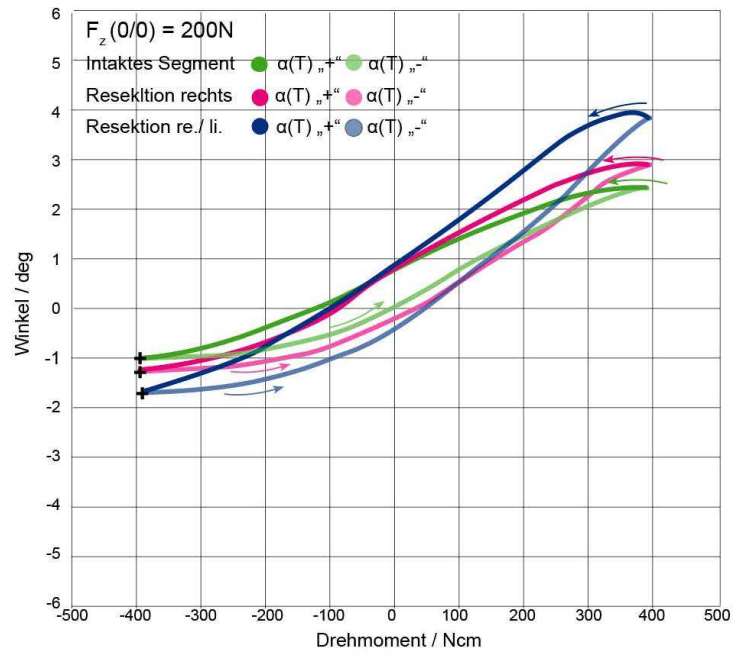

Abb. 3.3.8: Drehmoment-Drehwinkel-Kennlinien von Th 2/3 A nach sukzessiver Resektion des rechten Bogengelenks und beider Gelenke.

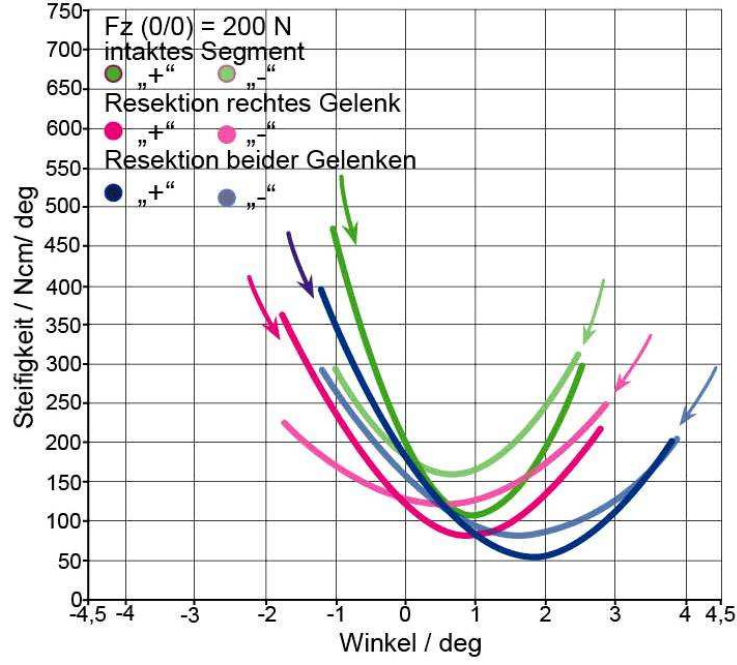

Abb. 3.3.9: Steifigkeit $B_{G}(\alpha)$ von Th $2 / 3$ A nach sukzessiver Resektion des rechten Bogengelenks und beider Gelenke.

\section{$\underline{\text { Richtung der momentanen Schraubachsen e }(\alpha)}$}

Die Schraubachsen liegen parallel und in der Bandscheibenebene $\left(e_{Y}(\alpha) \cong 1\right)$. In der Extensionslage nehmen die $x$ - und z-Komponenten des Richtungsvektors zu, das bedeutet, dass die Schraubachsen in $x$ - und z-Richtung verkippen. Im Vergleich zu Abb. 3.3.4 (S. 119) wird keine Veränderung beobachtet.

\section{Sukzessive Resektion der Articulationes zygapophysiales von Th2/Th3 A}

Nach Resektion des rechten Bogengelenks nehmen die Neigungswinkel der momentanen Schraubachsen in maximaler Extension bis auf 5,7 in z- sowie 4,6 in x-Richtung zu (Abb. 3.3.10 A, S. 124). Die Schraubachsen verkippen auf der Seite des fehlenden Bogengelenks nach „unten“ und nach dorsal. Nach Resektion beider Wirbelbogengelenke liegen die Schraubachsen für $\alpha \geq 1,5^{\circ}$ in einer Ebene parallel zueinander und verkippen nur bei $\alpha \leq 1,5^{\circ}$ nach dorsal. In maximaler Ex- 
tensionsstellung stehen die momentanen Schraubachsen um $6,3^{\circ}$ nach ventral gekippt und geringradig um 2,9 $9^{\circ}$ ach kaudal gericht et (Abb. 3.3.10 B).
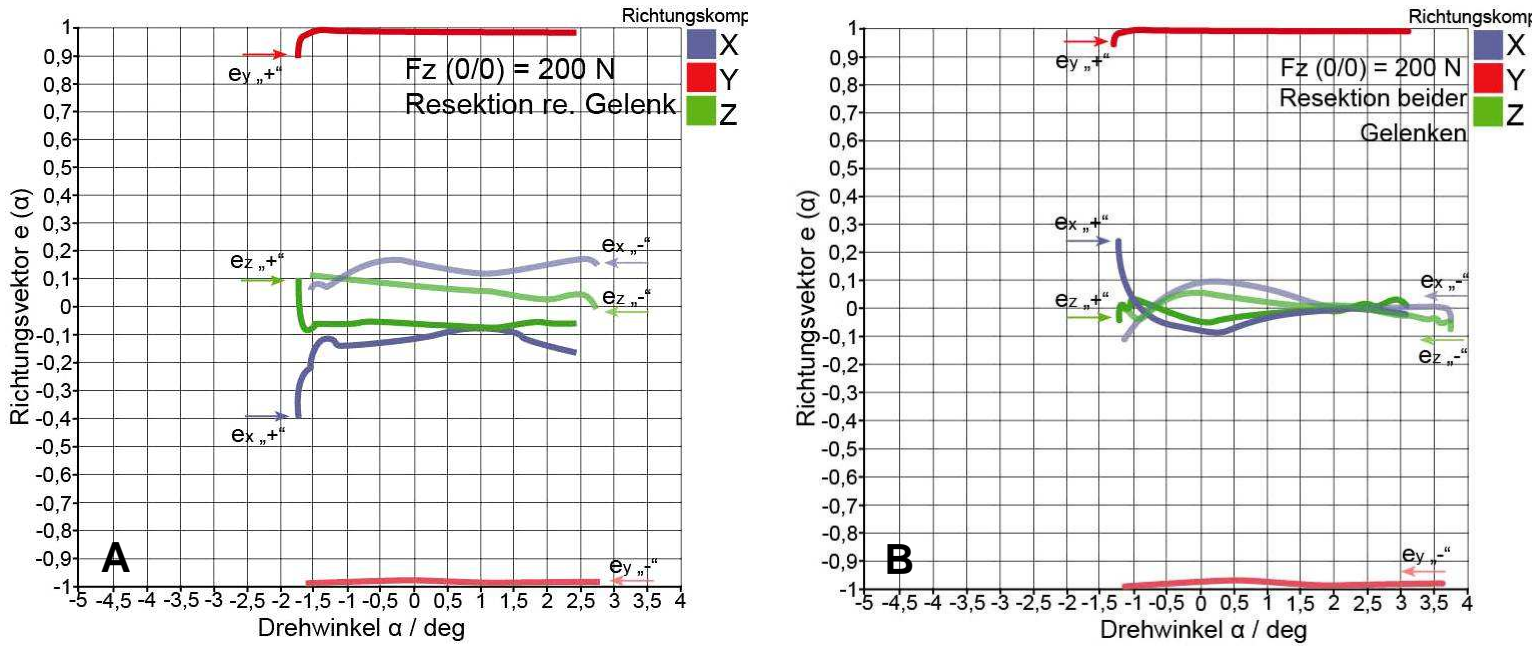

Abb. 3.3.10: A) Die Komponenten des Richtungsvektors nach Resektion des rechten Bogengelenks und B) nach Entfernung beider Gelenke. Die Pfeile geben die Rotationsrichtung an: positive Richtung (Flexion), negative Richtung (Extension).

\section{Schraubsteigung $\tau(\alpha)$}

Die Verschraubung steigt wie erwartet $(\tau \cong 0)$ für Flexion bei ventralen Vorlasten etwas an. Die Schraubsteigung $T(\alpha)$ bei ventralen Vorlasten ähnelt stark der Schraubsteigung der $F_{Z}$ in Widerstandszentrum in der Flexion (Abb. 3.3.5, S. 120). Es handelt sich auch hier um eine ebene Bewegung. Bei dorsaler Vorlast und einem negativen Drehwinkel $\alpha$ kommt es zur gegenläufigen Bewegung; die momentane Schraubsteigung $\tau(\alpha)$ bewegt sich entlang der z-Achse bis $\pm 0,2 \mathrm{~mm} / \mathrm{Grad}$ auf und ab. Die Bandscheibe wird geringfügig komprimiert (Abb. 3.3.11, S. 125).

\section{Sukzessive Resektion der Articulationes zygapophysiales von Th2/Th3 A}

Nach Resektion des rechten Bogengelenks und nach Entfernen des zweiten Gelenks bleibt die Bewegung eben. Die Abbildung 3.3.12 spiegelt letztlich nur den bei der beidseitigen Resektion erhöhten Rotationsumfang wider. 


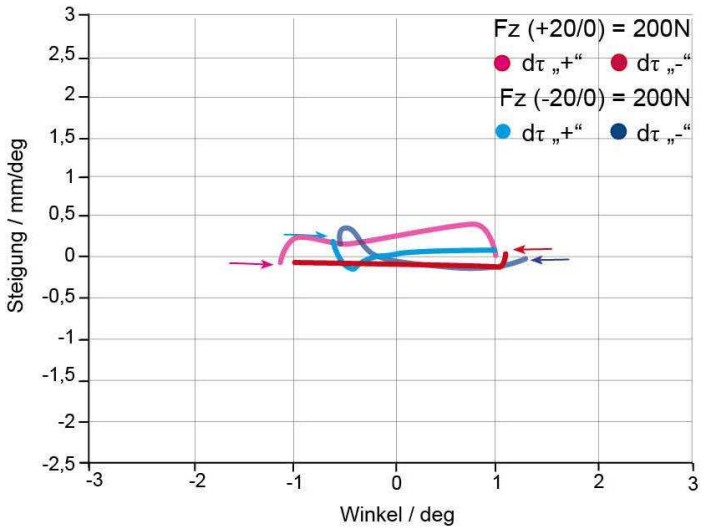

Abb. 3.3.11: Schraubsteigung $\tau(\alpha)$ der momentanen Schraubachsen für Flexion/ Extension: pink $F_{Z}(+20 / 0)$, blau $F_{Z}(-20 / 0)$.

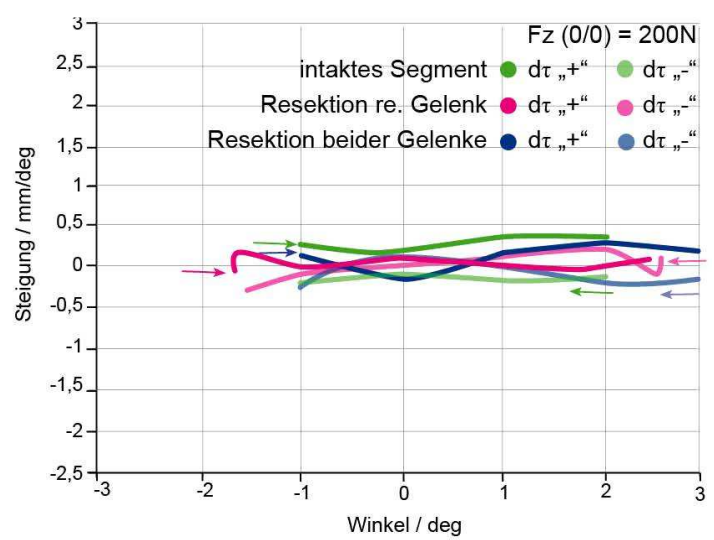

Abb. 3.3.12: Schraubsteigung $\tau(\alpha)$ der momentanen Schraubachsen nach Resektion des rechten Gelenks und dann beider Gelenke.

\subsubsection{Verschiebung der Vorlast parallel zur y-Achse}

$$
I(t)=\left(0, I_{Y}(t), 0\right) \wedge F=(0,0,200 N)
$$

Die Rastpolkurven verändern sich kaum nach einer Verschiebung der Vorlast entlang der y-Achse. Für eine Rotation in beide Drehrichtungen wandern die momentanen Schraubachsen nach dorsal auf einer Bahn unterhalb der Bandscheibe.

Die Drehmoment-Drehwinkel-Kennlinien zeigen einen weniger ausgeprägten Verlauf (Abb. 3.3.13). Der Bewegungsumfang ist für die rechte Vorlastposition mit $|\alpha|=4,4$ etwas größer als für die linke $\left(F_{Z}(0 /+30)\right)$.

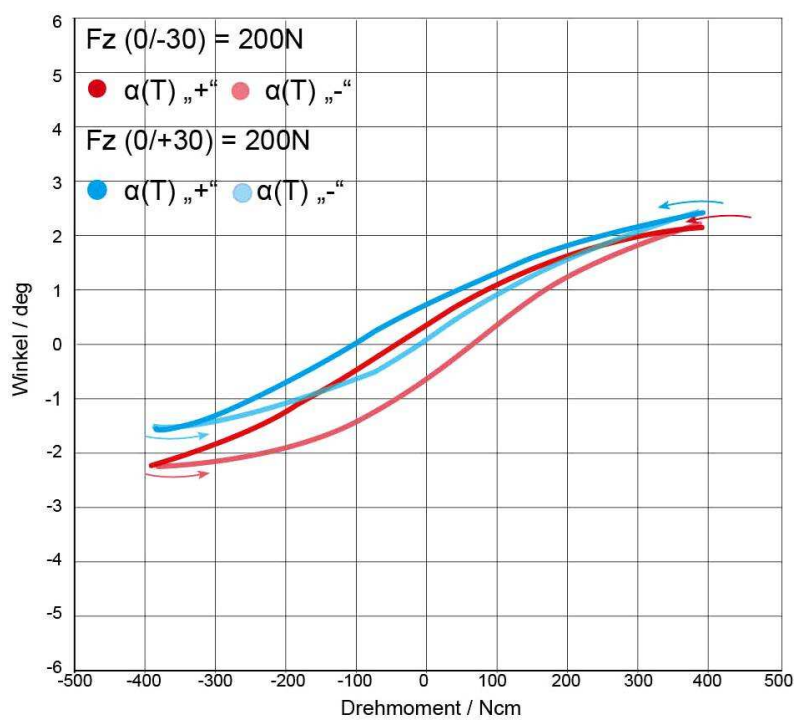

Abb. 3.3.13: Drehmoment-Drehwinkel-Kennlinien von Th $2 / 3 \mathrm{~B}$. Flexion/ Extension für eine laterale Vorlastposition: braun $\mathrm{F}_{Z}(0 /-30)$ und blau $\mathrm{F}_{Z}(0 /+30)$. 
Der Steifigkeitsverlauf $B(\alpha)$ ist leicht verändert. Die minimale Steifigkeit zeigt eine Abhängigkeit von der Positionierung der axialen Vorlast. Bei einer Belastung links ist die minimale Steifigkeit etwas höher als bei einer Rechtsbelastung und liegt bei $90 \% / \mathrm{Ncm}$. Damit ist das Bewegungssegment bei der linksseitigen Belastung steifer als bei der rechtsseitigen.

Für beide Diagramme gilt, dass die Steifigkeit für die positive Drehrichtung höher als für die negative Rotationsrichtung ist (Abb. 3.3.14).

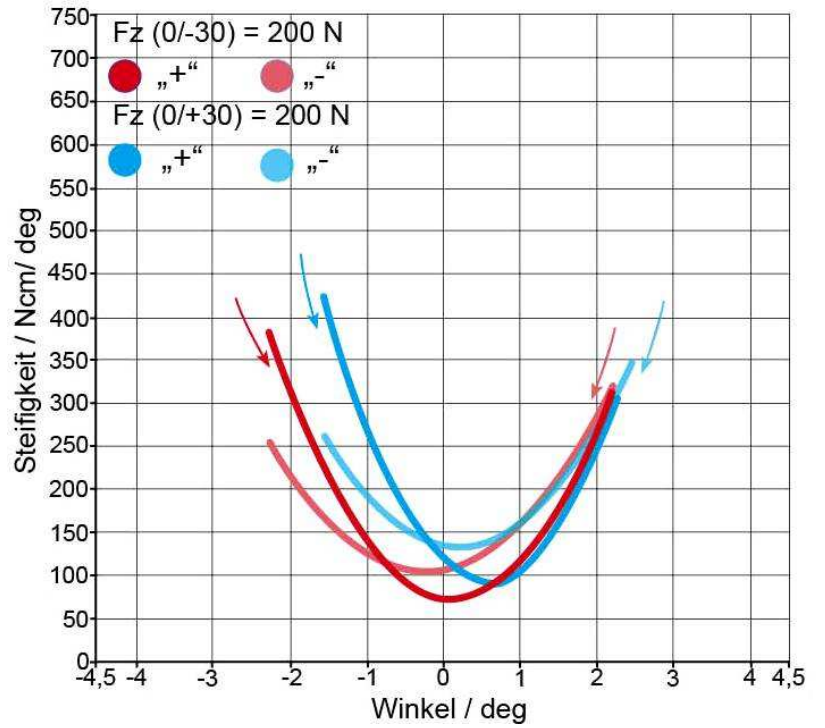

Abb. 3.3.14: Steifigkeit $B_{G}(\alpha)$ von Th $2 / 3$ B. Flexion/ Extension für eine laterale Vorlastposition: braun $\mathrm{F}_{Z}(0 /-30)$ und blau $\mathrm{F}_{Z}(0 /+30)$.

Die Verkippung der Schraubachsen ist größer. Bei einer Vorlast weit rechts verkippt die momentane Schraubachse in der maximalen Extension bis auf 17,5 nach dorsal und in der maximalen Flexion um den Betrag von $43,4^{\circ}$ nach ventral (Abb. 3.3.15 A). Die Schraubachsen verschieben sich auf der z-Achse leicht nach „unten“. Bei einer Vorlast links beträgt die Neigung der momentanen Schraubachse in maximaler Extension 18,7 $7^{\circ}$ nach dorsal und in maximaler Flexion $14,7^{\circ}$ nach ventral (Abb. 3.3.15 B, S. 127). Bei einer rechts-lateralen Vorlast verkippt die IHA von ventral nach dorsal und zurück. Gleichzeitig kommt es zu einer Abwärtsbewegung des Segments. Bei der links-lateralen Belastung verkippt die Schraubachse umgekehrt von dorsal nach ventral und zurück. Das Segment bewegt sich in der maximalen Flexion geringfügig nach „oben“ (Abb. 3.3.15 B). 

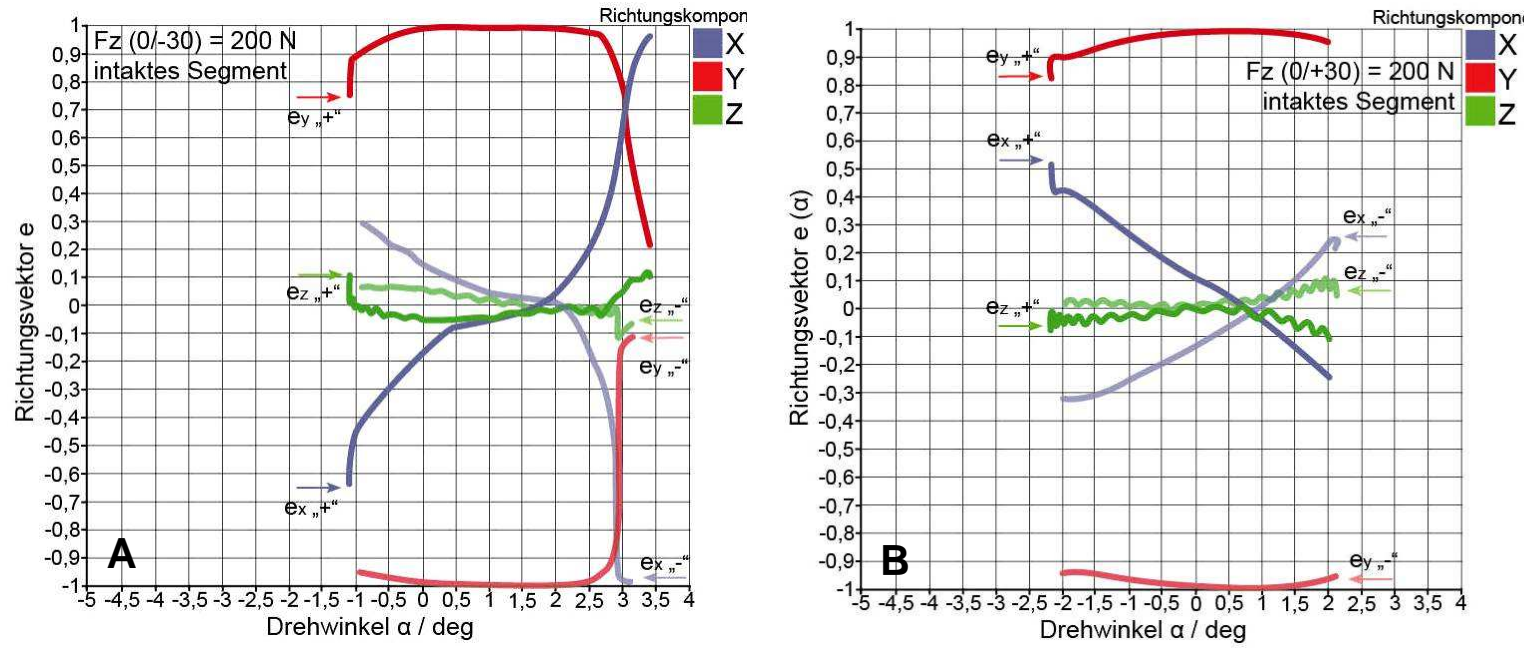

Abb. 3.3.15 A/ B: Richtungsvektor $\underline{e}$ der momentanen Schraubachsen in Abhängigkeit von $\alpha$ für eine laterale Vorlastposition: $A) F_{Z}(0 /-30)$ und $\left.B\right) F_{Z}(0 /+30)$.

Die Abbildung 3.3.16 zeigt das Verhalten der Schraubsteigung. Der Verlauf der jeweiligen Schraubsteigung $\tau=\tau(\alpha)$ spiegelt das Verhalten des Richtungsvektors $\underline{\mathrm{e}}(\alpha)$ wider.

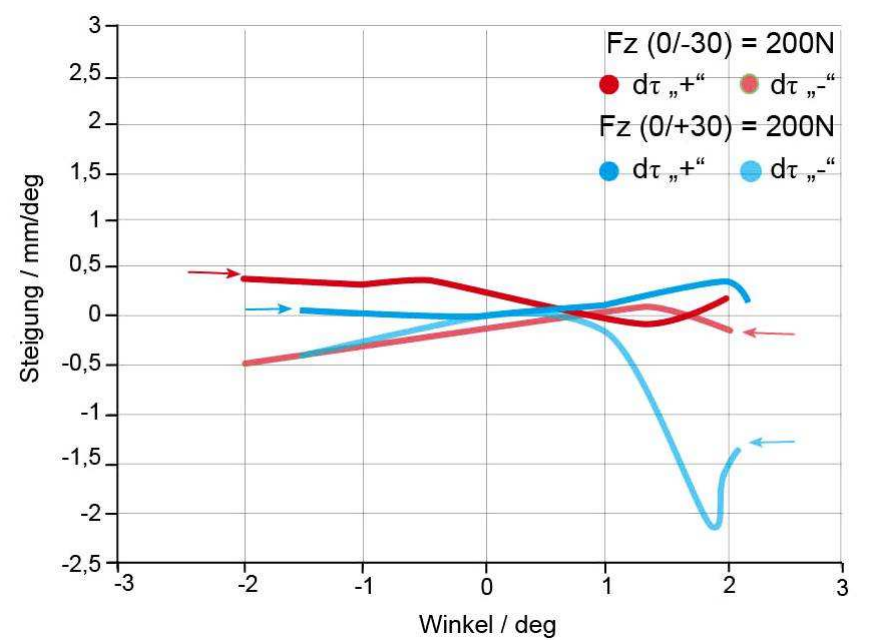

Abb. 3.3.16: Schraubsteigung $\tau(\alpha)$ der momentanen Schraubachsen von Th2/3 A bei verschiedenen Vorlastpositionen.

\section{Sukzessive Resektion der Articulationes zygapophysiales Th2/Th3 A}

Nach Resektion der rechten Art. zygapophysialis resultiert eine nach kranial offene, v-förmige Rastpolkurve. In Abbildung 3.3.17 A (S. 128) liegt der Kraftvektor auf der Seite des vorhandenen Wirbelbogengelenks. Die Extensionsbewegung beginnt etwa 2mm ventral des WZ und knapp oberhalb der Bandscheibe des Th 3/4Bewegungssegments. Die IHA verläuft in einem nach dorsal offenen Bogen und um $6 \mathrm{~mm}$ nach kaudal versetzt. Zu Beginn der Flexionsbewegung springt die Rastpolkurve nach dorsal. Die IHA läuft nach ventro-kranial und endet abrupt auf der 
gleichen Höhe wie zu Beginn der Extension und etwa 3mm nach dorsal versetzt. Die Flexions-, Extensionsbewegung findet ausschließlich im negativen Drehwinkelbereich $\alpha$ von $0^{\circ}$ bis $-3^{\circ}$ statt.
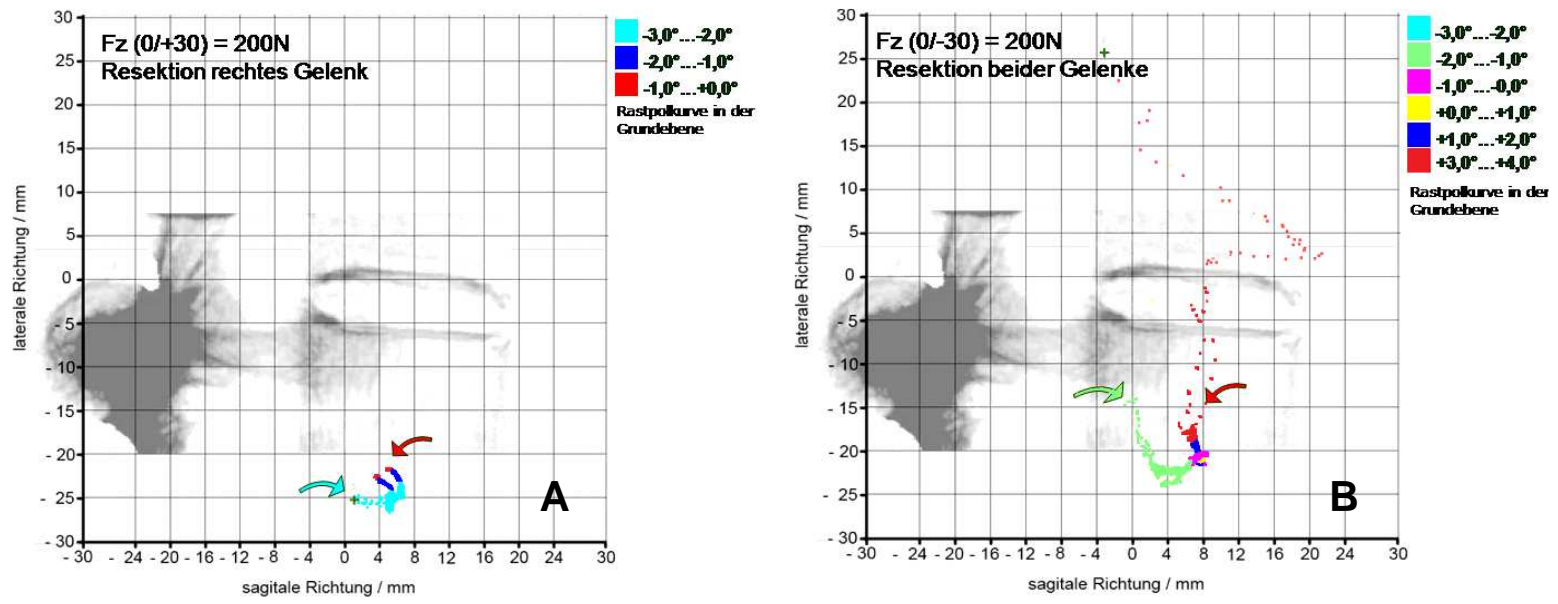

Abb. 3.3.17 A/B: Durchstoßpunkte der Kraftwirkungslinie von $F_{Z}\left(0 / y_{F}\right)$ durch die $x z-$ Referenzebene. Ansicht von rechts: A) Position der Kraftwirkungslinie $F_{Z}(0 /+30)$ nach Resektion des rechten Wirbelbogengelenks, B) Position der Kraftwirkungslinie $F_{Z}(0 /-30)$ nach Resektion beider Wirbelbogengelenke.

Nach beidseitiger Resektion zeigen sich nahezu ortsfeste Schraubachsen ( $\Delta \mathrm{X}=5 \mathrm{~mm}$ und $\Delta \mathrm{z}=5 \mathrm{~mm}$ ) (Abb. 3.3.17 B). Die Rastpolkurve für beide Bewegungsrichtungen zieht nach kranial in die Bandscheibe des Bewegungssegmentes Th 2/3 A. Während des Experiments kommt es zu einer Stauchung des Ligamentum interspinale und die Dornfortsätze berührten sich.

Nach sukzessiver Resektion der Wirbelbogengelenke zeigt sich eine symmetrische $\alpha(T)$-Funktion. Die Abbildung 3.3.18 zeigt im Vergleich erst ein intaktes Segment mit einer rechts lateral angreifenden Kraft $F_{Z}$ und die Situation nach der Resektion der rechten Art. zygapophysialis für den gleichen Wirkungsort der axialen Vor-last. Nach dem Entfernen des linken Wirbelbogengelenks wird zur graphischen

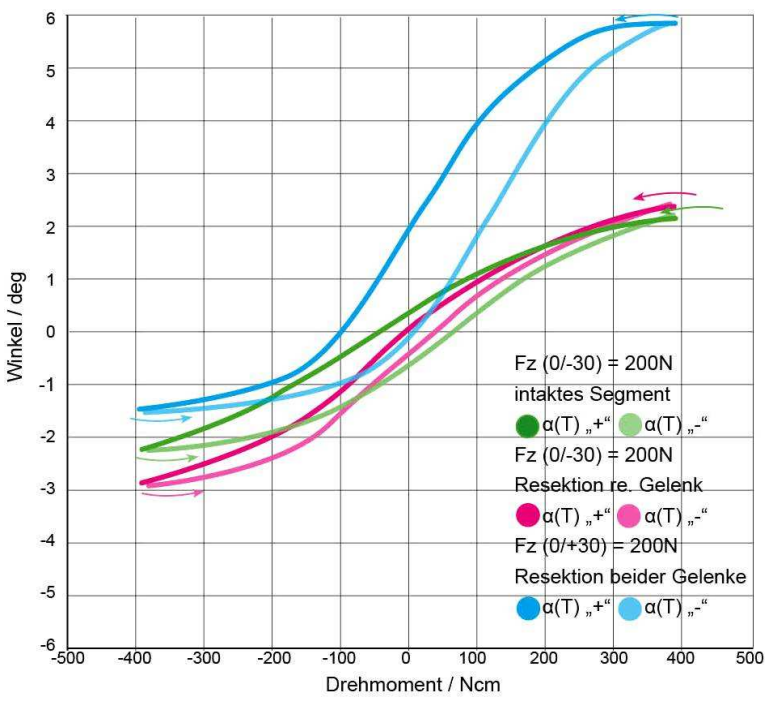

Abb. 3.3.18: Drehwinkel-Drehmoment-KennLinien. Flexion/ Extension nach sukzessiver Resektion von Artt. zygapophysiales. 
Darstellung die Vorlastposition links lateral gewählt. Nach der Resektion des rechten Gelenks erhöht sich der Bewegungsumfang geringfügig um $1^{\circ}$ und die Hysterese $\Delta \alpha$ wird kleiner. Diese Beobachtung gilt für alle betrachtete Vorlastpositionen. Nach Resektion der zweiten Art. zygapophysialis wird eine symmetrische $\alpha(T)$ Abhängigkeit mit einem weit größeren Rotationsbereich von $|\alpha|=7,5^{\circ}$ und eine vergrößerte Hysterese $\Delta \alpha(T=0)$ von $2^{\circ}$ beobachtet (Abb. 3.3.18, S. 128).

Die minimale Steifigkeit bleibt nach einseitiger Resektion des Wirbelbogengelenks unverändert und nimmt nach der zweiten Resektion leicht ab (Abb. 3.3.19).

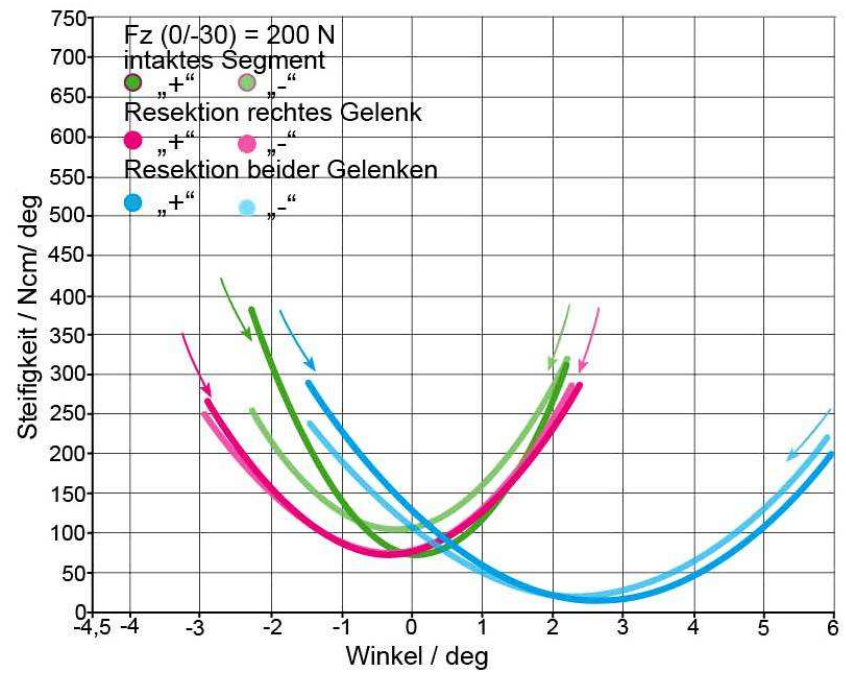

Abb.3.3.19: Die Rotationssteifigkeit $B_{G}(\alpha)$ in Abhängigkeit des Drehwinkels. Flexion/ Extension $200 \mathrm{~N}$ Vorlast.

Die Richtungskomponente der Schraubachsen und die Schraubsteigung $\tau=\tau(\alpha)$ ändern sich nach der Resektion des rechten Gelenks wenig (Vergleich Abb. 3.3.15 A, S. 127). Die Verkippung der Schraubachsen findet in umgekehrter Abfolge statt: Für Flexion im Laufe der Bewegung von ventral nach dorsal und für Extensionsbewegung von dorsal nach ventral (Abb. 3.3.20).

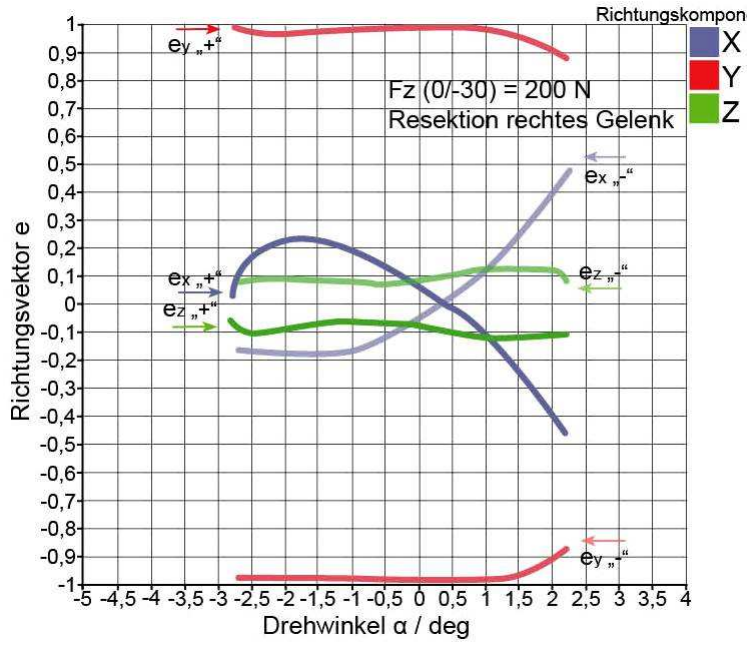

Abb.3.3.20: Die Komponenten des Richtungsvektors nach Resektion des rechten Bogengelenks. 
Nach Resektion des rechten Bogengelenks und nach dem Entfernen des zweiten Gelenks bleibt die Bewegung eben (Abb. 3.3.21).

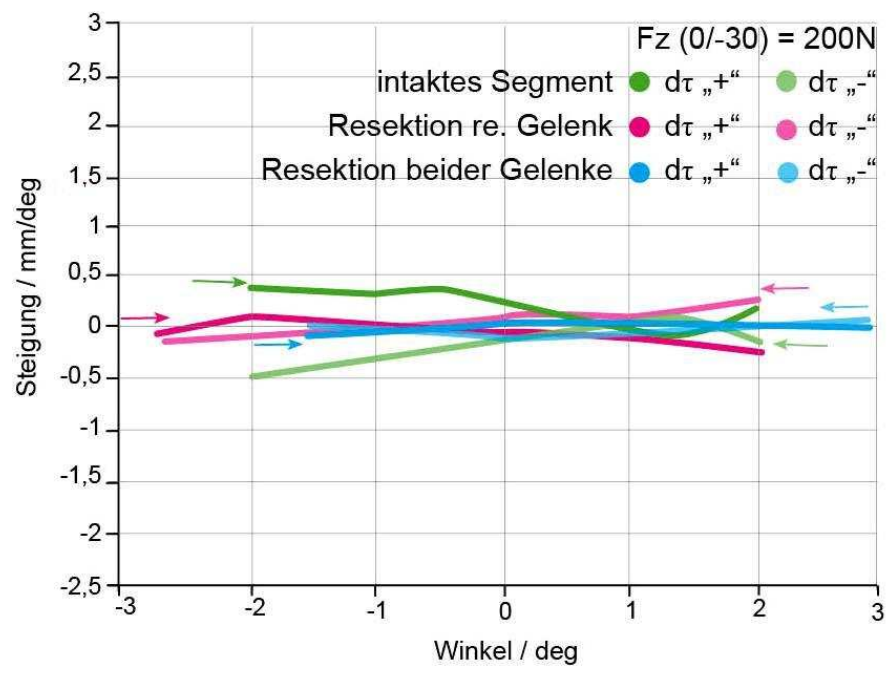

Abb. 3.3.21: Schraubsteigung $\tau(\alpha)$ der momentanen Schraubachse nach Resektion des rechten Gelenks und dann beider Gelenke.

\subsubsection{Bewegungssegment Th2/3 B}

\subsubsection{Applikation eines Drehmoments ohne axiale Vorlast;}

$$
\underline{I}(t)=\left(0, \underline{I}_{Y}(t), 0\right) ; F_{Z}=0
$$

Die Rastpolkurve $\mathrm{r}_{\mathrm{i}}(\alpha)$ des Bewegungssegments Th 2/3 B zeigt keine nennenswerten Unterschiede zum Verlauf der Rastpolkurven des Th 2/3 A-Segments (Kapitel 3.3.1, S. 127). Die IHAs verlaufen im gleichen Umfang wie die am Th 2/3 A zuerst gemessene Werte, nur dass diese um $2 \mathrm{~mm}$ weiter nach dorsal versetzt sind. Die Bewegung ist eben und gleichmäßig. Die Schraubachsen sind nicht ortsfest. 
Drehwinkel- Drehmoment- Kennlinie

Die Abbildung 3.3.22 zeigt die gemessene Drehwinkel-DrehmomentKennlinie bei Applikation eines Drehmoments $T_{Y}$ ohne axiale Vorlast. Die $\alpha(T)$-Funktion ist deutlich sigmoid geformt. Die Hysterese $\Delta \alpha(T=0)$ ist kaum messbar und ist für $\mathrm{T}=0$ gleich $\alpha=0^{\circ}$. Der Bewegungsumfang (ROM) beträgt $3^{\circ}$ mit maximal angreifendem Drehmoment von $400 \mathrm{~N} / \mathrm{cm}$ (Abb. 3.3.22).

\section{$\underline{\text { Rotationssteifigkeit } B_{G}(\alpha)}$}

Die minimale Steifigkeit liegt bei 75 $\mathrm{Ncm} /{ }^{\circ}$. Im Flexions- und Extensionsbereich (negative Drehwinkel steigt die Steifigkeit $B(\alpha)$ bis zu einem Maximum von $510^{\circ} / \mathrm{Ncm}$ an (Abb. 3.3.23).

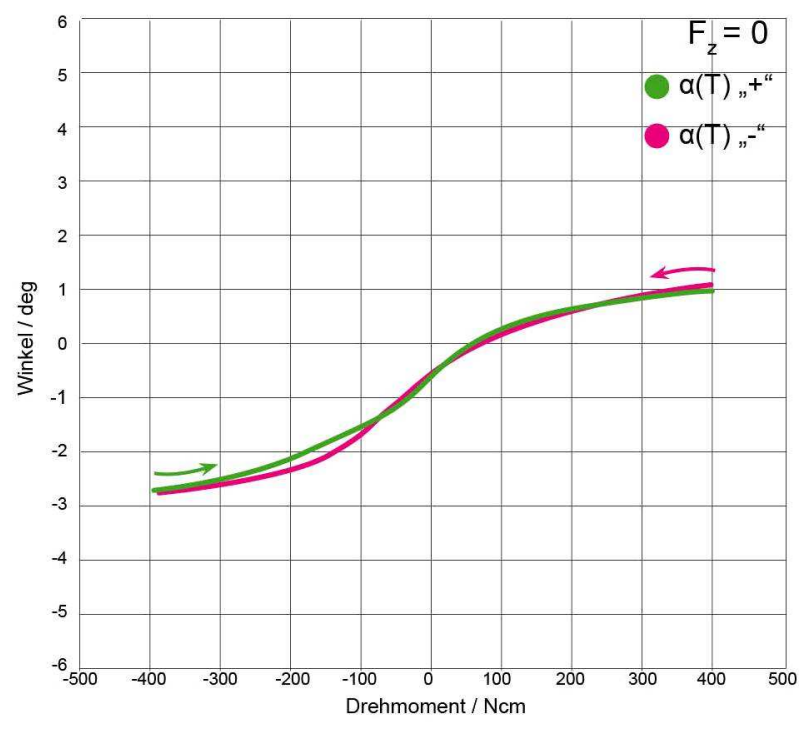

Abb.3.3.22: Drehmoment-Drehwinkel-Kennlinie; die Pfeile zeigen die Laufrichtung an. Positive Richtung: Flexion, negative Richtung: Extension.

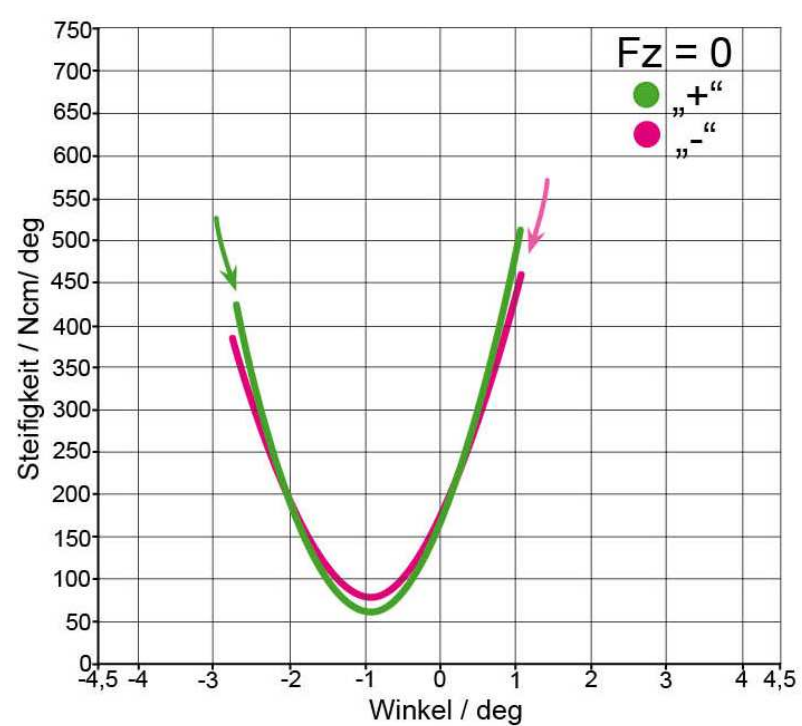

Abb.3.3.23: Die Rotationssteifigkeit $B_{G}(\alpha)$ in Abhängigkeit des Drehwinkels. Erster Halbzyklus grün (Flexion $(,+,)$,$) und zweiter Halbzyklus$ pink (Extension $\left(,{ }^{-“}\right)$ ). 
$\underline{\text { Richtungsvektor der Schraubachse e }(\alpha)}$

Die y-Komponente ist sowohl in Flexions- als auch in Extensionslage $e_{Y}(\alpha)=1$. Somit sind die IHAs parallel zum Drehmoment $T_{Y}$ ausgerichtet. Für den Winkelbereich $\alpha<0^{\circ}$ liegen die Schraubachsen in der Sagittalebene $e_{X}(\alpha)= \pm 0,1$ und für $\alpha>0^{\circ}$ stehen die IHAs parallel zum angreifenden Drehmoment $\mathrm{T}_{\mathrm{Y}}$. Mit zunehmender Extension $\left(\alpha<0^{\circ}\right)$ verkippen die Schraubachsen geringfügig um $1,1^{\circ}$ nach dorsal in $\mathrm{x}$-Richtung.

Die Bewegung ist eben (Abb. 3.3.24).

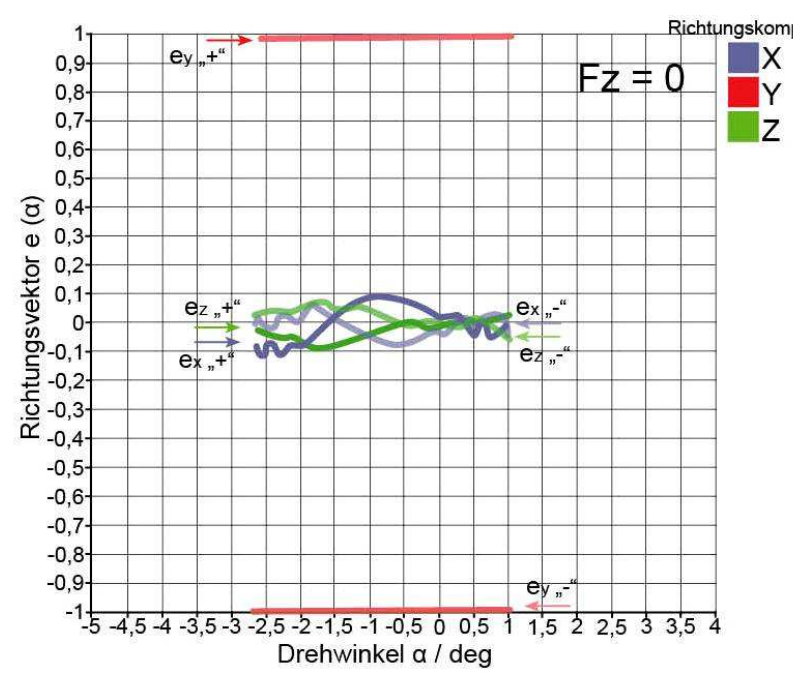

Abb. 3.3.24: Die Komponenten des Richtungsvektors $\underline{e}$ (Th2/3 B) in Abhängigkeit des Dreh winkels $\alpha$ für ein kompletten Messzyklus.

\section{Schraubsteigung $\tau(\alpha)$}

Die Schraubsteigung $T(\alpha)$ steigt in der Flexion bis $\alpha=-1,8^{\circ}$. Die Größenordnung des Versatzes ds des Bewegungssegments entlang der Schraubachse liegt für beide Messzyklen bei $0,35 \mathrm{~mm}$. Für Drehwinkel $\alpha \leq-1,8^{\circ}$ wird die Bandscheibe in Flexion elongiert und für $\alpha \geq-1,8^{\circ}$ um $0,2 \mathrm{~mm}$ komprimiert. In der Extension nimmt die momentane Schraubsteigung $\tau(\alpha)$ fast über den kompletten Bereich ab.

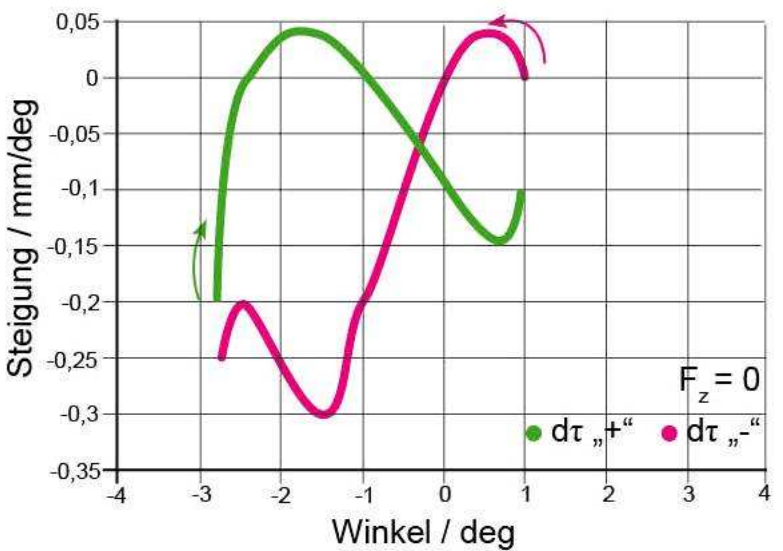

Abb. 3.3.25: Die momentane Schraubsteigung $\tau(\alpha)$ in Abhängigkeit des Drehwinkels $\alpha$. Flexion: grün $(,,+$,$) , Extension: pink (,,“).$

Die Bandscheibe wird um 0,35 mm komprimiert (Abb. 3.3.25). 
Für jede Vorlastvariation werden sowohl die Richtungskomponente der IHA als auch die momentane Schraubsteigung bestimmt. Qualitative Veränderungen sind gering. Bei Erhöhung der Vorlast auf $400 \mathrm{~N}$ verändern sich alle Schraubachsparametern von Th 2/3 B nur unmerklich.

\subsubsection{Verschiebung der Vorlast entlang der $\mathrm{x}$-Achse;}

$$
\underline{I}(t)=\left(0, I_{Y}(t), 0\right) \wedge F=(0,0,200 N)
$$

Die Rastpolkurven verändern sich nach einer Verschiebung der Vorlast entlang der y-Achse in beide Richtungen kaum.

Für dorsale Vorlasten verschiebt sich die IHA um weitere 5-10mm nach dorsal. Für ventrale Vorlasten verschiebt sich die Rastpolkurve um $8 \mathrm{~mm}$ nach kranial in Richtung der Bandscheibe. Für ventrale wie auch für dorsale Vorlasten verkippen die Schraubachsen gering im Winkelbereich von $\alpha= \pm 1,5^{\circ}$.

\section{Drehmoment-Drehwinkel-Kennlinien $\alpha(T)$}

Bei extrem ventralen Vorlasten zeigt sich eine fast lineare Abhängigkeit der $\alpha(T)$-Funktion, die Hysterese $\Delta \alpha \approx 0^{\circ}$. Im Einzelnen kommt es zur zweifachen Reduktion des Bewegungsumfangs für die dorsalen und ventralen Vorlastpositionen $\left(\mathrm{x}_{\mathrm{F}}<0\right)$ von $|\alpha|=3,1^{\circ}$ (für zentrale Vorlast) auf $|\alpha|=1,7^{\circ}$ (Abb. 3.3.26).

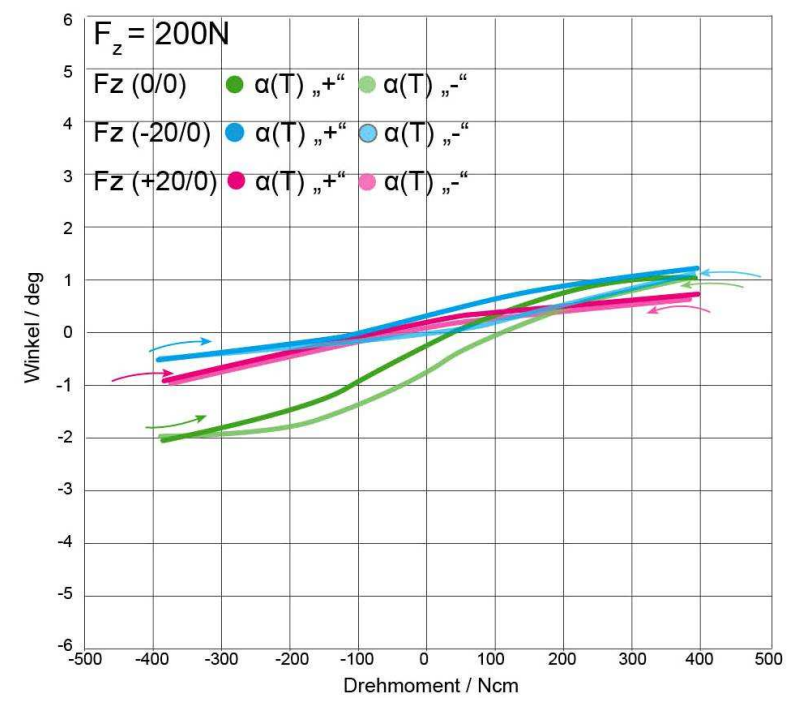

Abb. 3.3.26: Drehwinkel-Drehmoment-Kennlinie. Flexion/ Extension: grün $\mathrm{F}_{Z}(0 / 0)$, pink $\mathrm{F}_{Z}(+30 / 0)$, blau $\mathrm{F}_{Z}(-30 / 0)$. 
Die anfängliche Steifigkeit für eine ventrale und eine dorsale Kraftapplikation ist größer als für die zentrale. Die minimale Steifigkeit steigt von $100 \mathrm{Ncm} /{ }^{\circ}$ für den zentralen Kraftangriff auf 275 - $300 \mathrm{Ncm} /{ }^{\circ}$ für eine dorsale und ventrale Vorlastposition (Abb. 3.3.27)

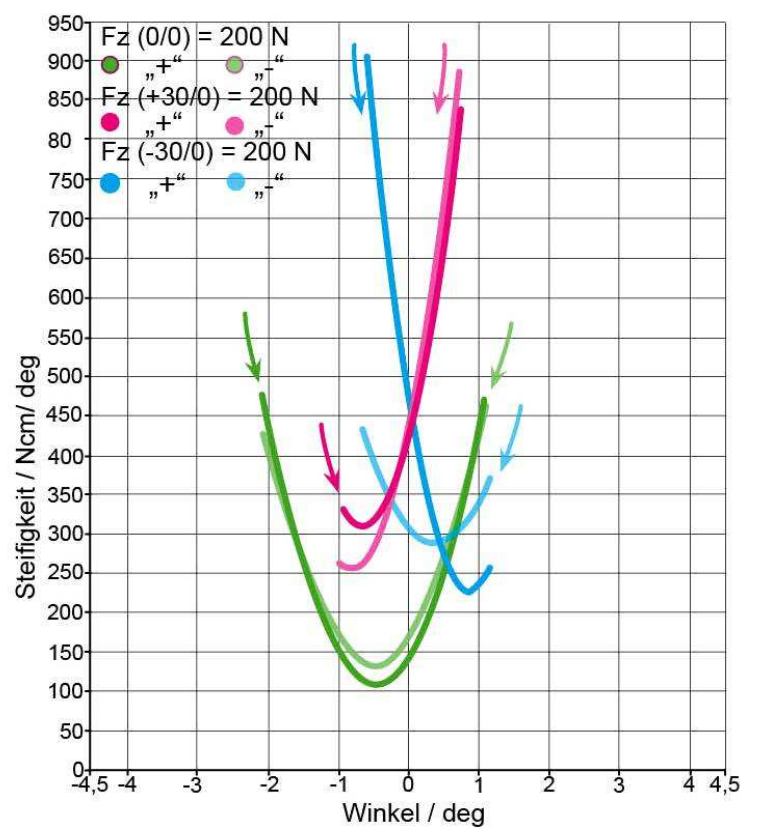

Abb. 3.3.27: Steifigkeit $B_{G}(\alpha)$ von Th $2 / 3 B$. Flexion/ Extension: grün $\mathrm{F}_{Z}(0 / 0)$, pink $\mathrm{F}_{Z}$ $(+30 / 0)$, blau $\mathrm{F}_{Z}(-30 / 0)$.

Es zeigen sich keine nennenswerte Unterschiede im Verlauf der jeweiligen Schraubsteigung $\tau=\tau(\alpha)$ und des Richtungsvektors $\underline{e}(\alpha)$. Die Schraubachsen liegen parallel und in der Bandscheibenebene $\left(e_{Y}(\alpha) \cong 1\right)$. In der Extensionslage nehmen die $\mathrm{x}$ - und $\mathrm{z}$-Komponenten des Richtungsvektors zu, das bedeutet, dass die Schraubachsen in $x$ - und z-Richtung verkippen. Im Vergleich zu Abb. 3.3.24 (S. 132) wird keine nennenswerte Veränderung beobachtet. 


\subsubsection{Verschiebung der Vorlast parallel der $y$-Achse}

$$
I(t)=\left(0, I_{Y}(t), 0\right) \wedge F=(0,0,200 N)
$$

Die Drehmoment-Drehwinkel-Kennlinien zeigen einen weniger ausgeprägten Verlauf. Der Bewegungsumfang (ROM) ist für die rechts laterale Vorlastposition mit $|\alpha|=2,2^{\circ}$ deutlich kleiner als für die links-laterale Vorlast $(|\alpha|=3,59$. Der ROM des Bewegungssegments Th2/3 B mit links-lateral ansetzender $\mathrm{Kraft}\left(\mathrm{F}_{Z}\right.$ $(0 /+30))$ entspricht in etwa dem Bewegungsumfang des Segments bei einer zentralen Kraftapplikation (Abb. 3.3.28).

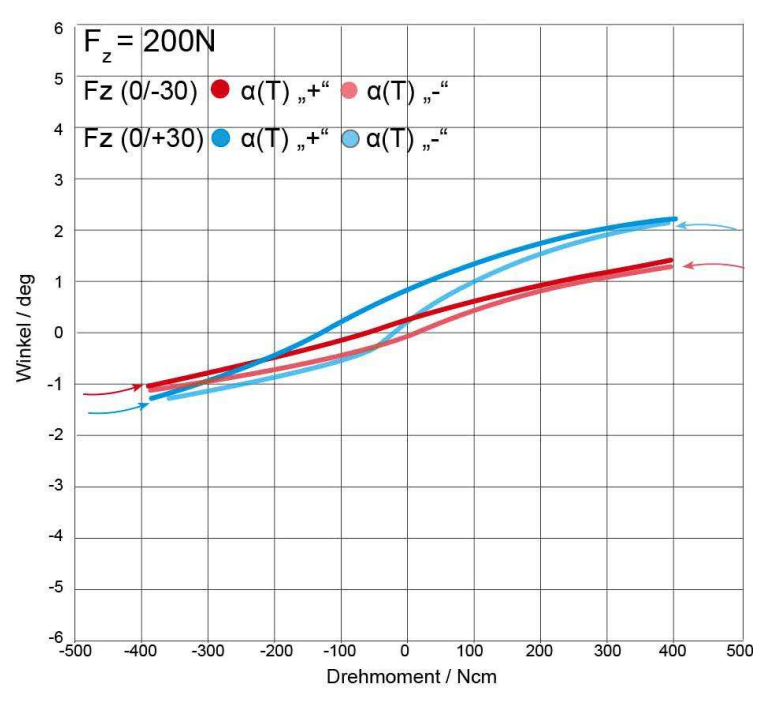

Abb. 3.3.28: Drehmoment-Drehwinkel-Kennlinien von Th2/3 B.

Bei einer Vorlastposition rechts-lateral ist die minimale Steifigkeit höher als bei einer Linksbelastung und liegt bei 235\% Ncm. Für beide Diagramme gilt: Die minimale Steifigkeit für eine negative Drehrichtung (Extension) ist höher als für die positive Rotationsrichtung (Abb. 3.3.29).

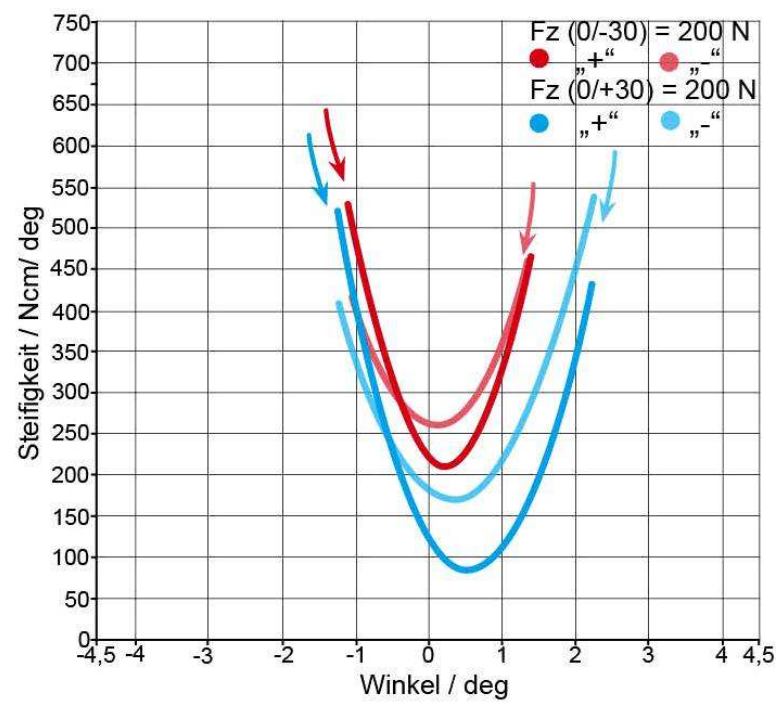

Abb. 3.3.29: Steifigkeit $B_{G}(\alpha)$ von Th2/3 B. 


\subsubsection{Vergleich intakter Bewegungssegmenten Th 2/3 A und Th 2/3 B:}

\begin{tabular}{|c|c|c|}
\hline Merkmal & intaktes Segment Th 2/3 A & intaktes Segment Th 2/3 B \\
\hline \multicolumn{3}{|c|}{ Verhalten der IHAs bei Änderung der Vorlastposition } \\
\hline dorsale Vorlast & $\begin{array}{l}\text { IHAs liegen kaudal und wandern nach } \\
\text { dorsal auf einer Bahn unterhalb der } \\
\text { Bandscheibe. } \\
\text { IHAs nach dorsal verlagert. } \\
\text { IHAs parallel zu Ty. } \\
\text { max. Flexion/ Extension: } \\
\text { leicht nach dorsal gekippt. }\end{array}$ & $\begin{array}{l}\text { IHAs liegen kaudal und wandern nach } \\
\text { dorsal auf einer Bahn unterhalb der } \\
\text { Bandscheibe. } \\
\text { IHAs nach dorsal verlagert. } \\
\text { IHAs parallel zu Ty. } \\
\text { max. Flexion/ Extension: } \\
\text { leicht nach dorsal gekippt. }\end{array}$ \\
\hline ventrale Vorlast & $\begin{array}{l}\text { IHAs liegt kaudal und leicht nach ventral } \\
\text { verlagert. } \\
\text { IHAs parallel zu Ty. } \\
\text { max. Flexion/ Extension: } \\
\text { leicht nach ventral gekippt. }\end{array}$ & $\begin{array}{l}\text { IHAs verschiebt sich nach kranial und } \\
\text { liegt mittig/ zentral. } \\
\text { IHAs parallel zu Ty. } \\
\text { max. Flexion: } \\
\text { senkrecht, parallel zu Ty. } \\
\text { max. Extension: } \\
\text { leicht nach ventral gekippt. }\end{array}$ \\
\hline laterale Vorlast & Keine Änderung der IHAs. & Keine Änderung der IHAs. \\
\hline \multicolumn{3}{|c|}{ Verhalten von ROM und Steifigkeit } \\
\hline dorsale Vorlast & $\begin{array}{l}\text { ROM } \downarrow \\
\text { Hysterese } \downarrow \\
\text { minimale Steifigkeit } \uparrow \\
\end{array}$ & $\begin{array}{l}\text { ROM } \downarrow \\
\text { Hysterese } \downarrow \\
\text { minimale Steifigkeit } \uparrow \\
\end{array}$ \\
\hline ventrale Vorlast & $\begin{array}{l}\text { ROM } \downarrow \\
\text { Hysterese } \downarrow \\
\text { minimale Steifigkeit } \uparrow \text { geringfügig }\end{array}$ & $\begin{array}{l}\text { ROM } \downarrow \\
\text { Hysterese } \downarrow \\
\text { minimale Steifigkeit } \uparrow \text { geringfügig }\end{array}$ \\
\hline laterale Vorlast & $\begin{array}{l}\text { ROM } \uparrow \\
\text { Hysterese } \uparrow \\
\text { minimale Steifigkeit } \downarrow\end{array}$ & $\begin{array}{l}\text { ROM unverändert } \\
\text { Hysterese unverändert } \\
\text { minimale Steifigkeit } \downarrow\end{array}$ \\
\hline
\end{tabular}

Tab.3.4: Zusammenfassung der Ergebnisse der Versuchsreihe „Flexion/Extension“ für Th2/3 A und Th2/3 B. 


\subsubsection{Vergleich intaktes Bewegungssegment und Segment nach der Resektion der rechten Art. zygapophysialis (Th 2/3 A)}

\begin{tabular}{|c|c|c|}
\hline Merkmal & intaktes Segment Th 2/3 A & $\begin{array}{c}\text { Resektion rechten Art. zygapo- } \\
\text { physialis }\end{array}$ \\
\hline \multicolumn{3}{|c|}{ Verhalten der IHAs bei Änderung der Vorlastposition } \\
\hline dorsale Vorlast & $\begin{array}{l}\text { IHAs liegen kaudal und wandern nach } \\
\text { dorsal unterhalb der Bandscheibe. } \\
\text { IHAs parallel zu Ty. } \\
\text { IHAs nach dorsal verlagert. } \\
\text { max. Flexion/ Extension: } \\
\text { leicht nach dorsal gekippt. }\end{array}$ & $\begin{array}{l}\text { IHAs verlaufen in einem nach krani- } \\
\text { al offenem v-förmigen Bogen. } \\
\text { IHAs parallel zu Ty. } \\
\text { max. Extension: } \\
\text { nach dorso-kranial. } \\
\text { max. Flexion: } \\
\text { nach ventro-kranial. }\end{array}$ \\
\hline ventrale Vorlast & $\begin{array}{l}\text { IHAs liegt kaudal und leicht nach ventral } \\
\text { verlagert. } \\
\text { IHAs parallel zu Ty. } \\
\text { max. Flexion/ Extension: } \\
\text { leicht nachventral gekippt. }\end{array}$ & $\begin{array}{l}\text { Verlauf in einem nach kranial offe- } \\
\text { nem v-förmigen Bogen, leicht nach } \\
\text { ventral verlagert. } \\
\text { IHAs parallel zu Ty. } \\
\text { max. Extension: } \\
\text { nach rechts und dorsal gekippt. } \\
\text { max. Flexion: } \\
\text { nach rechts-ventral gekippt. }\end{array}$ \\
\hline laterale Vorlast & Keine Änderung der IHAs & IHAs nahezu ortsfest \\
\hline \multicolumn{3}{|c|}{ Verhalten von ROM und Steifigkeit } \\
\hline dorsale Vorlast & $\begin{array}{l}\text { ROM } \downarrow \\
\text { Hysterese } \downarrow \\
\text { minimale Steifigkeit } \uparrow\end{array}$ & $\begin{array}{l}\mathrm{ROM} \uparrow \\
\text { Hysterese } \uparrow \\
\text { minimale Steifigkeit unverändert }\end{array}$ \\
\hline ventrale Vorlast & $\begin{array}{l}R O M \downarrow \\
\text { Hysterese } \downarrow \\
\text { minimale Steifigkeit } \uparrow \text { geringfügig }\end{array}$ & $\begin{array}{l}\mathrm{ROM} \uparrow \\
\text { Hysterese } \uparrow \\
\text { minimale Steifigkeit } \downarrow\end{array}$ \\
\hline laterale Vorlast & $\begin{array}{l}\text { ROM } \uparrow \\
\text { Hysterese } \uparrow \\
\text { minimale Steifigkeit } \downarrow\end{array}$ & $\begin{array}{l}\text { ROM } \uparrow \\
\text { Hysterese } \downarrow \\
\text { minimale Steifigkeit unverändert }\end{array}$ \\
\hline
\end{tabular}

Tab.3.5: Zusammenfassung der Ergebnisse der Versuchsreihe „Flexion/ Extension“ für Th2/3 A nach Resektion der rechten Art. zygapophysialis. 


\subsubsection{Vergleich intaktes Bewegungssegment und} Bandscheibe- Bänder- Präparat (Th 2/3 A)

\begin{tabular}{|c|c|c|}
\hline Merkmal & intaktes Segment Th 2/3 A & Bandscheibe-Bänder-Präparat \\
\hline \multicolumn{3}{|c|}{ Verhalten der IHAs bei Änderung der Vorlastposition } \\
\hline dorsale Vorlast & $\begin{array}{l}\text { IHAs liegen kaudal und wandern nach } \\
\text { dorsal unterhalb der Bandscheibe. } \\
\text { IHAs parallel zu Ty } \\
\text { IHAs nach dorsal verlagert. } \\
\text { max. Flexion /Extension: } \\
\text { leicht nach dorsal gekippt. }\end{array}$ & $\begin{array}{l}\text { IHAs konstantes Verhalten. } \\
\text { IHAs liegen mittig in dem Wirbel- } \\
\text { körper Th } 3 . \\
\text { parallel zu Ty }\end{array}$ \\
\hline ventrale Vorlast & $\begin{array}{l}\text { IHAs liegen kaudal und leicht nach ventral } \\
\text { verlagert. } \\
\text { IHAs parallel zu Ty } \\
\text { max. Flexion /Extension: } \\
\text { leicht nach ventral gekippt. }\end{array}$ & $\begin{array}{l}\text { IHAs konstantes Verhalten. } \\
\text { IHAs liegt zentral und ortsfest in } \\
\text { dem Wirbelkörper Th } 3 \\
\text { parallel zu Ty }\end{array}$ \\
\hline laterale Vorlast & Keine Änderung der IHAs & $\begin{array}{l}\text { IHAs minimal zur Seite der Belast- } \\
\text { ung verschoben. }\end{array}$ \\
\hline \multicolumn{3}{|c|}{ Verhalten von ROM und Steifigkeit } \\
\hline dorsale Vorlast & $\begin{array}{l}\text { ROM } \downarrow \\
\text { Hysterese } \downarrow \\
\text { minimale Steifigkeit } \uparrow\end{array}$ & $\begin{array}{l}\mathrm{ROM} \uparrow \\
\text { Hysterese } \uparrow \\
\text { minimale Steifigkeit } \downarrow\end{array}$ \\
\hline ventrale Vorlast & $\begin{array}{l}\text { ROM } \downarrow \\
\text { Hysterese } \downarrow \\
\text { minimale Steifigkeit } \uparrow \text { geringfügig }\end{array}$ & $\begin{array}{l}\text { ROM } \uparrow \\
\text { Hysterese } \uparrow \\
\text { minimale Steifigkeit } \downarrow\end{array}$ \\
\hline laterale Vorlast & $\begin{array}{l}\text { ROM } \uparrow \\
\text { Hysterese } \uparrow \\
\text { minimale Steifigkeit } \downarrow\end{array}$ & $\begin{array}{l}\mathrm{ROM} \uparrow \\
\text { Hysterese } \uparrow \\
\text { minimale Steifigkeit } \downarrow\end{array}$ \\
\hline
\end{tabular}

Tab.3.6: Zusammenfassung der Ergebnisse der Versuchsreihe „Flexion/ Extension“ für Th2/3 A nach Resektion beider Artt. zygapophysiales. 


\subsection{Lateralflexion $\underline{I}(t)=\left(I_{x}(t), 0,0\right)$}

Im Versuch „Lateralflexion“ wird ein zyklisches, sich linear veränderndes Drehmoment $\underline{I}(t)=\left(\underline{I}_{x}(t), 0,0\right)$ von $500 \mathrm{Ncm}$ für ein intaktes Segment aufgebracht (Abb. 2.5.3 A, S. 59). Für jedes Segment wird erst ein Drehmoment $T_{x}$ ohne axiale Vorlast appliziert und die resultierende Bewegung aufgezeichnet. Für alle weitere Messungen wird eine axiale Vorlast $F_{z}=200 \mathrm{~N}$ gewählt und der Ort ihrer Kraftwirkungslinie entlang der $\mathrm{x}$ - und $\mathrm{y}$-Achse verschoben. Anschließend erfolgt die sukzessive Resektion des rechten und des linken Wirbelbogengelenks, wobei das Drehmoment $T_{x}$ von $500 \mathrm{Ncm}$ auf $400 \mathrm{Ncm}$ reduziert wird, um das Bewegungssegment nicht zu stark zu beanspruchen. Diese Untersuchung ist auch in diesem Experiment nur auf das Segment Th2/3 A beschränkt.

\subsubsection{Bewegungssegment Th 2/3 A}

\subsubsection{Applikation eines Drehmoments ohne axiale/ mit axialer Vorlast; $\underline{I}(t)=(\underline{T} x(t), 0,0) \wedge F_{Z}=(0,0,0)$}

Rastpolkurven

Die Durchstoßpunkte der Rastpolkurven wandern mittig und kaudal auf der Höhe des vierten thorakalen Wirbels. Die momentane Schraubachsen verlaufen in einem flachen nach oben offenen Bogen über ein Bereich von $\Delta y=16 \mathrm{~mm}$ und $\Delta z=10 \mathrm{~mm}$ (Abb. 3.4.1 A, S. 140). Zu Beginn der Rechtsneigung (negative Rotationsrichtung) macht die momentane Schraubachse einen kleinen Schlenker nach kaudal und richtet sich dann für $\alpha>-1,5^{\circ}$ wieder nach kranial auf. Bei der Bewegungsumkehr aus maximaler Rechtsneigung springt die Rastpolkurve um $4 \mathrm{~mm}$ nach rechts und um $5 \mathrm{~mm}$ nach oben. Die Linksneigung setzt ein. Der Verlauf von Rastpolkurven in der Linksneigung fällt mit der Bahn der Rechtsneigung praktisch zusammen. Die Abbildung 3.4.1.B (S. 140) zeigt exemplarisch die minimalen Änderungen im Verlauf von momentanen Schraubachsen bei einer Erhöhung der Vorlast auf $200 \mathrm{~N}$. Eine weitere Vorlasterhöhung auf $400 \mathrm{~N}$ veränderte $\mathrm{R}_{\mathrm{i}}(\alpha)$ nicht. 

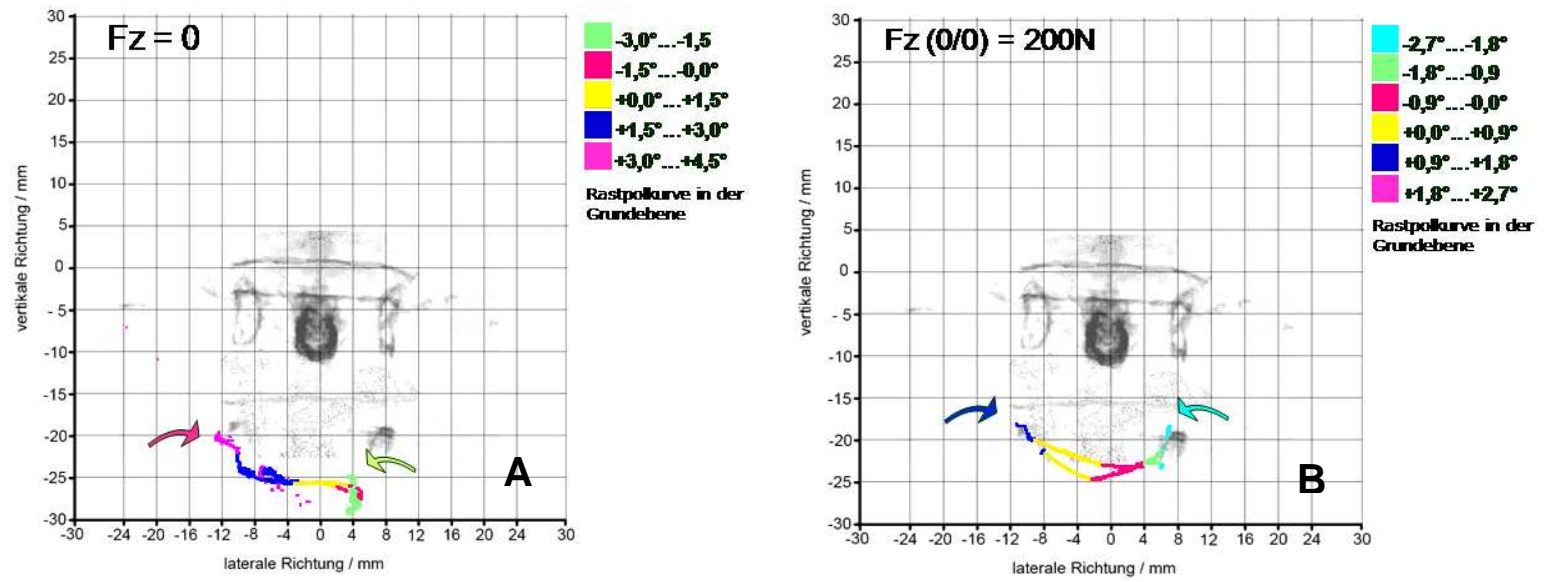

Abb. 3.4.1 Th 2/3 A: Durchstoßpunkte $R_{i}(\alpha)$ der momentanen Schraubachsen für eine Drehung in positiver und negativer Drehrichtung. A) für $F_{Z}=0$; B) $F_{Z}=200 N$.

Die Pfeile geben die Laufrichtung der Rastpolkurve und die Rotationsrichtung des oberen Wirbels an. Ansicht von ventral.

\section{Drehwinkel- Drehmoment- Kennlinie}

Der Bewegungsumfang beträgt $|\alpha|=$

$6,1^{\circ}$ bei einem maximal angreifenden Drehmoment von $500 \mathrm{Ncm}$. Die Drehmoment-Drehwinkel-Kennlinie ist sigmoid. Sie zeigt eine kaum messbare Hysterese $\Delta \alpha(T=0)$. Für $T=0$ war die Hysterese $\alpha=0,1^{\circ}$. Die $\alpha(T)$-Funktion ist symmetrisch bezüglich der $y$-Achse, das heißt, die Bewegung ist eben und gleichmäßig (Abb. 3.4.2).

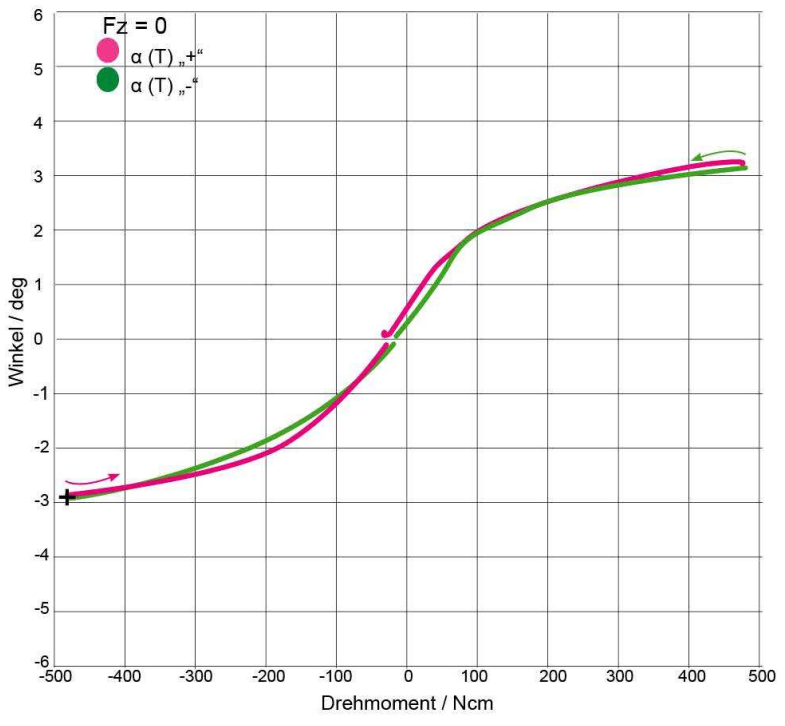

Abb.3.4.2: Drehmoment-Drehwinkel-Kennlinie; die Pfeile zeigen die Laufrichtung an. Linksneigung: positive und Rechtsneigung: negative Richtung. 


\section{$\underline{\text { Rotationssteifigkeit } B_{G}(\alpha)}$}

Die Abbildung 3.4.3 zeigt die Steifigkeits-Drehwinkel-Funktion mit einer minimalen Steifigkeit $B(\alpha)=25-35 \%$ Ncm. In Rechts-, sowie in Linksdrehung (negative Drehwinkel $\alpha$ ), steigt die Steifigkeit $B(\alpha)$ bis zu einem Maximum von $400 \% / \mathrm{Ncm}$ an. Die beiden Halbzyklen haben einen fast identischen Verlauf.

Es wird eine Steifigkeit von $375 \mathrm{Ncm} /{ }^{\circ}$ am Anfang der Bewegung (Pfeil) und die leicht erhöhte Steifigkeit von $400 \mathrm{Ncm}^{\circ}$ am Ende der Bewegung gemessen.

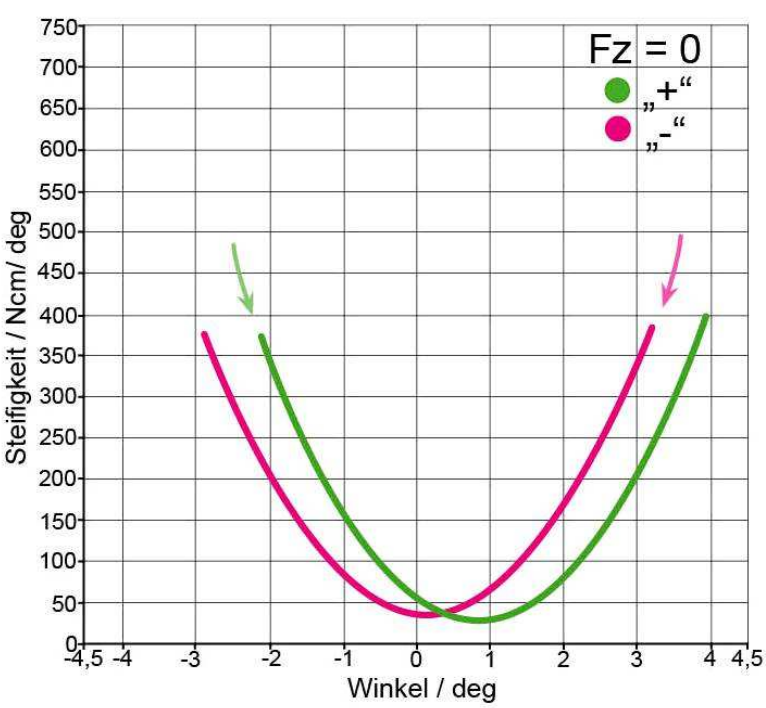

Abb.3.4.3: Die Rotationssteifigkeit $B_{G}(\alpha)$ in Abhängigkeit des Drehwinkels.

Der erste Halbzyklus: grün; (Linksneigung $(,+)$,$) und zweiter Halbzyklus pink; (Rechtsnei-$ gung $(,,-“))$.

\section{Richtungsvektor der Schraubachse e $(\alpha)$}

Die $x$-Komponente ist, sowohl bei Rechts-, als auch bei Linksneigung $e_{X}(\alpha) \cong 1$, somit sind die IHAs parallel zum Drehmoment $\mathrm{T}_{\mathrm{X}}$ ausgerichtet. In maximaler Rechtsneigung sind die IHAs um $7,5^{\circ}$ nach rechts-lateral gekippt, und in maximaler Linksneigung um 5,1 ${ }^{\circ}$ nach links lateral, d.h. dass die momentane Schraubachse zur Seite der jeweiligen Neigungsrichtung verkippt. (Abb. 3.4.4).

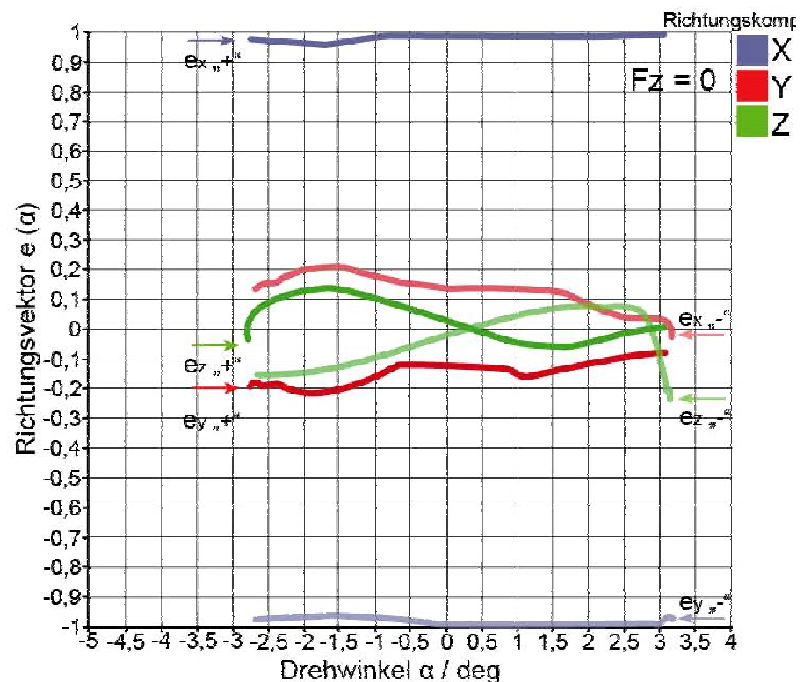

Abb. 3.4.4: Die Komponenten des Richtungsvektors $\underline{e}$ (Th2/3 A) in Abhängigkeit des Drehwinkels $\alpha$ für einen kompletten Messzyklus. 


\section{Schraubsteigung $\tau(\alpha)$}

Die Schraubsteigung $T(\alpha)$ ist in der Lateralflexion klein. Es handelt sich also um eine ebene Bewegung. In der Lateralflexion bewegt sich die momentane Schraubsteigung $\tau(\alpha)$ im Bereich bis $\pm 0,045 \mathrm{~mm} /$ Grad auf und ab (Abb. 3.4.5).

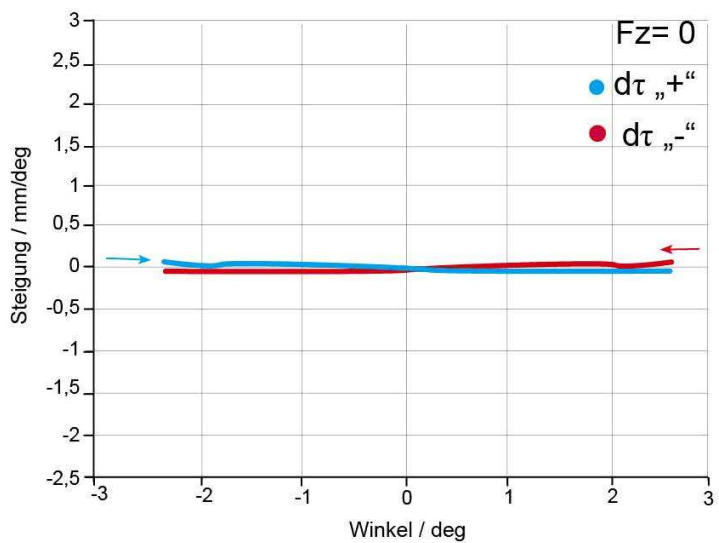

Abb. 3.4.5 : Die momentane Schraubsteigung $\tau(\alpha)$ in Abhängigkeit des Drehwinkels $\alpha$.

Für jede Vorlastvariation werden sowohl die Richtungskomponente der IHA als auch die momentane Schraubsteigung bestimmt. Qualitative Veränderungen sind gering. Bei einer Erhöhung der Vorlast auf $200 \mathrm{~N}$ verändern sich alle vier Schraubachsparametern von Th 2/3 A nur unwesentlich.

\subsubsection{Verschiebung der Vorlast entlang der $x$-Achse;}

$$
\underline{I}(t)=\left(I_{x}(t), 0,0\right) \wedge F=(0,0,200 N)
$$

\section{$\underline{\text { Rastpolkurven }}$}

Bei einer Positionierung der Kraftwirkungslinie der Vorlast dorsal des Widerstandszentrums $W_{Y}$ in (-30/0) sind die Rastpolkurven für einen Hin- und Rücklauf innerhalb eines Messzyklus identisch. Es kann keine Änderung der IHAs zur zentralen Vorlastposition gemessen werden. Es resultiert eine enger geschwungene Rastpolkurve, die im Vergleich zur dorsalen Vorlasten

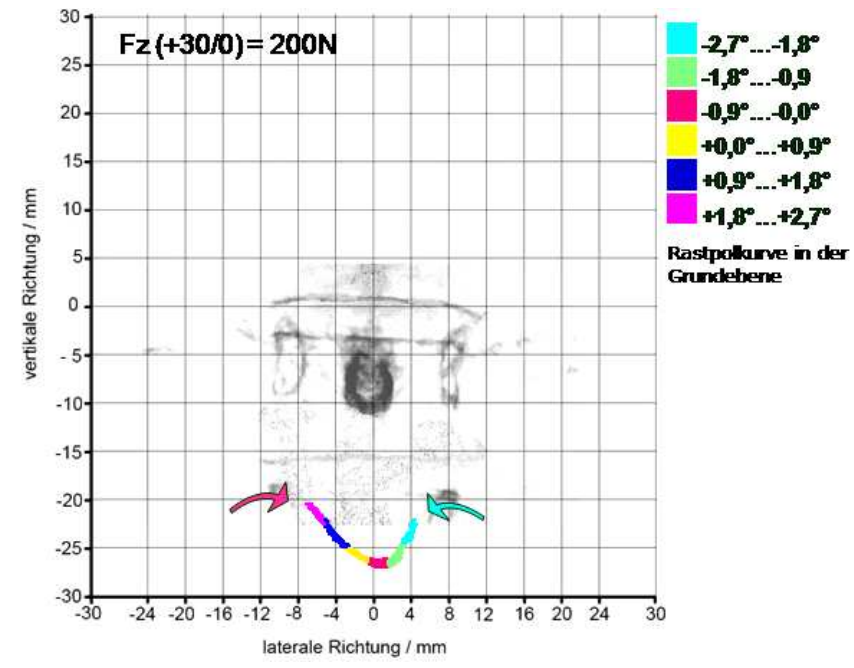

Abb. 3.4.6 Th 2/3 A: Durchstoßpunkte $R_{i}(\alpha)$ der momentanen Schraubachsen für eine Drehung in positiver und negativer Drehrichtung für eine axiale Vorlast $F_{Z}(+30 / 0)=200 \mathrm{~N}$. Ansicht von ventral. 
schmaler und nach unten konkaver wird. Sie dehnt sich über einen Bereich von $\Delta \mathrm{y}=10 \mathrm{~mm}$ und $\Delta \mathrm{z}=12 \mathrm{~mm}$ aus (Abb. 3.4.6).

Bei allen gemessenen Vorlastpositionen sind die Drehwinkelangaben gleichmäßig über die Rastpolkurve verteilt. Damit bleibt die Wanderungsgeschwindigkeit der momentanen Schraubachsen über den kompletten Messbereich annähernd konstant.

\section{Sukzessive Resektion der Articulationes zygapophysiales von Th2/Th3 A}

Die Rastpolkurven zeigen nach der Entfernung des rechten Bogengelenks einen qualitativ ähnlichen Verlauf. Die momentanen Schraubachsen verschieben sich bei zentraler Vorlast um $6 \mathrm{~mm}$ nach kranial. Für eine Neigung nach rechts wandert die Rastpolkurve von links oben nach rechts unten über einen Bereich von $\Delta y=7 \mathrm{~mm}$ und $\Delta \mathrm{z}=16 \mathrm{~mm}$, und in der Linksneigung wandert sie von rechts oben nach links unten.
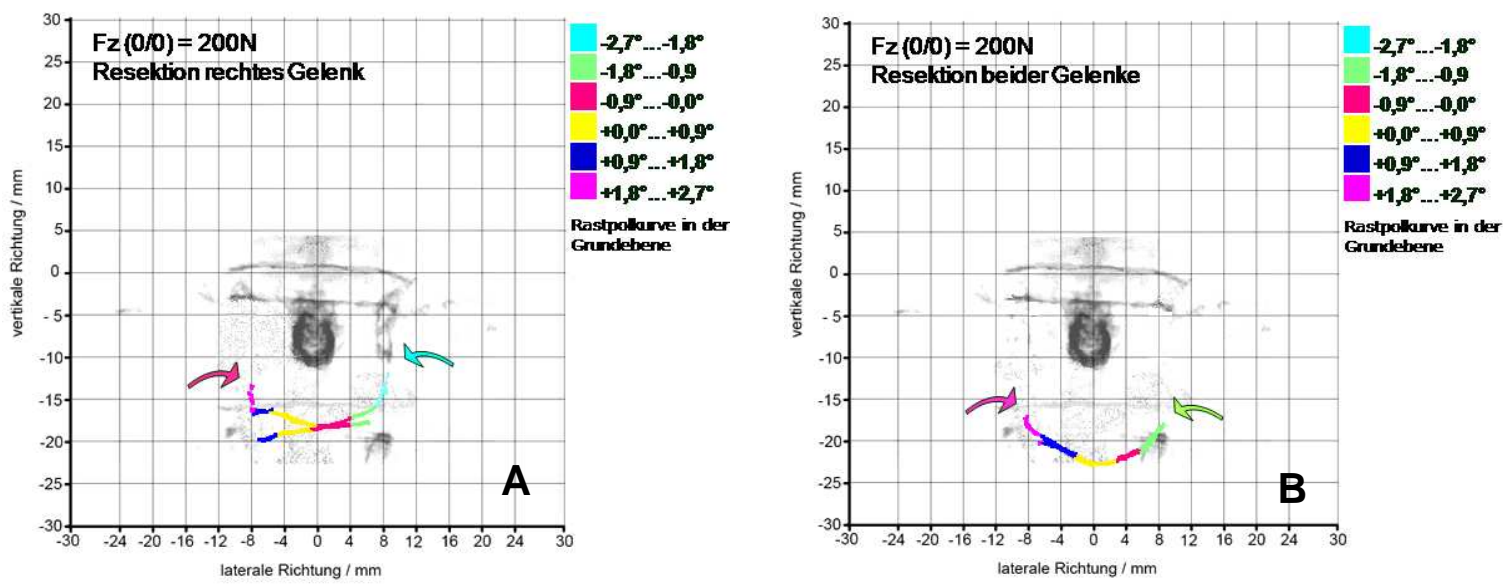

Abb. 3.4.7 A/B: Rastpolkurven von Th2/3 A nach sukzessiver Resektion der Artt. Zygapophysiales $A$ ) die Durchstoßpunkte $R_{i}(\alpha)$ der momentanen Schraubachsen bei $F_{Z}(0 / 0)$ nach Resektion des rechten Bogengelenks; $B$ ) nach Resektion beider Wirbelbogengelenke in Ansicht von ventral.

Die momentanen Schraubachsen für beide Drehrichtungen liegen auf der Höhe der Bandscheibe des Bewegungssegments Th 3/4 (Abb. 3.4.7 A). Bei einem absoluten Drehwinkel $\alpha= \pm 0,9^{\circ}$ lagen die Durchstoßpunkte der momentanen Sc hraubachsen mittig in der Zwischenwirbelscheibe Th 3/4. 
Nach der Resektion des zweiten Bogengelenks zeigte sich der Verlauf der Rastpolkurven dem intakten Segment nach der Applikation einer zentraler Vorlast sehr ähnlich (Abb. 3.4.7 B).

In allen oberen Darstellungen von Rastpolkurven in dem Experiment „Lateralflexion" verlaufen die momentanen Schraubachsen in der anatomischen Projektion des eigebetteten Bewegungssegments von einem Wirbelbogengelenk in die Richtung des anderen Gelenks und zwar auf der Höhe von Artt. zygapophysiales des Junghannsschen Segments Th 3/4 in der Mitte des dritten thorakalen Wirbels.

\section{Drehmoment-Drehwinkel-Kennlinien}

Die $\alpha(T)$-Funktion ist für dorsale Vorlasten eher schlägerformig und für ventrale Vorlastpositionen annähernd sigmoid. Es wird eine kleine Hysterese gemessen. Der Bewegungsumfang (ROM) nimmt bei Verschiebung der Vorlast nach ventral $\left(|\alpha|=5^{\circ}\right)$ um $18 \%$ und für dorsale Vorlastpositionen $\left(|\alpha|=3,1^{\circ}\right)$ um knapp $50 \%$ ab (Abb. 3.4.8).

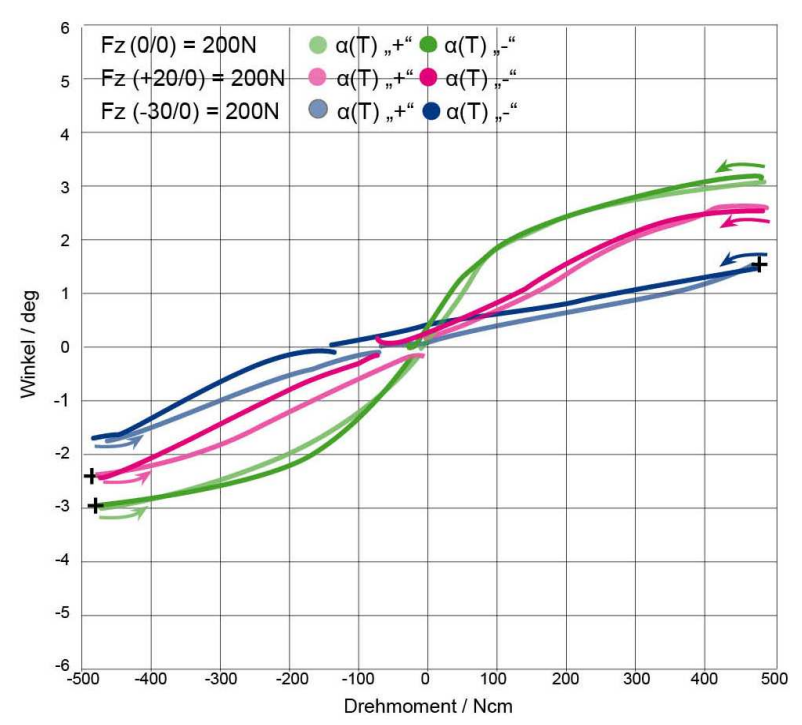

Abb. 3.4.8 Th 2/3 A: Drehmoment-DrehwinkelKennlinie bei verschiedenen Vorlastpositionen.

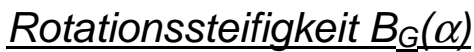

Die mittlere Steifigkeit $B_{G}(\alpha=0)$ und die Hysterese nehmen bei ventraler und dorsaler Vorlast zu. 
Sukzessive Resektion der Articulationes zygapophysiales von Th2/Th3 A

Die $\alpha(T)$-Kennlinie verändert sich merklich nach der sukzessiven Resektion der rechten, dann linken Wirbelbogengelenke. Nach der ersten Resektion nimmt der Bewegungsumfang geringfügig um $1,2^{\circ}$ ab. Diese Beobachtung gilt für alle betrachteten Vorlastpositionen. Nach Resektion des zweiten Art. zygapophysialis wird wieder eine symmetrische $\alpha(T)$ Abhängigkeit mit einem weit kleineren Rotationsbereich von $|\alpha|=3,9^{\circ}$ beobachtet (Abb. 3.4.9).

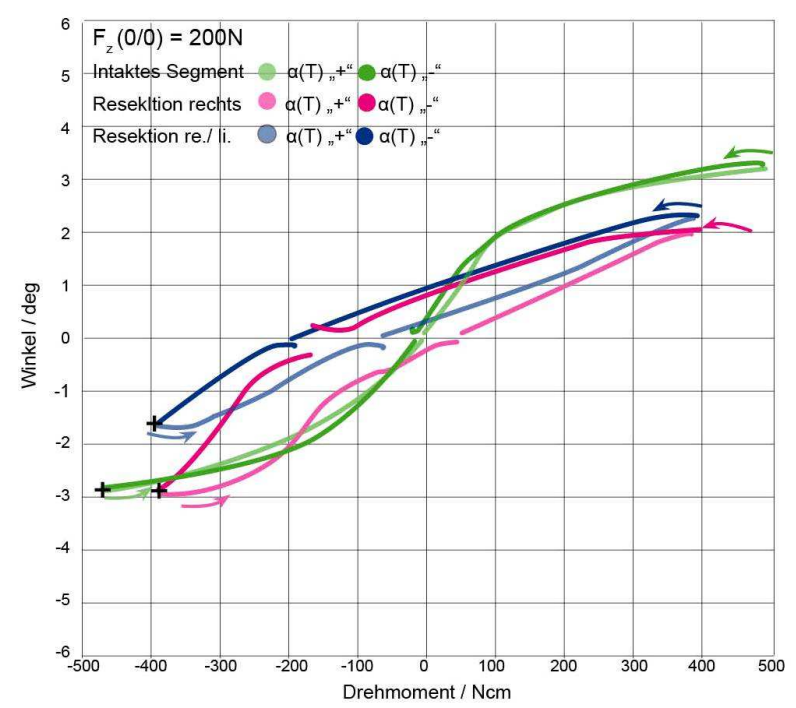

Abb. 3.4.9: Drehmoment-Drehwinkel-Kennlinie nach Resektion der Gelenke. Lateralflexion; grün: intaktes Segment, pink: nach Resektion des rechten Gelenks, blau: nach beidseitiger Resektion.

Die differenzielle Steifigkeit nimmt bei der Resektion der rechten und danach der linken Art. zygapophysialis zu (Abb. 3.4.10). Das Bewegungssegment versteift immer mehr.

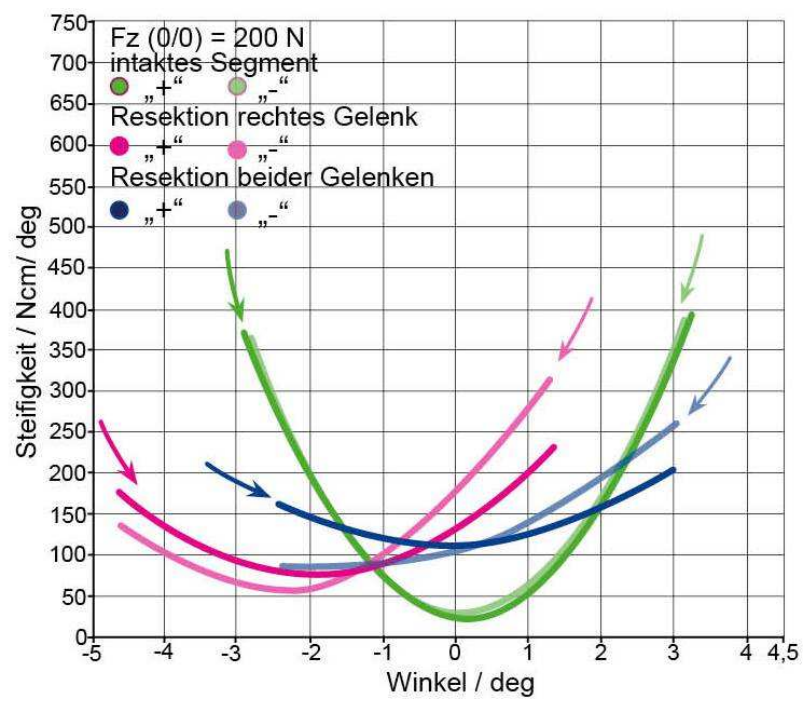

Abb. 3.4.10: Die Rotationssteifigkeit $B_{G}(\alpha)$ in $A b-$ hängigkeit des Drehwinkels. Lateralflexion; grün: intaktes Segment, pink: nach Resektion des rechten Gelenks, blau: nach beidseitiger Resektion. 


\section{Richtung der Schraubachse e $(\alpha)$}

Eine Verschiebung des Kraftvektors nach dorsal verändert das Verhalten des Richtungsvektors der IHA(Tx). In der Lateralflexion nach links (positive Drehung) steigt die ey-Komponente an. Die Schraubachse schwenkt in der Frontalebene nach links und für Rechtsneigung umgekehrt nach rechts. Bei maximaler Linksneigung ist $e_{Y}=0,08$ und $e_{X}=0,99$, was einem Neigungswinkel von $85,4^{\circ}$ nach links lateral entspricht. Somit verkippt sich die momentane Schraubachse geringfügig um $4,6^{\circ}$ von der senkrechten Stellung nach lin ks. In der maximaler Rechtsneigung beträgt die Verkippung nach rechts $10,4^{\circ}$ vo $\mathrm{n}$ der senkrechten Stellung der IHA. Das Verhalten der $\mathrm{e}_{\mathrm{z}}$-Komponente entspricht einer gleichmäßigen Bewegung des oberen Wirbels entlang der z-Achse: Aus maximaler Rechtsneigung von unten nach oben und wieder nach unten, aus maximaler Linksneigung wieder von unten nach oben (Abb. 3.4.11. A).

Bei ventraler Belastung ist der Verlauf der $e_{Y}$ Komponente umgekehrt (Abb. 3.4.11 B). Während einer Rechtsneigung kommt es zu einer lateralen Verkippung von rechts nach links um $8,2^{\circ}$ und in der Linksneigung u $\mathrm{m} 11,4^{\circ}$ nach rechts.

Im gesamten Messzyklus wandert die IHA geringfügig nach kranial.
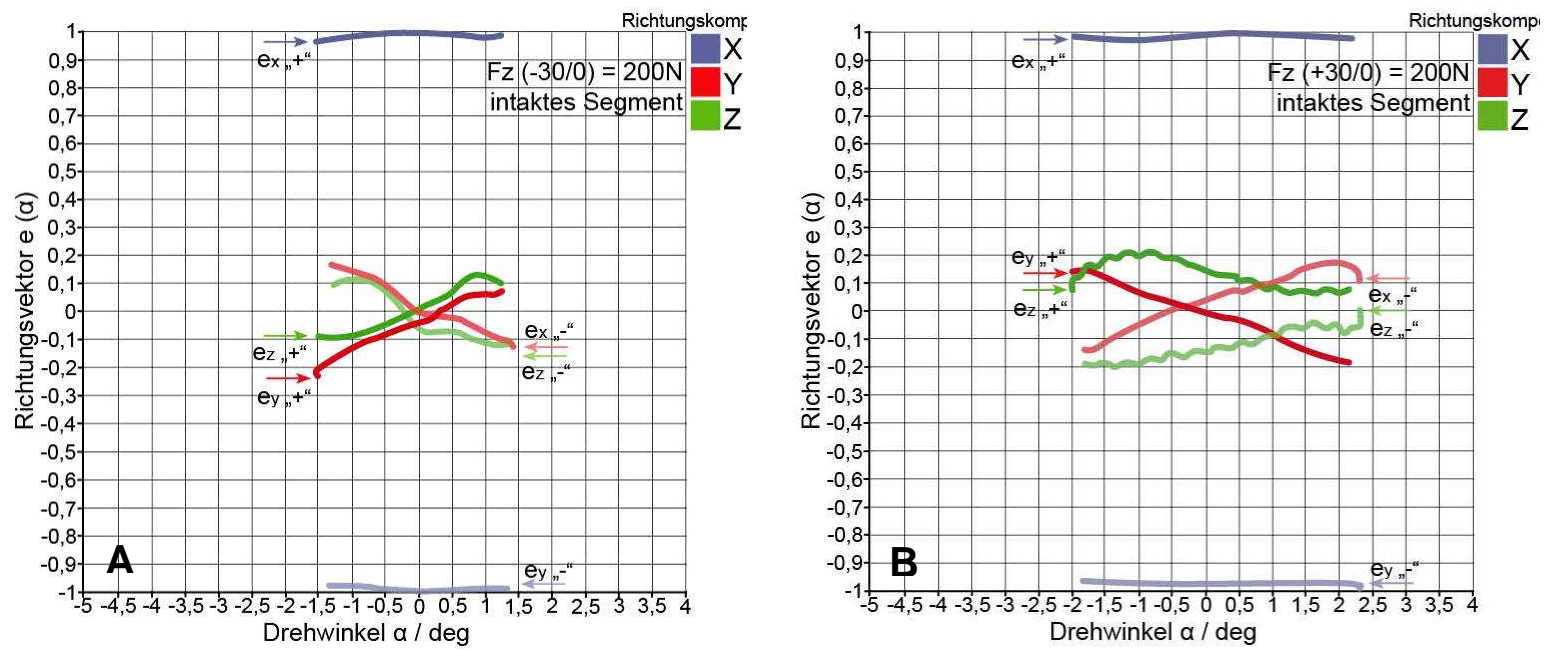

Abb. 3.4.11 A/B: Die Komponenten des Richtungsvektors von Th $2 / 3 \mathrm{~A}$ bei verschiedenen Vorlastpositionen $\mathrm{F}_{\mathrm{Z}}=200 \mathrm{~N}(\mathbf{A})$ dorsal $(-30 / 0)(B)$ ventral $(+30 / 0)$. 


\section{Schraubsteigung $\tau(\alpha)$}

Die momentane Schraubsteigung eines Messzyklus nimmt in Abhängigkeit des Drehwinkels zu. Die Veränderungen sind gering. Die Zunahme ist bei dorsaler Vorlastposition größer.

\section{Sukzessive Resektion der Articulationes zygapophysiales von Th2/Th3 A}

Der Anstieg der y- und z-Komponente nach Resektion des rechten Bogengelenks ist nur einseitig und asymmetrisch. Für positive Drehwinkel $\alpha$ sind alle drei Richtungskomponente parallel zueinander und zum angreifenden Drehmoment $T_{x}$. Für negative Drehwinkel $\alpha$ (Rechtsneigung) verkippen die momentanen Schraubachsen zunehmend nach rechts. Die Neigung der Schraubachse gegen die Frontalebene beträgt $6,3^{\circ}$ von der senkrechter Stellung der IHA (Abb. 3.4.12 A).

Die Entfernung des zweiten Bogengelenks hebt die Asymmetrie wieder auf. Die momentanen Schraubachsen verkippen zur Seite der jeweiligen Neigungsrichtung. In maximaler Rechtsneigung $\left(\alpha<0^{\circ}\right)$ verkippt die IHA um $12,6^{\circ}$ nach rechts und in maximaler Linksneigung $\left(\alpha>0^{\circ}\right)$ bis um $14,9^{\circ}$ nach links (Abb. 3.4.12 B).
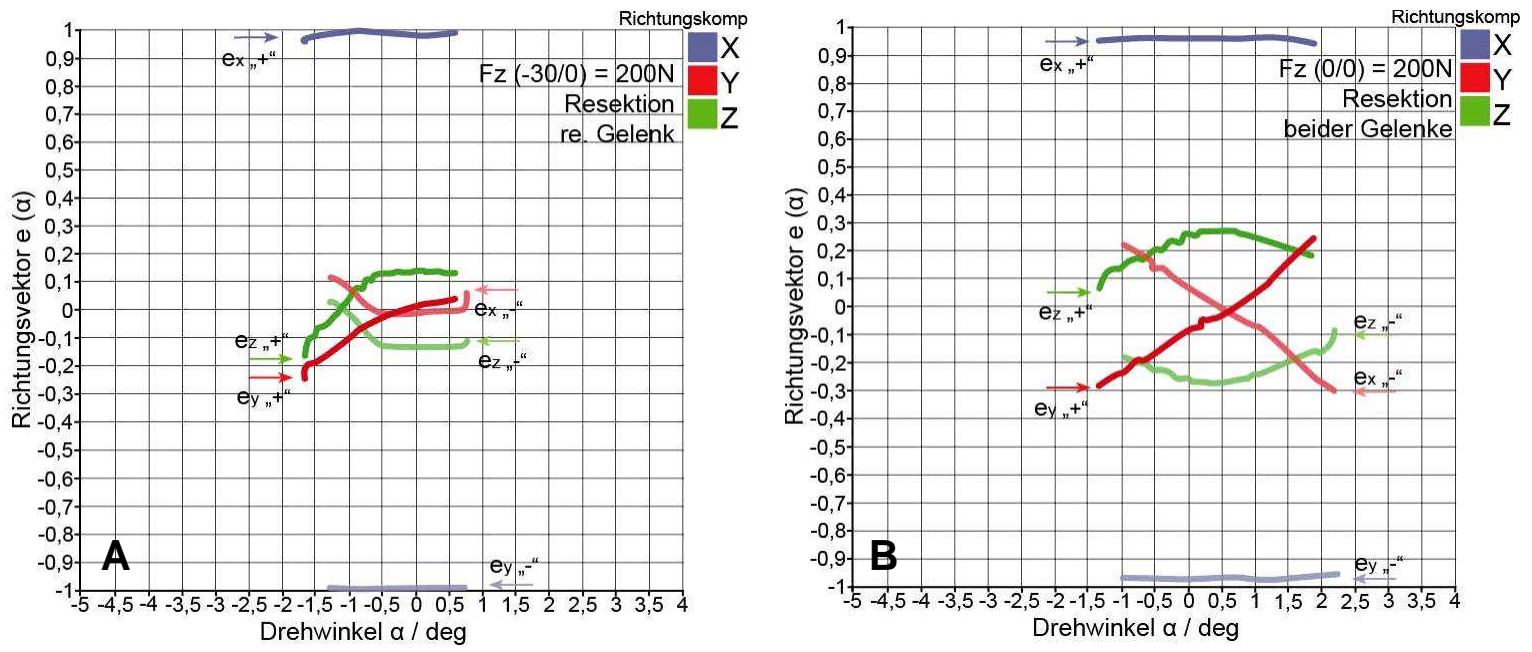

Abb. 3.4.12 A/B: Die Komponenten des Richtungsvektors von Th 2/3 A nach Resektion des rechten Bogengelenks $\mathrm{F}_{Z}(-30 / 0)(\mathbf{A})$ und des zweiten Gelenks $\mathrm{F}_{Z}(0 / 0)(\mathbf{B})$. 
Nach der Entfernung eines Gelenks ist der Verlauf der Schraubsteigung $\tau(\alpha)$ asymmetrisch. Für eine Rotation in negativer Richtung (Rechtsneigung) ist die Schraubsteigung größer als für positive Drehwinkel (Linksneigung). Nach beidseitiger Resektion der Artt. zygapophysiales kommt es näherungsweise zu einer ebenen Bewegung.

\subsubsection{Verschiebung der Vorlast entlang der y-Achse;}

$$
I(t)=\left(0, I_{Y}(t), 0\right) \wedge F=(0,0,200 N)
$$

Die Verschiebung der axialen Vorlast von $\mathrm{F}_{\mathrm{Z}}=200 \mathrm{~N}$ in der Transversallebene verändert die Position der Schraubachsen unterhalb der Bandscheibe wesentlich. Für laterale Vorlasten verschiebt sich die IHA um 12-16mm zur Seite der jeweiligen Belastung (Abb. 3. 4.13 A/B). Für eine Rotation in beide Drehrichtungen wandern die momentanen Schraubachsen von oben nach unten zur Gegenseite, auf einer Bahn unterhalb der Bandscheibe des darunter liegenden Th3/4- Bewegungssegmentes.
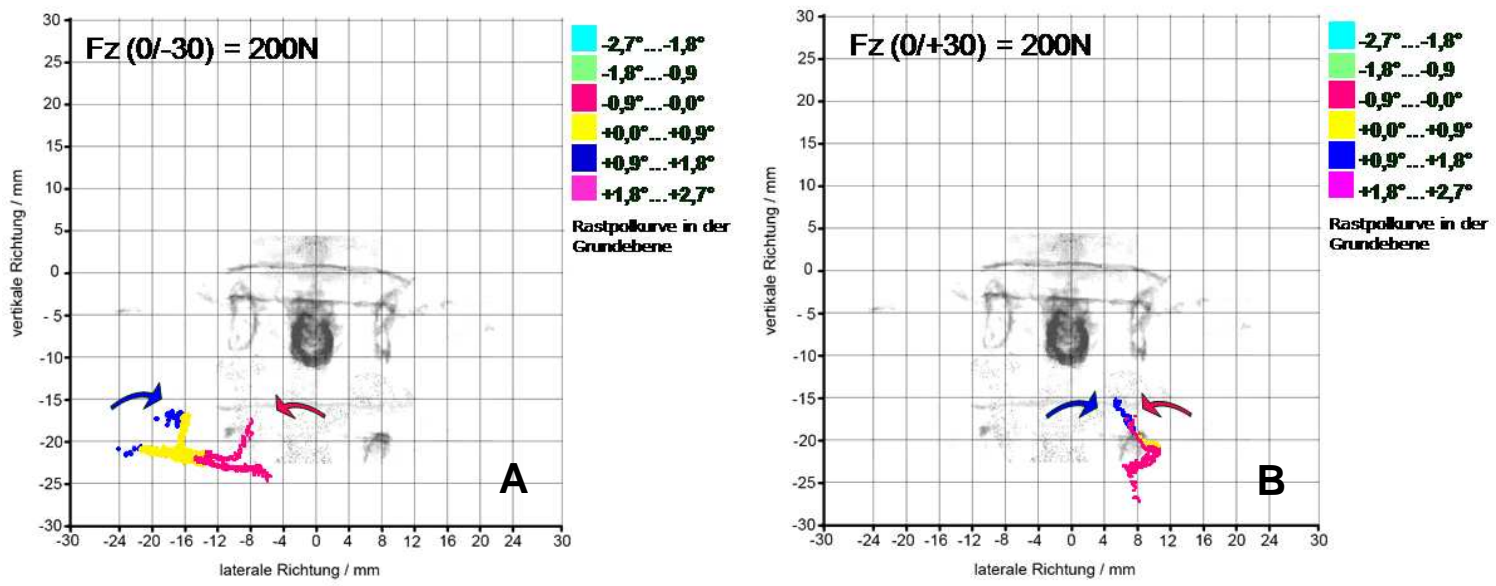

Abb. 3.4.13 A/B: Durchstoßpunkte $R_{i}(\alpha)$ der momentanen Schraubachsen für eine Neigung in positiver und negativer Richtung bei einer axialen Vorlast rechts $F_{Z}(0 /-30)=200 \mathrm{~N}(\mathbf{A})$, und links $F_{Z}(0 /+30)=200 N(B)$. Ansicht von ventral. 
Sukzessive Resektion der Articulationes zygapophysiales Th2/Th3 A

Nach Resektion des rechten Art. zygapophysiales resultiert eine stark komprimierte und nach kranial offene, v-förmige Rastpolkurve. Die Verkippung des oberen Wirbelkörpers zur rechten Seite führt zu einer Wanderung der IHAs auf der Höhe des resezierten Gelenks und liegt somit knapp unter der Bandscheibe des Th 2/3Segments. Die IHA verschiebt sich um $10 \mathrm{~mm}$ nach kranial und verschmälert sich in der Sagittalen fast um die Hälfte auf 8mm (Abb. 3.4.14 A). Bei Applikation einer links-lateralen Vorlast kommt es zur einer geringen Wanderung der Rastpolkurve nach kranial $(\Delta \mathrm{z}=5 \mathrm{~mm})$ auf der Seite des vorhandene Bogengelenkes. Die IHA liegt auf der Höhe der Zwischenwirbelscheibe des Th 3/4-Segments (Abb. 3.4.14 B).
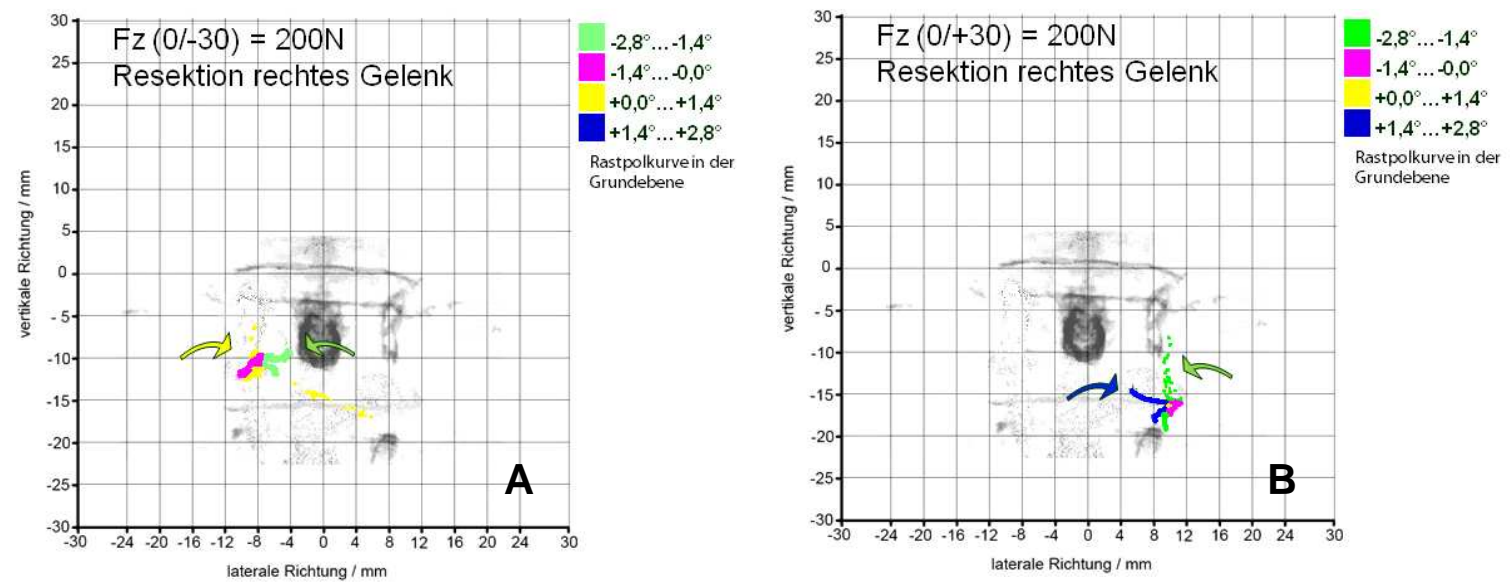

Abb. 3.4.14 A/B: Durchstoßpunkte $R_{i}(\alpha)$ der momentanen Schraubachsen für eine Neigung in positiver und negativer Richtung nach Resektion des rechten Art. zygapophysiales bei einer axialen Vorlast rechts $F_{Z}(0 /-30)=200 \mathrm{~N}(A)$, bzw. links $F_{Z}(0 /+30)=200 N(B)$.

Die beidseitige Resektion von Artt. zygapophysiales verändert das Verhalten der IHAs für alle gemessenen lateralen Vorlastpositionen unwesentlich. Rastpolkurven für Rechts- und Linksneigung fallen praktisch zusammen.

Die Drehmoment-Drehwinkel-Kennlinien zeigen einen weniger ausgeprägten Verlauf. Der Bewegungsumfang ist für die rechte Vorlastposition mit $|\alpha|=2$ etwas kleiner als für die linke mit $|\alpha|=3\left(F_{Z}(0 /+30)\right)$. 


\subsubsection{Bewegungssegment Th 2/3 B}

\subsubsection{Applikation eines Drehmoments ohne/ mit axialer Vorlast;}

$$
\underline{I}(\mathrm{t})=\left(\underline{I}_{x}(\mathrm{t}), 0,0\right) \wedge \mathrm{F}_{\mathrm{Z}}=(0,0,0)
$$

\section{Rastpolkurven}

Die Rastpolkurven $r_{i}(\alpha)$ des Bewegungssegments Th 2/3 B zeigen keine nennenswerten Unterschiede zu dem Verlauf der Rastpolkurven des Th 2/3 ASegments (Kapitel 3.4.1, S. 140).
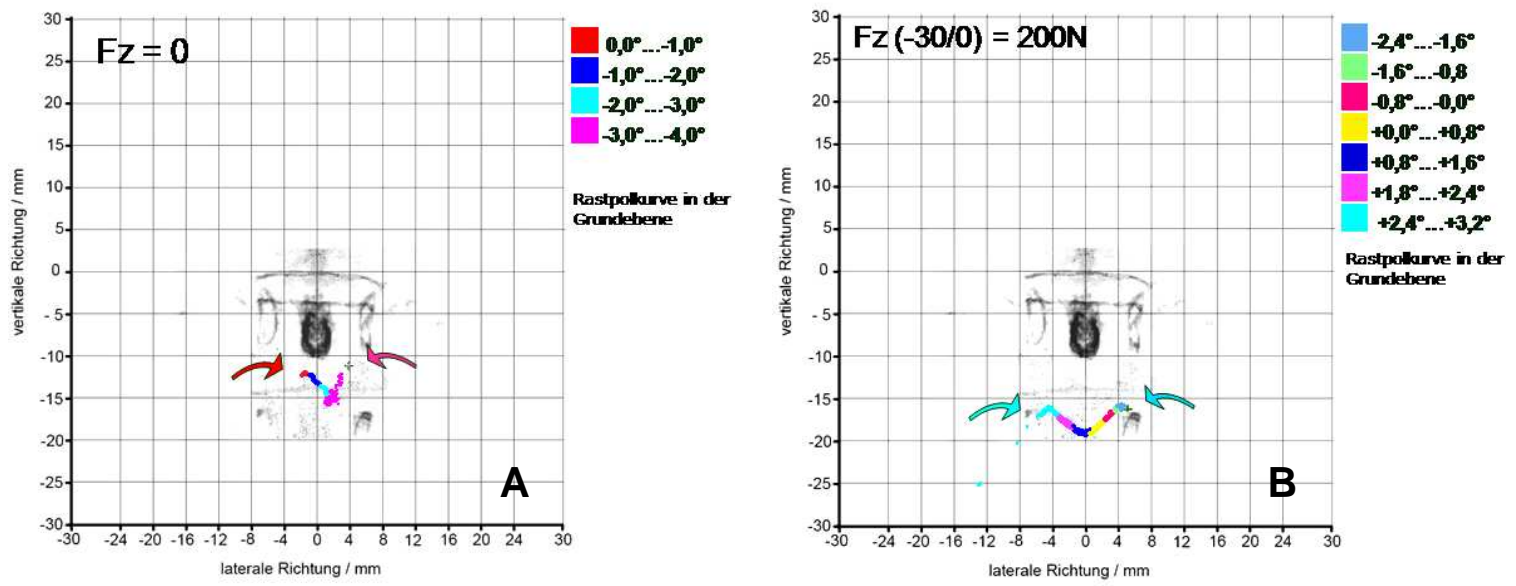

Abb. 3.4.15 A/B: Durchstoßpunkte $\mathrm{R}_{\mathrm{i}}(\alpha)$ der momentanen Schraubachsen für eine Drehung in positiver und negativer Drehrichtung ohne axiale Vorlast $F_{Z}(0 / 0)=0 \mathrm{~N}(A)$ und für dorsale Vorlastposition $\mathrm{F}_{Z}(-30 / 0)=200 \mathrm{~N}(\mathrm{~B})$. Ansicht von ventral.

Die Rastpolkurven des zweiten Bewegungssegments Th 2/3 B verlaufen um $9 \mathrm{~mm}$ weiter nach kranial versetzt als die am Th 2/3 A gemessenen IHAs. Sie liegen auf der Höhe der Zwischenwirbelscheibe des Th3/4-Segments. Ihr Wanderbereich ist viel kleiner, was auch den anatomischen Gegebenheiten des Th 2/3 B Bewegungssegments entspricht. Die Bewegung ist eben und gleichmäßig. Die Schraubachsen sind nicht ortsfest (Abb. 3.4.15 A/B). Diese Beobachtung gilt für alle gemessene Vorlastpositionen und Vorlastgrößen. 


\section{Drehwinkel- Drehmoment- Kennlinie}

Die Abbildung 3.4.16 zeigt die gemessenen Drehwinkel-Drehmoment-Kennlinien bei Applikation eines Drehmoments $T_{X}$ ohne axiale Vorlast. Die $\alpha(T)$-Funktion ist deutlich sigmoid geformt. Die Hysterese $\Delta \alpha(T=0)$ ist klein und beträgt für $T=0$ $\alpha=0,45^{\circ}$. Der Bewegungsumfang (ROM) beträgt $6,5^{\circ}$ mit angrei fendem Drehmoment von maximal $400 \mathrm{~N} / \mathrm{cm}$.

Bei Verschiebung der axialen Vorlast in sagittaler Richtung verkleinert sich der Bewegungsumfang $(\mathrm{ROM})$ und beträgt sowohl für ventrale als auch für dorsale Vorlastpositionen $|\alpha|=5,4^{\circ}$ (Abb. 3.4.17).

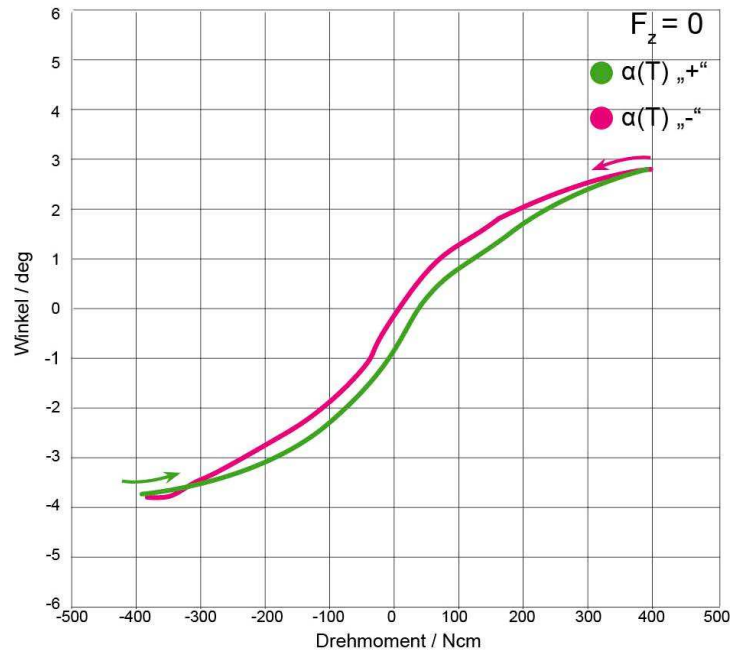

Abb. 3.4.16: Drehmoment-Drehwinkel-Kennlinie Lateralflexion; ohne axiale Vorlast.

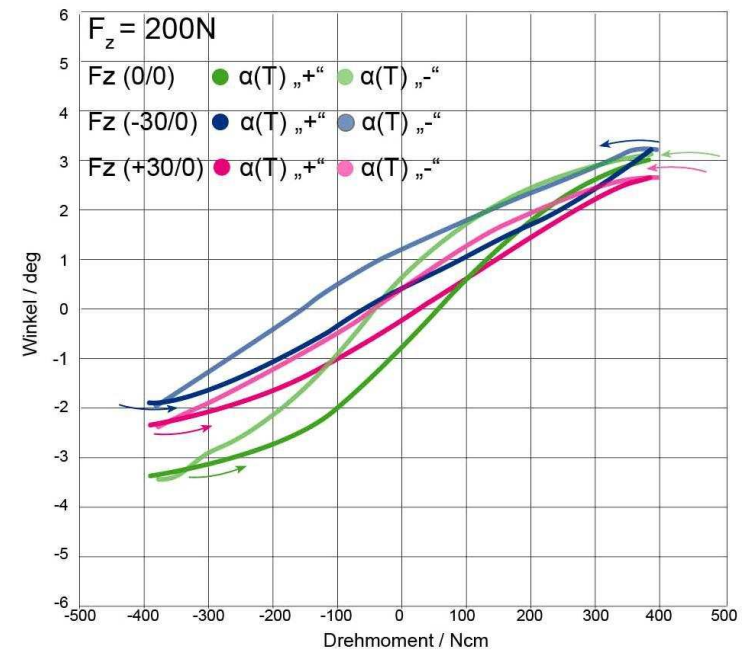

Abb. 3.4.17: Drehmoment-Drehwinkel-Kennlinie Lateralflexion; grün: zentrale Vorlastposition, pink: ventrale Vorlastposition $\mathrm{F}_{\mathrm{Z}}(+30 / 0)$, blau: dorsale Vorlastposition $\mathrm{F}_{Z}(-30 / 0)$. 


\section{$\underline{\text { Rotationssteifigkeit } \underline{B}_{G}(\alpha)}$}

Die minimale Steifigkeit $B(\alpha)$ liegt bei $50 \mathrm{Ncm} /{ }^{\circ}$. In maximaler Rechtsneigung so wie in der Linksneigung (positive Drehwinkel $\alpha$ ) steigt die Steifigkeit $B(\alpha)$ bis zu einem Maximum von $250 \%$ Ncm an (Abb. 3.4.18).

Die mittlere Steifigkeit $B_{G}(\alpha=0)$ nimmt bei ventraler und dorsaler Vorlast leicht zu (Abb. 3.4.19).

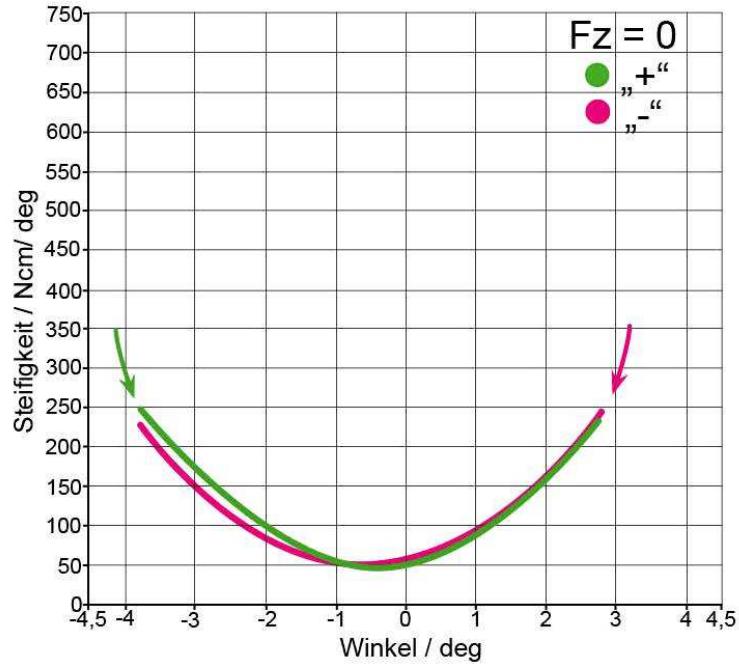

Abb.3.4.18: Die Rotationssteifigkeit $\mathrm{B}_{\mathrm{G}}(\alpha)$ in Abhängigkeit des Drehwinkels. Lateralflexion; ohne axiale Vorlast.

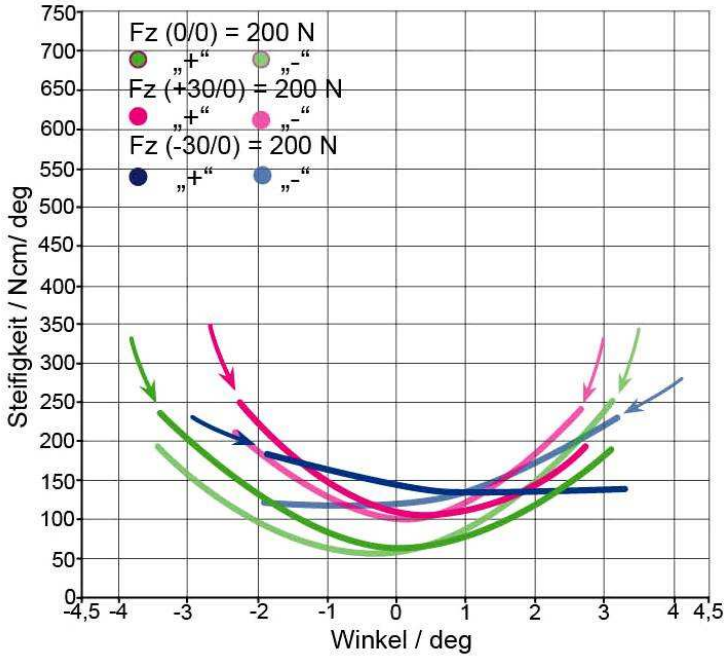

Abb.3.4.19: Die Rotationssteifigkeit $B_{G}(\alpha)$ in Abhängigkeit des Drehwinkels. Lateralflexion; grün: $F z(0 / 0)$, pink: $F_{Z}(+30 / 0)$, blau: $F_{Z}(-30 / 0)$.

\section{$\underline{\text { Richtung der Schraubachse e }(\alpha)}$}

Die $x$-Komponente ist $e_{x}(\alpha) \cong 1$, somit sind die IHAs parallel zum Drehmoment $T_{x}$ ausgerichtet. Die momentanen Schraubachsen verkippen zur Seite der jeweiligen Neigungsrichtung. Die laterale Verkippung der Schraubachsen beträgt in maximaler Rechtsneigung $1,14^{\circ}$ nach rechts und in maximaler Linksneigung $2,3^{\circ}$ nach links-lateral (Abb. 3.4.20).

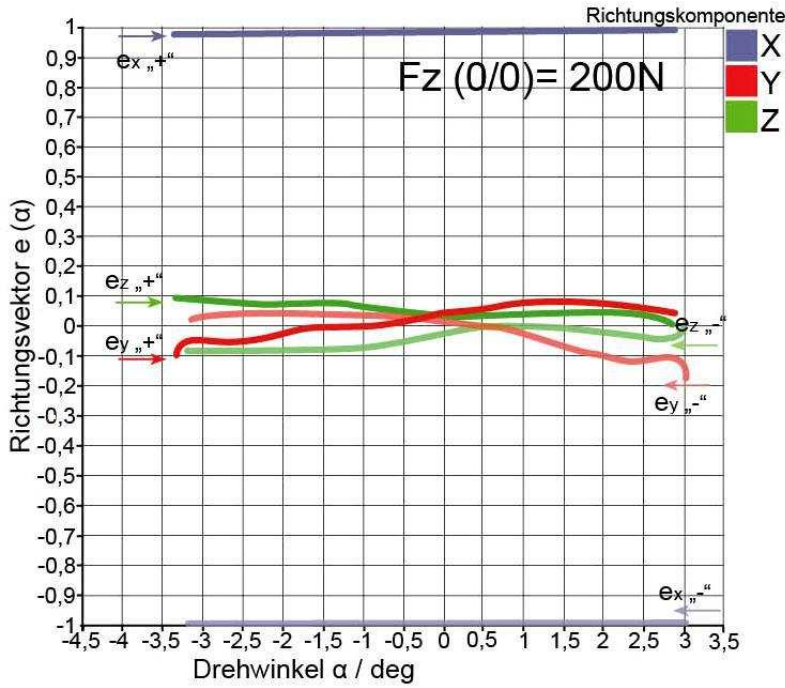

Abb. 3.4.20: Die Komponenten des Richtungsvektors $\underline{e}$ (Th2/3 B) in Abhängigkeit des Drehwinkels $\alpha$ für einen kompletten Messzyklus. 
Es handelte sich um eine ebene Bewegung. In der Lateralflexion beträgt die momentane Schraubsteigung $\tau(\alpha) \approx 0,1 \mathrm{~mm} / \mathrm{Grad}(\mathrm{Abb}$. 3.4.21).

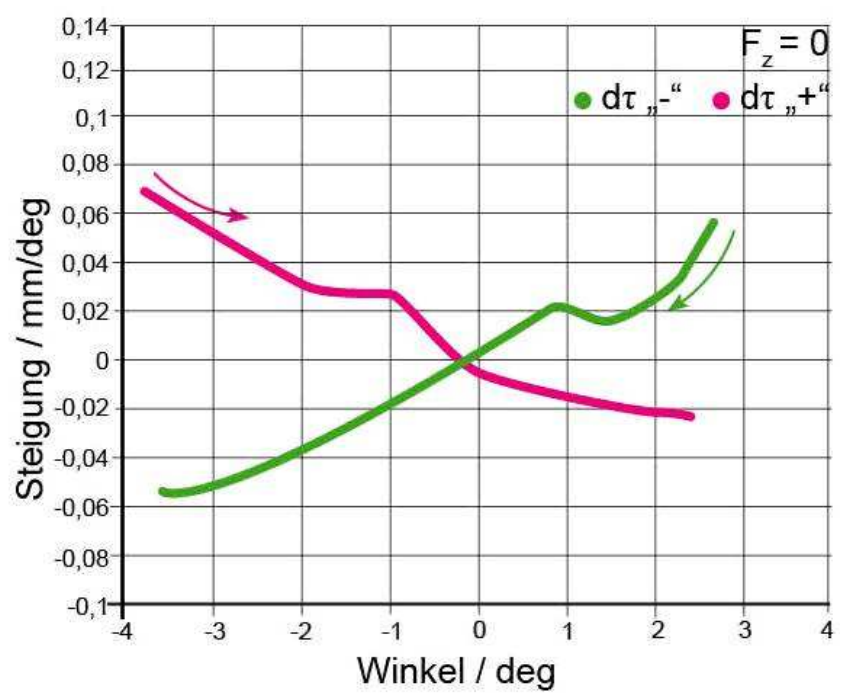

Abb. 3.4.21: Die momentane Schraubsteigung $\tau(\alpha)$ in Abhängigkeit des Drehwinkels $\alpha$.

Für dorsale Vorlastpositionen schwenkt die Schraubachse bei der Linksneigung in der Frontalebene nach links und bei Rechtsneigung umgekehrt nach rechts. Der Verkippungswinkel der IHAs von der senkrechten Stellung beträgt bei maximaler Linksneigung nach links $76,6^{\circ}$ und bei maximaler Rec htsneigung nach rechts $8,1^{\circ}$ (Abb. 3.4.22 A).
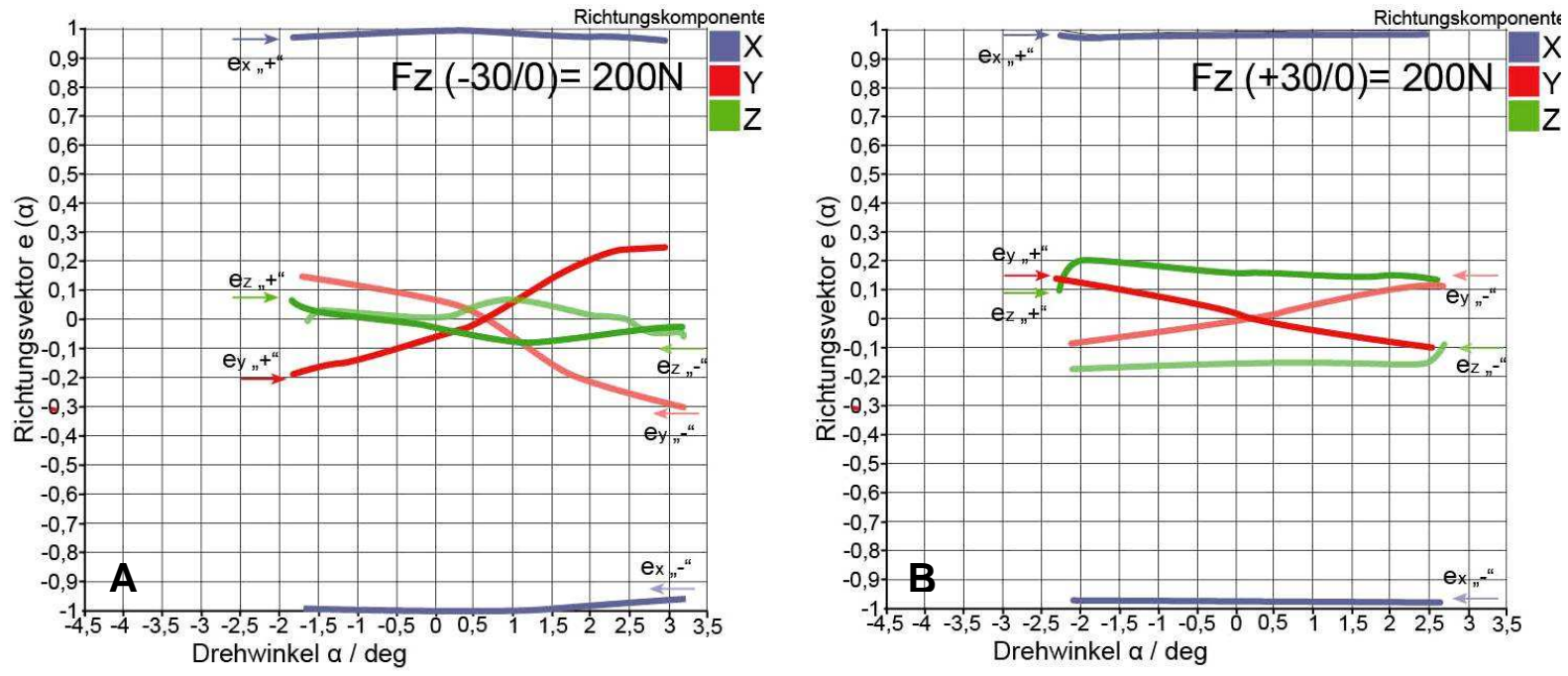

Abb. 3.4.22 A/B : Die Komponenten des Richtungsvektors von Th $2 / 3 \mathrm{~B}$ bei verschiedenen Vorlastpositionen $\mathrm{F}_{Z}=200 \mathrm{~N}(\mathbf{A})$ dorsal $(-30 / 0)(B)$ ventral $(+30 / 0)$. 
Bei ventraler Belastung ist der Verlauf der ey Komponente umgekehrt (Abb. 3.4.22. B). Der Verkippungswinkel der IHAs von der senkrechten Stellung ist sowohl bei maximaler Linksneigung nach links als auch bei maximaler Rechtsneigung nach rechts gleich $84,2^{\circ}$. Das Verhalten der $\mathrm{e}_{\mathrm{z}}$-Komponente entspricht einer gleichmäßigen Bewegung des oberen Wirbels entlang der z-Achse. Bei einem ventralen Kraftangriff entfernen sich die Wirbelkörper leicht voneinander, die Bandscheibe elongiert (Abb. 3.4. 22. A und B).

Die momentane Schraubsteigung eines Messzyklus nimmt in Abhängigkeit des Drehwinkels geringfügig zu.

\subsubsection{Verschiebung der Vorlast entlang der y-Achse;}

$$
I(t)=\left(0, I_{Y}(t), 0\right) \wedge F=(0,0,200 N)
$$

Bei einer Verschiebung der axialen Vorlast von $F_{Z}=200 \mathrm{~N}$ in der Transversallebene gleicht das Verhalten der Rastpolkurven $r(\alpha)$ des Bewegungssegments Th 2/3 B prinzipiell dem Verlauf der Rastpolkurven des Th 2/3 A-Segments (Kapitel 3.4.1.4, S. 148). Der Unterschied liegt im reduzierten Wanderbereich der IHAs, was wiederrum die anatomischen Gegebenheiten des Th 2/3 B Bewegungssegments widerspiegelt. Die Bewegung ist eben und gleichmäßig (Abb. 3.4.23 A/B). Diese Beobachtung gilt für alle gemessenen Vorlastpositionen und Vorlastgrößen.
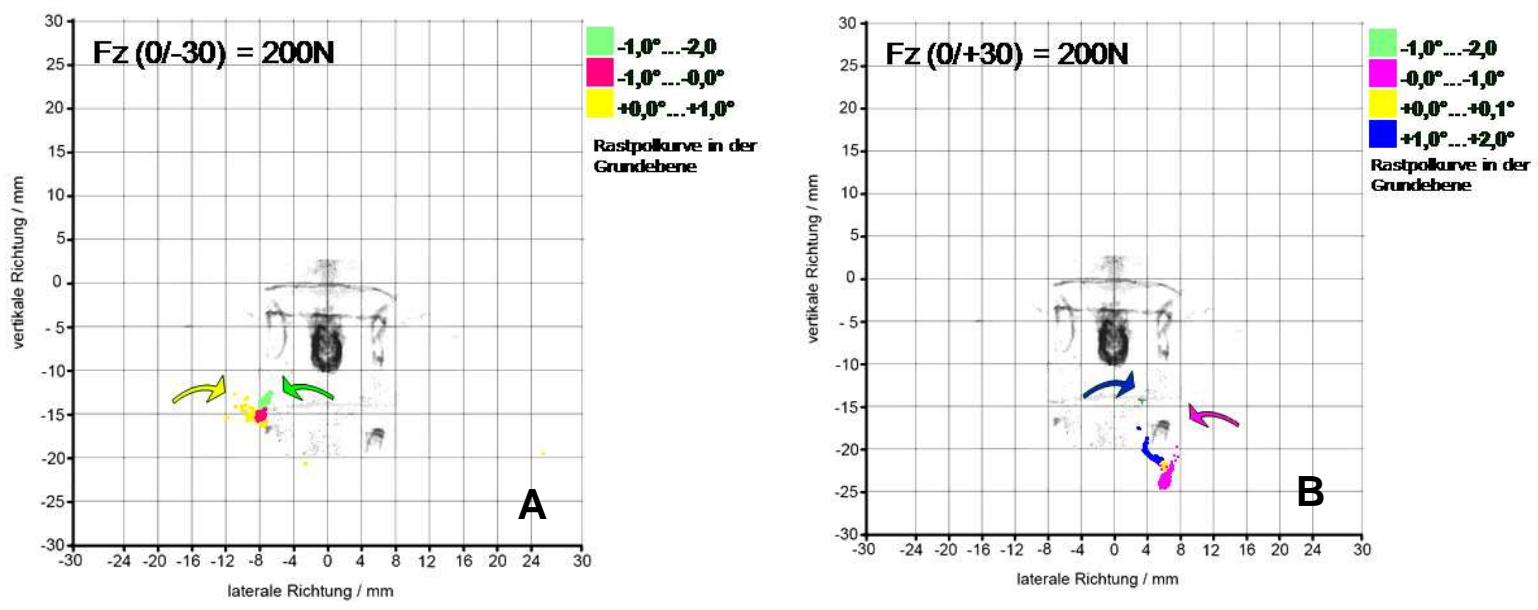

Abb. 3.4.23 Th 2/3 B: Durchstoßpunkte $\mathrm{R}_{\mathrm{i}}(\alpha)$ der momentanen Schraubachsen für eine Drehung in positiver und negativer Drehrichtung bei einer lateralen Vorlast rechts $F_{Z}(0 /-30)=200 \mathrm{~N}$ (A), und links $F_{Z}(0 /+30)=200 N(B)$. Ansicht von ventral. 
Die Drehmoment-Drehwinkel-Kennlinien zeigen einen weniger ausgeprägten Verlauf (Abb. 3.4.24). Beide $\alpha(T)$-Funktionen sind zueinander vertikal und horizontal spiegelsymmetrisch. Der Bewegungsumfang ist für die rechte Vorlastposition mit $|\alpha|=2,5^{\circ}$ etwas kleiner als für die linke mit $|\alpha|=3^{\circ}\left(F_{z}(0 /+30)\right)$.

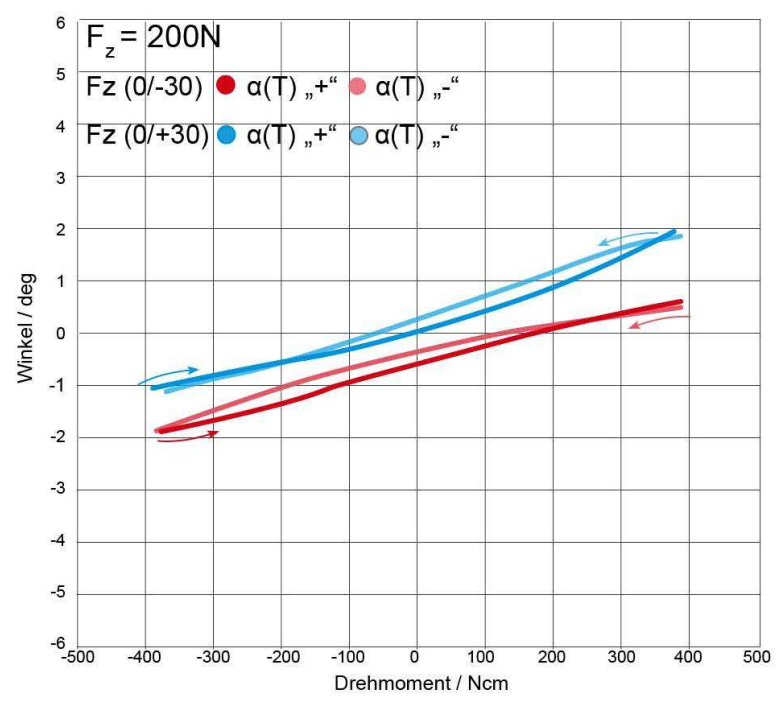

Abb. 3.4.24: Drehmoment-Drehwinkel-Kennlinien von Th 2/3 B. Lateralflexion.

Bei einer Vorlastposition rechts-lateral ist die minimale Steifigkeit höher als bei einer links-lateralen und beträgt $\approx 275 \%$ Ncm. Das Bewegungssegment ist in der Lateralflexion bei einer rechtsseitigen Belastung steifer als bei einer linksseitigen Belastung (Abb. 3.4.25).

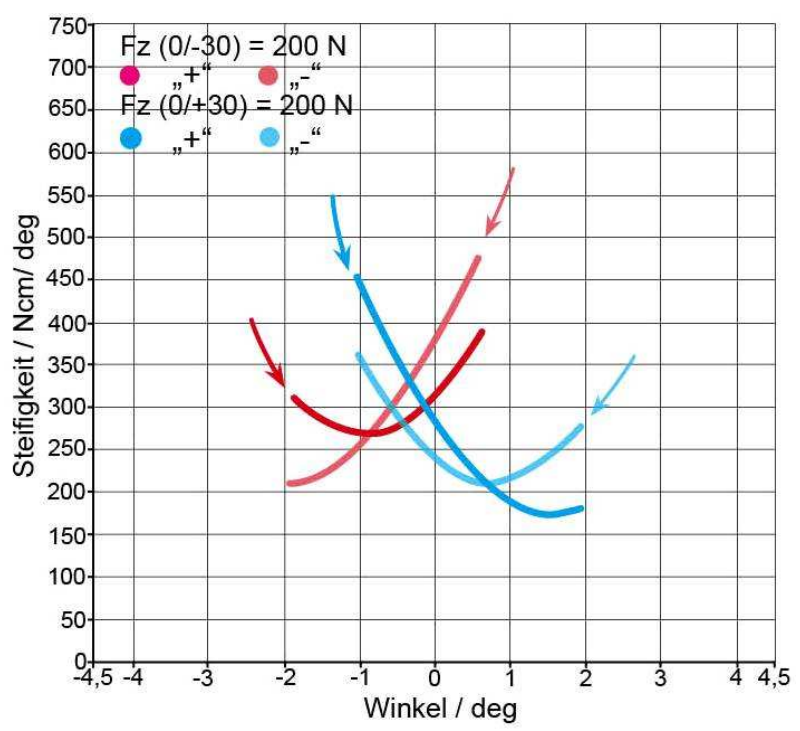

Abb. 3.4.25: Steifigkeit $B_{G}(\alpha)$ von Th $2 / 3 B$. Lateralflexion. 
Der Anstieg der y-Komponente zur Seite der Belastung ist nur einseitig und asymmetrisch. Bei einer rechts-lateralen Belastung sind die momentanen Schraubachsen sowohl für die Rechts,- als auch für die Linksneigung nach rechts lateral geneigt. In maximaler Rechtsneigung verkippt die momentane Schraubachse um $13,3^{\circ}$ und in maximaler Linksneigung um $4,6^{\circ}$ nach rechts lateral (Abb. 3.4.26 A). Für die links-laterale Vorlast verkippen die IHAs in einem kompletten Messzyklus nach links lateral, allerdings mit einer schwächeren Ausprägung als bei einer rechts-lateralen Belastung (Abb. 3.4.26 B).
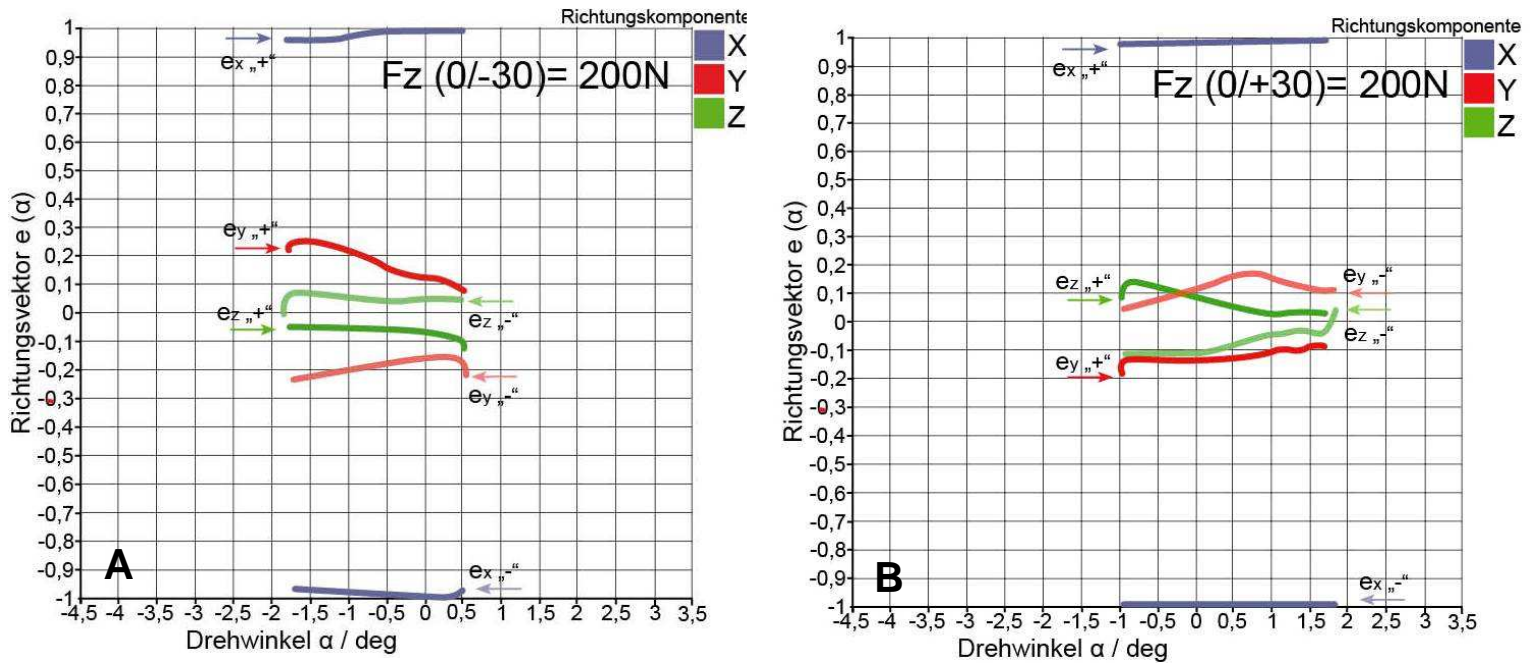

Abb. 3.4.26 A/B: Die Komponenten des Richtungsvektors von Th $2 / 3 \mathrm{~B}$ in der Lateralflexion. Vorlastposition rechts-lateral $F_{Z}(0 /-30)=200 N(A)$ und links-lateral $F_{Z}(0 /+30)(B)$. 


\subsubsection{Vergleich intakter Bewegungssegmente Th 2/3 A und Th 2/3 B:}

\begin{tabular}{|c|c|c|}
\hline Merkmal & intaktes Segment Th 2/3 A & intaktes Segment Th 2/3 B \\
\hline \multicolumn{3}{|c|}{ Verhalten der IHAs bei Änderung der Vorlastposition } \\
\hline dorsale Vorlast & $\begin{array}{l}\text { IHAs liegen kaudal und wandern leicht } \\
\text { nach kranial und von einem WBG in das } \\
\text { andere auf der Höhe des Th } 4 . \\
\text { IHAs parallel zu Tx. } \\
\text { max. Lateralflexion: } \\
\text { Verkippen nach lateral zur Seite der } \\
\text { jeweiligen Neigungsrichtung. }\end{array}$ & $\begin{array}{l}\text { IHAs liegen kaudal und wandern } \\
\text { nach kranial und von einem WBG in } \\
\text { das andere auf der Höhe der Band- } \\
\text { scheibe Th } 3 / 4 \text {. } \\
\text { IHAs parallel zu } T_{x} \text {. } \\
\text { max. Lateralflexion: } \\
\text { Verkippen nach lateral zur Seite der } \\
\text { jeweiligen Neigungsrichtung. }\end{array}$ \\
\hline ventrale Vorlast & $\begin{array}{l}\text { IHAs v-förmig, geringe IHA-Wanderung. } \\
\text { IHAs parallel zu } T_{x} \text {. } \\
\text { max. Lateralflexion: } \\
\text { Verkippen nach lateral zur Seite der } \\
\text { jeweiligen Neigungsrichtung. }\end{array}$ & $\begin{array}{l}\text { IHAs verschiebt sich leicht nach } \\
\text { kranial. } \\
\text { IHAs parallel zu } T_{x} \text {. } \\
\text { max. Lateralflexion: } \\
\text { Verkippen nach lateral zur Seite der } \\
\text { jeweiligen Neigungsrichtung. }\end{array}$ \\
\hline laterale Vorlast & $\begin{array}{l}\text { IHAs liegen kaudal auf der Seite der } \\
\text { jeweiligen Neigungsrichung. }\end{array}$ & $\begin{array}{l}\text { IHAs liegen kaudal auf der Seite der } \\
\text { jeweiligen Neigungsrichung. }\end{array}$ \\
\hline \multicolumn{3}{|c|}{ Verhalten von ROM und Steifigkeit } \\
\hline dorsale Vorlast & $\begin{array}{l}\text { ROM } \downarrow \\
\text { Hysterese } \downarrow \\
\text { minimale Steifigkeit } \uparrow \\
\end{array}$ & $\begin{array}{l}\text { ROM } \downarrow \\
\text { Hysterese } \downarrow \\
\text { minimale Steifigkeit } \uparrow\end{array}$ \\
\hline ventrale Vorlast & $\begin{array}{l}\text { ROM } \downarrow \\
\text { Hysterese } \downarrow \\
\text { minimale Steifigkeit } \uparrow \text { geringfügig }\end{array}$ & $\begin{array}{l}\text { ROM } \downarrow \\
\text { Hysterese } \downarrow \\
\text { minimale Steifigkeit } \uparrow \text { geringfügig }\end{array}$ \\
\hline laterale Vorlast & $\begin{array}{l}\text { ROM } \downarrow \\
\text { minimale Steifigkeit } \downarrow\end{array}$ & $\begin{array}{l}\text { ROM } \downarrow \\
\text { minimale Steifigkeit } \downarrow\end{array}$ \\
\hline
\end{tabular}

Tab.3.7: Zusammenfassung der Ergebnisse der Versuchsreihe „Lateralflexion“ für Th 2/3 A und

Th 2/3 B. 


\subsubsection{Vergleich intaktes Bewegungssegment und Segment nach der Resektion der rechten Art. zygapophysialis (Th 2/3 A)}

\begin{tabular}{|c|c|c|}
\hline Merkmal & intaktes Segment Th 2/3 A & $\begin{array}{l}\text { Resektion rechten Art. zygapo- } \\
\text { physialis }\end{array}$ \\
\hline \multicolumn{3}{|c|}{ Verhalten der IHAs bei Änderung der Vorlastposition } \\
\hline dorsale Vorlast & $\begin{array}{l}\text { IHAs liegen kaudal und wandern leicht } \\
\text { nach kranial und von einem WBG in das } \\
\text { andere, auf der Höhe des Th } 4 . \\
\text { IHAs parallel zu TX. } \\
\text { max. Lateralflexion: } \\
\text { Verkippen nach lateral zur Seite der } \\
\text { jeweiligen Neigungsrichtung. }\end{array}$ & $\begin{array}{l}\text { IHAs nach kranial verlagert, auf der } \\
\text { Höhe der Bandscheibe Th } 3 / 4 \text {. } \\
\text { IHAs parallel zu } T_{x} \text {. } \\
\text { max. Lateralflexion: } \\
\text { Verkippen nach lateral zur Seite des } \\
\text { verbliebenes Gelenks. }\end{array}$ \\
\hline ventrale Vorlast & $\begin{array}{l}\text { IHAs v-förmig, geringe IHA-Wanderung. } \\
\text { IHAs parallel zu } T_{X} \text {. } \\
\text { max. Lateralflexion: } \\
\text { Verkippen nach lateral zur Seite der } \\
\text { jeweiligen Neigungsrichtung. }\end{array}$ & $\begin{array}{l}\text { IHAs verläuft in einem nach kranial } \\
\text { weit offenem Bogen auf der Höhe } \\
\text { der Bandscheibe Th } 3 / 4 \text {. } \\
\text { IHAs parallel zu TX. } \\
\text { max. Lateralflexion: } \\
\text { Verkippen nach lateral zur Seite des } \\
\text { verbliebenes Gelenks. }\end{array}$ \\
\hline laterale Vorlast & $\begin{array}{l}\text { IHAs liegen kaudal auf der Seite der } \\
\text { jeweiligen Neigungsrichung }\end{array}$ & $\begin{array}{l}\text { IHAs komprimiert und ortsfest } \\
\text { liegen weit kranial auf der Seite der } \\
\text { jeweiligen Neigungsrichung }\end{array}$ \\
\hline \multicolumn{3}{|c|}{ Verhalten von ROM und Steifigkeit } \\
\hline dorsale Vorlast & $\begin{array}{l}\text { ROM } \downarrow \\
\text { Hysterese } \downarrow \\
\text { minimale Steifigkeit } \uparrow \\
\end{array}$ & $\begin{array}{l}\mathrm{ROM} \uparrow \\
\text { Hysterese } \uparrow \\
\text { minimale Steifigkeit } \uparrow \\
\end{array}$ \\
\hline ventrale Vorlast & $\begin{array}{l}\text { ROM } \downarrow \\
\text { Hysterese } \downarrow \\
\text { minimale Steifigkeit } \uparrow \text { geringfügig }\end{array}$ & $\begin{array}{l}\mathrm{ROM} \uparrow \\
\text { Hysterese } \uparrow \\
\text { minimale Steifigkeit } \uparrow \text { geringfügig }\end{array}$ \\
\hline laterale Vorlast & $\begin{array}{l}\text { ROM } \downarrow \\
\text { Hysterese unverändert } \\
\text { minimale Steifigkeit } \downarrow \\
\end{array}$ & $\begin{array}{l}\mathrm{ROM} \downarrow \\
\text { Hysterese unverändert } \\
\text { minimale Steifigkeit } \downarrow\end{array}$ \\
\hline
\end{tabular}

Tab.3.8: Zusammenfassung der Ergebnisse der Versuchsreihe „Lateralflexion“ für Th 2/3 A nach Resektion der rechten Art. zygapophysialis. 


\subsubsection{Vergleich intaktes Bewegungssegment und} Bandscheibe- Bänder- Präparat (Th 2/3 A)

\begin{tabular}{|c|c|c|}
\hline Merkmal & intaktes Segment Th 2/3 A & Bandscheibe-Bänder-Präparat \\
\hline \multicolumn{3}{|c|}{ Verhalten der IHAs bei Änderung der Vorlastposition } \\
\hline dorsale Vorlast & $\begin{array}{l}\text { IHAs liegen kaudal und wandern leicht } \\
\text { nach kranial und von einem WBG in das } \\
\text { andere auf der Höhe des Th } 4 . \\
\text { IHAs parallel zu TX } \\
\text { max. Lateralflexion: } \\
\text { Verkippen nach lateral zur Seite der } \\
\text { jeweiligen Neigungsrichtung }\end{array}$ & $\begin{array}{l}\text { IHAs nach kranial verlagert auf der } \\
\text { Höhe der Bandscheibe Th } 3 / 4 \text {. } \\
\text { IHAs parallel zu } T_{X} \text {. } \\
\text { max. Lateralflexion: } \\
\text { Verkippen nach lateral zur Seite der } \\
\text { jeweiligen Neigungsrichtung. }\end{array}$ \\
\hline ventrale Vorlast & $\begin{array}{l}\text { IHAs v-förmig, geringe IHA-Wanderung. } \\
\text { IHAs parallel zu } T_{x} \text {. } \\
\text { max. Lateralflexion: } \\
\text { Verkippen nach lateral zur Seite der } \\
\text { jeweiligen Neigungsrichtung. }\end{array}$ & $\begin{array}{l}\text { IHAs verläuft in einem nach kranial } \\
\text { weit offenen Bogen auf der Höhe } \\
\text { der Bandscheibe Th } 3 / 4 \text {. } \\
\text { IHAs parallel zu } T_{x} \text {. } \\
\text { max. Lateralflexion: } \\
\text { Verkippen nach lateral zur Seite der } \\
\text { jeweiligen Neigungsrichtung. }\end{array}$ \\
\hline laterale Vorlast & $\begin{array}{l}\text { IHAs liegen kaudal auf der Seite der } \\
\text { jeweiligen Neigungsrichung. }\end{array}$ & $\begin{array}{l}\text { IHAs liegen kaudal auf der Seite der } \\
\text { jeweiligen Neigungsrichung. }\end{array}$ \\
\hline \multicolumn{3}{|c|}{ Verhalten von ROM und Steifigkeit } \\
\hline dorsale Vorlast & $\begin{array}{l}\text { ROM } \downarrow \\
\text { Hysterese } \downarrow \\
\text { minimale Steifigkeit } \uparrow\end{array}$ & $\begin{array}{l}\text { ROM } \uparrow \\
\text { Hysterese } \uparrow \\
\text { minimale Steifigkeit } \downarrow\end{array}$ \\
\hline ventrale Vorlast & $\begin{array}{l}\text { ROM } \downarrow \\
\text { Hysterese } \downarrow \\
\text { minimale Steifigkeit } \uparrow \text { geringfügig }\end{array}$ & $\begin{array}{l}\mathrm{ROM} \uparrow \\
\text { Hysterese } \uparrow \\
\text { minimale Steifigkeit } \downarrow \text { geringfügig }\end{array}$ \\
\hline laterale Vorlast & $\begin{array}{l}\mathrm{ROM} \uparrow \\
\text { Hysterese } \uparrow \\
\text { minimale Steifigkeit } \downarrow\end{array}$ & $\begin{array}{l}\mathrm{ROM} \uparrow \\
\text { Hysterese } \uparrow \\
\text { minimale Steifigkeit } \downarrow\end{array}$ \\
\hline
\end{tabular}

Tab.3.9: Zusammenfassung der Ergebnisse der Versuchsreihe „Lateralflexion“ für Th 2/3 A nach Resektion beider Artt. zygapophysiales. 


\section{Diskussion}

\subsection{Die Bewegungsstruktur eines Brustwirbelsegments Th 2/3}

Um die wesentlichen Erkenntnisse über die Bewegungsstruktur des Brustwirbelsegments Th 2/3 aufzeigen zu können, beginnt die Diskussion der Daten mit der Interpretation der Ergebnisse. Es werden die Ergebnisse ausgehend vom reduzierten Bewegungssegment nach Resektion der beiden Gelenke bei intakter Bandscheibe und weitgehend intakten Ligamenten bis hin zum intakten Brustwirbelsegment diskutiert. Dabei wird Bezug zur Anatomie und Physiologie hergestellt und die Mechanismen der Gelenkführung und ihre Bedeutung für die Funktion des Segments erörtert. Danach wird die Messmethode eingehend diskutiert und mit anderen Studien verglichen. Anschließend folgt ein kurzer Ausblick für weitere Messungen im Lichte der gewonnenen Informationen.

\subsubsection{Applikation eines axial ausgerichteten Drehmoments $T_{Z}(t)$ (Axiale Rotation)}

\subsubsection{Bandscheibe mit Bandapparat: Bewegungssegment nach Resektion der Wirbelbogengelenke}

Die momentanen Schraubachsen liegen größtenteils mittig und fast ortsfest in der Bandscheibe, wie es auch bei dem Bandscheibenmodell im Vorversuch der Fall war. Bei Bewegungsumkehr aber liegt die IHA außerhalb des Bandscheibenzentrums und wandert anschließend auf dieses zu. Diese exzentrische Wanderung ist durch eine visko-elastische Wirkung des lateralen Bandapparats und der Bandscheibe zu erklären, auch Einschwungvorgang genannt.

Einen veränderten Verlauf nehmen die Rastpolkurven bei einer Vorlastposition $\mathrm{XF}<-10 \mathrm{~mm}$ dorsal des Widerstandszentrums. Die Rastpolkurven für Hin- und 
Rücklauf eines Messzyklus enden zwar in der Bandscheibe, weisen aber dorsale Ausläufer auf, die mittig durch den Canalis vertebralis in die Bandscheibe ziehen. Aufgrund der anatomischen Besonderheiten des Brustwirbels wird die Bewegung des Bandscheiben-Bänder-Präparat durch den knöchernen Kontakt zweier benachbarter Dornfortsätzen limitiert. Es kann davon ausgegangen werden, dass durch dieses Impingement der Dornfortsätze zu einer Verlagerung der IHA nach dorsal kommt.

Eine Steifigkeitserhöhung für große Rotationswinkel wird nicht beobachtet. Die Schraubsteigung nimmt leicht $z u$. Die ansteigende Schraubsteigung lässt sich anlehnend an den anatomischen Aufbau der Bandscheibe folgendermaßen erklären: Bei ansteigendem Drehwinkel a kommt es zum Verdrillen des scherengitterförmigen Fasergeflecht des Anulus fibrosus, der den Bandscheibenkern (Nucleus pulposus) einschließt und diesen dadurch seitlich komprimiert (KAPANDJI 1985). Die Bandscheibe erfährt eine Höhenzunahme, wodurch sich die Endflächen zweier benachbarter Wirbelkörpern voneinander entfernen. Die Innenspannung im Nucleus pulposus führt zu einem rücktreibenden Drehmoment. Das „Anwachsen“ der Bandscheibendicke wird durch die axiale Torsion erzeugt, und zwar unabhängig von der Drehrichtung (NÄGERL ET AL. 1995).

Schlussfolgernd findet sich im Bereich physiologischer Drehwinkel ${ }^{1}$ $(\alpha= \pm 0,99$ ein ähnliches strukturelles Verhalten wie b ei einer Gummischeibe: Die momentane Schraubachse ist ortsfest.

\subsubsection{Bewegungssegment nach Resektion des rechten Wirbelbogen- gelenks}

Die Rastpolkurve bei zentraler Vorlastposition (0/0) folgt nun halbseitig der Rastpolkurve des intakten Segments (Abb. 3.2.12, S. 89). Bei einer Rechtsdrehung aus der Neutralstellung, Rotation in negativer Richtung von $0^{\circ}$ nach $-\alpha_{\text {Max }}$, verlagern sich die momentanen Schraubachsen auf die linke Seite. Bei einer Linksdrehung, Drehung mit positivem Vorzeichen aus der Neutralstellung von $0^{\circ}$ nach

\footnotetext{
${ }^{1}$ In der von GREGERSEN und LUCAS (1967) durchgeführten Messungen der Bewegungsamplituden zwei benachbarten Wirbelkörpern in vivo lagen die Durchschnittswerte bei der Axialrotation an den Th2/ Th3 zwischen 0,4 und 0,8 Grad für einen physiologischen Bewegungsbereich.
} 
$+\alpha_{\text {Max }}$, ist die Bahn, die die Schraubachsen durchlaufen, deutlich verkürzt. Die Wanderung beginnt am linken lateralen Bandscheibenrand und endet, wie bei dem Experiment „Bandscheibe mit Bandapparat“, mittig in der Bandscheibe, wo sie dann auch verbleibt.

Hierzu passen auch die anderen Schraubachsparameter: So zeigt die Schraubsteigung $\tau(\alpha)$ einen asymmetrischen Verlauf. Bei einer Linksrotation entspricht der Verlauf der Schraubsteigung ungefähr den Werten des BandscheibeBandapparats und bei einer Rechtsdrehung dem entsprechenden Betrag der Schraubsteigung des intakten Segments.

Diese auffällige Asymmetrie lässt sich folgendermaßen erklären: Aus der Neutralstellung $(\alpha=0)$ tritt bei der Drehung nach rechts das verbleibende linke Gelenk in Funktion. Es kommt zu einem Kraftschluss der krümmungsinkongruenten Gelenkflächen. Bei der Drehung aus der Neutralstellung nach links, wird im verbliebenen Gelenk der Kraftschluss gelöst und damit die Gelenkführung aufgehoben. Für bereits kleine Werte $\alpha=+1^{\circ}$ liegt eine nahezu ortsfeste Lokalisierung der Schraubachse in der Bandscheibe des Präparats vor. Die Wanderung der Schraubachse innerhalb der Bandscheibe wird durch die zunehmende Anspannung des lateralen Bandapparat bei zu großen Rotationswinkeln, die mit einer erhöhten Verkippung der IHA nach lateral und ventral einhergeht und durch einen knöchernen Kontakt von zwei benachbarten Dornfortsätzen noch verstärkt wird, verursacht.

Eine Steifigkeitserhöhung ist minimal.

\subsubsection{Intakte Bewegungssegmente}

Die Bewegungsstruktur des intakten Segments ist im physiologischen Bewegungsbereich weitgehend unabhängig von der Position der axialen Vorlast.

Die berechneten Schraubachsen sind im Drehwinkelbereich von $\alpha= \pm 1^{\circ}$ parallel zueinander und zum angreifenden Drehmoment $T_{z}$.

Das Verhalten aller Bewegungsparameter ist bei den beiden untersuchten Segmenten $\mathbf{A}$ und $\mathbf{B}$ annähernd gleich. Beide besitzen eine gebogene, nach ventral konvexe Form der Rastpolkurven, was unter der Annahme eines Viergelenks erwartet wird. Der qualitative Unterschied im Verlauf der Rastpolkurven beider Be- 
wegungssegmente ist gering und auf die Krümmungsmorphologie der Gelenkflächen sowie auf einen unterschiedlichen interartikulären Abstand zurückzuführen. Über verschiedene Formen des Viergelenks wurde bereits im Kapitel 1.4.3.3 (S. 39) berichtet.

Im physiologischen Drehwinkelbereich $\pm 1^{\circ}$ liegen die Rastpolkurven im gleichen Bandscheibenbereich und sind parallel zur z-Achse ausgerichtet. Diese Beobachtung gilt für alle gemessene Vorlastpositionen, auch für die extrem lateralen Vorlasten.

Bei ventralen Vorlastpositionen in den Rotationsmaxima kommt es zur einseitigen Führung der jeweiligen Bogengelenke, so dass bei einer Rechtsdrehung die linke Art. zygapophysialis und umgekehrt bei der Linksdrehung das rechte Bogengelenk kraftschlüssig wird und in Funktion tritt. Für die physiologischen Drehwinkel ( $\alpha= \pm 19$ befinden sich die Durchstoßpunkte der IHAs in der Bandscheibe. Die Gelenke entkoppeln und die Gelenkführung setzt aus. In dem Fall bestimmt das Widerstandszentrum der Bandscheibe die Position der momentanen Schraubachsen. Bei der Verschiebung der Vorlast nach dorsal verkippt das Segment in eine Extensionslage, somit treten beide Wirbelbogengelenke in Funktion. Die biomechanischen Eigenschaften im intakten Bewegungssegment bei zwei kraftschlüssigen Gelenken in einem festen Abstand zueinander lassen sich entsprechend dem einfachsten Modell zwei miteinander gekoppelter dimerer Gelenkketten erklären. Die Wirbelbogengelenke sind zu einem symmetrischen Viergelenk gekoppelt (NÄGERL 1990, Kubein-MeESEnBURG et AL. 1991a, NÄGeRL ET AL. 1992).

Im physiologischen Drehwinkelbereich $(\alpha= \pm 19$ und bei einer extensiven Vorlast sind beide Gelenke kraftschlüssig. Steigt der Drehwinkel $\alpha$, so verändern sich die Bedingungen des Kraftschlusses in beiden Gelenken. Bei der Linksdrehung für Winkel $\alpha>+1^{\circ}$ wird das rechte Bogengelenk zunehmend belastet und das linke Gelenk mit ansteigender Drehung entlastet, so dass schließlich nur noch das rechte Gelenk führt. Das entlastete Bogengelenk entkoppelt aber nicht vollständig, so dass eine ständige Führung beider Gelenke gegeben ist. Bei der Richtungsumkehr springt die Rastpolkurve nach dorsal und wandert nun bei negativen Drehwinkeln wieder nach ventral in die Bandscheibe bis $\alpha=-1^{\circ}$. 
Schlussfolgernd wird die Bewegungsstruktur bei dorsalen Vorlasten im kompletten Drehwinkelbereich durch beide Bogengelenke bestimmt. Die Steifigkeit ist erhöht.

Bei zentralen Vorlastpositionen liegen die Durchstoßpunkte der momentanen Schraubachsen für den Drehwinkel $\alpha= \pm 1^{\circ}$ mittig in der Bandscheibe. Der Wanderungsbereich der IHA ist sehr klein und beträgt sowohl in $\mathrm{x}$ - als auch in y-Richtung nur $4 \mathrm{~mm}$. Diese geringe Wanderung spricht für das Ausbleiben einer Gelenkführung im physiologischen Bewegungsbereich. Somit sind die Wirbelbogengelenke in der Neutralstellung entkoppelt. In maximaler Rechtsdrehung führt das linke Gelenk und das rechte Gelenk entkoppelt und umgekehrt. Die Gelenke wechseln sich in der Führung ab.

Um das Verhalten der IHA bei einer Verschiebung der Kraftwirkungslinie in der Sagittalebene zu verdeutlichen, werden hier ihre Position $(x / y)$ bei $\alpha=0^{\circ}$ und die Wanderungsstrecke für den physiologischen Drehwinkelbereich ( $\alpha= \pm 19$ tabellarisch zusammengefasst.

\begin{tabular}{|c|c|c|c|}
\hline \multirow{2}{*}{ Position der Vorlast } & \multicolumn{2}{|c|}{ Lage der IHA bei $\alpha=0^{\circ}$} & Wanderungsstrecke \\
\cline { 2 - 4 } & X-Koordinate & Y-Koordinate & der IHA bei $\alpha= \pm 1^{\circ}$ \\
\hline zentral & $10 \mathrm{~mm}$ & $0 \mathrm{~mm}$ & $1-2 \mathrm{~mm}$ \\
\hline dorsal & $9 \mathrm{~mm}$ & $0 \mathrm{~mm}$ & $2 \mathrm{~mm}$ \\
\hline ventral & $10 \mathrm{~mm}$ & $0 \mathrm{~mm}$ & $2-3 \mathrm{~mm}$ \\
\hline
\end{tabular}

Die Koordinaten der Durchstoßpunkte der Schraubachsen mit der xy-Ebene für $\alpha=0^{\circ}$ und bei allen gemessenen Vorlastpositionen sind nahezu ortsfest, und liegen mittig in der Zwischenwirbelscheibe, etwa $10 \mathrm{~mm}$ ventral des ermittelten Widerstandszentrums. Das gilt für beide untersuchten Bewegungssegmente.

\subsubsection{Funktionelle Zusammenhänge}

Im Experiment „Axialrotation“ deutet der Rastpolkurvenverlauf auf ein Viergelenk hin. Sind die Gelenke kraftschlüssig, so ist eine Zwangsführung im Sinne einer Viergelenkkette gegeben. Durch die spezielle Anordnung der Wirbelbogengelenke mit ihren leicht nach ventral gekrümmten Gelenkflächen liegen die entsprechen- 
den Radienmittelpunkte im Bereich der Zwischenwirbelscheibe. Es wird eine einfache dimere Viergelenkkette gebildet (Kapitel 1.4.3.3, S. 37).

In Anlehnung an den anatomischem Aufbau des Junghannsschen Segments Th2/3 und mit Hilfe der Gelenkmechanik lässt sich die Bewegungsstruktur nun wie folgt erklären: die Gelenkführung in den Brustwirbelsegmenten ist so aufgebaut, dass unabhängig von der Position der axialen Vorlast bei einer Drehung im physiologischen Bewegungsbereich die Durchstoßpunkte der momentanen Schraubachsen stets in der Bandscheibe liegen.

Betrachtet man die Lage und die Wanderung der Rastpolkurven in einem physiologischen Bewegungsbereich ( $\alpha= \pm 19$ bei intaktem Segment und nach sukzessiver Resektion von Gelenken, so kann man eine Aussage über die Rolle der Wirbelbogengelenke bei der Bewegungsführung treffen: Der Einfluss der Gelenkführung auf die Lage der momentanen Schraubachse scheint marginal zu sein.

Die Erklärung kann nun folgend sein: Für physiologische Bewegungsintervale ist die komplexe Krümmungsmorphologie der Gelenkflächen so gestaltet, dass bei Einpunkt-, Mehrpunktkontakt oder bei einer Entkoppelung der Gelenke die Rastpolkurve stets im Zentrum der Bandscheibe zu liegen kommt, wie es bereits auch von LEE für Th6/7-Segment beobachtet wurde.

\begin{tabular}{|c|c|c|c|}
\hline \multirow{2}{*}{} & \multicolumn{2}{|c|}{ Lage der IHA bei $\alpha=0^{\circ}$} & Wanderungsstrecke \\
\cline { 2 - 3 } & X-Koordinate & Y- Koordinate & der IHA bei $\alpha= \pm 1^{\circ}$ \\
\hline Intaktes Segment & $10 \mathrm{~mm}$ & $0 \mathrm{~mm}$ & $1-2 \mathrm{~mm}$ \\
\hline Res. re. Gelenk & $10 \mathrm{~mm}$ & $0 \mathrm{~mm}$ & $1-2 \mathrm{~mm}$ \\
\hline Res. re./li. Gelenk & $10 \mathrm{~mm}$ & $0 \mathrm{~mm}$ & $1-2 \mathrm{~mm}$ \\
\hline
\end{tabular}

Die Koordinaten der Durchstoßpunkte der Schraubachsen mit der xy-Ebene bei $\alpha=0^{\circ}$ und bei allen gemessenen Vorlastpositionen sind ortsfest und liegen mittig in der Bandscheibe, etwa $10 \mathrm{~mm}$ ventral des ermittelten Widerstandszentrums.

Die Lage der momentanen Schraubachse bleibt nach Resektion des rechten und dann des linken Bogengelenks nahezu unverändert. Schlussfolgernd haben die Gelenke kaum Einfluss auf die Lage der momentanen Schraubachse.

Damit korrelierend verändert sich nach sukzessiver Resektion der Artt. zygapophysiales die differenzielle Steifigkeit in physiologischen Bewegungsbereich 
$(\alpha= \pm 19$ kaum (Abb. 3.2.19 Seite 93). Anders als im Le ndenbereich (MANSOUR 2001) wo eine massive Steifigkeitserhöhung durch die Gelenkführung bedingt ist und den Bewegungsumfang eingrenzt, hat die Natur in der Brustwirbelsäule auf diesen Versteifungsmechanismus verzichtet.

Schlussfolgernd kann man sagen, dass die Gelenkführung die momentane Lage der IHA nicht oder kaum beeinflussen kann. Auf eine Erhöhung der Segmentsteifigkeit bei einer unphysiologischen Belastung zum Schutz der Bandscheibe und der Spinalnervenwurzeln wird verzichtet, da eine ausreichende Stabilisierung des Segments durch die Brustkorbaufhängung gegeben ist.

\subsubsection{Applikation eines Drehmoments parallel zur y-Achse (Flexion/ Extension)}

\subsubsection{Bandscheibe mit Bandapparat: Bewegungssegment nach Resektion der Wirbelbogengelenke}

Die gemessenen Werte der Versuchsreihe Flexion/Extension zeigen eine Wanderung der momentanen Schraubachsen von ventral nach dorsal mitten in dem Wirbelkörper des Th 3 und auf der Höhe der Artt. zygapophysiales des Bewegungssegmentes Th 3/4. Die Rastpolkurven für Extension und Flexion fallen praktisch zusammen. Die Rastpolkurve ist für die Drehwinkel $\alpha= \pm 1^{\circ}$ nahezu ortsfest. In maximaler Extensionsstellung verkippen die momentanen Schraubachsen leicht nach ventral.

Zusammenfassend kann man sagen, dass es bei Extension zu einer Stauchung des Ligamentums interspinale kommen kann, so dass sich die Dornfortsätze berühren und als unphysiologisches Widerlager in der Extensionsbewegung in Erscheinung treten. 


\subsubsection{Bewegungssegment nach Resektion des rechten Wirbelbogen- gelenks}

Der Verlauf der Rastpolkurve ist v-förmig. Die Durchstoßpunkte der momentanen Schraubachsen liegen auf einer Bahn, die knapp oberhalb der Zwischenwirbelscheibe des Th3/4-Segments verläuft. Die Rastpolkurve wandert für die Flexionslage $(\alpha>0)$ schräg nach kranial in Richtung der Bandscheibe, während sie bei Extensionslage $(\alpha<0)$ leicht nach oben in die Richtung des verbliebenen linken Gelenks zieht. Der Bewegungsumfang in Extension ist reduziert $\left(\alpha=-1,2^{\circ}\right)$ und die Steifigkeit bleibt nahezu unverändert. In Flexionsrichtung ist sowohl der Bewegungsumfang als auch die Steifigkeit nur geringfügig erhöht. Nach Resektion des rechten Art. zygapophysiales kommt es zu einer leichten Verkippung der Schraubachsen bei der Extensionsbewegung, so dass der obere Wirbelkörper nach rechts unten $\left(e_{z}>0\right)$ und nach dorsal schwenkt. Diese Schwenkung ist mit geringfügiger axialer Rotation verbunden, was wiederum die Anspannung des Bandapparates mit sich bringt und zur exzentrischen IHA- Wanderung führt.

\subsubsection{Intakte Bewegungssegmente}

Bei beiden gemessenen Bewegungssegmenten verläuft die Rastpolkurve knapp oberhalb der Bandscheibe des vierten thorakalen Wirbels nach dorsal in Richtung der Wirbelbogengelenke. Die Wanderung der Schraubachsen in sagittaler und in vertikaler Richtung ist gering ( $\Delta \mathrm{X}=4 \mathrm{~mm}, \Delta \mathrm{z}=4 \mathrm{~mm})$, so dass für die physiologischen Drehwinkel $\alpha= \pm 1^{\circ}$ die gemessenen momentanen Schraubachsen nahezu ortsfest oberhalb der Zwischenwirbelscheibe des Th3/4 liegen. Eine Verschiebung der axialen Vorlasten sowohl nach ventral/ dorsal als auch nach lateral verändert die Bewegungsparameter kaum.

Die Drehwinkel-Drehmoment-Kennlinie besitzt für zentrale Vorlasten annähernd sigmoide Form mit einem Bewegungsumfang von $4^{\circ}$. Bei den statischen Messungen (Kap. 3.3.1.2, S. 120) weicht die Drehwinkel-Drehmoment-Kennlinie bei extremen dorsalen/ventralen Vorlastpositionen von ihrer sigmoiden Form ab, so dass sie eher ein hockeyschlägerförmiges Aussehen besitzt. Im zweiten Segment Th 2/3 B zeigte sich bei ventralen Vorlasten eine fast lineare $\alpha(T)$-Funktion (Kap. 3.3.2.2). Die gewisse Annäherung an die elastische Grenze, die zur Steifigkeits- 
erhöhung führen soll, ist nicht vorhanden oder minimal, so dass die Hysteresekurven keine Sigmoidität aufwiesen. Die Parameter deuten auf einen rein viskoelastischen Effekt.

Die Richtungsvektoren sind nahezu parallel zu dem angreifenden Drehmoment ausgerichtet. Die Schraubsteigung beträgt $0,24 \mathrm{~mm} /{ }^{\circ}$.

Bei der Flexion bestimmt das Widerstandszentrum der Bandscheibe die Bewegungsstruktur. Die Wirbelbogengelenke sind entkoppelt.

Bei der Extension sind die Wirbelbogengelenke kraftschlüssig, die aufgrund ihrer dachziegelartigen Anordnung schon sehr bald in Kontakt miteinander treten (KAPANDJI 1985). Der Extensionswinkel ist gering und beträgt für zentrale Vorlast nur 1․ Die minimale Steifigkeit steigt in der Exte nsion im Vergleich zur Flexion auf mehr als 60\% (Abb. 3.3.9, S.123). Die Kraftschlüssigkeit der Gelenke bestimmt die Bewegungsstruktur und die Steifigkeit im Bewegungssegment.

\subsubsection{Funktionelle Zusammenhänge}

Die beiden Wirbelbogengelenke lassen sich durch zwei dimere Kugelgelenkketten in parallelen Ebenen beschreiben. In Extensionslage kommt es zum Kraftschluss der Gelenkflächen. Daraus resultiert eine Erhöhung der Steifigkeit. Bedingt durch die Anatomie der Brustwirbelsäule, insbesondere die beginnende Kyphose im Bereich Th2/3, ist die Extensionsbewegung sehr gering. Sie beträgt für zentrale Vorlast nur $|\alpha|=1$. Bei dorsalen Vorlasten ist sie noch geringe $r|\alpha|=0,45^{\circ}$ (Abb. 3.3.7, S. 122). Bei Flexion entkoppeln die Wirbelbogengelenke und ermöglichen eine hohe Flexibilität des Bewegungssegments.

Bei beiden gemessenen Bewegungssegmenten liegen die momentanen Schraubachsen nicht in der Bandscheibe des untersuchten Bewegungssegments, wie es bei LEE (2003) der Fall war, sondern verlaufen auf einer Bahn, die knapp oberhalb der Zwischenwirbelscheibe des Th3/4 Segments zu liegen kommt. Dieser Verlauf ist wie gefolgt zu erklären: Aufgrund der dachziegelartigen Anordnung der Gelenkflächen liegen die entsprechenden Radienmittelpunkte bei zwei intakten Wirbelbogengelenken in der Flexion ungefähr im Gebiet der Bandscheibe des unmittelbar darunter liegenden Bewegungssegments. Es kann angenommen werden, dass die 
zwei benachbarten Bewegungssegmente Th2/3 und Th3/4 eine gemeinsame Flexionsachse besitzen und somit eine funktionelle Einheit bilden.

Betrachtet man die Lage der Rastpolkurve bei $\alpha=0^{\circ}$ und ihre Wanderungsstrecke bei Flexions-, Extensionsbewegung in einem physiologischen Drehwinkelbereich $\alpha= \pm 1^{\circ}$ bei intaktem Segment und nach sukzessiver Rese ktion von Gelenken, so kann man einen „Effekt der Wirbelbogengelenke“ beobachten.

\begin{tabular}{|c|c|c|c|}
\hline \multirow{2}{*}{} & \multicolumn{2}{|c|}{ Lage der IHA bei $\alpha=0^{\circ}$} & Wanderungsstrecke \\
\cline { 2 - 4 } & X-Koordinate & Z- Koordinate & der IHA bei $\alpha= \pm 1^{\circ}$ \\
\hline Intaktes Segment & $6 \mathrm{~mm}$ & $-22 \mathrm{~mm}$ & $2 \mathrm{~mm}$ \\
\hline Res. re. Gelenk & $4 \mathrm{~mm}$ & $-18 \mathrm{~mm}$ & $1-2 \mathrm{~mm}$ \\
\hline Res. re./li. Gelenk & $4 \mathrm{~mm}$ & $-14 \mathrm{~mm}$ & $1-2 \mathrm{~mm}$ \\
\hline
\end{tabular}

Bei einer sukzessiven Resektion der Artt. zygapophysiales kommt es zur einen sukzessiven Hochwanderung der Rastpolkurve nach cranial, wobei ihr Wanderungsbereich reduziert erscheint.

\subsubsection{Applikation eines Drehmoments parallel zur x-Achse (Lateralflexion)}

\subsubsection{Bandscheibe, mit Bandapparat: Bewegungssegment nach Resektion der Wirbelbogengelenke}

Die momentanen Schraubachsen sind nicht ortsfest. Die Bahn der Rastpolkurve verläuft kaudal der Bandscheibe des Th2/3- Segments. Bei einem absoluten Drehwinkel $\alpha= \pm 0,9^{\circ}$ liegen die Durchstoßpunkte der IHA mittig und knapp unterhalb der Bandscheibe Th3/4. Innerhalb eines Messzyklus (Neigung nach rechts/ links) wandert die Rastpolkurve über einen Bereich von $\pm 8 \mathrm{~mm}$ in lateraler und $\pm 4 \mathrm{~mm}$ in vertikaler Richtung (Abb.3.4.7 B, S. 143). Bei Rotationswinkeln $|\alpha|>1,8^{\circ}$ findet eine geringfügige Wanderung nach kranial statt.

Die Wanderung der IHA ist folgendermaßen zu erklären: Bei positiven Drehwinkel wird der Bandapparat auf der linken Seite gespannt und auf der rechten Seite ent- 
spannt und das Widerstandszentrum bewegt sich nach links, so dass die IHA auf die rechte Seite wandert. Für negative Drehwinkel ist das Verhalten umgekehrt.

Die $e_{Y}(\alpha)$-Komponente des Richtungsvektors steigt je nach Neigungsrichtung an und die momentane Schraubachse verkippt in die Richtung der Seitwärtsbewegung. Für den kompletten Rotationsbereich $\left(|\alpha|=3,9^{\circ}\right)$ wird eine symmetrische $\alpha(T)$-Abhängigkeit beobachtet. Die Links- oder Rechtslage resultiert nur in einem geringfügigen Anstieg der Steifigkeit. Eine Verschiebung der Vorlast bewirkt keine nennenswerte Änderung der Kinematik des Bandscheibe-BandapparatPräparates.

Bei der Seitwärtsneigung verkippt der obere Wirbelkörper und die Bandscheibe wird sowohl auf Dehnung/ Stauchung als auch auf Scherung beansprucht.

\subsubsection{Bewegungssegment nach Resektion des rechten Wirbelbogen- gelenks}

Die momentanen Schraubachsen für beide Neigungsrichtungen liegen auf der Höhe der Bandscheibe des Bewegungssegments Th 3/4 (Abb. 3.4.7 A, S. 143). Bei einem absoluten Drehwinkel $\alpha= \pm 0,9^{\circ}$ liegen die Durchstoßpunkte der momentanen Schraubachsen mittig in der Zwischenwirbelscheibe Th 3/4. Die Lage der Rastpolkurve und ihr Verlauf sind mit denen des Bandscheibe-BandapparatPräparats nahezu identisch. Es können keine wesentlichen Änderungen der Bewegungsparameter bei einer Links- oder Rechtsneigung registriert werden.

\subsubsection{Intakte Bewegungssegmente}

Die Rastpolkurven $r(\alpha)$ von den beiden gemessenen Bewegungssegmente Th 2/3 A und B zeigen keine deutliche Unterschiede (Kapitel 3.4.1, S. 139). Die Rastpolkurven des zweiten Bewegungssegments Th 2/3 B verlaufen etwa $9 \mathrm{~mm}$ weiter kranial als die des Th 2/3 A-Segments. Sie sind deutlich komprimierter und ihr Wanderbereich ist kleiner, was auch den anatomischen Gegebenheiten des Th 2/3 B Bewegungssegmentes entspricht. Die Bewegung ist eben und gleichmäßig. Eine Änderung der Bewegungsstruktur für ventrale/ dorsale Vorlastpositionen im Vergleich zu den zentralen Vorlastpositionen wird nicht beobachtet. 
Die Rastpolkurve wandert für eine Lateralflexion nach links $(\alpha<0)$ zur linken Seite und für eine Flexion nach rechts $(\alpha>0)$ aus der Mitte zur rechten Seite.

Mit der Lateralflexion findet begleitend eine geringere axiale Rotation statt. So korreliert eine Lateralflexion nach links mit einer Rechtsrotation des oberen Wirbels und umgekehrt.

Das applizierte Drehmoment $T_{X}$ führt zu einer beinahe reinen Seitwärtsneigung des Segments. Dennoch ist eine enorme Wanderung der IHA (ca. $\pm 12 \mathrm{~mm}$ ) zu verzeichnen, so dass neben der Bandscheibe und dem lateralen Bandapparat eine Beeinflussung durch die Wirbelbogengelenke angenommen werden muss.

Zusammenfassend kann man sagen, dass die Gelenke unabhängig von der Vorlastposition (auch bei extremen lateralen Vorlasten) die Bewegungsstruktur des Segments bei der Lateralflexion mit beeinflussen.

\subsubsection{Funktionelle Zusammenhänge}

Bei einem Drehwinkelbereich $\alpha= \pm 0,9^{\circ}$ liegen die Durchstoßpunkte der IHA mittig und knapp oberhalb der Bandscheibe Th3/4-Segmens. Die Wanderung der IHA für ein geringen Winkelbereich $(|\alpha|=1,89$ ist aber enorm, so dass eine Beteiligung der Wirbelbogengelenke bei der Seitwärtsbewegung als sicher erscheint. Im Allgemeinen wird die Rolle der Wirbelbogengelenke bei Lateralflexion Aufgrund ihrer frontal gestellten Gelenkfacetten eher als unbedeutend angesehen.

Hier soll jetzt genauer auf die mögliche Bedeutung der Gelenke eingegangen werden: Die Lage der Rastpolkurve bei $\alpha=0^{\circ}$ im Koordinatensystem (y/z) und ihre Wanderungsstrecke bei der Seitwärtsbewegung in einem physiologischen Drehwinkelbereich $\alpha= \pm 0,9^{\circ}$ bei einem intakten Bewegungssegment und nach sukzessiver Resektion von Gelenken wird nochmals tabellarisch zusammengefasst.

\begin{tabular}{|c|c|c|c|}
\hline \multirow{2}{*}{} & \multicolumn{2}{|c|}{ Lage der IHA bei $\alpha=0^{\circ}$} & Wanderungsstrecke \\
\cline { 2 - 3 } & Y- Koordinate & Z- Koordinate & der IHA bei $\alpha= \pm 1^{\circ}$ \\
\hline Intaktes Segment & $0 \mathrm{~mm}$ & $-23 \mathrm{~mm}$ & $12 \mathrm{~mm}$ \\
\hline Res. re. Gelenk & $0 \mathrm{~mm}$ & $-18 \mathrm{~mm}$ & $9 \mathrm{~mm}$ \\
\hline Res. re./li. Gelenk & $0 \mathrm{~mm}$ & $-22 \mathrm{~mm}$ & $7 \mathrm{~mm}$ \\
\hline
\end{tabular}


Analog der Flexions-, Extensionsbewegung führt die sukzessive Resektion der Artt. zygapophysiales zum Einen zu einer Verlagerung der Rastpolkurve nach kranial. Zum Anderen spricht die Reduktion der Wanderungstendenz von $12 \mathrm{~mm}$ auf $7 \mathrm{~mm}$ für eine tatsächlich vorhandene Beteiligung der Wirbelbogengelenke an der Bewegungsführung. Des Weiteren wird vermutet, dass der Ausfall eines Bogengelenks zu einer Verschiebung der Flexionsachse nach kranial führt und beim Verlust von beiden Wirbelbogengelenken das unmittelbar darunter liegender Bewegungssegment bei der Führung der Seitwärtsbewegung mitbeteiligt wird.

Analog der Flexion/ Extension wird auch bei der Lateralflexion eine gemeinsame Flexionsachse vermutet, welche die zwei benachbarten Bewegungssegmente Th2/3 und Th3/4 zu einer funktionellen Einheit verbindet.

\subsection{Methodendiskussion}

\subsubsection{Kraftsystem}

Das Ausmaß der physiologischen Belastung der Wirbelsäule durch die auf das Bewegungssegment einwirkenden Muskelkräfte und durch die Gewichtkraft des Kopfes ist schwer abzuschätzen. Die Komplexität dieses Kraftsystems kann auf eine Einzelkraft (axiale Vorlast $F_{Z}$ ) und ein Kräftepaar mit einem zu $F_{Z}$ parallelen Drehmomentvektor (Drehmoment $T_{z}$ ) reduziert werden. Dieses Kraftsystem wird dann als eine Kraftschraube bezeichnet (Kapitel 2.4). Somit werden in dieser Studie alle angreifenden Kräfte auf verschiedene Kraftschrauben reduziert. Die Kraftschraube mit ihren 6 Parametern (drei Kräfte und drei Drehmomente) lässt sich leicht kontrollieren, so dass unabhängig von einer Vielzahl an Kräftekonstellationen ihre Simulation und Beeinflussung stets reproduzierbar und konstant ist. Unter in dieser Arbeit gewählten Versuchsanordnung bleibt eine Führung der Bewegung durch die Kraftschraube aus.

Eine Systematisierung von Muskelkräften wurde schon im Kapitel 1.2 „Anatomie“ (S. 8) vorgenommen, wobei die Rücken- und Thoraxmuskulatur in funktionellen 
Gruppen mit ähnlicher Wirkung eingeteilt wurden. Die Aufbringung einer Einzelkraft, die ein Muskel oder eine Muskelgruppe repräsentiert, würde nicht nur den Versuchsrahmen sprengen, sondern auch die dem Versuch relevanten Parameter soweit beeinflussen, dass eine Datenerhebung und ihr folgende Analyse praktisch unmöglich wären. Eine geringe Änderung der Kraftwirkungslinie würde zu einer Änderung der Druckbelastung in den Gelenken führen (EL-BOHY ET. AL. 1989) und somit zu einer veränderten Gelenkführung mit einem anderen Verhalten des Systems.

Eine Abschätzung der Wirkung einer Muskelkraft und ihre direkte Quantifizierung mit Hilfe eines EMGs sind aufgrund äußerer Störungen schwierig (RALSTON 1961). Zudem ist die Muskelkraft in vivo nicht konstant und sie kann kaum mit mehr als mit 15\% ihrer Maximalkraft dauerhaft wirken (PARNIANPOUR ET AL. 1989).

Also ist es physikalisch gesehen nicht nur zulässig, sondern im Sinne einer gezielten Beeinflussung von einzelnen Parametern auch zwingend notwendig die Muskelkräfte und die Gewichtkraft des Kopfes und des Oberkörpers auf eine Kraftschraube zu reduzieren.

\subsubsection{Die kompressive Kraftkomponente}

Die Bedeutung einer axialen Vorlast als simulierte Muskelkraft wird durch zahlreiche theoretische Überlegungen und Untersuchungen bestätigt (PANJABI ET AL. 1974, LiN ET AL. 1978, MilleR UND Skogland 1980, Goel ET AL. 1987, Aвumi ET AL. 1990, AhMEd ET AL. 1990, GoodWIN ET AL. 1994, SCHILDHAUER ET AL. 1994, PATWARDHAN ET AL. 2002, WILKE ET AL. 2002).

In dieser Arbeit wird eine frei hängende axiale Vorlast von 200N gewählt. Diese kompressive Kraft $F_{Z}$ sollte die Muskelkraft und die Gewichtkraft des Kopfes simulieren. Es wird gezeigt, dass eine weitere Erhöhung der axialen Vorlast zu keinen nennenswerten Unterschieden in der Bewegungsstruktur der Brustwirbelsegmente führt (Kap. 3.2.1.2, S. 82). Folgend wird in diese Studie auf die höhere Vorlasten verzichtet, um eine Schädigung des Bewegungssegments zu vermeiden. 


\subsubsection{Drehmoment}

In mehreren oben erwähnten Studien werden Drehmomente verschiedener Größenordnung aufgebracht. So wird beispielsweise bei PANJABI ET AL. (1976) mit maximal $8000 \mathrm{Ncm}$ gemessen. Auch bei WILKE (1993) werden sehr hohe Drehmomente aufgebracht, wobei das Präparat nach Erreichen einer „plastischen Zone" von der Belastungs-Verformungskurve irreversibel verformt wird. Sollen allerdings die Ergebnisse reproduzierbar bleiben, muss eine bleibende Schädigung des Bewegungssegments vermieden werden. Möchte man die vergleichenden Messungen mit demselben Präparat durchführen, dann muss das maximale Drehmoment so ausgelegt sein, dass der maximale physiologische Bewegungsumfang erreicht wird.

Bei beiden in dieser Studie untersuchten Brustwirbelsegmenten entspricht die sigmoide Form der Drehwinkel-Drehmoment-Kennlinien dem Verlauf der LastDeformationskurven von WILKE (1993), PANJABI ET AL. (1981) und MYERS ET AL. (1991) und deutet auf das Ende der physiologischen Bewegung hin.

In Kapitel „Material und Methode“ wird ausführlich gezeigt, dass mit Hilfe der verwendeten Messapparatur die Applikation eines reinen Drehmoments genau umgesetzt werden kann, wobei eine Führung der Bewegung des Messobjekts durch die Drehmomentwaage ausgeschlossen wird.

\subsection{Messsystem}

Um die komplexe Kinematik von den Wirbelbogengelenken zuverlässig und wirkungsvoll beschreiben zu können, ist es notwendig die Bewegungsstruktur hochaufgelöst und präzise mittels differentiell kleiner Winkel zu erfassen. Diese Forderung wird in dieser Studie mit Hilfe der beschriebenen Messtechnik erfüllt.

Die Erfassung von solch kleinen differentiellen Bewegungen ist nicht einfach, da sich schon die kleinsten Fehler in der Durchführung in der Datenanalyse fortpflanzen und die Ergebnisse verfälschen. 
Zur Abschätzung der Leistungsfähigkeit der in dieser Arbeit verwendeten Messapparatur wird zuerst die Positionierung der Lagesensoren im Halterahmen betrachtet. Prinzipiell wird die Anbringung der Lagesensoren als das größte Problem in der Messtechnik behandelt (SCHÄFER 1995). Die nicht exakte Positionierung der Messtaster im raumfesten Koordinatensystem (Halterahmen) kann einen möglichen systematischen Fehler mit sich bringen, was unter anderem an den Fertigungstoleranzen der Apparatur liegen kann. Die am Messobjekt eingelesenen Tasterwerte sind valide und die eingelesenen Daten sind numerisch am wenigsten sensibel. Hingegen ist die Berechnung der Rastpolkurven und der Schraubsteigung mit einer hohen Fehlerfortpflanzung behaftet.

\subsection{Wahl der Präparate}

Für biomechanische Untersuchungen müssten die Präparate idealerweise von jungen Erwachsenen stammen, die atraumatisch starben und bei denen man alle strukturelle Veränderungen ausschließen kann. Die Verfügbarkeit dieser Präparate ist gerade im Wirbelsäulenbereich kaum gegeben. Damit musste nach Alternativen gesucht werden, um der Schwierigkeit der Verfügbarkeit frischer humaner Wirbelsäulenpräparate auszuweichen. Die verfügbaren Präparaten stammen überwiegen von älteren Patienten. Ein Ausschluss von strukturellen Schäden erfolgte durch die Röntgen- und CT-Aufnahmen. Nach jedem Resektionsschnitt wurden neue Röntgenaufnahmen angefertigt und das Präparat auf Unversehrtheit nochmals untersucht.

Es wird nach Ansätzen gesucht, die fixierten humanen Präparate haltbar zu konservieren. Bei der chemischen Fixation beschreiben WILKE ET AL. (1996) eine Abhängigkeit der Segmentsteifigkeit von der Zusammensetzung der Fixationslösung. So eine Konservierung sollte die Präparate für eine kinematische Untersuchung unbrauchbar machen. Die Aussage kann so nicht stimmen, da eine Änderung der Segmentsteifigkeit keinen Einfluss auf die Bewegungsstruktur bedeutet. Die Bewegungsstruktur ist allein durch die Gelenkflächenmorphologie vorgegeben 
(NÄGERL ET AL. 1992, NÄGERL ET AL. 1995, KARLSCHEUER 2001, LEE 2003, WACHOWSKI ET AL. 2009 a, b).

Die Experimente an frischen Halswirbelsäulenpräparaten zeigen eine geringere Steifigkeit in Vergleich zu konservierten Präparaten (BOCKERMANN 2001, WACHOWSKI 2005). Die niedrige Steifigkeit gibt das maximal angreifende Drehmoment vor. So ist ein kleines Drehmoment ausreichend, um den physiologischen Bewegungsbereich des Segments zu überstreichen. Qualitativ ist die Abhängigkeit allerdings gleich (MANSOUR 2001). Unter der Bedingung, dass die Konservierungslösung die knöchernen Strukturen nicht angreift, bleibt die Führungsdominanz der Gelenke erhalten. In dieser Arbeit wurde bänderschonende Fixationslösung verwendet (FANGHÄNEL UND SCHULZ 1962), so kann man davon ausgehen, dass die Bewegungsstruktur beider Brustwirbelpräparate unverändert wiedegegeben wird. Pathologische Veränderungen an den beiden Präparaten durch Lagerung oder experimentelle Beanspruchung während der Versuchsdurchführung wurden nicht beobachtet.

\subsection{Ausblick}

Die in dieser Arbeit vorgestellte Messapparatur ist bis jetzt einzigartig. Mit ihr ist es möglich, eine hohe räumliche und zeitliche Auflösung der Bewegungsstruktur der Wirbelsäule zu erfassen und anschließend zu analysieren. Hier wird die Bewegung von zwei Segmenten der oberen Brustwirbelsäule analysiert, die noch eine vergleichsweise höhere Beweglichkeit aufweisen als die des mittleren Brustwirbelsäulenabschnitts (LEE 2003).

Es sollen demnächst die Brustwirbelsäulensegmente segmental ausgehend von Th3/4 bis zu den von LEE (2003) untersuchten Th6/7-Segmenten nacheinander untersucht werden, um in dieser Arbeit aufgestellte Hypothese zur Bildung einer Funktionellen Einheit zwischen zwei benachbarten Bewegungssegmenten zu bestätigen (oder auch zu widerlegen). Ein weiterer Gedankenschritt wäre es, die durch die zukünftigen biomechanischen Untersuchungen (Th3/4 bis Th 5/6) ge- 
wonnene Ergebnisse additiv zu einer Bewegung der „Durchschnittsbrustwirbelsäule" zusammenzufassen und zu prüfen, ob die funktionellen Verknüpfungen in der und zu der gesamten Brustwirbelsäule gegeben sind.

Generell wäre es wünschenswert, die gewonnenen Daten mit einer Versuchsreihe am frischen Wirbelsäulenmaterial zu vergleichen. Es werden sich zwar keine prinzipiellen Änderungen in der Bewegungsstruktur zeigen, jedoch könnte man die absoluten physiologischen Parameter von elastischen Segmentanteilen wie die der Zwischenwirbelscheibe präziser quantifizieren. Somit wäre es möglich, die kompletten biomechanischen Eigenschaften des Segmentes quantitativ zu durchleuchten.

Eine Messung von mehrsegmentalen Wirbelsäulenabschnitten könnte man nach entsprechendem Umbau der Apparatur weitgehend realisieren. Dadurch wäre es möglich, die Wechselwirkung von benachbarten Bewegungssegmenten nochmals zu durchleuchten und unter anderem einen Einfluss von pathologischen Veränderungen wie Skoliose zu analysieren. In dieser Hinsicht kann die Apparatur zu den heutigen Zeiten der Implantatforschung wichtige Erkenntnisse über die biomechanische Auswirkung der Bandscheibenprothesen sowie anderen OP-Verfahren, z.B. eine Versteifung von zwei und mehreren Bewegungssegmenten, auf die Änderung der Kinematik des darunterliegenden und noch beweglichen Segmente liefern.

Sollten die Messungen am komplexen System erfolgen, so könnte unter anderem ein altersabhängiger Vergleich der Kinematik der Wirbelsäule im Zusammenhang mit einer morphologischen Untersuchung einen Aufschluss über Veränderungen im Laufe des menschlichen Lebens geben.

In Bezug auf die Messapparatur sollte die Zahl der Lagesensoren erhöht werden, um die Messgenauigkeit noch weiter zu steigern. Die derzeitige Tasterkonfiguration ist für die die Axialrotation optimiert. Durch die Integration von zusätzlichen Tastern könnte man die drei Raumebenen des Koordinatensystems unabhängig voneinander bestimmen und so das Fortpflanzen von systematischen Fehlern minimieren. Die Messapparatur selbst wurde während der Studie modernisiert. Das Drehkreuz und die Halterungselemente für die Lagersensoren am Halterahmen wurden erneuert. Es wurde ein neuer Computer mit deutlich mehr Leistungs- 
potential installiert, so dass mehr Daten pro Zeiteinheit aufgenommen und auch gleichzeitig ausgewertet werden konnten. Die Mess- und Auswertungsprogramme wurden stets modifiziert, um einen reibungslosen Messablauf zu gewährleisten.

Es gibt viele interessante Fragen, die noch zu beantworten sind. Der Bedarf an biomechanischer Forschung gerade im Bereich der Wirbelsäule wird zukünftig noch stark ansteigen. 


\section{Zusammenfassung}

\subsection{Stand der Forschung}

Für das tiefere Verständnis von der Komplexität der Wirbelsäulenbewegung ist die Betrachtung der biomechanischen Eigenschaften der einzelnen Bewegungssegmente und deren funktionelles Zusammenwirken in einer physiologischen Bewegung unerlässlich.

Die Aufschlüsselung und Kenntnis der Wirbelsäulenkinematik ist nach heutigem Forschungsstand nur als mangelhaft zu beurteilen. Die wenigen Studien zur Mechanik der Brustwirbelsegmente und der Wirbelbogensegmente allgemein sind physikalisch nicht hinreichend, da zum Einen die verwendeten Messapparaturen nicht die nötige räumliche Auflösung und Präzision aufweisen und zum Anderen keine Erfassung aller 6 kinematische Freiheitsgraden erlauben.

\subsection{Experimentelle Methode}

In dieser in-vitro-Studie wird die Bewegungsstruktur zweier Brustwirbelsegmente (Th 2/3) beschrieben. Die Messung erfolgt mit Hilfe von 6 linearen Lagersensoren, die eine Erfassung der räumlichen Lageänderung des oberen Wirbelkörpers hochaufgelöst und in Abhängigkeit von zeitlich sich verändernden Drehmomenten erlauben. Die Wirkungslinien der Vorlasten werden in einem Kraftsystem geometrisch präzise eingestellt.

Die Messapparatur wurde weiterentwickelt und entsprechende Analyseverfahren verfeinert. Die differentielle Auflösung der Bewegungsstruktur beträgt für die Translation mindestens 2,5 $\mu \mathrm{m}$ und für die Rotation $0,03^{\circ}$. Diese beispiellose Messauflösung ermöglicht in der Auswertung eine Bestimmung der Position von momentanen Schraubachsen (IHA) im Raum bis auf ca. $1 \mathrm{~mm}$ genau. Damit kann postuliert werden, dass durch eine Erfassung von differentiell kleinen Positions- 
änderungen die tatsächliche Bewegungsstruktur von zwei Brustwirbelsegmenten registriert wird.

Eine Kraftschraube $(\underline{T}(t), \underline{F})$ wird erzeugt und ihre Parameter werden in physiologischen Grenzen variiert. Der Bewegungsrichtung entsprechend wird erst ein axiales Drehmoment $T_{Z}$, dann ein laterales Drehmoment $T_{Y}$ und zum Schluss ein sagittales Drehmoment $T_{x}$ aufgebracht. Die Hauptversuchsparameter dieser Studie sind die axialen Kräfte $\left(\mathrm{F}_{z}\right)$ mit ihren millimetergenau definierten Wirkungslinien. Die axiale Vorlast wird in der sagittalen und transversalen Richtung verschoben, so dass der obere Wirbelkörper in einer Flexions-/ Extensionslage oder nach lateral verkippt.

Die Datenauswertung erfolgt anhand der Position des Ortsvektors $\underline{r}_{j}(\alpha)$ und des Durchstoßpunktes der momentanen Schraubachse (IHA) durch eine Referenzebene (Rastpolkurve), ihre Ausrichtung im Raum anhand des Richtungsvektors $\underline{\mathrm{e}}(\alpha)$ sowie anhand der Schraubsteigung $\tau(\alpha)$, der Drehwinkel-DrehmomentKennlinie $\alpha(T)$ und der differentiellen Steifigkeit $d T / d \alpha$. Die Darstellung von wandernden momentanen Schraubachsen ermöglicht eine eindeutige Zuordnung der Bewegung eines Brustwirbelsegments zu den anatomischen Strukturen.

Es werden die Modelle der Getriebelehre und der Technischen Mechanik angewendet, um die Führung durch die Wirbelbogengelenke qualitativ beschreiben zu können.

Die sukzessive Resektion beider Artt. zygapophysiales bei systematischer Variation der Versuchsparameter verdeutlicht die biomechanischen Funktionen der Wirbelbogengelenke. 


\subsection{Ergebnisse}

\subsubsection{Axiale Rotation}

Im Experiment „Axiale Rotation“ zeigt sich bei beiden Bewegungssegmenten (Th 2/3 A und B) eine deutliche Unabhängigkeit der Bewegungsstruktur von der Position der axialen Vorlast. Die berechneten Schraubachsen sind im Drehwinkelbereich von $\alpha= \pm 1^{\circ}$ parallel zueinander und zum angreifenden Drehm oment $T_{z}$.

In Flexionslage bei ventralen Vorlasten kommt es in den Rotationsmaxima zu einer einseitigen Führung des Bewegungssegmentes durch das jeweilige Bogengelenk, so dass bei einer Rechtsdrehung die linke Art. zygapophysialis und umgekehrt bei der Linksdrehung das rechte Bogengelenk kraftschlüssig wird und in Funktion tritt.

Bei dorsalen Vorlasten, in Extensionslage, führen sofort beide Gelenke. Mit ansteigendem Drehwinkel a verändern sich nur die Bedingungen des Kraftschlusses in beiden Gelenken. Bei der Linksdrehung ( $\alpha>+19$ wird das rechte Gelenk zunehmend belastet und das linke Gelenk zunehmend entlastet, so dass schließlich nur noch das rechte Gelenk führt. Das entlastete Bogengelenk entkoppelt aber nicht vollständig, so dass eine ständige Führung beider Gelenke gegeben ist. Die Rotationssteifigkeit des Segments ist erhöht.

Im intakten Bewegungssegment ist die Gelenkführung so ausgelegt, dass bei einer axialen Rotationsbewegung im physiologischen Drehwinkelbereich und für alle Vorlastpositionen die Durchstoßpunkte der momentanen Schraubachsen stets in der Bandscheibe liegen.

Die sukzessive Resektion des rechten und dann des linken Bogengelenks beeinflusst die Lage der momentanen Schraubachse und das Steifigkeitsverhalten im Segment kaum.

Schlussfolgernd haben die Wirbelbogengelenke kaum Einfluss auf die Lage der momentanen Schraubachse. Auf eine Steifigkeitserhöhung bei einer unphysiologischen Belastung wird verzichtet, da eine ausreichende Stabilisierung des Segments durch die Brustkorbaufhängung gegeben ist. 


\subsubsection{Flexion und Extension}

Die Rastpolkurve verläuft knapp oberhalb der Bandscheibe des Th3/4-Segments. Die Wanderung der IHA in sagittaler und in vertikaler Richtung ist gering $(\Delta x=4 \mathrm{~mm}, \Delta \mathrm{z}=4 \mathrm{~mm})$, so dass in einem physiologischen Drehwinkelbereich $(\alpha= \pm 19$ die gemessenen momentanen Schraubachsen nahez $u$ ortsfest sind. Die Richtungsvektoren sind nahezu parallel zum angreifenden Drehmoment $T_{Y}$ ausgerichtet. Eine Verschiebung der axialen Vorlasten sowohl nach ventral/ dorsal als auch nach lateral verändert die Bewegungsparameter kaum.

Bei der Flexion entkoppeln die Wirbelbogengelenke. Bei der Extension sind die Wirbelbogengelenke kraftschlüssig. Der Extensionswinkel ist gering und beträgt für zentrale Vorlast nur $1^{\circ}$. Die minimale Steifigke it steigt in der Extension in Vergleich zur Flexion.

Bei Flexions-/ Extensionsbewegung zeigt sich eine leichte Asymmetrie der Drehwinkel-Drehmoment-Kennlinie. Bei extremen dorsalen/ventralen Vorlastpositionen weicht die Drehwinkel-Drehmoment-Kennlinie von ihrer sigmoiden Form ab und besitzt eher ein hockeyschlägerförmiges Aussehen.

Bei einer sukzessiven Resektion der Artt. zygapophysiales kommt es zu einer sukzessiven Wanderung der Rastpolkurve nach kranial.

Die Lage der momentanen Schraubachsen auf der Höhe des unmittelbar darunter liegenden Bewegungssegments führt zu der Annahme, dass die zwei benachbarten Bewegungssegmente Th2/3 und Th3/4 eine gemeinsame Flexionsachse besitzen und somit eine funktionelle Einheit bilden.

\subsubsection{Lateralflexion}

Analog der Flexions-/ Extensionsbewegung verläuft die Bahn der Rastpolkurve caudal. Bei einem absoluten Drehwinkel $\alpha= \pm 0,9^{\circ}$ liegen die Durchstoßpunkte der IHA mittig und knapp unterhalb der Bandscheibe Th3/4. Das applizierte Drehmoment $T_{X}$ führt zu einer beinahe reinen Seitwärtsneigung des Segments. Die 
Wanderung der IHA beträgt ca. $\pm 12 \mathrm{~mm}$, so dass neben der Bandscheibe und dem lateralen Bandapparat eine Beeinflussung durch die Wirbelbogengelenke gegeben ist.

Analog der Flexions-/ Extensionsbewegung bewirkt die sukzessive Resektion der Artt. zygapophysiales eine Verlagerung der Rastpolkurve nach kranial.

Die Bewegungsstruktur des Brustwirbelsegments Th2/3 bei der Lateralflexion von den Wirbelbogengelenken wird mit beeinflusst.

Analog der Flexion/ Extension wird auch bei der Lateralflexion eine gemeinsame Flexionsachse vermutet, welche die zwei benachbarten Bewegungssegmente Th2/3 und Th3/4 zu einer funktionellen Einheit verbindet.

\subsection{Diskussion}

Generell zeigt sich, dass die Wirbelbogengelenke die Lage der momentanen Schraubachsen kaum beeinflussen. Dennoch sind sie an der Modulation der Bewegungsstruktur mitbeteiligt.

Bei axialer Rotationsbewegung in physiologischen Bewegungsintervallen ist die Gelenkführung so ausgelegt, dass die Rastpolkurve stets im Zentrum der Bandscheibe zu liegen kommt. Die Lage der momentanen Schraubachse bleibt auch nach Resektion des rechten und dann des linken Bogengelenks nahezu unverändert. Der Verlauf der Rastpolkurven verändert sich, so dass nach der Resektion der rechten Art. zygapophysialis bei einer Linksdrehung die Bahn, die die Schraubachsen durchlaufen, einseitig verkürzt ist und nach beidseitigem Entfernen der Wirbelbogengelenke die momentanen Schraubachsen für den kompletten Drehwinkelbereich im Zentrum der Bandscheibe verbleiben. Schlussfolgernd führt die Entfernung der Wirbelbogengelenke zu quantitativen Änderungen der Bewegungsstruktur. Das Steifigkeitsverhalten im Bewegungssegment vor und nach der Resektion bleibt unverändert.

Die gemessenen Werte der Versuchsreihen Lateralflexion und Flexion/ Extension zeigen eine Wanderung der momentanen Schraubachsen mitten im Wirbelkörper des Th 3 und auf der Höhe der Artt. zygapophysiales des Bewegungssegmentes 
Th 3/4. Der Verlauf der Rastpolkurve führt zu der Annahme, dass die zwei benachbarten Bewegungssegmente Th2/3 und Th3/4 eine gemeinsame Flexionsachse besitzen und somit eine funktionelle Einheit bilden.

Die Junghannssche Bewegungssegmente sind als kleinste funktionelle Einheit der Wirbelsäule definiert. Die Erforschung ihrer biomechanischen Eigenschaften wird ein tieferes Verständnis von der Komplexität der kinematischen und statischen Eigenschaften der Wirbelsäule erlauben.

Die Weiterentwicklung der in dieser Studie vorgestellten Methodik würde die Effizienz der biomechanischen Forschung im Wirbelsäulenbereich massiv steigern. Man könnte Antworten auf viele interessante Fragen finden. So können in Zukunft die funktionellen Veränderungen nach Einsatz von Bandscheibenimplantaten in vitro getestet werden. 


\section{Abkürzungsverzeichnis}

\begin{tabular}{|l|l|}
\hline$\uparrow$ & Symbol für Zunahme/Verstärkung/ Steigerung \\
\hline$\downarrow$ & Symbol für Abnahme/Schwächung/ Reduktion \\
\hline 3D/6D & Drei-, sechsdimensional, räumlich \\
\hline Abb. & Abbildung \\
\hline ant. & anterior, vorne \\
\hline ap & anterior-posterior, Strahlenrichtung bei Projektion \\
\hline Art. & Articulatio \\
\hline Artt. & Articulationes (Plural) \\
\hline BWS & Brustwirbelsäule \\
\hline cm/ deg & Zentimeter pro Grad, Einheit des Versatzes \\
\hline cos & Kosinus \\
\hline CT & Computertomographie \\
\hline D & Steifigkeitssymbol \\
\hline Fix. & Fixiert, fest \\
\hline HWS & Halswirbelsäule \\
\hline K & Kontaktpunkt \\
\hline Ko-x & Koordinatensystem 0-x \\
\hline LG & Gestell \\
\hline li. & links \\
\hline Lig. & Ligamentum, Band \\
\hline Ligg. & Ligamenta, Bänder \\
\hline LK & Koppel \\
\hline LWS & Lendenwirbelsäule \\
\hline M & Mittelpunkt \\
\hline M. & Musculus \\
\hline Mm. & Musculi \\
\hline N & Newton, Einheit der Kraft \\
\hline Ncm & Newtonzentimeter, Einheit der Energie \\
\hline & \\
\hline
\end{tabular}




\begin{tabular}{|l|l|}
\hline Ncm/deg & Newtonzentimeter pro Grad, Einheit der Steifigkeit \\
\hline neg. & negativ \\
\hline P & Drehpol, Ort der momentanen Drehachse \\
\hline pos. & Positiv \\
\hline post. & posterior, hinten \\
\hline Proc. & Processus, Fortsatz \\
\hline Procc. & Processus, Fortsätze (Plural) \\
\hline R & \multicolumn{1}{c|}{\begin{tabular}{c|} 
2. Pleuel Durchstoßpunkte der momentanen Schraubachse durch \\
eine Ebene
\end{tabular}} \\
\hline re. & Rechts \\
\hline Res. & Resektion \\
\hline ROM & Range of motion, Bewegungsumfang \\
\hline sin & Sinus \\
\hline T $1-6$ & Messtaster (1 bis 6) \\
\hline Th2/3 & Segmenthöhe der Brustwirbelsäule, Wirbel 2-3 \\
\hline tan & Tangens \\
\hline VL & Vorlast \\
\hline W & Widerstandszentrum \\
\hline WBG & Wirbelbogengelenke \\
\hline WZ & Widerstandszentrum \\
\hline
\end{tabular}




\section{Literaturverzeichnis}

Abumi K, Panjabi MM, Kramer K, Durancem J, Oxland T, Crisco J (1990): Biomechanical evaluation of lumbar spinal stability after graded facetectomies. Spine 15-11, $1142-1147$

Ahmed AM, Duncan NA, Burg DL (1990): The effect of facetgeometrie on the axial torque rotation response of the lumbar motion segments. Spine $\underline{15}, 391-$ 401

Benninghoff A, Drenkhahn D: Makroskopische Anatomie, Embryologie und Histologie des Menschen. Bd. 1, Urban\&Fischer, München 2003

BLÜMLE A: Beitrag zur funktionellen Anatomie und der neuromuskulären Steuerung des Kniegelenks am Modelltier Schwein. Med. Diss. Greifswald 1997

BockermanN V, Abteilung Neurochirurgie der Universität Göttingen: Mündliche Mitteilung. 2001

Bockermann V: Kinematik des Halswirbelsäulen-Segments C5/6. Biomechanische Analyse seines Bewegungsmusters bei physiologischen Standardbelastungen. Med. Diss. Greifswald 2004

Bogduk N, Twomey: Clinical anatomy of the lumbar spine. Churchill Livingstone, London/ New York/ Tokio 1991

ČıнÁK R: Die Morphologie und Entwicklung der Wirbelbogengelenke. Die Wirbelsäule in Forschung und Praxis. Bd. 87; Hippokrates-Verlag, Stuttgart 1981

Dathe H: Der elastisch aufgehängte Körper. Physikalische Diss. Göttingen 2001

El-Bohy AA, Yang K-H, KING Al (1989): Experimental verification of facet load transmission by direct measurements of facet laminar contact pressure. $J$ Biomech $\underline{22-8}, 931-941$

FANGHÄNEL J, SchULz F (1962): Mitteilung über eine Konservierungsflüssigkeit für anatomisches Präpariermaterial. Z Med Labortech $\underline{3}, 329$ - 332

FARFAN HF: Biomechanik der Wirbelsäule. Hippokrates-Verlag, Stuttgart 1973

FARFAN HF: Biomechanik der Lendenwirbelsäule. Die Wirbelsäule in Forschung und Praxis. Bd. 80; Hippokrates-Verlag, Stuttgart 1979

Fıck R: Handbuch der Anatomie und Mechanik der Gelenke unter Berücksichtigung der bewegenden Muskeln. Bd.1; Anatomie der Gelenke. G.Fischer Jena $1910 \mathrm{a}$ 
FIck R: Handbuch der Anatomie und Mechanik der Gelenke unter Berücksichtigung der bewegenden Muskeln. Allgemeine Gelenk- und Muskelmechanik. Bd.2; G.Fischer, Jena 1910b

FIck R: Handbuch der Anatomie und Mechanik der Gelenke unter Berücksichtigung der bewegenden Muskeln. Spezielle Gelenk- und Muskelmechanik. Bd.3; G.Fischer, Jena 1911

Frick R, Leonhardt H, Starck D: Allgemeine Anatomie. Spezielle Anatomie I. Bd.1; Thieme, Stuttgart 1992

Goel VK, Clark CR, Harris KG, Schulte KR (1988): Kinematics of the cervical spine: Effects of multiple total laminectomy and facet wiring. J Orthop Res $\underline{6}$, $611-619$

Götz W, Krengel S, Quondamatteo F, Miosge N, Herken R: Cell Biological Aspects of Early Human Vertebral Column Development. In: Vogel R, Fanghänel J, Giebel J (Hrsg.): Aspects of Teratology. Tectum Verlag Marburg 1996, 81-89

Götz W, Bertagnoli R, Herken R: Struktur und Zusammensetzung der extrazellulären Matrix normaler menschlicher Nuclei pulposi. In: Wilke H-J, Glaes L E (Hrsg): Die traumatische und degenerative Bandscheibe. (Hefte zu „Unfallchirurgie“, Bd. 271), Springer, Heidelberg 1999, 3-12

GoldsteIn H: Klassische Mechanik. 7. Auflage Akademische Verlagsgesellschaft, Wiesbaden 1983

Goodwin RR, James KS, Daniels AU, Dunn HK (1994): Distraction and compression loads enhance spine torsional stiffness. J Biomech 27, 1049-1057

Gregersen GG, Lucas DB (1967): An In Vivo Study of the Axial Rotation of the Human Thoracolumbar Spine. J Bone Joint Surg. 49, 247-262

JunghanNs H: Nomenclatura columnae vertebralis. (Die Wirbelsäule in Forschung und Praxis. Bd. 75); Hippokrates-Verlag, Stuttgart 1977

KaLSCHEUER A: Kinematik des Halswirbelsäulensegments C3/C4 unter systematischer Vorlastvariation. Med. Diss. Göttingen 2001

KAPANDJI IA: Funktionelle Anatomie der Gelenke. Rumpf und Wirbelsäule. Bd. III; Enke Verlag, Stuttgart 1985

Kohlmann T, Deck R, Klockgether R, Raspe H, Brockow T, Engster M (1998): Rückenschmerzen in der Lübecker Bevölkerung: Syndrome, Krankheitsverhalten und Versorgung. Z Rheumatol $\underline{57}, 238-240$

KräMER J: Bandscheibenbedingte Erkrankungen. 2.Auflage; Thieme, Stuttgart 1987

KrämeR J, GrifKa J: Orthopädie. 7.Auflage; Springer, Heidelberg 2005

Krämer J, GrifKA J: Orthopädie. Unfallchirurgie. 8.Auflage; Springer, Heidelberg 2007 
KRISMER M : Die Rotation der Brust- und Lendenwirbelsäule. (Aktuelle Probleme in Chirurgie und Orthopädie. Bd. 43); Verlag Hans Huber, Bern/Göttingen/Toronto/ Seattle1996

Kubein-Meesenburg D, NäGerL H, Fanghänel J (1990): Elements of a general theory of joints. 1. Basic kinematic and static function of diarthrosis. Anat Anz $170,301-308$

Kubein-Meesenburg D, NäGerl H, Fanghänel J (1991a): Elements of a general theory of joints. 4. Coupled joints as a simple gear system. Anat Anz 172, $309-$ 321

Kubein-Meesenburg D, NäGerl H, Fanghänel J (1991b): Elements of a general theory of joints. 5. Basic mechanism of the knee. Anat Anz 173, 131-142

Kubein-Meesenburg D, Nägerl H, Cotta H, Fanghänel J (1993): Biomechanische Prinzipien in Diarthrosen und Synarthrosen. Teil I: Grundbegriffe bei Diarthrosen. Z Orthop 131, 97 - 104

LEE D: Zur räumlichen Kinematik der Wirbelsäule. Eine mathematischphysikalische und experimentelle Untersuchung des Brustwirbelsäulensegments Th 6/7. Med. Diss. Göttingen 2003

LIN HS, LIU YK, Adams KH (1978): Mechanical Response of the Lumbar intervertabral joint under physiological (complex) loading. J Bone Joint Surg., 60-A, $41-55$

LIPPERT H: Lehrbuch der Anatomie. Elsevier. München 2003

LuschKa H: Die Nerven des menschlichen Wirbelkanals. H. Laub, Tübingen 1856

LUTZ G (1967): Die Entwicklung der kleinen Wirbelgelenke. Z Orthop., 104, 19-28

MACConalLL MA (1953): The movements of bones and joints. 5. The significance of shape. J Bone Joint Surg 35 B, $290-297$

MANSOUR M: Die biomechanische Funktion der Articulationes zygappophysiales der Lendenwirbelsäule - eine 6-D Analyse der Bewegungsstrukturen des Segments L 3/4. Med. Diss. Göttingen 2001

mansour M, Spiering S, Lee C, Dathe h, Karlscheuer A, Kubein-Meesenburg D, NÄGERL H (2004): Evidence for IHA Migration during axial rotation of a lumbar spine segment by using a novel High-resolution 6D Kinematic Tracking System. J Biomech., $\underline{37}, 583-592$

McLAIN RF (1994): Mechanoreceptor endings in human cervical facet joints. Spine $19,495-501$

MilLeR JA, SKogland LB: On the load-displacement behaviour of adolescent spinal motion segments - an experimental study using autopsy specimens. Ph.D. Thesis Oslo 1980 
MyeRs BS, McElhancy JH, BRIAN JD (1991): The viscoelastic responses of the human cervical spine in torsion : Experimental limitations of quasi-linear theory, and method for reducing these effects. J Biomech 24-9, 811-817

NÄGERL H : Biomechanische Prinzipien in Diarthrosen und Synarthrosen. Med. Habil.-Schr. Göttingen 1990

NäGerl H, Kubein-Meesenburg D, Fanghänel J (1990): Elements of a general theory of joints. 2. Introduction into a theory of synarthrosis. Ann Anat 171, 323 $-333$

NäGerL H, Kubein-Meesenburg D, Fanghänel J (1992): Elements of a general theory of joints. 7. Mechanical structures of the relative motion of adjacent vertebrae. Ann Anat 174, 66 - 75

Nägerl H, Kubein-Meesenburg D, Cotta H, Fanghänel J, Kirsch S (1993a): Biomechanische Prinzipien in Diarthrosen und Synarthrosen. Teil II: Die Articulatio Humeri als Dimeres Kugelgelenk. Z Orthop 131, 293 - 301

Nägerl H, Kubein-Meesenburg D, Cotta H, Fanghänel J, Kirsch S (1993b): Biomechanische Prinzipien in Diarthrosen und Synarthrosen. Teil III: Die Mechanik des Tibiofemoralgelenks und die Rolle der Kreuzbänder. Z Orthop 131, $385-396$

Nägerl H, Kubein-Meesenburg D, Cotta H, fanghänel J, Rossow A, Spiering S (1995): Biomechanische Prinzipien in Diarthrosen und Synarthrosen. Teil IV: Zur Biomechanik der Wirbelsäule im Lendenbereich. Eine Pilotstudie. Z Orthop $\underline{133}, 481-491$

NetTer FH: Atlas der Anatomie des Menschen. 3. Aufl., Thieme Verlag, Stuttgart 2003

Niethard FU, Pfeil J: Orthopädie. Hippokrates-Verlag, Stuttgart 1989

Panjabi MM, White AA, BRAND RA (1974): A note on defining body parts configurations. J Biomech $\underline{7}, 385-387$

Panjabi MM, Brand RM, White AA (1976): Three dimensional flexibility and stifness of human thoracic spine. J Biomech $\underline{9}, 185-192$

Panjabi MM, Krag MH, Goel VK (1981): A technique for measurement and descripton of three-dimensional six degree-of-freedom motion of a body joint with an application to the human spine. J Biomech 14, $447-460$

Panjabi Mm, Summers DJ, Pelker RR, Videman T, Friedlaender Ge, Southwick WO (1986): Three-dimensional load-displacement curves due to forces on the cervical spine. J Orthop Res 4, $152-161$

Panjabi mM, Goel V, Oxland Z, Takata K, Duranceau J, Krag M, Price M (1991): Human lumbar vertebra: Quantitative three-dimensional anatomy. Spine 17, $299-306$ 
Parnianpour M, Li F, Nordin M, Kahanovitz N (1989): A data base of isointerial trunc strength test against three resistance levels in sagittal frontal and transverse plancs in normal male subjects. Spine 14, $409-411$

Patwardhan AG, Havey RM, Garandang G, Siomonds J, Voronov LI, Ghanayem AJ, MeADE KP, Gavin TM, Paxinos O (2002): Effect of compressive follower preload on the flexion-extension response of the human lumbar spine. $J$ Orthop Res $\underline{21}, 540-546$

PuTz R: Funktionelle Anatomie der Wirbelgelenke. (Normale und pathologische Anatomie. Bd. 43); Thieme, Stuttgart 1981

RALSTON HJ (1961): Uses and limitations of electromyography in quantitative study of skeletal muscle function. Am J Orthod Dentofac 47, 521 - 526

Raspe H, Kohlmann T: Die aktuelle Rückenschmerz-Epidemie. In: Pfingsten M.,Hildebrandt J.: Chronische Rückenschmerzen. Wege aus dem Dilemma, Hans Huber, Bern 1998, 20-23

RASPE H (2001): Back pain. In: Schauder P., Berthold H., Eckel H., Ollenschläger G. (Hrsg): Zukunft sichern: Senkung der Zahl chronisch Kranker. Verwirklichung einer realistischen Utopie. Deutscher Ärzteverlag, Köln 2006

Rauber A, Kopsch F: Anatomie des Menschen. Lehrbuch und Atlas. Bd. I: Bewegungsapparat; hrsg. u. bearb. v. TilLmanN B, TönduRY G; Thieme, Stuttgart 1987

SCHÄFER W: Messung der räumlichen Mikrobewegung und der Verbiegung des Femurschaftes von Hüft-Endo-Prothesen in Abhängigkeit eines räumlichen Kraftsystems. Phys. Diplomarbeit (IV. Physikalisches Institut der Georg-AugustUniversität Göttingen) Göttingen 1995

SchiebleR TH, Korf HW: Anatomie. Steinkopff Verlag. Heidelberg 2007

Schildhauer T, Robie BH, Klein R, O'Leary P: The effect of joint compressive load on the motion of the human lumbo-sacral spine. In: Proceeding of the 40th. Orthopaedic Research Society, 0.0.1994

ScHÜNKE M: Funktionelle Anatomie. Topographie und Funktion des Bewegungssystems. Thieme Verlag. Stuttgart 2000

Soвbota. Putz R, Kopsch F: Sobotta. Allgemeine Anatomie. Bewegungsapparat. Innere Organe. Neuroanatomie. 22. Aufl., Elsevier. München 2007

SPIERING S: Kinematik der Lendenwirbelsäule unter Lastvariationen. Phys. Diplomarbeit (IV. Physikalisches Institut der Georg-August-Universität Göttingen) Göttingen 1995

SPIERING S: Drei Methoden zur Evaluation der mechanischen Funktion von Synarthrosen und Diarthrosen. Med. Diss. Greifswald 2002 
Teichmann H: Physikalische Anwendungen der Vektor- und Tensorrechnung. Bd. 1; BI-Verlag, Mannheim/Wien/Zürich 1973

TönDuRY G: Entwicklungsgeschichte und Fehlbildungen der Wirbelsäule. Hippokrates-Verlag, Stuttgart 1958

WACHOWSKI M: Biomechanik des C3/C4-Segments; Bedeutung der Unkovertebralund Wirbelbogengelenke für die Kinematik und Steifigkeit. Med. Diss. Göttingen 2005

Wachowski mm, Mansour M, Hawallek T, Kubein-Meesenburg D, Huber J, NÄGERL H (2009a): Parametric Control of the Stiffness of Lumbar Segments Strain (2009) doi:10.1111/j. 1475-1305.2009.00686.x IF: 1.154

Wachowski MM, Mansour M, Lee C, Ackenhausen A, Spiering S, Fanghänel J, DUMONT C, Kubein-MEesenbuRG D, NÄGERL H (2009b): How do spinal segments move? J Biomech. 2009 Aug 12. [Epub ahead of print] IF: 2.784

Waldeyer A, Mayet A: Anatomie des Menschen. Fanghänel J, Pera F, Anderbuher F, Nitsch R (Hrsg.), Bd.1, Walter de Gruyter, Berlin/New York 2002

WALMSLEY T (1928): The articular mechanism of the diarthroses. J Bone Joint Surg $\underline{10}, 40-48$

WASSILEV W, KüHNEL W (1992): Struktur und Funktion der Zwischenwirbelscheibe. Ann. Anat. 174, $54-65$

WILKE HJ: Experimentelle Untersuchung zur Biomechanik der Wirbelsäule und ihrer Stabilisierung. Humanbiologische Diss. Ulm 1993

WiLKE HJ, KrISchaK S, LUTZ EC (1996): Formalin fixation strongly influences biomechanical properties of the spine. J Biomech. 29, $1629-1631$

Wilke HJ, Kettler A, Hartwig E, Schulteheib M, Claes L (2002): Mechanically simulated muscle forces strongly stabilize intact and upper cervical spine specimens. J Biomech. 35, $339-346$

WoLF K: Lehrbuch der Technischen Mechanik starrer Systeme. Verlag Julius Springer, Wien 1931

Zukschwerdt L, Emminger E, Biedermann F, Zettel H: Wirbelgelenk und Bandscheibe. Hippokrates Verlag, Stuttgart 1960 


\section{Danksagung}

Die vorliegende Arbeit ist in der interdisziplinären Arbeitsgruppe Biomechanik Göttingen-Greifswald entstanden. Beteiligt waren u. a. Prof. Dr. rer. Nat. H. Nägerl vom IV. Physikalischen Institut, sowie Prof. Dr. med. dent. D. Kubein-Meesenburg aus der Abteilung Kieferorthopädie im Zentrum Zahn-, Mund- und Kieferheilkunde der Georg-August-Universität Göttingen.

Herrn Prof. Dr. H. Nägerl möchte ich für seine Unterstützung bei dieser Arbeit und für seine hervorragende wissenschaftliche Anleitung danken.

Mein Dank gilt Herrn Prof. Dr. D. Kubein-Meesenburg für seine Förderung meiner wissenschaftlichen Arbeit.

Mein besonderer Dank gilt Herrn Dr. Wachowski, der mir immer helfend zur Seite stand, denn ohne seine Unterstützung wäre diese Arbeit in dieser Form nicht möglich gewesen.

Weiterhin möchte ich den Mitgliedern der Arbeitsgruppe Biomechanik für die gute und anregende Zusammenarbeit danken. Für den Bau und die Weiterentwicklung der Messapparatur möchte ich mich bei den Mitarbeitern der FeinmechanikWerkstatt für ihre Hilfe und bei Herrn Dipl. phys. S. Spiering bedanken.

Außerdem danke ich Frau Dr. S. Fricke-Zech, Frau Dr. J. Fialka-Fricke und Frau Dr. C. Hansen für die fortwährende tatkräftige Unterstützung und zahlreiche Anregungen bei der Textkorrektur dieser Arbeit. 


\section{Lebenslauf}

Am 26.09.1974 wurde ich in Aktjubinsk, Kasachstan, geboren. Von 1981-1991 besuchte ich die Mittelschule in Aktjubinsk und beendete dort meine Schulausbildung 1991 mit Erlangung der Hochschulreife. Von 1991 bis 1993 besuchte ich die Medizinische Fachschule in Aktjubinsk, die ich als Dentistin abgeschlossen habe. Nach meiner Übersiedlung in die BRD wurde diese Ausbildung 1993 dem Berufsabschluss einer Zahnarzthelferin gleichgestellt. In der Zeit von 1994 bis 1996 habe ich diesen Beruf erfolgreich in der Kieferorthopädischen Praxis Dr. J. Raiman in Hannover ausgeübt. Meine Berufstätigkeit habe ich im November 1996 unterbrochen um einen Lehrgang zur Erlangung der Allgemeinen Hochschulreife in Göttingen zu absolvieren. Diesen habe ich 1998 abgeschlossen und mich für das Studium der Zahnmedizin an der Georg-August-Universität Göttingen entschieden. Im März 2000 legte ich die Naturwissenschaftliche Vorprüfung ab. 2001 absolvierte ich dann die Zahnärztliche Vorprüfung und 2004 die Zahnärztliche Prüfung.

Im Rahmen meiner Weiterbildung zur Fachzahnärztin für Kieferorthopädie war ich in der Praxis Dres. Spohr und Schmidt in Kassel tätig. Im Anschluss an das Allgemein-Zahnärztliche Vorbereitungsjahr setzte ich hier meine Kieferorthopädische Weiterbildung fort. Im Januar 2007 kam mein Sohn Lev zur Welt. Nach meinem Erziehungsurlaub habe ich 2008 mit Freude die Möglichkeit der Weiterführung meiner Weiterbildung im Klinikum der Georg-August-Universität Göttingen in Anspruch genommen. Zurzeit bin ich in der Abteilung Kieferorthopädie im Zentrum Zahn-, Mund- und Kieferheilkunde tätig. Voraussichtlich im April 2010 werde ich meine fachärztliche Weiterbildung mit der Fachzahnarztprüfung abschließen. 